\title{
A dichotomy theorem for nonuniform CSPs
}

\author{
Andrei A. Bulatov
}

\begin{abstract}
In this paper we prove the Dichotomy Conjecture on the complexity of nonuniform constraint satisfaction problems posed by Feder and Vardi]
\end{abstract}

\section{Contents}

1 Introduction 3

I Outline of the algorithm 5

2 Introduction to CSP 5

2.1 CSP, universal algebra and the Dichotomy conjecture . . . . . . 5

2.2 Bounded width and the few subpowers algorithm . . . . . . 8

3 Solving CSPs $\quad 10$

3.1 Congruence separation and centralizers . . . . . . . . . 10

3.2 The algorithm . . . . . . . . . . . . . . . 12

II Technicalities 17

\footnotetext{
${ }^{1}$ Apart from correcting numerous typos and inaccuracies, this version of the paper is different from the first Arxiv version in the following ways:

- A self-contained high level presentation of the main results is added.

- Preliminaries section is extended.

- An inconsistency between the definition and the use of the chaining condition is fixed.

- The proof of Lemma 46 is expanded for improved readability. Also a gap in this proof is patched.

- Section "Auxiliary Lemmas" is split into two sections, Sections 7 and 8 and moved forward.

- The proof of Theorem 68 is reorganized and expanded for improved readability.
} 
4 Preliminaries 19

4.1 Universal algebra and CSP: notation and agreements . . . . . . 19

4.2 Minimal sets and polynomials . . . . . . . . . . . . . 21

4.3 Coloured graphs . . . . . . . . . . . . . . . . 23

4.4 Maximality ......................... 27

4.5 Quasi-decomposition and quasi-majority . . . . . . . . . . 30

4.6 Rectangularity . . . . . . . . . . . . . . . . . 31

5 Separating congruences 33

5.1 Special polynomials, mapping pairs . . . . . . . . . 33

5.2 Separation . . . . . . . . . . . . . . . . 35

5.3 Collapsing polynomials . . . . . . . . . . . . . . . . 43

5.4 Separation and minimal sets . . . . . . . . . . . 44

6 Centralizers and decomposition of CSPs 46

6.1 Quasi-Centralizer ... . . . . . . . . . . . . . 46

6.2 Decomposition of CSPs . . . . . . . . . . . . 50

7 The Congruence Lemma

8 Chaining $\quad 57$

9 Strategies and solutions $\quad 61$

9.1 The grand scheme . . . . . . . . . . . . . . . . . 61

9.2 Proof of Theorem $69 \ldots \ldots \ldots \ldots \ldots$

9.3 Strategies ....................... 67

10 Proof of Theorem 68

10.1 Tightening affine factors . . . . . . . . . . . . . 69

10.1.1 Transformation of the strategy and induced congruences . 69

10.1.2 Condition (S6) . . . . . . . . . . . . . . 72

10.1 .3 Condition $(\mathrm{S} 1) \ldots \ldots \ldots \ldots \ldots$

10.1.4 Conditions $(\mathrm{S} 2),(\mathrm{S} 3) \ldots \ldots \ldots \ldots \ldots$

10.1.5 The remaining conditions . . . . . . . . . 83

10.2 Tightening non-affine factors $\ldots \ldots \ldots \ldots$

10.2.1 Step $1 . \ldots \ldots \ldots \ldots \ldots$. . . . . . . . . . . . . . . . . . . . . . . .

10.2 .2 Step $2 . \ldots \ldots \ldots \ldots$ 


\section{Introduction}

In a Constraint Satisfaction Problem (CSP) the question is to decide whether or not it is possible to satisfy a given set of constraints. Constraints are often represented by specifying a relation, which is a set of allowed combinations of values some variables can take simultaneously. If the constraints allowed in a problem have to come from some set $\Gamma$ of relations, such a restricted problem is referred to as a nonuniform CSP and denoted $\operatorname{CSP}(\Gamma)$. The set $\Gamma$ is then called a constraint language. Nonuniform CSPs not only provide a powerful framework ubiquitous across a wide range of disciplines from theoretical computer science to computer vision, but also admit natural and elegant reformulations such as the homomorphism problem and a characterization as the class of problems equivalent to a logic class MMSNP. Many different versions of the CSP have been studied across various fields. These include CSPs over infinite sets, counting CSPs (and related Holant problem and the problem of computing partition functions), several variants of optimization CSPs, valued CSPs, quantified CSPs, and numerous related problems. The reader is referred to the recent book [48] for a survey of the stateof-the art in some of these areas. In this paper we, however, focus on the decision nonuniform CSP and its complexity.

A systematic study of the complexity of nonuniform CSPs was started by Schaefer in 1978 [57] who showed that for every constraint language $\Gamma$ over a 2-element set the problem $\operatorname{CSP}(\Gamma)$ is either solvable in polynomial time or is NPcomplete. Schaefer also asked about the complexity of $\operatorname{CSP}(\Gamma)$ for languages over larger sets. The next step in the study of nonuniform CSPs was made in the seminal paper by Feder and Vardi [33, 34], who apart from considering numerous aspects of the problem, posed the Dichotomy Conjecture that states that for every finite constraint language $\Gamma$ over a finite set the problem $\operatorname{CSP}(\Gamma)$ is either solvable in polynomial time or is NP-complete. This conjecture has become a focal point of the CSP research and most of the effort in this area revolves to some extent around the Dichotomy Conjecture.

The complexity of the CSP in general and the Dichotomy Conjecture in particular has been studied by several research communities using a variety of methods, each contributing an important aspect of the problem. The CSP has been an established area in artificial intelligence for decades, and apart from developing efficient general methods of solving CSPs researchers tried to identify tractable fragments of the problem [32]. The very important special case of the CSP, the (Di)Graph Homomorphism problem and the $H$-Coloring problem have been actively studied in the graph theory community, see, e.g. [37, 38] and subsequent works by Hell, Feder, Bang-Jensen, Rafiey and others. Homomorphism duality introduced in these works has been very useful in understanding the structure of constraint problems. 
The CSP plays a major role and has been successfully studied in database theory, logic and model theory [46, 45, 36], although the version of the problem mostly used there is not necessarily nonuniform. Logic games and strategies are a standard tool in most of CSP algorithms. An interesting approach to the Dichotomy Conjecture through long codes was suggested by Kun and Szegedy [49]. BrownCohen and Raghavendra proposed to study the conjecture using techniques based on decay of correlations [12]. In this paper we use the algebraic structure of the CSP, which is briefly discussed next.

The most effective approach to the study of the CSP turned out to be the algebraic approach that associates every constraint language with its (universal) algebra of polymorphisms. The method was first developed in a series of papers by Jeavons and coauthors [41, 42, 43] and then refined by Bulatov, Krokhin, Barto, Kozik, Maroti, Zhuk and others [5, 8, 6, 3, 26, 16, 28, 52, 53, 58, 60, 59]. While the complexity of $\operatorname{CSP}(\Gamma)$ has been already solved for some interesting classes of structures such as graphs [37], the algebraic approach allowed the researchers to confirm the Dichotomy Conjecture in a number of more general cases: for languages over a set of size up to 7 [14, 17, 51, 60, 59], so called conservative languages [15, 18, 19, 2], and some classes of digraphs [7]. It also allowed to design the main classes of CSP algorithms [6, 24, 21, 11, 40], and refine the exact complexity of the CSP [1, 8, 31, 50].

In this paper we confirm the Dichotomy Conjecture for arbitrary languages over finite sets. More precisely we prove the following

Theorem 1 For any finite constraint language $\Gamma$ over a finite set the problem $\mathrm{CSP}(\Gamma)$ is either solvable in polynomial time or is NP-complete.

The proved criterion matches the algebraic form of the Dichotomy Conjecture suggested in [26]. The hardness part of the conjecture has been known for long time. Therefore the main achievement of this paper is a polynomial time algorithm for problems satisfying the tractability condition. More specifically, we suggest such an algorithm for languages that contain all the constant relations of the form $\{(a)\}$, and this implies a general dichotomy due to the results of [26].

Using the algebraic language we can state the result in a stronger form. Let $\mathbb{A}$ be a finite idempotent algebra and let $\operatorname{CSP}(\mathbb{A})$ denote the union of problems $\operatorname{CSP}(\Gamma)$ such that every term operation of $\mathbb{A}$ is a polymorphism of $\Gamma$. Problem $\operatorname{CSP}(\mathbb{A})$ is no longer a nonuniform CSP, and Theorem 1 allows for problems $\operatorname{CSP}(\Gamma) \subseteq \operatorname{CSP}(\mathbb{A})$ to have different solution algorithms even when $\mathbb{A}$ meets the tractability condition. We show that the solution algorithm only depends on the algebra $\mathbb{A}$. 
Theorem 2 For a finite idempotent algebra that satisfies the conditions of the Dichotomy Conjecture there is a uniform solution algorithm for $\operatorname{CSP}(\mathbb{A})$.

An interesting question arising from Theorems 112 is known as the Metaproblem: Given a constraint language or a finite algebra, decide whether or not it satisfies the conditions of the theorems. The answer to this question is not quite trivial, for a thorough study of the Meta-problem see [30, 35].

The paper consists of two parts. The first part of the paper aims at a selfcontained introduction into the main ideas of the solution algorithm. The second part mostly concerns with further development of the algebraic approach and technical proofs of the results, and is significantly more involved. We start with introducing the terminology and notation for CSPs that is used throughout the paper and reminding the basics of the algebraic approach. Then in Section 3.1 we introduce the key ingredients used in the algorithm: separating congruences and quasi-centralizer. Then in Section 3.2 we apply these concepts to CSPs, first, to demonstrate how quasi-centralizers help to decompose an instance into smaller subinstances, and, second, to introduce a new kind of minimality condition for CSPs, block minimality. After that we state the main results used by the algorithm and describe the algorithm itself. We complete the first part by introducing the main technical construction to give an idea of why the algorithm works.

\section{Part I}

\section{Outline of the algorithm}

\section{Introduction to CSP}

For a detailed introduction to CSP and the algebraic approach to its structure the reader is referred to a very recent and very nice survey by Barto et al. [9]. Basics of universal algebra can be learned from the textbook [29]. In preliminaries to this paper we therefore focus on what is needed for our result.

\subsection{CSP, universal algebra and the Dichotomy conjecture}

The 'combinatorial' formulation of the CSP best fits our purpose. Fix a finite set $A$ and let $\Gamma$ be a constraint language over $A$, that is, a set - not necessarily finite - of relations over A. The (nonuniform) Constraint Satisfaction Problem (CSP) associated with language $\Gamma$ is the problem $\operatorname{CSP}(\Gamma)$, in which, an instance is a pair $(V, \mathcal{C})$, where $V$ is a set of variables; and $\mathcal{C}$ is a set of constraints, i.e. pairs $\langle\mathbf{s}, R\rangle$, 
where $\mathbf{s}=\left(v_{1}, \ldots, v_{k}\right)$ is a tuple of variables from $V$, the constraint scope, and $R \in \Gamma$, the $k$-ary constraint relation. We always assume that relations are given explicitly by a list of tuples. The way constraints are represented does not matter if $\Gamma$ is finite, of course, but it may change the complexity of the problems for infinite languages. The goal is to find a solution, that is a mapping $\varphi: V \rightarrow A$ such that for every constraint $\langle\mathbf{s}, R\rangle, \varphi(\mathbf{s}) \in R$.

We will often use the set of solutions of a CSP instance $\mathcal{P}=(V, \mathcal{C})$ or its subproblems (to be defined later), viewed either as a $|V|$-ary relation or as a set of mappings $\varphi: V \rightarrow A$. It will be denoted by $\mathcal{S}_{\mathcal{P}}$, or just $\mathcal{S}$ if $\mathcal{P}$ is clear from the context.

Jeavons et al. in [41, 42] were the first to observe that higher order symmetries of constraint languages called polymorphisms play a significant role in the study of the complexity of the CSP. A polymorphism of a relation $R$ over $A$ is an operation $f\left(x_{1}, \ldots, x_{k}\right)$ on $A$ such that for any choice of $\mathbf{a}_{1}, \ldots, \mathbf{a}_{k} \in R$ we have $f\left(\mathbf{a}_{1}, \ldots, \mathbf{a}_{k}\right) \in R$. If this is the case we also say that $f$ preserves $R$, or that $R$ is invariant with respect to $f$. A polymorphism of a constraint language $\Gamma$ is an operation that is a polymorphism of every $R \in \Gamma$.

Theorem $3([41,42])$ For constraint languages $\Gamma, \Delta$, where $\Gamma$ is finite, if every polymorphism of $\Delta$ is also a polymorphism of $\Gamma$, then $\operatorname{CSP}(\Gamma)$ is polynomial time reducible to $\operatorname{CSP}(\Delta)$. (In fact, this can be improved to a log-space reduction.)

Listed below are the several types of polymorphisms that occur frequently throughout the paper. The presence of each of these polymorphisms imposes restrictions on the structure of invariant relations that can be used in designing a solution algorithm. Some of such results we will mention later.

- Semilattice operation is a binary operation $f(x, y)$ such that $f(x, x)=x, f(x, y)=$ $f(y, x)$, and $f(x, f(y, z))=f(f(x, y), z)$ for all $x, y, z \in A$;

- $k$-ary near-unanimity operation is a $k$-ary operation $u\left(x_{1}, \ldots, x_{k}\right)$ such that $u(y, x, \ldots, x)=u(x, y, x, \ldots, x)=\cdots=u(x, \ldots, x, y)=x$ for all $x, y \in A ;$ a ternary near-unanimity operation $m$ is said to be a majority operation, it satisfies the equations $m(y, x, x)=m(x, y, x)=m(x, x, y)=x$;

- Mal'tsev operation is a ternary operation $h(x, y, z)$ satisfying the equations $h(x, y, y)=$ $h(y, y, x)=x$ for all $x, y \in A$; the affine operation $x-y+z$ of an Abelian group is a special case of Mal'tsev operations;

- $k$-ary weak near-unanimity operation is a $k$-ary operation $w$ that satisfies the same equations as a near-unanimity operations $w(y, x, \ldots, x)=w(x, y, x, \ldots, x)=$ $\cdots=w(x, \ldots, x, y)$, except for the last one.

The next step in discovering more structure behind nonuniform CSPs has been 
made in [26], in which universal algebras were brought into the picture. A (universal) algebra is a pair $\mathbb{A}=(A, F)$ consisting of a set $A$, the universe of $\mathbb{A}$, and a set $F$ of operations on $A$. Operations from $F$ together with operations that can be obtained from them by means of composition are called the term operations of $\mathbb{A}$.

Algebras allow for a more general definition of CSPs that is used here. Let $\operatorname{CSP}(\mathbb{A})$ denote the class of nonuniform $\operatorname{CSPs}\{\operatorname{CSP}(\Gamma) \mid \Gamma \subseteq \operatorname{lnv}(F)\}$, where $\operatorname{lnv}(F)$ denotes the set of all relations invariant with respect to all operations from $F$. Note that the tractability of $\operatorname{CSP}(\mathbb{A})$ can be understood in two ways: as the existence of a polynomial-time algorithm for every $\operatorname{CSP}(\Gamma)$ from this class, or as the existence of a uniform polynomial-time algorithm for all such problems. One of the implications of our results is that these two types of tractability are equivalent. From the formal standpoint we will use the stronger one.

The main structural elements are subalgebras, congruences, and quotient algebras. For $B \subseteq A$ and an operation $f$ on $A$ by $\left.f\right|_{B}$ we denote the restriction of $f$ on $B$. Algebra $\mathbb{B}=\left(B,\left\{\left.f\right|_{B} \mid f \in F\right\}\right)$ is called a subalgebra of $\mathbb{A}$ if $f\left(b_{1}, \ldots, b_{k}\right) \in B$ for any $b_{1}, \ldots, b_{k} \in B$ and any $f \in F$.

Congruences play a very significant role in our algorithm, and we discuss them in more details. A congruence is an equivalence relation $\theta \in \operatorname{lnv}(F)$. This means that for any operation $f \in F$ and any $\left(a_{1}, b_{1}\right), \ldots,\left(a_{k}, b_{k}\right) \in \theta$ it holds $\left(f\left(a_{1}, \ldots, a_{k}\right), f\left(b_{1}, \ldots, b_{k}\right)\right) \in \theta$. Therefore it is possible to define an algebra on $A /{ }_{\theta}$, the set of $\theta$-blocks, by setting $f /{ }_{\theta}\left(a_{1}^{\theta}, \ldots, a_{k}^{\theta}\right)=\left(f\left(a_{1}, \ldots, a_{k}\right)\right) /{ }_{\theta}$ for $a_{1}, \ldots, a_{k} \in A$, where $a^{\theta}$ denotes the $\theta$-block containing $a$. The resulting algebra $\mathbb{A} / \theta$ is called the quotient algebra modulo $\theta$.

The following are examples of congruences and quotient algebras.

- Let $\mathbb{A}$ be any algebra. Then the equality relation $\underline{0}_{\mathbb{A}}$ and the full binary relation $\underline{1}_{\mathbb{A}}$ on $\mathbb{A}$ are congruences of $\mathbb{A}$. The quotient algebra $\mathbb{A} / \underline{\underline{0}}_{\mathbb{A}}$ is $\mathbb{A}$ itself, while $\mathbb{A} / \underline{1}_{\mathbb{A}}$ is a 1-element algebra.

- Let $\mathbb{S}_{n}$ be the permutation group on an $n$-element set and binary relation $\theta$ is given by: $(a, b) \in \theta$ for $a, b \in \mathbb{S}_{n}$ if and only if $a$ and $b$ have the same parity as permutations. Then $\theta$ is a congruence of $\mathbb{S}_{n}$ and $\mathbb{S}_{n} /{ }_{\theta}$ is the 2-element group.

- Let $\mathbb{L}_{n}$ be an $n$-dimensional vector space and $\mathbb{L}^{\prime}$ its $k$-dimensional subspace. The binary relation $\pi$ given by: $(\bar{a}, \bar{b}) \in \pi$ if and only if $\bar{a}, \bar{b}$ have the same orthogonal projection on $\mathbb{L}^{\prime}$, is a congruence of $\mathbb{L}_{n}$ and $\mathbb{L}_{n} /{ }_{\pi}$ is $\mathbb{L}^{\prime}$.

The (ordered) set of all congruences of $\mathbb{A}$ is denoted by $\operatorname{Con}(\mathbb{A})$. This set is actually a lattice. By $\mathrm{HS}(\mathbb{A})$ we denote the set of all quotient algebras of all subalgebras of $\mathbb{A}$.

The results of [26] reduce the dichotomy conjecture to idempotent algebras. An algebra $\mathbb{A}=(A, F)$ is said to be idempotent if every operation $f \in F$ satisfies the equation $f(x, \ldots, x)=x$. If $\mathbb{A}$ is idempotent, then all the constant relations 
$\{(a)\}$ are invariant under $F$. Therefore studying CSPs over idempotent algebras is the same as studying the CSPs that allow all constant relations. Another useful property of idempotent algebras is that every block of every its congruence is a subalgebra. We now can state the algebraic version of the dichotomy theorem.

Theorem 4 For a finite idempotent algebra $\mathbb{A}$ the following are equivalent:

(1) $\operatorname{CSP}(\mathbb{A})$ is solvable in polynomial time;

(2) $\mathbb{A}$ has a weak near-unanimity term operation;

(3) every algebra from $\mathrm{HS}(\mathbb{A})$ has a nontrivial term operation (that is not a projection, an operation of the form $\left.f\left(x_{1}, \ldots, x_{k}\right)=x_{i}\right)$;

Otherwise $\operatorname{CSP}(\mathbb{A})$ is NP-complete.

The hardness part of this theorem is proved in [26]; the equivalence of (2) and (3) was proved in [25] and [54]. The equivalence of (1) to (2) and (3) is the main result of this paper. In the rest of the paper we assume all algebras to satisfy conditions (2),(3).

\subsection{Bounded width and the few subpowers algorithm}

Leaving aside occasional combinations thereof, there are only two standard types of algorithms solving the CSP. In this section we give a brief introduction into them.

CSPs of bounded width. Algorithms of the first kind are based on the idea of local propagation, that is formally described below. By $[n]$ we denote the set $\{1, \ldots, n\}$. For sets $A_{1}, \ldots, A_{n}$ tuples from $A_{1} \times \cdots \times A_{n}$ are denoted in boldface, say, $\mathbf{a}$; the $i$ th component of $\mathbf{a}$ is referred to as $\mathbf{a}[i]$. An $n$-ary relation $R$ over sets $A_{1}, \ldots, A_{n}$ is any subset of $A_{1} \times \cdots \times A_{n}$. For $I=\left\{i_{1}, \ldots, i_{k}\right\} \subseteq[n]$ by $\operatorname{pr}_{I} \mathbf{a}, \operatorname{pr}_{I} R$ we denote the projections $\operatorname{pr}_{I} \mathbf{a}=\left(\mathbf{a}\left[i_{1}\right], \ldots, \mathbf{a}\left[i_{k}\right]\right), \operatorname{pr}_{I} R=\left\{\operatorname{pr}_{I} \mathbf{a} \mid\right.$ $\mathbf{a} \in R\}$ of tuple a and relation $R$. If $\operatorname{pr}_{i} R=A_{i}$ for each $i \in[n]$, relation $R$ is said to be a subdirect product of $A_{1} \times \cdots \times A_{n}$.

Let $\mathcal{P}=(V, \mathcal{C})$ be a CSP instance. For $W \subseteq V$ by $\mathcal{P}_{W}$ we denote the restriction of $\mathcal{P}$ onto $W$, that is, the instance $\left(W, \mathcal{C}_{W}\right)$, where for each $C=\langle\mathbf{s}, R\rangle \in \mathcal{C}$, the set $\mathcal{C}_{W}$ includes the constraint $C_{W}=\left\langle\mathbf{s} \cap W, \operatorname{pr}_{\mathbf{s} \cap W} R\right\rangle$. The set of solutions of $\mathcal{P}_{W}$ will be denoted by $\mathcal{S}_{W}$.

Unary solutions, that is, when $|W|=1$ play a special role. As is easily seen, for $v \in V$ the set $\mathcal{S}_{v}$ is just the intersections of unary projections $\operatorname{pr}_{v} R$ of constraints whose scope contains $v$. One may always assume that allowed values for a variable $v \in V$ is the set $\mathcal{S}_{v}$. We call this set the domain of $v$ and assume that CSP instances 
may have different domains, which nevertheless are always subalgebras or quotient algebras of the original algebra $\mathbb{A}$. It will be convenient to denote the domain of $v$ by $\mathbb{A}_{v}$. The domain $\mathbb{A}_{v}$ may change as a result of transformations of the instance. Instance $\mathcal{P}$ is said to be 1 -minimal if for every $v \in V$ and every constraint $C=\langle\mathbf{s}, R\rangle \in \mathcal{C}$ such that $v \in \mathbf{s}$, it holds $\operatorname{pr}_{v} R=\mathbb{A}_{v}$.

Instance $\mathcal{P}$ is said to be (2,3)-consistent if it has a (2,3)-strategy, that is, a collection of relations $R^{X}, X \subseteq V,|X|=2$ satisfying the following conditions:

- for every $X \subseteq V$ with $|X| \leq 2$ and every $C=\langle\mathbf{s}, R\rangle, \operatorname{pr}_{\mathbf{s} \cap X} R^{X} \subseteq \operatorname{pr}_{\mathbf{s} \cap X} R$;

- for every $X=\{u, v\} \subseteq V$, any $w \in V-X$, and any $(a, b) \in R^{X}$, there is $c \in \mathbb{A}_{w}$ such that $(a, c) \in R^{\{u, w\}}$ and $(b, c) \in R^{\{v, w\}}$.

We will always assume that a (2,3)-consistent instance has a constraint $C^{X}=$ $\left\langle X, \mathcal{S}_{X}\right\rangle$ for every $X \subseteq V,|X|=2$. Then clearly $R^{X} \subseteq \mathcal{S}_{X}$. Let the collection of relations $R^{X}$ be denoted by $\mathcal{R}$. A tuple a whose entries are indexed with elements of $W \subseteq V$ and such that $\operatorname{pr}_{X} \mathbf{a} \in R^{X}$ for any $X \subseteq W,|X|=2$, will be called $\mathcal{R}$-compatible. If a (2,3)-consistent instance $\mathcal{P}$ with a (2,3)-strategy $\mathcal{R}$ satisfies the additional condition

- for every constraint $C=\langle\mathbf{s}, R\rangle$ of $\mathcal{P}$ every tuple $\mathbf{a} \in R$ is $\mathcal{R}$-compatible,

it is called (2,3)-minimal. For $k \in \mathbb{N},(k, k+1)$-strategies, $(k, k+1)$-consistency, and $(k, k+1)$-minimality are defined in a similar way replacing 2,3 with $k, k+1$.

Instance $\mathcal{P}$ is said to be minimal (or globally minimal) if for every $C=\langle\mathbf{s}, R\rangle \in$ $\mathcal{C}$ and every a $\in R$ there is a solution $\varphi \in \mathcal{S}$ such that $\varphi(\mathbf{s})=\mathbf{a}$.

Any instance can be transformed to a 1-minimal, $(2,3)$-consistent, or $(2,3)$ minimal instance in polynomial time using the standard constraint propagation algorithms (see, e.g. [32]). These algorithms work by changing the constraint relations and the domains of the variables eliminating some tuples and elements from them. We call such a process tightening the instance. It is important to notice that if the original instance belongs to $\operatorname{CSP}(\mathbb{A})$ for some algebra $\mathbb{A}$, that is, all its constraint relations are invariant under the term operations of $\mathbb{A}$, the constraint relations obtained by propagation algorithms are also invariant under term operations of $\mathbb{A}$, and so the resulting instance also belongs to $\operatorname{CSP}(\mathbb{A})$. Establishing minimality amounts to solving the problem and therefore not always can be easily done.

If a constraint propagation algorithm solves a CSP, the problem is said to be of bounded width. More precisely, $\operatorname{CSP}(\Gamma)$ (or $\operatorname{CSP}(\mathbb{A}))$ is said to have bounded width if for some $k$ every $(k, k+1)$-minimal instance from $\operatorname{CSP}(\Gamma)($ or $\operatorname{CSP}(\mathbb{A}))$ has a solution. Problems of bounded width are very well studied, see the older survey [27] and a more recent paper [4].

Theorem $5([4,21,47])$ For an idempotent algebra $\mathbb{A}$ the following are equivalent: 
(1) $\operatorname{CSP}(\mathbb{A})$ has bounded width;

(2) every (2,3)-minimal instance from $\operatorname{CSP}(\mathbb{A})$ has a solution;

(3) $\mathbb{A}$ has a weak near-unanimity term of arity $k$ for every $k \geq 3$;

(4) every algebra $\mathrm{HS}(\mathbb{A})$ has a nontrivial operation, and none of them is equivalent to a module.

Omitting semilattice edges and the few subpowers property. The second type of CSP algorithms can be viewed as a generalization of Gaussian elimination, although, it utilizes just one property also used by Gaussian elimination: the set of solutions of a system of linear equations or a CSP has a set of generators of size linear in the number of variables. The property that for every instance $\mathcal{P}$ of $\operatorname{CSP}(\mathbb{A})$ its solution space $\mathcal{S}_{\mathcal{P}}$ has a set of generators of polynomial size is nontrivial, because there are only exponentially many such sets, while, as is easily seen CSPs with $n$ variables may have up to double exponentially many different sets of solutions. Formally, an algebra $\mathbb{A}=(A, F)$ has few subpowers if for every $n$ there are only exponentially many $n$-ary relations in $\operatorname{lnv}(F)$.

Algebras with few subpowers are well studied, completely characterized, and the CSP over such an algebra has a polynomial-time solution algorithm, see, [11, 40]. In particular, such algebras admit a characterization in terms of the existence of a term operation with special properties, an edge term. We however need only a subclass of algebras with few subpowers that appeared in [21] and is defined as follows.

A pair of elements $a, b \in \mathbb{A}$ is said to be a semilattice edge if there is a binary term operation $f$ of $\mathbb{A}$ such that $f(a, a)=a$ and $f(a, b)=f(b, a)=f(b, b)=b$, that is, $f$ is a semilattice operation on $\{a, b\}$.

Proposition $6([21])$ If an idempotent algebra $\mathbb{A}$ has no semilattice edges, it has few subpowers, and therefore $\operatorname{CSP}(\mathbb{A})$ is solvable in polynomial time.

Semilattice edges have other useful properties including the following one that we use for reducing a CSP to smaller problems.

Lemma 7 ([20]) For any idempotent algebra $\mathbb{A}$ there is a binary term operation $x y$ of $\mathbb{A}$ (think multiplication) such that $x y$ is a semilattice operation on any semilattice edge and for any $a, b \in \mathbb{A}$ either $a b=a$ or $\{a, a b\}$ is a semilattice edge.

\section{Solving CSPs}

\subsection{Congruence separation and centralizers}

In this section we introduce two of the key ingredients of the algorithm. 
Separating congruences. Unlike the vast majority of the literature on the algebraic approach to the CSP we use not only term operations, but also polynomial operations of an algebra. It should be noted however that the first to use polynomials for CSP algorithms was Maroti in [53]. We make use of some ideas from that paper in the next section.

Let $f\left(x_{1}, \ldots, x_{k}, y_{1}, \ldots, y_{\ell}\right)$ be a $k+\ell$-ary term operation of an algebra $\mathbb{A}$ and $b_{1}, \ldots, b_{\ell} \in \mathbb{A}$. The operation $g\left(x_{1}, \ldots, x_{k}\right)=f\left(x_{1}, \ldots, x_{k}, b_{1}, \ldots, b_{\ell}\right)$ is called a polynomial of $\mathbb{A}$. The name 'polynomial' refers to usual polynomials. Indeed, if $\mathbb{A}$ is a ring, its polynomials as just defined are the same as polynomials in the regular sense. A polynomial that depends on only one variable is said to be a unary polynomial.

While polynomials of $\mathbb{A}$ do not have to be polymorphisms of relations from $\operatorname{Inv}(F)$, congruences and unary polynomials are in a special relationship. More precisely, an equivalence relation over $\mathbb{A}$ is a congruence if and only if it is preserved by all the unary polynomials of $\mathbb{A}$.

Let $\mathbb{A}$ be an algebra. For $\alpha, \beta \in \operatorname{Con}(\mathbb{A})$ we write $\alpha \prec \beta$ if $\alpha<\beta$ and $\alpha \leq \gamma \leq \beta$ in $\operatorname{Con}(\mathbb{A})$ implies $\gamma=\alpha$ or $\gamma=\beta$; if this is the case we call $(\alpha, \beta)$ a prime interval in $\operatorname{Con}(\mathbb{A})$. Let $\alpha \prec \beta$ and $\gamma \prec \delta$ be prime intervals in $\operatorname{Con}(\mathbb{A})$. We say that $\alpha \prec \beta$ can be separated from $\gamma \prec \delta$ if there is a unary polynomial $f$ of $\mathbb{A}$ such that $f(\beta) \nsubseteq \alpha$, but $f(\delta) \subseteq \gamma$. The polynomial $f$ in this case is said to separate $\alpha \prec \beta$ from $\gamma \prec \delta$.

In a similar way separation can be defined for prime intervals in different coordinate positions of a relation. Let $R$ be a subdirect product of $\mathbb{A}_{1} \times \cdots \times \mathbb{A}_{n}$. Then $R$ is also an algebra and its polynomials can be defined in the same way. Let $i, j \in[n]$ and let $\alpha \prec \beta, \gamma \prec \delta$ be prime intervals in $\operatorname{Con}\left(\mathbb{A}_{i}\right)$ and $\operatorname{Con}\left(\mathbb{A}_{j}\right)$, respectively. Interval $\alpha \prec \beta$ can be separated from $\gamma \prec \delta$ if there is a unary polynomial $f$ of $R$ such that $f(\beta) \nsubseteq \alpha$ but $f(\delta) \subseteq \gamma$ (note that the actions of $f$ on $\mathbb{A}_{i}, \mathbb{A}_{j}$ are polynomials of those algebras).

The binary relation 'cannot be separated' on the set of prime intervals of an algebra or factors of a relation is easily seen to be reflexive and transitive. Under certain mild conditions it can also be shown to be symmetric in a certain sense (Lemma 46), and so for the purpose of our algorithm it can be treated as an equivalence relation.

Quasi-Centralizers. The second ingredient introduced here is the notion of quasicentralizer of a prime interval of congruences. It is similar to the centralizer as it is defined in commutator theory, albeit the exact relationship between the two concepts is not quite clear, and so we name differently for safety.

For an algebra $\mathbb{A}$, a term operation $f\left(x, y_{1}, \ldots, y_{k}\right)$, and $\mathbf{a} \in \mathbb{A}^{k}$, let $f^{\mathbf{a}}(x)=$ 
$f(x, \mathbf{a})$. Let $\alpha, \beta \in \operatorname{Con}(\mathbb{A}), \alpha \leq \beta$, and let $\zeta(\alpha, \beta) \subseteq \mathbb{A}^{2}$ denote the following binary relation: $(a, b) \in \zeta(\alpha, \beta)$ if an only if, for any term operation $f\left(x, y_{1}, \ldots, y_{k}\right)$, any $i \in[k]$, and any $\mathbf{a}, \mathbf{b} \in \mathbb{A}^{k}$ such that $\mathbf{a}[i]=a, \mathbf{b}[i]=b$, and $\mathbf{a}[j]=\mathbf{b}[j]$ for $j \neq i$, it holds $f^{\mathbf{a}}(\beta) \subseteq \alpha$ if and only if $f^{\mathbf{b}}(\beta) \subseteq \alpha$. The relation $\zeta(\alpha, \beta)$ is always a congruence of $\mathbb{A}$ (Lemma 51) and its effect on the structure of algebra $\mathbb{A}$ is illustrated by the following statement.

Lemma 8 Let $\zeta(\alpha, \beta)=\underline{1}_{\mathbb{A}}, a, b, c \in \mathbb{A}$ and $(b, c) \in \beta$. Then $(a b, a c) \in \alpha$.

Fig. 1(a),(b) shows the effect of large quasi-centralizers on the structure of algebra $\mathbb{A}$. Dots there represent $\alpha$-blocks (assume $\alpha$ is the equality relation), ovals represent $\beta$-blocks, let they be $B$ and $C$, and such that there is at least one semilattice edge between $B$ and $C$. If $\zeta(\alpha, \beta)$ is the full relation, Lemmas 7 and 8 imply that for any $a \in B$ and any $b, c \in C$ we have $a b=a c$, and so $a b$ is the only element of $C$ such that $\{a, a b\}$ is a semilattice edge (represented by arrows). In other words, we have a mapping that can also be shown injective from $B$ to $C$. We will use this mapping to lift any solution with a value from $B$ to a solution with a value from $C$.

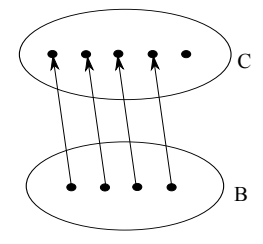

(a) $\zeta(\alpha, \beta)$ is the full relation

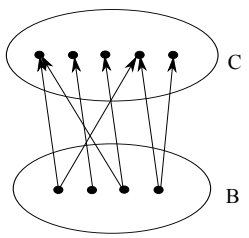

(b) $\zeta(\alpha, \beta)$ is not the full relation

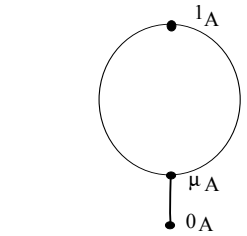

(c) Congruence lattice of a subdirectly irreducible algebra

Figure 1:

\subsection{The algorithm}

We have seen in the previous section that big centralizers impose strong restrictions on the structure of an algebra. We start this section showing that small centralizers restrict the structure of CSPs.

Decomposition of CSPs. Let $R$ be a binary relation, a subdirect product of $\mathbb{A} \times$ $\mathbb{B}$, and $\alpha \in \operatorname{Con}(\mathbb{A}), \gamma \in \operatorname{Con}(\mathbb{B})$. Relation $R$ is said to be $\alpha \gamma$-aligned if, for any $(a, c),(b, d) \in R,(a, b) \in \alpha$ if and only if $(c, d) \in \gamma$. This means that if $A_{1}, \ldots, A_{k}$ are the $\alpha$-blocks of $\mathbb{A}$, then there are also $k \gamma$-blocks of $\mathbb{B}$ and they can be labeled $B_{1}, \ldots, B_{k}$ in such a way that

$$
R=\left(R \cap\left(A_{1} \times B_{1}\right)\right) \cup \cdots \cup\left(R \cap\left(A_{k} \times B_{k}\right)\right) .
$$


Lemma 9 Let $R, \mathbb{A}, \mathbb{B}$ be as above and $\alpha, \beta \in \operatorname{Con}(\mathbb{A}), \gamma, \delta \in \operatorname{Con}(\mathbb{B})$, with $\alpha \prec \beta, \gamma \prec \delta$. If $(\alpha, \beta)$ and $(\gamma, \delta)$ cannot be separated, then $R$ is $\zeta(\alpha, \beta) \zeta(\gamma, \delta)$ aligned.

Lemma 9 provides a way to decompose CSP instances. Let $\mathcal{P}=(V, \mathcal{C})$ be a (2,3)-minimal instance from $\operatorname{CSP}(\mathbb{A})$, in particular, $\mathcal{C}$ contains a constraint $C^{\{v, w\}}=\left\langle(v, w), R^{\{v, w\}}\right\rangle$ for every $v, w \in V$, and these relations form a (2,3)strategy for $\mathcal{P}$. Due to $(2,3)$-minimality the domain of variables from $V$ do not have to be $\mathbb{A}$ itself, but can be subalgebras of $\mathbb{A}$. Recall that $\mathbb{A}_{v}$ denotes the domain of $v \in V$. Also, let $W \subseteq V$ and congruences $\alpha_{v}, \beta_{v} \in \operatorname{Con}\left(\mathbb{A}_{v}\right)$ for $v \in W$ be such that $\alpha_{v} \prec \beta_{v}$, and for any $v, w \in W$ the intervals $\left(\alpha_{v}, \beta_{v}\right)$ and $\left(\alpha_{w}, \beta_{w}\right)$ cannot be separated in $R^{\{v, w\}}$.

Denoting $\zeta_{v}=\zeta\left(\alpha_{v}, \beta_{v}\right)$ we see that there is a one-to-one correspondence between $\zeta_{v}$ and $\zeta_{w}$ blocks of $\mathbb{A}_{v}$ and $\mathbb{A}_{w}$. Moreover, by (2,3)-minimality these correspondences are consistent, that is, if $u, v, w \in W$ and $B_{u}, B_{v}, B_{w}$ are $\zeta_{u^{-}}$, $\zeta_{v^{-}}$and $\zeta_{w}$-blocks, respectively, such that $R^{\{u, v\}} \cap\left(B_{u} \times B_{v}\right) \neq \varnothing$ and $R^{\{v, w\}} \cap$ $\left(B_{v} \times B_{w}\right) \neq \varnothing$, then $R^{\{u, w\}} \cap\left(B_{u} \times B_{w}\right) \neq \varnothing$. This means that $\mathcal{P}_{W}$ can be split into several instances, whose domains are $\zeta_{v}$-blocks.

Lemma 10 Let $\mathcal{P}, W, \alpha_{v}, \beta_{v}$ be as above. Then $\mathcal{P}_{W}$ can be decomposed into a collection of instances $\mathcal{P}_{1}, \ldots, \mathcal{P}_{k}, \mathcal{P}_{i}=\left(W, \mathcal{C}_{i}\right)$ such that every solution of $\mathcal{P}_{W}$ is a solution of one of the $\mathcal{P}_{i}$ and for every $v \in V$ its domain in $\mathcal{P}_{i}$ is a $\zeta_{v}$-block.

Irreducibility. In order to formulate the algorithm properly we need one more transformation of algebras. An algebra $\mathbb{A}$ is said to be subdirectly irreducible if the intersection of all its nontrivial (different from the equality relation) congruences is nontrivial. This smallest nontrivial congruence $\mu_{\mathbb{A}}$ is called the monolith of $\mathbb{A}$, see Fig. 1(c). It is a folklore observation that any CSP instance can be transformed in polynomial time to an instance, in which the domain of every variable is a subdirectly irreducible algebra. We will assume this property of all the instances we consider.

Block-minimality. Lemma 10 allows one to establish a much stronger version of local consistency, block-minimality; in fact, it is not local anymore. The definitions below are designed in such a way that to allow for an efficient procedure to establish block-minimality. This is achieved either by allowing for decomposing a subinstance into instances over smaller domains as in Lemma 10, or by replacing large domains with their quotient algebras.

Let $\alpha_{v}$ be a congruence of $\mathbb{A}_{v}$ for each $v \in V$. By $\mathcal{P} / \bar{\alpha}$ we denote the instance $\left(V, \mathcal{C}^{\bar{\alpha}}\right)$ constructed as follows: the domain of $v \in V$ is $\mathbb{A}_{v} / \alpha_{v}$; for every 
constraint $C=\langle\mathbf{s}, R\rangle \in \mathcal{C}$, the set $\mathcal{C}^{\bar{\alpha}}$ includes the constraint $\left\langle\mathbf{s}, R / \bar{\alpha}_{\mathbf{s}}\right\rangle$, where $\mathbf{s}=\left(v_{1}, \ldots, v_{k}\right)$ and $R / \bar{\alpha}=\left\{\left(\mathbf{a}\left[v_{1}\right]^{\alpha_{v_{1}}}, \ldots, \mathbf{a}\left[v_{k}\right]^{\alpha_{v_{k}}}\right) \mid \mathbf{a} \in R\right\}$.

Let $\mathcal{P}=(V, \mathcal{C})$ be a $(2,3)$-minimal instance and $\left\{R^{X}|X \subseteq V| X \mid,=2\right\}$ is its (2,3)-strategy. Let $\bar{\beta}=\left(\beta_{v}\right)_{v \in V}, \beta_{v} \in \operatorname{Con}\left(\mathbb{A}_{v}\right), v \in V$, be a collection of congruences. Let $\mathcal{W}^{\mathcal{P}}(\bar{\beta})$ denote the set of triples $(v, \alpha, \beta)$ such that $v \in V$, $\alpha, \beta \in \operatorname{Con}\left(\mathbb{A}_{v}\right)$, and $\alpha \prec \beta \leq \beta_{v}$. Also, $\mathcal{W}^{\mathcal{P}}$ denotes $\mathcal{W}^{\mathcal{P}}(\bar{\beta})$ when $\beta_{v}$ is the full relation for all $v \in V$. We will omit the superscript $\mathcal{P}$ whenever it is clear from the context.

For every $(v, \alpha, \beta) \in \mathcal{W}(\bar{\beta})$, let $W_{v, \alpha \beta, \bar{\beta}}$ denote the set of variables $w \in V$ such that $(\alpha, \beta)$ and $(\gamma, \delta)$ cannot be separated in $R^{v w}$ for some $\gamma, \delta \in \operatorname{Con}\left(\mathbb{A}_{w}\right)$ with $(w, \gamma, \delta) \in \mathcal{W}^{\mathcal{P}}(\bar{\beta})$. Let $\mathcal{W}^{\prime}(\bar{\beta})$ (and respectively $\mathcal{W}^{\prime}$ ) denote the set of triples $(v, \alpha, \beta) \in \mathcal{W}(\bar{\beta})$ (respectively, from $\mathcal{W}$ ), for which $\zeta(\alpha, \beta)$ is the full relation.

We say that algebra $\mathbb{A}_{v}$ is semilattice free if it does not contain semilattice edges. Let $\operatorname{size}(\mathcal{P})$ denote the maximal size of domains of $\mathcal{P}$ that are not semilattice free and $\operatorname{MAX}(\mathcal{P})$ be the set of variables $v \in V$ such that $\left|\mathbb{A}_{v}\right|=\operatorname{size}(\mathcal{P})$ and $\mathbb{A}_{v}$ is not semilattice free. For an instance $\mathcal{P}$ we say that an instance $\mathcal{P}^{\prime}$ is strictly smaller than instance $\mathcal{P}$ if $\operatorname{size}\left(\mathcal{P}^{\prime}\right)<\operatorname{size}(\mathcal{P})$. For $Y \subseteq V$ let $\mu_{v}^{Y}=\mu_{v}$ if $v \in Y$ and $\mu_{v}^{Y}=\underline{0}_{v}$ otherwise.

Instance $\mathcal{P}$ is said to be block-minimal if for every $(v, \alpha, \beta) \in \mathcal{W}$ (here $\beta_{v}=$ $\left.\underline{1}_{v}, v \in V\right)$ the following conditions hold:

(BM1) for every $C=\langle\mathbf{s}, R\rangle \in \mathcal{C}$ the problem $\mathcal{P}_{W_{v, \alpha \beta}, \bar{\beta}}$ if $(v, \alpha, \beta) \notin \mathcal{W}^{\prime}$, and the problem $\mathcal{P}_{W_{v, \alpha \beta}, \bar{\beta}} / \bar{\mu}^{Y}$ otherwise, where $Y=\operatorname{MAX}(\mathcal{P})-\mathbf{s}$, is minimal;

(BM2) if $(v, \alpha, \beta) \in \mathcal{W}^{\prime}$, then for every $(w, \gamma, \delta) \in \mathcal{W}-\mathcal{W}^{\prime}$ the problem $\mathcal{P}_{W_{v, \alpha \beta, \bar{\beta}}} / \bar{\mu}^{Y}$, where $Y=\operatorname{MAX}(\mathcal{P})-\left(W_{v, \alpha \beta, \bar{\beta}} \cap W_{w, \gamma \delta, \bar{\beta}}\right)$ is minimal.

Observe that $W_{v, \alpha \beta, \bar{\beta}}$ can be large, even equal to $V$. However if $(v, \alpha, \beta) \notin \mathcal{W}^{\prime}$ by Lemma 10 the problem $\mathcal{P}_{W_{v, \alpha \beta, \bar{\beta}}}$ splits into a union of disjoint problems over smaller domains, and so its minimality can be established by recursing to strictly smaller problems. On the other hand, if $(v, \alpha, \beta) \in \mathcal{W}^{\prime}$ then $\mathcal{P}_{W_{v, \alpha \beta} \bar{\beta}}$ may not be decomposable. Since we need an efficient procedure of establishing blockminimality, this explains the complications introduced in (BM1),(BM2). In the first case $\mathcal{P}_{W_{v, \alpha \beta}, \bar{\beta}} / \bar{\mu}^{Y}$ can be solved for each tuple $\mathbf{a} \in R$. Taking the quotient algebras of the domains guarantees that we recurse to strictly smaller instances. In the second case $\mathcal{P}_{W_{v, \alpha \beta}, \bar{\beta}} \cap W_{w, \gamma \delta, \bar{\beta}} / \bar{\mu}^{Y}$ is decomposable, and we branch on those strictly smaller subproblems.

Lemma 11 Let $\mathcal{P}=(V, \mathcal{C})$ be a (2,3)-minimal instance. Then $\mathcal{P}$ can be transformed to an equivalent block-minimal instance $\mathcal{P}^{\prime}$ by solving a quadratic number of strictly smaller CSPs. 
The algorithm. In the algorithm we distinguish three cases depending on semilattice edges and quasi-centralizers of the domains of variables. In each case we employ different methods of solving or reducing the instance to a strictly smaller one.

Let $\mathcal{P}=(V, \mathcal{C})$ be a subdirectly irreducible $(2,3)$-minimal instance. Let $\operatorname{Center}(\mathcal{P})$ denote the set of variables $v \in V$ such that $\zeta\left(\underline{0}_{v}, \mu_{v}\right)=\underline{1}_{v}$. Let $\mu_{v}^{*}=\mu_{v}$ if $v \in \operatorname{MAX}(\mathcal{P}) \cap \operatorname{Center}(\mathcal{P})$ and $\mu_{v}^{*}=\underline{0}_{v}$ otherwise.

Semilattice free domains. If no domain of $\mathcal{P}$ contains a semilattice edge then by Proposition $6 \mathcal{P}$ can be solved in polynomial time, using the few subpowers algorithm, as shown in [40, 21].

Trivial centralizers. If $\mu_{v}^{*}=\underline{0}_{v}$ for all $v \in V$, block-minimality guarantees the existence of a solution, and we can use Lemma 11 to solve the instance.

Theorem 12 If $\mathcal{P}$ is subdirectly irreducible, (2,3)-minimal, block-minimal, and $\operatorname{MAX}(\mathcal{P}) \cap \operatorname{Center}(\mathcal{P})=\varnothing$, then $\mathcal{P}$ has a solution.

Nontrivial centralizers. Suppose that $\operatorname{MAX}(\mathcal{P}) \cap \operatorname{Center}(\mathcal{P}) \neq \varnothing$. In this case we consider the problem $\mathcal{P} / \bar{\mu}^{*}$. For this problem either $\operatorname{size}\left(\mathcal{P} / \bar{\mu}^{*}\right)<\operatorname{size}(\mathcal{P})$, or $\operatorname{MAX}\left(\mathcal{P} / \bar{\mu}^{*}\right) \cap \operatorname{Center}\left(\mathcal{P} / \bar{\mu}^{*}\right)=\varnothing$; in either case it can be solved by the previous case or by recursion to a strictly smaller problem. We find a solution $\varphi$ of $\mathcal{P} / \bar{\mu}^{*}$ satisfying the following conditions. For every $v \in V$ such that $\mathbb{A}_{v}$ is not semilattice free there is $a \in \mathbb{A}_{v}$ such that $\{a, \varphi(v)\}$ is a semilattice edge if $\mu_{v}^{*}=\underline{0}_{v}$, or, if $\mu_{v}^{*}=\mu_{v}$, there is $b \in \varphi(v)$ such that $\{a, b\}$ is a semilattice edge. Then we apply the transformation of $\mathcal{P}$ suggested by Maroti in [53]. By $\mathcal{P} \cdot \varphi$ we denote the instance $\left(V, \mathcal{C}_{\varphi}\right)$ given by the rule: for every $\mathbf{C}=\langle\mathbf{s}, R\rangle \in \mathcal{C}$ the set $\mathcal{C}_{\varphi}$ contains a constraint $\langle\mathbf{s}, R \cdot \varphi\rangle$. To construct $R \cdot \varphi$ choose a tuple $\mathbf{b} \in R$ such that $\mathbf{b}[v]^{\mu_{v}^{*}}=\varphi(v)$ for all $v \in \mathbf{s}$; this is possible because $\varphi$ is a solution of $\mathcal{P} / \bar{\mu}^{*}$. Then set $R \cdot \varphi=\{\mathbf{a} \cdot \mathbf{b} \mid \mathbf{a} \in R\}$. By the results of [53] and Lemma 8 the instance $\mathcal{P} \cdot \varphi$ has a solution if and only if $\mathcal{P}$ does and $\operatorname{size}(\mathcal{P} \cdot \varphi)<\operatorname{size}(\mathcal{P})$.

Theorem 13 If $\mathcal{P} / \bar{\mu}^{*}$ is 1-minimal, then $\mathcal{P}$ can be reduced in polynomial time to a strictly smaller instance.

Comments on the algorithm. Using Lemma 11 and Theorems 12]13 it is not difficult to see that the algorithm runs in polynomial time. Indeed, every time it makes a recursive call it calls on a problem whose non-semilattice free domains have strictly smaller size, and therefore the depth of recursion is bounded by $|\mathbb{A}|$ if we are dealing with $\operatorname{CSP}(\mathbb{A})$. 


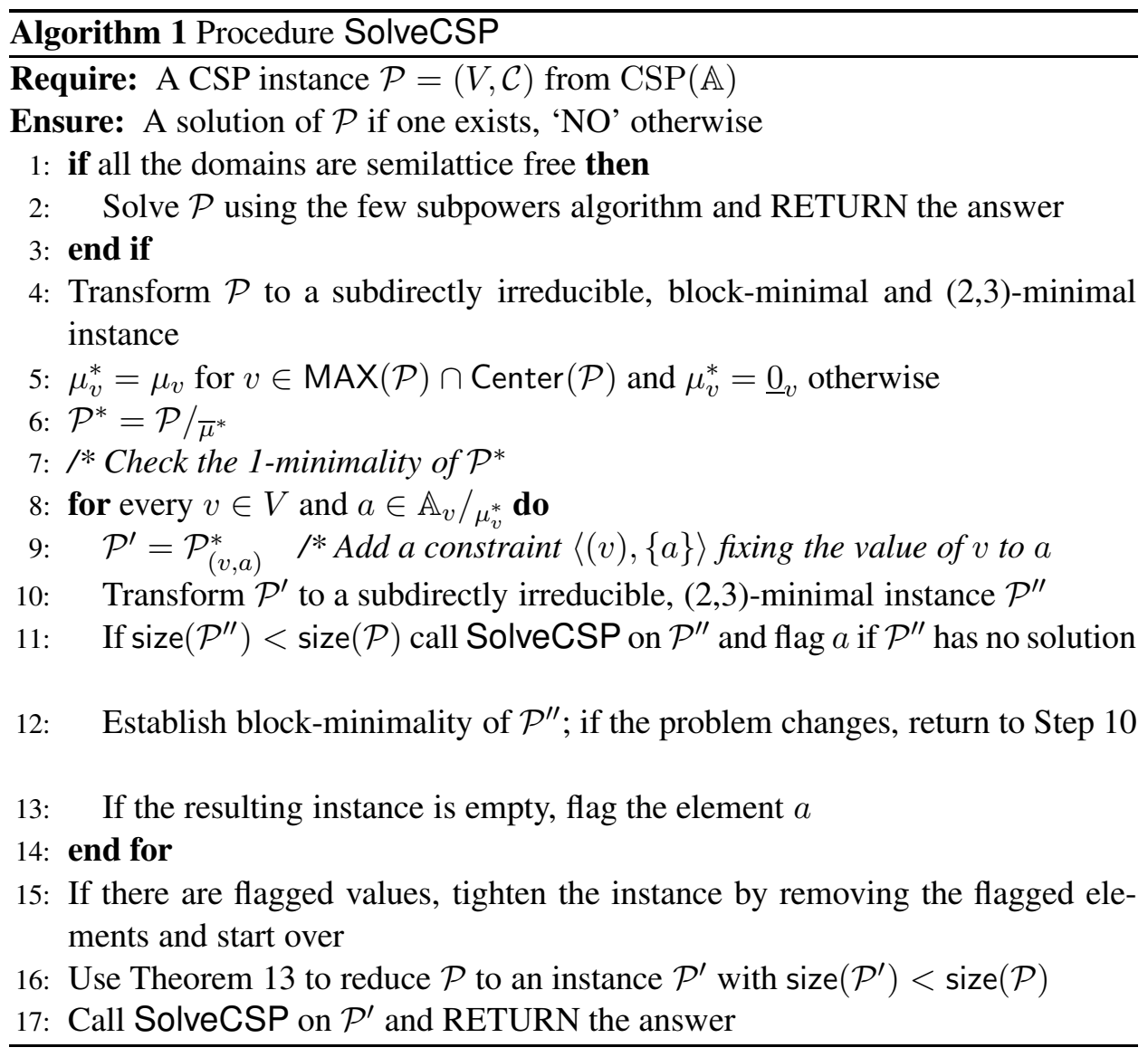


In order to prove Theorem 12 we introduce $\bar{\beta}$-strategies that are somewhat similar to $(2,3)$-strategies in the sense that they are also collections of relations defined through some sort of minimality condition and are consistent. We show how such constructions can be used to prove Theorem 12 .

Let $\mathcal{P}=(V, \mathcal{C})$ be a subdirectly irreducible, $(2,3)$-minimal and block-minimal instance. Let $\mathbb{A}_{v}$ denote the domain of $v \in V$. Also, let $\beta_{v} \in \operatorname{Con}\left(\mathbb{A}_{v}\right)$ and $B_{v}$ a $\beta_{v}$-block. Let $\mathcal{R}$ be a collection of relations $R_{C, v, \alpha \beta}$ for every $C=\langle\mathbf{s}, R\rangle \in \mathcal{C}$, $(v, \alpha, \beta) \in \mathcal{W}(\bar{\beta})$ and such that $S(C, v, \alpha \beta)=\mathbf{s} \cap W_{v, \alpha \beta, \bar{\beta}}$ is its set of coordinate positions. Similar to (2,3)-minimality a tuple a $\in \prod_{w \in X} \mathbb{A}_{x}$ for some $X \subseteq V$, is called $\mathcal{R}$-compatible if for any $C \in \mathcal{C}$ and $(v, \alpha, \beta) \in \mathcal{W}(\bar{\beta})$ it holds $\operatorname{pr}_{T} \mathbf{a} \in$ $\operatorname{pr}_{T} R_{C, v, \alpha \beta}$, where $T=X \cap S(C, v, \alpha \beta)$. Collection $\mathcal{R}$ is said to be a $\bar{\beta}$-strategy with respect to $\left(B_{v}\right)_{v \in V}$ if the following conditions hold for every $C=\langle\mathbf{s}, R\rangle \in \mathcal{C}$ and $(v, \alpha, \beta) \in \mathcal{W}(\bar{\beta})\left(\text { let } W=W_{v, \alpha \beta, \bar{\beta}}\right)^{2}$ :

(S1) the relations $R^{X, \mathcal{R}}$, where $R^{X, \mathcal{R}}$ consists of $\mathcal{R}$-compatible tuples from $R^{X}$ for $X \subseteq V,|X| \leq 2$, form a nonempty $(2,3)$-strategy for $\mathcal{P}$;

(S2) for every $(w, \gamma, \delta) \in \mathcal{W}(\bar{\beta})$ (let $\left.U=W_{w, \gamma \delta}\right)$ and every $\mathbf{a} \in \operatorname{pr}_{\mathbf{s} \cap W \cap U} R_{C, v, \alpha \beta}$ it holds: if $(w, \gamma, \delta) \notin \mathcal{W}^{\prime}$ then a extends to an $\mathcal{R}$-compatible solution of $\mathcal{P}_{U}$; otherwise if $(v, \alpha, \beta) \notin \mathcal{W}^{\prime}$ then a extends to an $\mathcal{R}$-compatible solution of $\mathcal{P}_{U} / \bar{\mu}^{Y_{1}}$ with $Y_{1}=\operatorname{MAX}(\mathcal{P})-(W \cap U)$; and if $(v, \alpha, \beta) \in \mathcal{W}^{\prime}$ then a extends to an $\mathcal{R}$-compatible solution of $\mathcal{P}_{U} / \bar{\mu}^{Y_{2}}$, where $Y_{2}=\operatorname{MAX}(\mathcal{P})-\mathbf{s}$;

(S3) $R \cap \prod_{w \in \mathbf{s}} B_{w} \neq \varnothing$ and for any $I \subseteq \mathbf{s}$ any $\mathcal{R}$-compatible tuple $\mathbf{a} \in \operatorname{pr}_{I} R$ extends to an $\mathcal{R}$-compatible tuple $\mathbf{b} \in R$.

Let $\mathcal{P}$ be a block-minimal instance, $\beta_{v}=\underline{1}_{v}$ and $B_{v}=\mathbb{A}_{v}$ for $v \in V$. Then as it is not hard to see the collection of relations $\mathcal{R}=\left\{R_{C, v, \alpha \beta} \mid(v, \alpha, \beta) \in\right.$ $\mathcal{W}(\bar{\beta}), C \in \mathcal{C}\}$ given by $R_{C, v, \alpha \beta}=\operatorname{pr}_{\mathbf{s} \cap W_{v, \alpha \beta, \bar{\beta}}} R$ for $C=\langle\mathbf{s}, R\rangle \in \mathcal{C}$ is a $\bar{\beta}$ strategy with respect to $\bar{B}$. Also, by (S3) a $\bar{\gamma}$-strategy with $\gamma_{v}=\underline{0}_{v}$ gives a solution of $\mathcal{P}$. Our goal is therefore to show that a $\bar{\beta}$-strategy for any $\bar{\beta}$ can be 'reduced', that is, transformed to a $\bar{\beta}^{\prime}$-strategy for some $\bar{\beta}^{\prime}<\bar{\beta}$. Note that this reduction of strategies is where the condition $\operatorname{MAX}(\mathcal{P}) \cap \operatorname{Center}(\mathcal{P})=\varnothing$ is used. Indeed, suppose that $\beta_{v}=\mu_{v}^{*}$. Then by conditions (S1)-(S3) we only have information about solutions to problems of the form $\mathcal{P}_{W} / \bar{\mu}^{*}$ or something very close to that. Therefore this barrier cannot be penetrated.

\footnotetext{
${ }^{2}$ These are the most important conditions that a $\bar{\beta}$-strategy has to satisfy. A complete and precise list of conditions can be found in Section 9.3 or [23].
} 


\section{Part II}

\section{Technicalities}

We start this part with preliminaries, where apart from additional definitions and notation, we remind some of the results of [16, 20, 21] related to colored graphs of algebras and relational structures and also some of their properties. In Sections 4.4 4.6 we advance these results a little further. Then in Section 5 we introduce in a more detailed way the method of separating factors in congruence lattices using polynomial operations of the algebra. This method constitutes the basis for our algorithm. Some preliminary versions of this approach can be found in [13, 22]. In Section 6 we introduce again and study the quasi-centralizer operator on congruence lattices that is similar to the well studied centralizer operator, although the precise relationship between the two is not quite clear. In particular, it allows to split certain CSPs into smaller ones. In Sections 7 and 8 we prove two important technical results

In Section 9 we give a description of the algorithm, and prove its running time and partially soundness. In very broad strokes the algorithm works as follows. If none of the domains of $\mathcal{P}$ contains a semilattice edge in the sense of colored graphs of algebras, then $\mathcal{P}$ can be solved by the few subpowers algorithm [11, 40], as shown in [21]. Otherwise in most cases the problem can be solved by establishing block-minimality similar to that in [22]. The problematic case when blockminimality does not provide a solution, or rather when it cannot be established is roughly speaking when the domains of the instance have nontrivial centers in the sense of the commutator theory. In this case we show in Section 9.2 that a solution of a problem $\mathcal{P}^{\prime}$ obtained from $\mathcal{P}$ by replacing some of its domains with quotient algebras modulo their centers allows one to reduce the number of semilattice edges in those domains, and we can recurse to an instance with smaller domains.

The key ingredient of our result is presented in Section 9.3. There for blockminimal instances we introduce strategies that are in certain aspects similar to strategies used to solve problems of bounded width, but allow us to approach general CSPs. Then in Section 10 we show, Theorem 68, that if for a CSP instance $\mathcal{P}$ satisfying the block-minimality conditions such a strategy exists, one can improve (tighten) the strategy to obtain a solution of the quotient problem $\mathcal{P}^{\prime}$ needed to reduce semilattice edges. This theorem is the most difficult and technically involved part of the proof. Tightening of a strategy works by (effectively) reducing domains of the CSP to a class of a maximal congruence, and then repeating the process as long as possible. The main cases of tightening considered are: when the interval formed by the maximal congruence used and the full congruence is Abelian, and when it is non-Abelian, Sections 10.1 and 10.2 , respectively. In the two cases we 
use quite different transformations of the strategy. In the Abelian case the argument is based on the rectangularity of relations understood in a general sense, while in the non-Abelian case the transformation is similar to that used for bounded width CSPs in [21].

\section{Preliminaries}

We expand upon many of the definitions and notation given in Part I. For the sake of convenience we also repeat some of the definitions given in Part $\llbracket$.

\subsection{Universal algebra and CSP: notation and agreements}

We assume familiarity with the basics of universal algebra and the algebraic approach to the CSP. For reference on universal algebra please use [29, 55]; for the algebraic approach see the recent survey [9] and earlier papers [5, 3, 26, 28, 27, 20].

By $[n]$ we denote the set $\{1, \ldots, n\}$. For sets $A_{1}, \ldots, A_{n}$ tuples from $A_{1} \times$ $\cdots \times A_{n}$ are denoted in boldface, say, $\mathbf{a}$; the $i$ th component of $\mathbf{a}$ is referred to as a $i$ ] . An $n$-ary relation $R$ over sets $A_{1}, \ldots, A_{n}$ is any subset of $A_{1} \times \cdots \times A_{n}$. For $I=\left\{i_{1}, \ldots, i_{k}\right\} \subseteq[n]$ by $\operatorname{pr}_{I} \mathbf{a}, \operatorname{pr}_{I} R$ we denote the projections $\operatorname{pr}_{I} \mathbf{a}=$ $\left(\mathbf{a}\left[i_{1}\right], \ldots, \mathbf{a}\left[i_{k}\right]\right), \operatorname{pr}_{I} R=\left\{\operatorname{pr}_{I} \mathbf{a} \mid \mathbf{a} \in R\right\}$ of tuple a and relation $R$. If $\operatorname{pr}_{i} R=A_{i}$ for each $i \in[n]$, relation $R$ is said to be a subdirect product of $A_{1} \times \cdots \times A_{n}$. It will be convenient to use $\bar{A}$ for $A_{1} \times \cdots \times A_{n}$, or for $\prod_{v \in V} A_{v}$ if the sets $V$ and $A_{v}$ are clear from the context. For $I \subseteq[n]$ or $I \subseteq V$ we will use $\bar{A}_{I}$, for $\prod_{i \in I} A_{i}$, or if $I$ is clear from the context just $\bar{A}$.

Algebras will be denoted by $\mathbb{A}, \mathbb{B}$ etc.; we often do not distinguish between subuniverses and subalgebras. For $B \subseteq \mathbb{A}$ the subalgebra generated by $B$ is denoted $\operatorname{Sg}(B)$. For $C \subseteq \mathbb{A}^{2}$ the congruence generated by $C$ is denoted $\operatorname{Cg}(C)$. The equality relation and the full congruence of algebra $\mathbb{A}$ are denoted $\underline{0}_{\mathbb{A}}$ and $\underline{1}_{\mathbb{A}}$, respectively. Often when we need to use one of these trivial congruences of an algebra indexed in some way, say, $\mathbb{A} i$, we write $\underline{0}_{i}, \underline{1}_{i}$ for $\underline{0}_{\mathbb{A}_{i}}, \underline{1}_{\mathbb{A}_{i}}$. The set of all polynomials (unary polynomials) of $\mathbb{A}$ is denoted by $\operatorname{Pol}(\mathbb{A})$ and $\operatorname{Pol}_{1}(\mathbb{A})$, respectively. We frequently use operations on subalgebras of direct products of algebras, say, $R \subseteq \mathbb{A}_{1} \times \cdots \times \mathbb{A}_{n}$. If $f$ is such an operation (say, $k$-ary) then we denote its component-wise action also by $f$, e.g. $f\left(a_{1}, \ldots, a_{k}\right)$ for $a_{1}, \ldots, a_{k} \in \mathbb{A}_{i}$. In the same way we denote the action of $f$ on projections of $R$, e.g. $f\left(\mathbf{a}_{1}, \ldots, \mathbf{a}_{k}\right)$ for $I \subseteq[n]$ and $\mathbf{a}_{1}, \ldots, \mathbf{a}_{k} \in \operatorname{pr}_{I} R$. What we mean will always be clear from the context. We use similar agreements for collections of congruences. If $\alpha_{i} \in \operatorname{Con}\left(\mathbb{A}_{i}\right)$ then $\bar{\alpha}$ denotes the congruence $\alpha_{1} \times \cdots \times \alpha_{n}$ of $R$. If $I \subseteq[n]$ we use $\bar{\alpha}_{I}$ to denote $\prod_{i \in I} \alpha_{i}$. If it does not lead to a confusion we write $\bar{\alpha}$ for $\bar{\alpha}_{I}$. Sometimes $\alpha_{i}$ 
are specified for $i$ from a certain set $I \subseteq[n]$, then by $\bar{\alpha}$ we mean the congruence $\prod_{i \in[n]} \alpha_{i}^{\prime}$ where $\alpha_{i}^{\prime}=\alpha_{i}$ if $i \in I$ and $\alpha_{i}^{\prime}$ is the equality relation otherwise. For example, if $\alpha \in \operatorname{Con}\left(\mathbb{A}_{1}\right)$ then $R / \alpha$ means the factor of $R$ modulo $\alpha \times \underline{0}_{2} \times \cdots \times \underline{0}_{n}$. For $\alpha, \beta \in \operatorname{Con}(\mathbb{A})$ we write $\alpha \prec \beta$ if $\alpha<\beta$ and $\alpha \leq \gamma \leq \beta$ in $\operatorname{Con}(\mathbb{A})$ implies $\gamma=\alpha$ or $\gamma=\beta$. In this paper all algebras are finite, idempotent and omit type $\mathbf{1}$.

The (nonuniform) Constraint Satisfaction Problem (CSP) associated with a relational structure $\mathbf{B}$ is the problem $\operatorname{CSP}(\mathbf{B})$, in which, given a structure $\mathbf{A}$ of the same signature as $\mathbf{B}$, the goal is to decide whether or not there is a homomorphism from $\mathbf{A}$ to $\mathbf{B}$. For a class of similar algebras $\mathcal{A}=\left\{\mathbb{A}_{i} \mid i \in I\right\}$ for some set $I$ an instance of $\operatorname{CSP}(\mathcal{A})$ is a triple $(V, \delta, \mathcal{C})$, where $V$ is a set of variables; $\delta: V \rightarrow \mathcal{A}$ is a type function that associates every variable with a domain in $\mathcal{A}$. Finally, $\mathcal{C}$ is a set of constraints, i.e. pairs $\langle\mathbf{s}, R\rangle$, where $\mathbf{s}=\left(v_{1}, \ldots, v_{k}\right)$ is a tuple of variables from $V$, the constraint scope, and $R \in \operatorname{lnv}(\mathcal{A})$, a subset of $\mathbb{A}_{\delta\left(v_{1}\right)} \times \cdots \times \mathbb{A}_{\delta\left(v_{k}\right)}$, the constraint relation. The goal is to find a solution, that is a mapping $\varphi: V \rightarrow \bigcup \mathcal{A}$ such that $\varphi(v) \in \mathbb{A}_{\delta(v)}$ and for every constraint $\langle\mathbf{s}, R\rangle, \varphi(\mathbf{s}) \in R$. It is easy to see that if $\mathcal{A}$ is a class containing just one algebra $\mathbb{A}$, then $\operatorname{CSP}(\mathcal{A})$ can be viewed as the union of $\operatorname{CSP}(\mathbf{A})$ for all relational structures $\mathbf{A}$ invariant under the operations of $\mathbb{A}$. To simplify the notation we always write $\mathbb{A}_{v}$ rather than $\mathbb{A}_{\delta(v)}$, because the mapping $\delta$ is always clear from the context. This also allows us to simplify the notation for instances to $\mathcal{P}=(V, \mathcal{C})$. To allow for transformations of CSP described below we assume that $\mathcal{A}$ is closed under taking subalgebras and quotient algebras.

The set of solutions of a CSP instance $\mathcal{P}=(V, \mathcal{C})$ will be denoted by $\mathcal{S}_{\mathcal{P}}$, or just $\mathcal{S}$ if $\mathcal{P}$ is clear from the context. For $W \subseteq V$ by $\mathcal{P}_{W}$ we denote the restriction of $\mathcal{P}$ onto $W$, that is, the instance $\left(W, \mathcal{C}_{W}\right)$, where for each $C=\langle\mathbf{s}, R\rangle \in \mathcal{C}$, the set $\mathcal{C}_{W}$ includes the constraint $C_{W}=\left\langle\mathbf{s} \cap W, \operatorname{pr}_{\mathrm{s} \cap W} R\right\rangle$. The set of solutions of $\mathcal{P}_{W}$ will be denoted by $\mathcal{S}_{W}$. For $v \in V$ and a subalgebra $\mathbb{B}$ of $\mathbb{A}_{v}$ by $\mathcal{P}_{(v, \mathbb{B})}$ we denote the instance $\mathcal{P}$ with an extra constraint $\langle\{v\}, \mathbb{B}\rangle$; note that this is essentially equivalent to reducing the domain of $v$, and this is how we usually consider this construction. For $C=\langle\mathbf{s}, R\rangle \in \mathcal{C}$ let $R^{\prime}$ be a subalgebra of $R$ and $C^{\prime}=\left\langle\mathbf{s}, R^{\prime}\right\rangle$. The instance obtained from $\mathcal{P}$ replacing $C$ with $C^{\prime}$ is denoted by $\mathcal{P}_{C \rightarrow C^{\prime}}$. The transformation of $\mathcal{P}$ by reducing the domain of a variable $v \in V$ or reducing a constraint $C \in \mathcal{C}$, that is, transforming $\mathcal{P}$ into $\mathcal{P}_{(v, \mathbb{B})}$ or $\mathcal{P}_{C \rightarrow C^{\prime}}$ in such a way that the new instance has a solution if and only if $\mathcal{P}$ does, will be called tightening of $\mathcal{P}$. Let $\alpha_{v}$ be a congruence of $\mathbb{A}_{v}$ for each $v \in V$. By $\mathcal{P} / \bar{\alpha}$ we denote the instance $\left(V, \mathcal{C}^{\bar{\alpha}}\right)$ constructed as follows: the domain of $v \in V$ is $\mathbb{A}_{v} / \alpha_{v}$; for every constraint $C=\langle\mathbf{s}, R\rangle \in \mathcal{C}$, the set $\mathcal{C}^{\bar{\alpha}}$ includes the constraint $\left\langle\mathbf{s}, R / \bar{\alpha}_{\mathbf{s}}\right\rangle$. Note that if $\mathcal{A}$ is closed under taking subalgebras and quotient algebras, then applying a transformation of one of these kinds to an instance from $\operatorname{CSP}(\mathcal{A})$ results again in an instance from $\operatorname{CSP}(\mathcal{A})$.

Instance $\mathcal{P}$ is said to be minimal (or globally minimal) if for every $C=\langle\mathbf{s}, R\rangle \in$ 
$\mathcal{C}$ and every a $\in R$ there is a solution $\varphi \in \mathcal{S}$ such that $\varphi(\mathbf{s})=\mathbf{a}$. Instance $\mathcal{P}$ is said to be 1-minimal if for every $v \in V$ and every constraint $C=\langle\mathbf{s}, R\rangle \in \mathcal{C}$ such that $v \in \mathbf{s}, \operatorname{pr}_{v} R=\mathcal{S}_{v}$. Instance $\mathcal{P}$ is said to be (2,3)-consistent if it has a (2,3)-strategy, that is, a collection of relations $R^{X}, X \subseteq V,|X|=2$ satisfying the following conditions:

- for every $X \subseteq V$ with $|X| \leq 2$ and any constraint $C=\langle\mathrm{s}, R\rangle, \operatorname{pr}_{\mathrm{s} \cap X} R^{X} \subseteq$ $\operatorname{pr}_{\mathbf{s} \cap X} R$

- for every $X=\{u, v\} \subseteq V$, any $w \in V-X$ and any $(a, b) \in R^{X}$, there is $c \in \mathbb{A}_{w}$ such that $(a, c) \in R^{\{u, w\}}$ and $(b, c) \in R^{\{v, w\}}$.

We will always assume that a $(2,3)$-consistent instance has a constraint $C^{X}=$ $\left\langle X, \mathcal{S}_{X}\right\rangle$ for every $X \subseteq V,|X|=2$. Then clearly $R^{X} \subseteq \mathcal{S}_{X}$. Let the collection of relations $R^{X}$ be denoted by $\mathcal{R}$. A tuple a whose entries are indexed with elements of $W \subseteq V$ such that $\operatorname{pr}_{X} \mathbf{a} \in R^{X}$ for any $X \subseteq W,|X|=2$, will be called $\mathcal{R}$-compatible. If a (2,3)-consistent instance $\mathcal{P}$ with a (2,3)-strategy $\mathcal{R}$ satisfies the additional condition

- for every constraint $C=\langle\mathbf{s}, R\rangle$ of $\mathcal{P}$ every tuple $\mathbf{a} \in R$ is $\mathcal{R}$-compatible,

it is called (2,3)-minimal. Any instance can be transformed to a 1-minimal, (2,3)consistent, or (2,3)-minimal instance in polynomial time using the standard constraint propagation algorithms (see, e.g. [32] or [27]). These algorithms tighten the instance.

\subsection{Minimal sets and polynomials}

We will use the following basic facts from the tame congruence theory [39], often without further notice.

Let $\mathbb{A}$ be a finite algebra and $\alpha, \beta \in \operatorname{Con}(\mathbb{A})$ with $\alpha \prec \beta$. An $(\alpha, \beta)$-minimal set is a set minimal with respect to inclusion among the sets of the form $f(\mathbb{A})$, where $f \in \operatorname{Pol}_{1}(\mathbb{A})$ is such that $f(\beta) \nsubseteq \alpha$. Sets $B, C$ are said to be polynomially isomorphic in $\mathbb{A}$ if there are $f, g \in \operatorname{Pol}_{1}(\mathbb{A})$ such that $f(B)=C, g(C)=B$, and $f \circ g, g \circ f$ are identity mappings on $C$ and $B$, respectively.

Lemma 14 (Theorem 2.8, [39]) Let $\alpha, \beta \in \operatorname{Con}(\mathbb{A}), \alpha \prec \beta$. Then the following hold.

(1) Any $(\alpha, \beta)$-minimal sets $U, V$ are polynomially isomorphic.

(2) For any $(\alpha, \beta)$-minimal set $U$ and any $f \in \operatorname{Pol}_{1}(\mathbb{A})$, if $f\left(\left.\beta\right|_{U}\right) \nsubseteq \alpha$ then $f(U)$ is an $(\alpha, \beta)$-minimal set, $U$ and $f(U)$ are polynomially isomorphic, and $f$ witnesses this fact.

(3) For any $(\alpha, \beta)$-minimal set $U$ there is $f \in \operatorname{Pol}_{1}(\mathbb{A})$ such that $f(\mathbb{A})=U$, $f(\beta) \nsubseteq \alpha$, and $f$ is idempotent, in particular, $f$ is the identity mapping on $U$. 
(4) For any $(a, b) \in \beta-\alpha$ and an $(\alpha, \beta)$-minimal set $U$ there is $f \in \operatorname{Pol}_{1}(\mathbb{A})$ such that $f(\mathbb{A})=U$ and $\left.(f(a), f(b)) \in \beta\right|_{U}-\left.\alpha\right|_{U}$. Moreover, $f$ can be chosen to satisfy the conditions of item (3).

(5) For any $(\alpha, \beta)$-minimal set $U, \beta$ is the transitive closure of

$$
\alpha \cup\left\{(f(a), f(b))|(a, b) \in \beta|_{U}, f \in \operatorname{Pol}_{1}(\mathbb{A})\right\} .
$$

In fact, as $\alpha \prec \beta$ this claim can be strengthen to the following. For any $(a, b) \in$ $\beta-\alpha, \beta$ is the transitive closure of

$$
\alpha \cup\left\{(f(a), f(b)) \mid f \in \mathrm{Pol}_{1}(\mathbb{A})\right\} .
$$

(6) For any $f \in \operatorname{Pol}_{1}(\mathbb{A})$ such that $f(\beta) \nsubseteq \alpha$ there is an $(\alpha, \beta)$-minimal set $U$ such that $f$ witnesses that $U$ and $f(U)$ are polynomially isomorphic.

For an $(\alpha, \beta)$-minimal set $U$ and a $\beta$-block $B$ such that $\left.\beta\right|_{U \cap B} \neq\left.\alpha\right|_{U \cap B}$, the set $U \cap B$ is said to be an $(\alpha, \beta)$-trace. A 2-element set $\{a, b\} \subseteq U \cap B$ such that $(a, b) \in \beta-\alpha$, is called an $(\alpha, \beta)$-subtrace. The union $Q$ of the traces from $U$ is called the body of $U$, and $U-Q$ is called the tail of $U$. Depending on the structure of its minimal sets the interval $(\alpha, \beta)$ can be of one of the five types, 1-5. Since we assume the tractability conditions of the Dichotomy Conjecture, type $\mathbf{1}$ does not occur in algebras we deal with.

Lemma 15 (Section 4 of [39]) Let $\alpha, \beta \in \operatorname{Con}(\mathbb{A})$ and $\alpha \prec \beta$. Then the following hold.

(1) If typ $(\alpha, \beta)=\mathbf{2}$ then every $(\alpha, \beta)$-trace is polynomially equivalent to a 1 dimensional vector space.

(2) If $\operatorname{typ}(\alpha, \beta) \in\{\mathbf{3}, \mathbf{4}, \mathbf{5}\}$ then every $(\alpha, \beta)$-minimal set $U$ contains exactly one trace $T$, and if $\operatorname{typ}(\alpha, \beta) \in\{\mathbf{3}, \mathbf{4}\}$, T contains only 2 elements. Also, $T / \alpha$ is polynomially equivalent to a Boolean algebra, 2-element lattice, or 2-element semilattice, respectively.

Intervals $(\alpha, \beta),(\gamma, \delta), \alpha, \beta, \gamma, \delta \in \operatorname{Con}(\mathbb{A})$ and $\alpha \prec \beta, \gamma \prec \delta$ are said to be perspective if $\beta=\alpha \vee \delta, \gamma=\alpha \wedge \delta$, or $\delta=\beta \vee \gamma, \alpha=\beta \wedge \gamma$.

Lemma 16 (Lemma 6.2, [39]) Let $\alpha, \beta, \gamma, \delta \in \operatorname{Con}(\mathbb{A})$ be such that $\alpha \prec \beta, \gamma \prec$ $\delta$ and intervals $(\alpha, \beta),(\gamma, \delta)$ are perspective. Then $\operatorname{typ}(\alpha, \beta)=\operatorname{typ}(\gamma, \delta)$ and a set $U$ is $(\alpha, \beta)$-minimal if and only if it is $(\gamma, \delta)$-minimal.

We will also use polynomials that behave on a minimal set in a particular way. 
Lemma 17 (Lemmas 4.16, 4.17, [39]) Let $\alpha, \beta \in \operatorname{Con}(\mathbb{A}), \alpha \prec \beta$, and $\operatorname{typ}(\alpha, \beta) \in$ $\{\mathbf{3}, \mathbf{4}, \mathbf{5}\}$. Let $U$ be an $(\alpha, \beta)$-minimal set and $T$ its only trace. Then there is element $1 \in T$ and a binary polynomial $g$ of $\mathbb{A}$ such that

(1) $(1, a) \notin \alpha$ for any $a \in U-\{1\}$;

(2) for all $a \in U-\{1\}$, the algebra $(\{a, 1\}, g)$ is a semilattice with neutral element 1 , that is, $g(1,1)=1$ and $g(1, a)=g(a, 1)=g(a, a)=a$.

(3) for any $a \in U-\{1\}$ and any $b \in T-\{1\}, g(a, b) \stackrel{\alpha}{\equiv} g(b, a) \stackrel{\alpha}{\equiv} a$;

(4) for all $a, b \in U, g(a, g(a, b))=g(a, b)$.

Polynomial $g$ is said to be a pseudo-meet on $U$.

\subsection{Coloured graphs}

In [16, 28] we introduced a local approach to the structure of finite algebras. As we use this approach throughout the paper, we present it here in some details, see also [20]. For the sake of the definitions below we slightly abuse terminology and by a module mean the full idempotent reduct of a module.

For an algebra $\mathbb{A}$ graph $\mathcal{G}(\mathbb{A})$ is defined as follows. The vertex set is the universe $A$ of $\mathbb{A}$. A pair $a b$ of vertices is an $e d g e$ if and only if there exists a congruence $\theta$ of $\operatorname{Sg}(a, b)$, other than the full congruence and a term operation $f$ of $\mathbb{A}$ such that either $\operatorname{Sg}(a, b) /{ }_{\theta}$ is a module and $f$ is an affine operation on it, or $f$ is a semilattice operation on $\left\{a^{\theta}, b^{\theta}\right\}$, or $f$ is a majority operation on $\left\{a^{\theta}, b^{\theta}\right\}$. (Note that we use the same operation symbol in this case.) Usually, $\theta$ is chosen to be a maximal congruence of $\mathrm{Sg}(a, b)$.

If there are a congruence $\theta$ and a term operation $f$ of $\mathbb{A}$ such that $f$ is a semilattice operation on $\left\{a^{\theta}, b^{\theta}\right\}$ then $a b$ is said to have the semilattice type. An edge $a b$ is of majority type if there are a congruence $\theta$ and a term operation $f$ such that $f$ is a majority operation on $\left\{a^{\theta}, b^{\theta}\right\}$ and there is no semilattice term operation on $\left\{a^{\theta}, b^{\theta}\right\}$. Finally, $a b$ has the affine type if there are $\theta$ and $f$ such that $f$ is an affine operation on $\operatorname{Sg}(a, b) / \theta$ and $\operatorname{Sg}(a, b) / \theta$ is a module; in particular it implies that there is no semilattice or majority operation on $\left\{a^{\theta}, b^{\theta}\right\}$. In all cases we say that congruence $\theta$ witnesses the type of edge $a b$. Observe that a pair $a b$ can still be an edge of more than one type as witnessed by different congruences, although this has consequences in this paper.

Omitting type $\mathbf{1}$ can be characterized as follows.

Theorem 18 ([16, 20]) An idempotent algebra $\mathbb{A}$ omits type 1 (that is, the variety generated by $\mathbb{A}$ omits type if and only if $\mathcal{G}(\mathbb{B})$ is connected for every subalgebra $\mathbb{B}$ of $\mathbb{A}$. 
Moreover, a finite class $\mathcal{A}$ of similar idempotent algebras closed under subalgebras and quotient algebras omit type $\mathbf{1}$ if and only if $\mathcal{G}(\mathbb{A})$ is connected for any $\mathbb{A} \in \mathcal{A}$.

For the sake of the dichotomy conjecture, it suffices to consider reducts of an algebra $\mathbb{A}$ omitting type $\mathbf{1}$, that is, algebras with the same universe but reduced set of term operations, as long as reducts also omit type 1. In particular, we are interested in reducts of $\mathbb{A}$, in which semilattice and majority edges are subalgebras.

Theorem $19([16,[20])$ Let $\mathbb{A}$ be an algebra such that $\mathcal{G}(\mathbb{B})$ is connected for all subalgebras of $\mathbb{B}$ of $\mathbb{A}$, and let $a b$ be an edge of $\mathcal{G}(\mathbb{A})$ of the semilattice or majority type witnessed by congruence $\theta$, and $R_{a b}=a^{\theta} \cup b^{\theta}$. Let also $F_{a b}$ denote set of term operations of $\mathbb{A}$ preserving $R_{a b}$, and $\mathbb{A}^{\prime}=\left(A, F_{a b}\right)$. Then $\mathcal{G}\left(\mathcal{B}^{\prime}\right)$ is connected for all subalgebras $\mathbb{B}^{\prime}$ of $\mathbb{A}^{\prime}$.

An algebra $\mathbb{A}$ such that $a^{\theta} \cup b^{\theta}$ is a subuniverse of $\mathbb{A}$ for every semilattice or majority edge $a b$ of $\mathbb{A}$ is called sm-smooth. If $\mathcal{A}$ is the class of all quotient algebras of subalgebras of an sm-smooth algebra $\mathbb{A}$, it is easy to see that that every $\mathbb{B} \in \mathcal{A}$ is sm-smooth. Although it is not needed in this paper, for any finite class $\mathcal{A}$ omitting type 1 there is a class $\mathcal{A}^{\prime}$ of sm-smooth algebras which are reducts of algebras from $\mathcal{A}$, and such that $\mathcal{A}^{\prime}$ omits type 1 , as well. In the rest of the paper all algebras are assumed to be sm-smooth.

The next statement uniformizes the operations witnessing the type of edges.

Theorem 20 ([16, 20]) Let $\mathcal{A}$ be a class of similar idempotent algebra closed under taking subalgebras and quotient algebras. There are term operations $f, g, h$ of $\mathcal{A}$ such that for any $\mathbb{A} \in \mathcal{A}$ and any $a, b \in \mathbb{A}$ operation $f$ is a semilattice operation on $\left\{a^{\theta}, b^{\theta}\right\}$ if $a b$ is a semilattice edge; $g$ is a majority operation on $\left\{a^{\theta}, b^{\theta}\right\}$ if $a b$ is a majority edge; $h$ is an affine operation on $\mathrm{Sg}(a, b) / \theta$ if ab is an affine edge, where $\theta$ witnesses the type of the edge. Moreover, $f, g, h$ can be chosen such that

(1) $f(x, f(x, y))=f(x, y)$ for all $x, y \in \mathbb{A}, \mathbb{A} \in \mathcal{A}$;

(2) $g(x, g(x, y, y), g(x, y, y))=g(x, y, y)$ for all $x, y \in \mathbb{A}, \mathbb{A} \in \mathcal{A}$;

(3) $h(h(x, y, y), y, y)=h(x, y, y)$ for all $x, y \in \mathbb{A}, \mathbb{A} \in \mathcal{A}$.

There is a term operation $t$ such that for any affine edge ab and a majority, edge $c d$ witnessed by congruences $\eta$ and $\theta$, respectively, $t(a, b) \stackrel{\eta}{=} a$ and $t(c, d) \stackrel{\theta}{\equiv} d$.

Unlike majority and affine operations, for a semilattice edge $a b$ and a congruence $\theta$ of $\operatorname{Sg}(a, b)$ witnessing that, there can be semilattice operations acting differently on $\left\{a^{\theta}, b^{\theta}\right\}$, which corresponds to the two possible orientations of $a b$. In 
every such case by fixing operation $f$ from Theorem 20 we effectively choose one of the two orientations. In this paper we do not really care about what orientation is preferable.

In [20] we introduced a stronger notion of edge. A pair $a b$ of elements of algebra $\mathbb{A}$ is called a thin semilattice edge if $a b$ is a semilattice edge, and the congruence witnessing that is the equality relation. In other words, $f(a, a)=a$ and $f(a, b)=f(b, a)=f(b, b)=b$. We denote the fact that $a b$ is a thin semilattice edge by $a \leq b$. Thin semilattice edges allow us to introduce a directed graph $\mathcal{G}_{s}(\mathbb{A})$, whose vertices are the elements of $\mathbb{A}$, and the arcs are the thin semilattice edges. We then can define semilattice-connected and strongly semilatticeconnected components of $\mathcal{G}_{s}(\mathbb{A})$. We will also use the natural order on the set of strongly semilattice-connected components of $\mathcal{G}_{s}(\mathbb{A})$ : for components $A, B$, we write $A \leq B$ if there is a directed path in $\mathcal{G}_{s}(\mathbb{A})$ connecting a vertex from $A$ with a vertex from $B$. Elements from the maximal strongly connected components (or simply maximal components) of $\mathcal{G}_{s}(\mathbb{A})$ are called maximal elements of $\mathbb{A}$ and the set of all such elements is denoted by $\max (\mathbb{A})$. A directed path in $\mathcal{G}_{s}(\mathbb{A})$ is called a semilattice path or s-path. If there is an s-path from $a$ to $b$ we write $a \sqsubseteq b$.

Proposition 21 ([16, 20]) Let $\mathcal{A}$ be a finite class of similar idempotent algebras closed under taking subalgebras and quotient algebras. There is a binary term operation $f$ of $\mathcal{A}$ such that $f$ is a semilattice operation on $\left\{a^{\theta}, b^{\theta}\right\}$ for every semilattice edge ab of any $\mathbb{A} \in \mathcal{A}$, where congruence $\theta$ witnesses the type of $a b$, and, for any $a, b \in \mathbb{A}$, either $a=f(a, b)$ or the pair $(a, f(a, b))$ is a thin semilattice edge of $\mathbb{A}$. Operation $f$ with this property will be denoted by a dot (think multiplication).

Let operations $g, h$ be as in Theorem 20, A pair $a b$ from $\mathbb{A} \in \mathcal{A}$ is called a thin majority edge if (a) it is a majority edge, let congruence $\theta$ witness this, (b) for any $c \in b^{\theta}, b \in \operatorname{Sg}(a, c)$, (c) $g(a, b, b)=b$, and (d) there exists a ternary term operation $g^{\prime}$ such that $g^{\prime}(a, b, b)=g^{\prime}(b, a, b)=g^{\prime}(b, b, a)=b$. Finally, a pair $a b$ is called a thin affine edge if (a) it is an affine edge, let congruence $\theta$ witness this, (b) for any $c \in b^{\theta}, b \in \operatorname{Sg}(a, c)$, (c) $h(b, a, a)=b$, (d) there exists a ternary term operation $h^{\prime}$ such that $h^{\prime}(b, a, a)=h^{\prime}(a, a, b)=b$, and (e) $a$ is maximal in $\operatorname{Sg}(a, b)$. Note that the operations $h, g$ from Theorem 20 do not have to be majority or affine operations on thin edges; thin edges do not have to be even closed under $g, h$. Thin edges of all types are oriented. We therefore can define yet another directed graph, $\mathcal{G}^{\prime}(\mathbb{A})$, in which the arcs are the thin edges of all types.

Lemma 22 ([20]) Let $\mathbb{A}$ be an algebra.

(1) Let $a b$ be a semilattice or majority edge in $\mathbb{A}$, and $\theta$ the congruence of $\operatorname{Sg}(a, b)$ witnessing that. Then there is $b^{\prime} \in b^{\theta}$ such that $a b^{\prime}$ is a thin semilattice or majority 
edge, respectively.

(2) Let $a b$ be an affine edge, and $\theta$ the congruence of $\mathrm{Sg}(a, b)$ witnessing that. Then there are $a^{\prime} \in a^{\theta}$ and $b^{\prime} \in b^{\theta}$ such that $a \sqsubseteq a^{\prime}$ in $a^{\theta}$ and $a^{\prime} b^{\prime}$ is a thin affine edge.

The following simple properties of thin edges will be useful. Note that a subdirect product of algebras (a relation) is also an algebra, and so edges and thin edges can be defined for relations as well.

Lemma 23 ([20]) (1) Let $\mathbb{A}$ be an algebra and $a b$ a thin edge. Then $a b$ is a thin edge in any subalgebra of $\mathbb{A}$ containing $a, b$, and $a^{\theta} b^{\theta}$ is a thin edge in $\mathbb{A} /{ }_{\theta}$ for any congruence $\theta$.

(2) Let $R$ be a subdirect product of $\mathbb{A}_{1}, \ldots, \mathbb{A}_{n}, I \subseteq[n]$, and $\mathbf{a b}$ a thin edge in $R$. Then $\operatorname{pr}_{I} \mathbf{a p r}_{I} \mathbf{b}$ is a thin edge in $\operatorname{pr}_{I} R$ of the same type as $\mathbf{a b}$.

We will need stronger versions of Lemmas 18 and 20 of [20]. Let $\mathcal{A}$ be a finite class of similar idempotent algebras closed under taking subalgebras and quotient algebras.

Lemma 24 (1) Let ab be a thin majority edge of algebra $\mathbb{A} \in \mathcal{A}$. There is a term operation $t_{a b}$ such that $t_{a b}(a, b)=b$ and $t_{a b}(c, d) \stackrel{\theta_{c d}}{=} c$ for all affine edges $c d$ of all $\mathbb{A}^{\prime} \in \mathcal{A}$, where the type of $c d$ is witnessed by congruence $\theta_{c d}$.

(2) Let ab be a thin affine edge of algebra $\mathbb{A} \in \mathcal{A}$. There is a term operation $h_{a b}$ such that $h_{a b}(a, a, b)=b$ and $h_{a b}(d, c, c) \stackrel{\theta_{c d}}{=} d$ for all affine edges $c d$ of all $\mathbb{A}^{\prime} \in \mathcal{A}$, where the type of $c d$ is witnessed by congruence $\theta_{c d}$. Moreover, $h_{a b}\left(x, c^{\prime}, d^{\prime}\right)$ is a permutation of $\mathrm{Sg}(c, d) / \theta_{c d}$ for any $c^{\prime}, d^{\prime} \in \operatorname{Sg}(c, d)$.

(3) Let $a b$ and $c d$ be thin edges in algebras $\mathbb{A}, \mathbb{A}^{\prime} \in \mathcal{A}$, respectively. If they have different types there is a binary term operation $p$ such that $p(a, b)=a, p(c, d)=d$. If both edges are affine then there is a term operation $h^{\prime}$ such that $h^{\prime}(a, a, b)=b$ and $h^{\prime}(d, c, c)=d$.

Proof: (1) Let $c_{1} d_{1}, \ldots, c_{\ell} d_{\ell}$ be a list of all affine edges of algebras in $\mathcal{A}$, $c_{i}, d_{i} \in \mathbb{A}_{i}$ and $\theta_{c_{i} d_{i}}$ the corresponding congruences. Set $\mathbf{c}=\left(c_{1}, \ldots, c_{\ell}\right), \mathbf{d}=$ $\left(d_{1}, \ldots, d_{\ell}\right)$. Let $R$ be the subalgebra of $\mathbb{A} \times \prod_{i=1}^{\ell} \mathbb{A}_{i}$ generated by $(a, \mathbf{c}),(b, \mathbf{d})$. Pair $a b$ is also a majority edge, let it be witnessed by a congruence $\theta$. By Theorem 20

$$
\left(\begin{array}{l}
b^{\prime} \\
\mathbf{c}^{\prime}
\end{array}\right)=t\left(\left(\begin{array}{l}
a \\
\mathbf{c}
\end{array}\right),\left(\begin{array}{l}
b \\
\mathbf{d}
\end{array}\right)\right) \in R
$$

where $b^{\prime} \in b^{\theta}$ and $\mathbf{c}^{\prime}[i] \stackrel{\theta_{c_{i} d_{i}}}{=} \mathbf{c}[i]$, as $t$ is the first projection on $\operatorname{Sg}\left(c_{i}, d_{i}\right) / \theta_{c_{i} d_{i}}$ and a second projection on $\operatorname{Sg}(a, b) /_{\theta}$. Then as $b \in \operatorname{Sg}\left(a, b^{\prime}\right)$, we get $\left(b, \mathbf{c}^{\prime \prime}\right) \in R$ for 
some $\mathbf{c}^{\prime \prime}$ such that $\mathbf{c}^{\prime \prime}[i] \stackrel{\theta_{c_{i_{i}} d_{i}}}{=} \mathbf{c}[i]$. This means there is a binary term operation $t_{a b}$ such that

$$
t_{a b}\left(\left(\begin{array}{l}
a \\
\mathbf{c}
\end{array}\right),\left(\begin{array}{l}
b \\
\mathbf{d}
\end{array}\right)\right)=\left(\begin{array}{c}
b \\
\mathbf{c}^{\prime \prime}
\end{array}\right)
$$

The result follows.

(2) We use the notation from item (1) except $a b$ now is a thin affine edge and $R$ is generated by $(a, \mathbf{d}),(a, \mathbf{c}),(b, \mathbf{c})$. By condition (a) of the definition of thin affine edges,

$$
\left(\begin{array}{l}
b^{\prime} \\
\mathbf{d}^{\prime}
\end{array}\right)=h\left(\left(\begin{array}{l}
a \\
\mathbf{d}
\end{array}\right),\left(\begin{array}{l}
a \\
\mathbf{c}
\end{array}\right),\left(\begin{array}{l}
b \\
\mathbf{c}
\end{array}\right)\right) \in R
$$

where $b^{\prime} \in b^{\theta}$ and $\mathbf{d}^{\prime}[i] \stackrel{\theta_{c_{i} d_{i}}}{=} \mathbf{d}[i]$, as $h$ is a Mal'tsev operation on $\operatorname{Sg}(a, b) /{ }_{\theta}$ and on $\operatorname{Sg}\left(c_{i}, d_{i}\right) / \theta_{c_{i} d_{i}}$. Then as $b \in \operatorname{Sg}\left(a, b^{\prime}\right)$, by condition (b) we get $\left(b, \mathbf{d}^{\prime \prime}\right) \in R$ for some $\mathbf{d}^{\prime \prime}$ such that $\mathbf{d}^{\prime \prime}[i] \stackrel{\theta_{c_{i} d_{i}}}{=} \mathbf{d}[i]$. The first result follows.

Let now $h_{a b}$ be the term operation we constructed and $c^{\prime}, d^{\prime} \in \operatorname{Sg}\left(c_{i}, d_{i}\right), i \in$ $[\ell]$. Since $\mathbb{B}=\operatorname{Sg}\left(c_{i}, d_{i}\right) / \theta_{c_{i} d_{i}}$ is a module, in particular, it is an Abelian algebra and $h_{a b}\left(x, c^{*}, c^{*}\right)=x$ for all $c^{*} \in \mathbb{B}$, the second result follows.

(3) Follows from [20], Lemmas 15,18,19,20.

\subsection{Maximality}

A directed path in $\mathcal{G}^{\prime}(\mathbb{A})$ is called an asm-path, if there is an asm-path from $a$ to $b$ we write $a \sqsubseteq_{a s m} b$. If all edges of this path are semilattice or affine, it is called an affine-semilattice path or an as-path, if there is an as-path from $a$ to $b$ we write $a \sqsubseteq a s b$. Similar to maximal components, we consider strongly connected components of $\mathcal{G}^{\prime}(\mathbb{A})$ with majority edges removed, and the natural partial order on such components. The maximal components will be called as-components, and the elements from as-components are called as-maximal; the set of all as-maximal elements of $\mathbb{A}$ is denoted by $\operatorname{amax}(\mathbb{A})$. If $a$ is an as-maximal element, the ascomponent containing $a$ is denoted as $(a)$. An alternative way to define as-maximal elements is as follows: $a$ is as-maximal if for every $b \in \mathbb{A}$ such that $a \sqsubseteq a s b$ it also holds that $b \sqsubseteq_{a s} a$. Finally, element $a \in \mathbb{A}$ is said to be universally maximal (or u-maximal for short) if for every $b \in \mathbb{A}$ such that $a \sqsubseteq$ asm $b$ it also holds that $b \sqsubseteq_{a s m} a$. The set of all u-maximal elements of $\mathbb{A}$ is denoted umax $(\mathbb{A})$.

Proposition 25 ([20]) Let $\mathbb{A}$ be an algebra. Then

(1) any $a, b \in \mathbb{A}$ are connected in $\mathcal{G}^{\prime}(\mathbb{A})$ with an undirected path;

(2) any $a, b \in \max (\mathbb{A})$ (or $a, b \in \operatorname{amax}(\mathbb{A})$, or $a, b \in \operatorname{umax}(\mathbb{A}))$ are connected in $\mathcal{G}^{\prime}(\mathbb{A})$ with a directed path. 
Proof: Item (2) is only proved in [20] for maximal and as-maximal elements; so we prove it here for $\mathrm{u}$-maximal elements as well. Let $a^{\prime}, b^{\prime} \in \mathbb{A}$ be maximal elements of $\mathbb{A}$ such that $a \sqsubseteq a^{\prime}$ and $b \sqsubseteq b^{\prime}$. Then by Proposition 25 for maximal elements $a^{\prime} \sqsubseteq_{a s m} b^{\prime}$, and, as $b$ is u-maximal, $b^{\prime} \sqsubseteq_{a s m} b$.

Since for every $a \in \mathbb{A}$ there is a maximal $a^{\prime} \in \mathbb{A}$ such that $a \sqsubseteq a^{\prime}$, Proposition 25 implies that there is only one u-maximal component. U-maximality has an additional useful property, it is somewhat hereditary, as it made precise in the following

Lemma 26 Let $\mathbb{B}$ be a subalgebra of $\mathbb{A}$ containing a u-maximal element of $\mathbb{A}$. Then every element u-maximal in $\mathbb{B}$ is also u-maximal in $\mathbb{A}$. In particular, if $\alpha$ is a congruence of $\mathbb{A}$ and $\mathbb{B}$ is a u-maximal $\alpha$-block, that is $\mathbb{B}$ is a u-maximal element in $\mathbb{A} / \alpha$, then $\operatorname{umax}(B) \subseteq \operatorname{umax}(\mathbb{A})$.

Proof: Let $a \in \mathbb{B}$ be an element u-maximal in $\mathbb{A}$, let $b \in \operatorname{umax}(\mathbb{B})$. For any $c \in \mathbb{A}$ with $b \sqsubseteq_{\text {asm }} c$ we also have $c \sqsubseteq_{\text {asm }} a$. Finally, since $b \in \operatorname{umax}(\mathbb{B})$ and $a \in \mathbb{B}$, we have $a \sqsubseteq a s m b$. For the second part of the lemma we need to find a umaximal element in $\mathbb{B}$. Let $b \in \operatorname{umax}(\mathbb{A})$. Then as $\mathbb{B}$ is u-maximal in $\mathbb{A} / \alpha$ applying Lemma 22 we get that there is $a^{\prime} \in \mathbb{B}$ such that $b \sqsubseteq_{a s m} a^{\prime}$. Clearly, $a^{\prime} \in \operatorname{umax}(\mathbb{A})$.

Let $\mathbb{A}$ be an algebra and $a \in \mathbb{A}$. By $\operatorname{Ft}_{\mathbb{A}}(a)$ we denote the set of elements $a$ is connected to (in terms of semilattice paths); similarly, by $\mathrm{Ft}_{\mathbb{A}}^{a s}(a)$ and $\mathrm{Ft}_{\mathbb{A}}^{a s m}(a)$ we denote the set of elements $a$ is as-connected and asm-connected to. Also, $\mathrm{Ft}_{\mathbb{A}}(C)=\bigcup_{a \in C} \mathrm{Ft}_{\mathbb{A}}(a)\left(\mathrm{Ft}_{\mathbb{A}}^{a s}(C)=\bigcup_{a \in C} \mathrm{Ft}_{\mathbb{A}}^{a s}(a), \mathrm{Ft}_{\mathbb{A}}^{a s m}(C)=\bigcup_{a \in C} \mathrm{Ft}_{\mathbb{A}}^{a s m}(a)\right.$, respectively) for $C \subseteq \mathbb{A}$. Note that if $a$ is an as-maximal element then as $(a)=$ $\mathrm{Ft}_{\mathbb{A}}^{a s}(a)$, and $a \in \mathrm{Ft}_{\mathbb{A}}^{a s m}(b)$ for any $b \in \mathbb{A}$. We will need the following statements.

Lemma 27 (The Maximality Lemma,[21]) Let $R$ be a subdirect product of $\mathbb{A}_{1} \times$ $\cdots \times \mathbb{A}_{n}, I \subseteq[n]$.

(1) For any $\mathbf{a} \in R, \mathbf{b} \in \operatorname{pr}_{I} R$ with $\operatorname{pr}_{I} \mathbf{a} \leq \mathbf{b}$, there is $\mathbf{b}^{\prime} \in R$ such that $\mathbf{a} \leq \mathbf{b}^{\prime}$ and $\operatorname{pr}_{I} \mathbf{b}^{\prime}=\mathbf{b}$.

(2) For any $\mathbf{a} \in R, \mathbf{b} \in \operatorname{pr}_{I} R$ such that $\left(\operatorname{pr}_{I} \mathbf{a}\right) \mathbf{b}$ is a thin majority edge there is $\mathbf{b}^{\prime} \in R$ such that $\mathbf{a} \mathbf{b}^{\prime}$ is a thin majority edge, and $\operatorname{pr}_{I} \mathbf{b}^{\prime}=\mathbf{b}$.

(3) For any $\mathbf{a} \in R, \mathbf{b} \in \operatorname{pr}_{I} R$ such that $\left(\operatorname{pr}_{I} \mathbf{a}\right) \mathbf{b}$ is a thin affine edge there are $\mathbf{a}^{\prime}, \mathbf{b}^{\prime} \in R$ such that $\mathbf{a} \sqsubseteq \mathbf{a}^{\prime}, \mathbf{a}^{\prime} \mathbf{b}^{\prime}$ is a thin affine edge, and $\operatorname{pr}_{I} \mathbf{a}^{\prime}=\operatorname{pr}_{I} \mathbf{a}$, $\operatorname{pr}_{I} \mathbf{b}^{\prime}=\mathbf{b}$.

(4) For any $\mathbf{a} \in R$, and an s-path (as-path, asm-path) $\mathbf{b}_{1}, \ldots, \mathbf{b}_{k} \in \operatorname{pr}_{I} R$ with $\operatorname{pr}_{I} \mathbf{a}=\mathbf{b}_{1}$, there is an s-path (as-path,asm-path, respectively) $\mathbf{b}_{1}^{\prime}, \ldots, \mathbf{b}_{\ell}^{\prime} \in R$ such that $\operatorname{pr}_{I} \mathbf{b}_{\ell}^{\prime}=\mathbf{b}_{\ell}$. 
(5) For any $\mathbf{b} \in \max \left(\operatorname{pr}_{I} R\right)\left(\mathbf{b} \in \operatorname{amax}\left(\operatorname{pr}_{I} R\right), \mathbf{b} \in \operatorname{umax}\left(\operatorname{pr}_{I} R\right)\right)$ there is $\mathbf{b}^{\prime} \in \max (R)$ ( $\mathbf{b}^{\prime} \in \operatorname{amax}(R), \mathbf{b}^{\prime} \in \operatorname{umax}(R)$, respectively), such that $\operatorname{pr}_{I} \mathbf{b}^{\prime}=$ b. In particular, $\operatorname{pr}_{[n]-I} \mathbf{b}^{\prime} \in \max \left(\operatorname{pr}_{[n]-I} R\right)\left(\operatorname{pr}_{[n]-I} \mathbf{b}^{\prime} \in \operatorname{amax}\left(\operatorname{pr}_{[n]-I} R\right)\right.$, $\operatorname{pr}_{[n]-I} \mathbf{b}^{\prime} \in \operatorname{umax}\left(\operatorname{pr}_{[n]-I} R\right)$, respectively).

Proof: Items (1) and (3) are proved in [21], and items of (4) and (5) are only proved for s- and as-paths, and, respectively, for maximal and as-maximal elements. Items (4) and (5) for asm-paths and u-maximal elements follow from (1)(3).

(2) Observe that it suffices to consider binary relations $R$. Indeed, $R$ can be viewed as a subdirect product of $\operatorname{pr}_{I} R \times \operatorname{pr}_{[n]-I} R$. So, suppose $n=2$ and $I=\{1\}$. We have $\mathbf{a}=\left(a_{1}, a_{2}\right)$ and $\mathbf{b}=b_{1}$. Let $\theta$ be a maximal congruence of $\operatorname{Sg}\left(a_{1}, b_{1}\right)$ witnessing that $a_{1} b_{1}$ is an majority edge. Choose $\mathbf{b}^{\prime \prime}=\left(b_{1}^{\prime}, b_{2}\right) \in R$ such that $b_{1}^{\prime} \in b_{1}^{\theta}$ and $R^{\prime}=\operatorname{Sg}\left(\mathbf{a}, \mathbf{b}^{\prime \prime}\right)$ is minimal possible with this condition. It suffices to prove the lemma for $R^{\prime}$, since $b_{1} \in \operatorname{Sg}\left(a_{1}, b_{1}^{\prime}\right)$ and so $b_{1} \in \operatorname{pr}_{1} R^{\prime}$, and $a_{1} b_{1}$ is still a thin majority edge. This means that $\left(b_{1}^{\prime}, b_{2}\right)$ can be chosen such that $b_{1}^{\prime}=b_{1}$. Also, by taking $\left(\begin{array}{l}b_{1} \\ b_{2}^{\prime}\end{array}\right)=g\left(\left(\begin{array}{l}a_{1} \\ a_{2}\end{array}\right),\left(\begin{array}{l}b_{1} \\ b_{2}\end{array}\right),\left(\begin{array}{l}b_{1} \\ b_{2}\end{array}\right)\right)$ we may assume by Theorem 20 that $g\left(a_{2}, b_{2}, b_{2}\right)=b_{2}$. As is easily seen, the pair $\left(a_{1}, a_{2}\right)\left(b_{1}, b_{2}\right)$ is a majority edge as witnessed by congruence $\theta^{\prime}=\theta \times \underline{1}_{\mathbb{A}_{2}^{\prime}}$ where $\mathbb{A}_{2}^{\prime}=\operatorname{Sg}\left(a_{2}, b_{2}\right)$. By the choice of $\mathbf{b}^{\prime \prime}$ the pair $\left(b_{1}, b_{2}\right)$ belongs to $\operatorname{Sg}\left(\left(a_{1}, a_{2}\right),\left(c_{1}, c_{2}\right)\right)$ for any $\left(c_{1}, c_{2}\right) \in\left(b_{1}, b_{2}\right)^{\theta^{\prime}}$, and it only remains to prove condition (d) of the definition of thin majority edges.

Let $g^{\prime}$ be the operation from condition (d) for $a_{1} b_{1}$. Then

$$
g^{\prime}\left(\left(\begin{array}{c}
\left(a_{1}, a_{2}\right) \\
\left(b_{1}, b_{2}\right) \\
\left(b_{1}, b_{2}\right)
\end{array}\right),\left(\begin{array}{c}
\left(b_{1}, b_{2}\right) \\
\left(a_{1}, a_{2}\right) \\
\left(b_{1}, b_{2}\right)
\end{array}\right),\left(\begin{array}{c}
\left(b_{1}, b_{2}\right) \\
\left(b_{1}, b_{2}\right) \\
\left(a_{1}, a_{2}\right)
\end{array}\right)\right)=\left(\begin{array}{c}
\left(b_{1}, b_{2}^{\prime}\right) \\
\left(b_{1}, b_{2}^{\prime \prime}\right) \\
\left(b_{1}, b_{2}^{\prime \prime \prime}\right)
\end{array}\right) .
$$

Since $\left(b_{1}, b_{2}\right) \in \operatorname{Sg}\left(\left(a_{1}, a_{2}\right),\left(b_{1}, b_{2}^{\prime}\right)\right)$ by the choice of $\left(b_{1}, b_{2}\right)$, there is a term operation $r_{1}$ such that

$$
r_{1}\left(\left(\begin{array}{c}
\left(a_{1}, a_{2}\right) \\
\left(b_{1}, b_{2}\right) \\
\left(b_{1}, b_{2}\right)
\end{array}\right),\left(\begin{array}{c}
\left(b_{1}, b_{2}^{\prime}\right) \\
\left(b_{1}, b_{2}^{\prime \prime}\right) \\
\left(b_{1}, b_{2}^{\prime \prime \prime}\right)
\end{array}\right)\right)=\left(\begin{array}{c}
\left(b_{1}, b_{2}\right) \\
\left(b_{1}, b_{2}^{*}\right) \\
\left(b_{1}, b_{2}^{* *}\right)
\end{array}\right) .
$$

Repeating this for the second and third coordinate positions by finding $r_{2}, r_{3}$ with

$$
\begin{aligned}
& r_{2}\left(\left(\begin{array}{c}
\left(b_{1}, b_{2}\right) \\
\left(a_{1}, a_{2}\right) \\
\left(b_{1}, b_{2}\right)
\end{array}\right),\left(\begin{array}{c}
\left(b_{1}, b_{2}\right) \\
\left(b_{1}, b_{2}^{*}\right) \\
\left(b_{1}, b_{2}^{* *}\right)
\end{array}\right)\right)=\left(\begin{array}{c}
\left(b_{1}, b_{2}\right) \\
\left(b_{1}, b_{2}\right) \\
\left(b_{1}, b_{2}^{\dagger}\right)
\end{array}\right), \\
& r_{3}\left(\left(\begin{array}{c}
\left(b_{1}, b_{2}\right) \\
\left(b_{1}, b_{2}\right) \\
\left(a_{1}, a_{2}\right)
\end{array}\right),\left(\begin{array}{c}
\left(b_{1}, b_{2}\right) \\
\left(b_{1}, b_{2}\right) \\
\left(b_{1}, b_{2}^{\dagger}\right)
\end{array}\right)\right)=\left(\begin{array}{c}
\left(b_{1}, b_{2}\right) \\
\left(b_{1}, b_{2}\right) \\
\left(b_{1}, b_{2}\right)
\end{array}\right),
\end{aligned}
$$


we obtain a ternary operation $g^{\prime \prime}$ such that

$$
\begin{aligned}
g^{\prime \prime}\left(\left(\begin{array}{l}
a_{1} \\
a_{2}
\end{array}\right),\left(\begin{array}{l}
b_{1} \\
b_{2}
\end{array}\right),\left(\begin{array}{l}
b_{1} \\
b_{2}
\end{array}\right)\right) & =g^{\prime \prime}\left(\left(\begin{array}{l}
b_{1} \\
b_{2}
\end{array}\right),\left(\begin{array}{l}
a_{1} \\
a_{2}
\end{array}\right),\left(\begin{array}{l}
b_{1} \\
b_{2}
\end{array}\right)\right) \\
& =g^{\prime \prime}\left(\left(\begin{array}{l}
b_{1} \\
b_{2}
\end{array}\right),\left(\begin{array}{l}
b_{1} \\
b_{2}
\end{array}\right)\left(\begin{array}{l}
a_{1} \\
a_{2}
\end{array}\right)\right)=\left(\begin{array}{l}
b_{1} \\
b_{2}
\end{array}\right),
\end{aligned}
$$

confirming property (d).

The following lemma considers a special case of as-components in subdirect products, and is straightforward.

Lemma 28 Let $R$ be a subdirect product of $\mathbb{A}_{1} \times \mathbb{A}_{2}, B, C$ as components of $\mathbb{A}_{1}, \mathbb{A}_{2}$, respectively, and $B \times C \subseteq R$. Then $B \times C$ is an as-component of $R$.

We complete this section with an auxiliary statement that will be needed later.

Lemma 29 Let $\alpha \prec \beta, \alpha, \beta \in \operatorname{Con}(\mathbb{A})$, let $B$ be a $\beta$-block and $\operatorname{typ}(\alpha, \beta)=\mathbf{2}$. Then $B / \alpha$ is term equivalent to a module. In particular, every pair of elements of $B / \alpha$ is a thin affine edge in $\mathbb{A} / \alpha$.

Proof: As $\mathbb{A}$ is an idempotent algebra that generates a variety omitting type $\mathbf{1}$, and $(\alpha, \beta)$ is a simple interval in $\operatorname{Con}(\mathbb{A})$ of type 2 , by Theorem 7.11 of [39] there is a term operation of $\mathbb{A}$ that is Mal'tsev on $B / \alpha$. Since $\beta$ is Abelian on $B / \alpha$, we get the result.

\subsection{Quasi-decomposition and quasi-majority}

We make use of the property of quasi-2-decomposability proved in [21].

Theorem 30 (The 2-Decomposition Theorem, [21]) If $R$ is an $n$-ary relation, $X \subseteq$ $[n]$, tuple a is such that $\operatorname{pr}_{J} \mathbf{a} \in \operatorname{pr}_{J} R$ for any $J \subseteq[n],|J|=2$, and $\operatorname{pr}_{X} \mathbf{a} \in$ $\operatorname{amax}\left(\operatorname{pr}_{X} R\right)$, there is a tuple $\mathbf{b} \in R$ with $\operatorname{pr}_{J} \mathbf{b} \in \mathrm{Ft}_{\operatorname{pr}_{J} R}^{\text {as }}\left(\operatorname{pr}_{J} \mathbf{a}\right)$ for any $J \subseteq[n]$, $|J|=2$, and $\operatorname{pr}_{X} \mathbf{b}=\operatorname{pr}_{X} \mathbf{a}$.

One useful implication of the 2-Decomposition Theorem 30 is the existence of term operation resembling a majority function. We state this theorem for finite classes of algebras rather than a single algebra, because it concerns as-components that in subalgebras of products may have complicated structure.

Theorem 31 Let $\mathcal{A}$ be a finite class of finite similar sm-smooth algebras omitting type 1. There is a term operation maj of $\mathcal{A}$ such that for any $\mathbb{A} \in \mathcal{A}$ and any $a, b \in \mathbb{A}, \operatorname{maj}(a, a, b), \operatorname{maj}(a, b, a), \operatorname{maj}(b, a, a) \in \mathrm{Ft}_{\mathbb{A}}^{a s}(a)$.

In particular, if a is as-maximal, then $\operatorname{maj}(a, a, b), \operatorname{maj}(a, b, a), \operatorname{maj}(b, a, a) b e-$ long to the as-component of $\mathbb{A}$ containing a. 
Proof: Let $\left\{a_{1}, b_{1}\right\}, \ldots,\left\{a_{n}, b_{n}\right\}$ be a list of all pairs of elements from algebras of $\mathcal{A}$, let $a_{i}, b_{i} \in \mathbb{A}_{i}$. Define relation $R$ to be a subdirect product of $\mathbb{A}_{1}^{3} \times \cdots \times \mathbb{A}_{n}^{3}$ generated by $\mathbf{a}_{1}, \mathbf{a}_{2}, \mathbf{a}_{3}$, where for every $i \in[n], \operatorname{pr}_{3 i-2,3 i-1,3 i} \mathbf{a}_{1}=$ $\left(a_{i}, a_{i}, b_{i}\right), \operatorname{pr}_{3 i-2,3 i-1,3 i} \mathbf{a}_{2}=\left(a_{i}, b_{i}, a_{i}\right), \operatorname{pr}_{3 i-2,3 i-1,3 i} \mathbf{a}_{3}=\left(b_{i}, a_{i}, a_{i}\right)$. In other words the triples $\left(\mathbf{a}_{1}[3 i-2], \mathbf{a}_{2}[3 i-2], \mathbf{a}_{3}[3 i-2]\right),\left(\mathbf{a}_{1}[3 i-1], \mathbf{a}_{2}[3 i-1], \mathbf{a}_{3}[3 i-\right.$ $1]),\left(\mathbf{a}_{1}[3 i], \mathbf{a}_{2}[3 i], \mathbf{a}_{3}[3 i]\right)$ have the form $\left(a_{i}, a_{i}, b_{i}\right),\left(a_{i}, b_{i}, a_{i}\right),\left(b_{i}, a_{i}, a_{i}\right)$, respectively. Therefore it suffices to show that $R$ contains a tuple $\mathbf{b}$ such that $a_{i} \sqsubseteq_{a s} \mathbf{b}[j]$, where $j \in\{3 i, 3 i-1,3 i-2\}$. However, since $\left(a_{i_{1}}, a_{i_{2}}\right) \in \operatorname{pr}_{j_{1} j_{2}} R$ for any $i_{1}, i_{2} \in[n]$ and $j_{1} \in\left\{3 i_{1}, 3 i_{1}-1,3 i_{1}-2\right\}, j_{2} \in\left\{3 i_{2}, 3 i_{2}-1,3 i_{2}-2\right\}$, this follows from the 2-Decomposition Theorem 30 .

A function maj satisfying the properties from Theorem 31 will be called a quasi-majority function.

\subsection{Rectangularity}

Let $R$ be a subdirect product of $\mathbb{A}_{1}, \mathbb{A}_{2}$. By $R[c], R^{-1}\left[c^{\prime}\right]$ for $c \in \mathbb{A}_{1}, c^{\prime} \in \mathbb{A}_{2}$ we denote the sets $\{b \mid(c, b) \in R\},\left\{a \mid\left(a, c^{\prime}\right) \in R\right\}$, respectively, and for $C \subseteq$ $\mathbb{A}_{1}, C^{\prime} \subseteq \mathbb{A}_{2}$ we use $R[C]=\bigcup_{c \in C} R[c], R^{-1}\left[C^{\prime}\right]=\bigcup_{c^{\prime} \in C^{\prime}} R^{-1}\left[c^{\prime}\right]$, respectively. Binary relations tol ${ }_{1}$, tol $_{2}$ on $\mathbb{A}_{1}, \mathbb{A}_{2}$ given by tol $(R)=\{(a, b) \mid R[a] \cap R[b] \neq \varnothing\}$ and tol $_{2}(R)=\left\{(a, b) \mid R^{-1}[a] \cap R^{-1}[b] \neq \varnothing\right\}$, respectively, are called link tolerances of $R$. They are tolerances of $\mathbb{A}_{1}, \mathbb{A}_{2}$, respectively, that is invariant reflexive and symmetric relations. The transitive closures $\mathrm{Ik}_{1}, \mathrm{lk}_{2}$ of $\mathrm{tol}_{1}(R), \mathrm{tol}_{2}(R)$ are called link congruences, and they are, indeed, congruences. Relation $R$ is said to be linked if the link congruences are full congruences.

Lemma 32 ([21]) Let $R$ be a subalgebra of $\mathbb{A}_{1} \times \mathbb{A}_{2}$ and let $a \in \mathbb{A}_{1}$ and $B=R[a]$. For any $b \in \mathbb{A}_{1}$ such that $a b$ is thin edge, and any $c \in R[b] \cap B, \mathrm{Ft}_{B}^{a s}(c) \subseteq R[b]$.

Proof: The case when $a \leq b$ or $a b$ is affine is considered in [21], so suppose that $a b$ is majority. Let $D=\mathrm{Ft}_{B}^{a s}(c) \cap R[b]$. Set $D$ is nonempty, as $c \in D$. If $D \neq \mathrm{Ft}_{B}^{a s}(c)$, there are $b_{1} \in D$ and $b_{2} \in \mathrm{Ft}_{B}^{a s}(c)-D$ such that $b_{1} b_{2}$ is a thin edge. By Lemma 24 (3) there is a term operation $p$ such that $p(a, b)=b$ and $p\left(b_{2}, b_{1}\right)=b_{2}$. Then $\left(\begin{array}{c}b \\ b_{2}\end{array}\right)=p\left(\left(\begin{array}{c}a \\ b_{2}\end{array}\right),\left(\begin{array}{c}b \\ b_{1}\end{array}\right)\right) \in R$. The result follows.

Proposition $33([21])$ Let $R \leq \mathbb{A}_{1} \times \mathbb{A}_{2}$ be a linked subdirect product and let $B_{1}, B_{2}$ be as-components of $\mathbb{A}_{1}, \mathbb{A}_{2}$, respectively, such that $R \cap\left(B_{1} \times B_{2}\right) \neq \varnothing$. Then $B_{1} \times B_{2} \subseteq R$.

Corollary 34 (The Rectangularity Corollary) Let $R$ be a subdirect product of $\mathbb{A}_{1}$ and $\mathbb{A}_{2}, \mathrm{Ik}_{1}, \mathrm{lk} \mathrm{k}_{2}$ the link congruences, and let $B_{1}, B_{2}$ be as-components of a 
$\mathrm{Ik}_{1}$-block and $a \mathrm{lk}_{2}$-block, respectively, such that $R \cap\left(B_{1} \times B_{2}\right) \neq \varnothing$. Then $B_{1} \times B_{2} \subseteq R$.

Proposition 35 Let $R$ be a subdirect product of $\mathbb{A}_{1}$ and $\mathbb{A}_{2}, \mathrm{Ik}_{1}, \mathrm{I} \mathrm{k}_{2}$ the link congruences, and let $B_{1}$ be an as-component of a $\mathrm{lk}_{1}$-block and $B_{2}^{\prime}=R\left[B_{1}\right]$; let $B_{2}=\operatorname{umax}\left(B_{2}^{\prime}\right)$. Then $B_{1} \times B_{2} \subseteq R$.

Proof: Let $B_{2}^{\prime}$ be a subset of a lk $\mathrm{k}_{2}$-block $C$. By the Maximality Lemma 27(5) $B_{2}^{\prime}$ contains an as-maximal element $a$ of $C$. By the Rectangularity Corollary 34 $B_{1} \times\{a\} \subseteq R$. It then suffices to show that $B_{1} \times \mathrm{Ft}_{B_{2}^{\prime}}^{a s m}(a) \subseteq R$.

Suppose for $D \subseteq \mathrm{Ft}_{B_{2}^{\prime}}^{a s m}(a)$ it holds $B_{1} \times D \subseteq R$. If $D \neq \mathrm{Ft}_{B_{2}^{\prime}}^{a s m}(a)$, there are $b_{1} \in D$ and $b_{2} \in \mathrm{Ft}_{B_{2}^{\prime}}^{a s m}(a)-D$ such that $b_{1} b_{2}$ is a thin edge. By Lemma 32 $B_{1} \times\left\{b_{2}\right\} \subseteq R$; the result follows.

We complete this section with a technical lemma that will be useful later.

Lemma 36 Let $\mathbb{A}$ be an algebra and $C$ its as-component such that $\mathbb{A}=\operatorname{Sg}(C)$, let $R=\mathbb{A} \times \mathbb{A}=\operatorname{Sg}(C \times C)$, and let $\beta$ be a congruence of $R$. Then for some $a, b \in C, a \neq b$ the pair $(a, b)$ is as-maximal in a $\beta$-block.

\section{Proof:}

We start with a general claim.

Claim. If $\beta, \gamma \in \operatorname{Con}(R)$ are such that $\beta \vee \gamma=\underline{1}_{R}$, then $\left.\beta\right|_{C^{2}} \circ \gamma_{C^{2}}=$ $\left.\left.\gamma\right|_{C^{2}} \circ \beta\right|_{C^{2}}=C^{2} \times C^{2}$.

Let $R_{1} \subseteq R / \beta \times R, R_{2} \subseteq R / \gamma \times R$ be given by

$$
R_{1}=\left\{\left(a^{\beta}, a\right) \mid a \in R\right\}, \quad R_{2}=\left\{\left(a^{\gamma}, a\right) \mid a \in R\right\} .
$$

Consider a subdirect product of $R / \beta \times R / \gamma$ defined as follows

$$
Q(x, y)=\exists z \in R \quad R_{1}(x, z) \wedge R_{2}(y, z) .
$$

As is easily seen, for a $\beta$-block $B$ and a $\gamma$-block $D,(B, D) \in Q$ if and only if $B \cap D \neq \varnothing$. As $\beta \vee \gamma=\underline{1}_{R}$, relation $Q$ is linked. Since $C^{2} / \beta$ is an ascomponent of $R /{ }_{\beta}$ and $C^{2} / \gamma$ is an as-component of $R / \gamma$, Proposition 33 implies that $C^{2} /{ }_{\beta} \times C^{2} / \gamma \subseteq Q$. Therefore for any $\beta$ - and $\gamma$-blocks $B, D$ such that $B \cap C^{2} \neq$ $\varnothing, D \cap B^{2} \neq \varnothing$ we have $B \cap D \neq \varnothing$. Using as-connectivity and Lemma 22 it can also be inferred that $B \cap D \cap C^{2} \neq \varnothing$. The result follows.

Let $\alpha$ be a maximal congruence of $\mathbb{A}$ and $\gamma_{1}=\alpha \times \underline{1}_{\mathbb{A}}, \gamma_{2}=\underline{1}_{\mathbb{A}} \times \alpha$. As is easily seen, $\gamma_{1}, \gamma_{2}$ are maximal congruences of $R$. There are two cases.

CASE 1. $\beta \vee\left(\gamma_{1} \wedge \gamma_{2}\right)=\underline{1}_{R}$. 
By the Claim for any $\alpha$-blocks $B_{1}, B_{2}$ such that $B_{1} \cap C, B_{2} \cap C \neq \varnothing$ we also have $B \cap\left(B_{1} \times B_{2}\right) \cap C^{2} \neq \varnothing$. Also, using Lemma 22 $B \cap\left(B_{1} \times B_{2}\right) \cap C^{2}$ contains a pair as-maximal in $B$. Choosing $B_{1} \neq B_{2}$ we get the result.

CASE 2. $\beta \vee\left(\gamma_{1} \wedge \gamma_{2}\right) \neq \underline{1}_{R}$.

In this case consider $\mathbb{A}^{\prime}=\mathbb{A} / \alpha, R^{\prime}=R / \alpha \times \alpha, \beta^{\prime}=\beta / \alpha$; note that $\mathbb{A}^{\prime}$ is a simple idempotent algebra, and as $R=\mathbb{A} \times \mathbb{A}$, we have $R^{\prime}=\mathbb{A}^{\prime} \times \mathbb{A}^{\prime}$. By [44] either $\mathbb{A}^{\prime}$ has an absorbing element $a$, that is, $f\left(a_{1}, \ldots, a_{k}\right)=a$ for any term operation $f$ of $\mathbb{A}^{\prime}$, whenever for some essential variable $x_{i}$ of $f, a_{i}=a$, or $\mathbb{A}^{\prime}$ is a module, or the only nontrivial congruences of $\mathbb{A}^{\prime}$ are $\gamma_{1}^{\prime}=\gamma_{1} / \alpha \times \alpha, \gamma_{2}^{\prime}=\gamma_{2} / \alpha \times \alpha$. Since $C$ is a nontrivial as-component, the first option is impossible. If $\mathbb{A}^{\prime}$ is a simple module, the only congruence that is different from $\gamma_{1}^{\prime}, \gamma_{2}^{\prime}$ is the skew congruence with $\Delta=\left\{(a, a) \mid a \in \mathbb{A}^{\prime}\right\}$ as a congruence block. If $\beta$ is the skew congruence, let $D$ be a $\beta \vee\left(\gamma_{1} \wedge \gamma_{2}\right)$-block different from $\Delta$ and $B \subseteq D$ a $\beta$-block. Then for any as-maximal pair $(a, b) \in B$ we have $a \neq b$.

So, suppose $\beta \leq \gamma_{1}$. If $\beta \leq \gamma_{1} \wedge \gamma_{2}$, choose a $\gamma_{1} \wedge \gamma_{2}$-block $B_{1} \times B_{2}$ such that $B_{1} \neq B_{2}$; clearly $B_{1}, B_{2}$ are $\alpha$-blocks. Then for any $\beta$-block $D \subseteq B_{1} \times B_{2}$ and as-maximal element $(a, b) \in D$ we have $a \neq b$ as required.

Finally, suppose $\beta \not \leq \gamma_{2}$, then $\beta \vee \gamma_{2}=\underline{1}_{R}$. Take a $\beta$-block $B, B \cap C^{2} \neq \varnothing$, we have $B \subseteq B_{1} \times \mathbb{A}$ for some $\alpha$-block $B_{1}$. Moreover, by the Claim for any $\alpha$-block $B_{2}$ with $B_{2} \cap C \neq \varnothing$ there is $(a, b) \in B \cap C^{2}$ such that $b \in B_{2}$. By Lemma 22 there is an as-component $E$ of $B$ such that $(a, b)$ above can be chosen from $E$. Choosing $B_{2} \neq B_{1}$ we obtain a required pair.

\section{Separating congruences}

In this section we introduce and study the relationship between prime intervals in the congruence lattice of an algebra, or in congruence lattices of factors in a subdirect products. It was first introduced in [13] and used in the CSP research in [22].

\subsection{Special polynomials, mapping pairs}

We start with several technical results. They demonstrate the connection between minimal sets of an algebra $\mathbb{A}$ and the structure of its graph $\mathcal{G}^{\prime}(\mathbb{A})$. Let $\mathbb{A}$ be an algebra and let $Q_{a b}^{\mathbb{A}}, a, b \in \mathbb{A}$, denote the subdirect product of $\mathbb{A}^{2}$ generated by $\{(x, x) \mid x \in \mathbb{A}\} \cup\{(a, b)\}$.

Lemma 37 (1) $Q_{a b}^{\mathbb{A}}=\left\{(f(a), f(b)) \mid f \in \operatorname{Pol}_{1}(\mathbb{A})\right\}$.

(2) For any $f \in \operatorname{Pol}_{1}(\mathbb{A}),(f(a), f(b)) \in$ tol $_{1}\left(Q_{a b}^{\mathbb{A}}\right)$. In particular, $\left.\right|_{1}\left(Q_{a b}^{\mathbb{A}}\right)=$ 
$\mathrm{Cg}(a, b)$; denote this congruence by $\alpha$.

(3) $Q_{a b}^{\mathbb{A}} \subseteq \operatorname{Cg}(a, b)$.

(4) Let $B_{1}, B_{2}$ be $\alpha$-blocks, and $C_{1}, C_{2}$ as-components of $B_{1}, B_{2}$, respectively, such that $f(a) \in C_{1}$ and $f(b) \in C_{2}$ for a polynomial $f$. Then $C_{1} \times C_{2} \subseteq Q_{a b}^{\mathbb{A}}$.

Proof: (1) follows directly from the definitions.

(2) Take $f \in \operatorname{Pol}_{1}(\mathbb{A})$ and let $f(x)=g\left(x, a_{1}, \ldots, a_{k}\right)$ for a term operation $g$ of $\mathbb{A}$. Then $\left(\begin{array}{l}f(a) \\ f(b)\end{array}\right)=g\left(\left(\begin{array}{l}a \\ b\end{array}\right),\left(\begin{array}{l}a_{1} \\ a_{1}\end{array}\right), \ldots,\left(\begin{array}{l}a_{k} \\ a_{k}\end{array}\right)\right) \in R$ and $\left(\begin{array}{l}f(b) \\ f(b)\end{array}\right)=$ $g\left(\left(\begin{array}{l}b \\ b\end{array}\right),\left(\begin{array}{l}a_{1} \\ a_{1}\end{array}\right), \ldots,\left(\begin{array}{l}a_{k} \\ a_{k}\end{array}\right)\right) \in R$. Thus $(f(a), f(b)) \in \operatorname{tol}_{1}\left(Q_{a b}^{\mathbb{A}}\right)$.

(3) follows from (1), and (4) follows from (2),(3), and the Rectangularity Corollary 34 .

We say that $a$ is $\alpha$-maximal for a congruence $\alpha \in \operatorname{Con}(\mathbb{A})$ if $a$ is as-maximal in the subalgebra $a^{\alpha}$.

Corollary 38 Let $\alpha \in \operatorname{Con}(\mathbb{A})$ and $\underline{0} \prec \alpha$. Then for any $a, b \in \mathbb{A}$ with $a \stackrel{\alpha}{\equiv} b$ and any $\alpha$-maximal $c, d \in \mathbb{A}, c \neq d$, with $c \stackrel{\alpha}{\equiv} d$, belonging to the same as-component of $c^{\alpha}$, there is $f \in \mathrm{Pol}_{1}(\mathbb{A})$ such that $c=f(a), d=f(b)$.

Proof: The result follows from Lemma $37(4)$.

Recall that for $\alpha, \beta \in \operatorname{Con}(\mathbb{A})$ with $\alpha \prec \beta$ a pair $\{a, b\}$ is called an $(\alpha, \beta)$ subtrace if $(a, b) \in \beta-\alpha$ and $a, b \in U$ for some $(\alpha, \beta)$-minimal set $U$.

Corollary 39 Let $\alpha \in \operatorname{Con}(\mathbb{A})$ and $\underline{0} \prec \alpha$, and let $c, d \in \mathbb{A}, c \stackrel{\alpha}{\equiv} d$, be $\alpha$-maximal.

(1) If $c, d$ belong to the same as-component of $c^{\alpha}$, then $\{c, d\}$ is a $(\underline{0}, \alpha)$-subtrace.

(2) If there is a $(\underline{0}, \alpha)$-subtrace $\left\{c^{\prime}, d^{\prime}\right\}$ such that $c^{\prime} \in \operatorname{as}(c)$ and $d^{\prime} \in \operatorname{as}(d)$ then $\{c, d\}$ is a $(\underline{0}, \alpha)$-subtrace as well.

Proof: (1) Take any $(\underline{0}, \alpha)$-minimal set $U$, and $a, b \in U$ with $a \stackrel{\alpha}{\equiv} b$. By Corollary 38 there is $f \in \mathrm{Pol}_{1}(\mathbb{A})$ with $c=f(a), d=f(b)$. By Lemma 14(3) $U^{\prime}=f(U)$ is a $(\underline{0}, \alpha)$-minimal set.

(2) As in (1) one can argue that $\left(c^{\prime}, d^{\prime}\right) \in Q_{a b}^{\mathbb{A}}$, that is, $Q_{a b}^{\mathbb{A}} \cap(\operatorname{as}(c) \times \operatorname{as}(d)) \neq$ $\varnothing$. We then complete by Lemma $37(4)$.

Lemma 40 For any $\alpha \in \operatorname{Con}(\mathbb{A})$ with $\underline{0} \prec \alpha$ such that $|D|>1$ for some ascomponent $D$ of an $\alpha$-block, the prime interval $\underline{0} \prec \alpha$ has type 2 or $\mathbf{3}$.

Proof: Let $a, b \in D$ for an as-component $D$ of an $\alpha$-block. Then by Corollary 38 there is a polynomial $f$ such that $f(a)=b$ and $f(b)=a$. Also, $a, b$ belong to some $(\underline{0}, \alpha)$-minimal set. This rules out types $\mathbf{4}$ and $\mathbf{5}$. Since $\mathbb{A}$ omits type $\mathbf{1}$, this only leaves types $\mathbf{2}$ and $\mathbf{3}$. 
Lemma 41 Let $\alpha \in \operatorname{Con}(\mathbb{A})$ with $\underline{0} \prec \alpha$ be such that some $\alpha$-block contains a semilattice or majority edge. Then the prime interval $(\underline{0}, \alpha)$ has type $\mathbf{3}, \mathbf{4}$ or $\mathbf{5}$.

Proof: We need to show that $(\underline{0}, \alpha)$ does not have type 2 . Let $B$ the $\alpha$-block containing a semilattice or majority edge. Then $B$ contains a non-Abelian subalgebra, which implies $(\underline{0}, \alpha)$ is also non-Abelian.

\subsection{Separation}

Let $\mathbb{A}$ be an algebra, and let $\alpha \prec \beta$ and $\gamma \prec \delta$ be prime intervals in $\operatorname{Con}(\mathbb{A})$. We say that $\alpha \prec \beta$ can be separated from $\gamma \prec \delta$ if there is a unary polynomial $f \in \operatorname{Pol}_{1}(\mathbb{A})$ such that $f(\beta) \nsubseteq \alpha$, but $f(\delta) \subseteq \gamma$. The polynomial $f$ in this case is said to separate $\alpha \prec \beta$ from $\gamma \prec \delta$.

Since we often consider relations rather than single algebras, we also introduce separability in a slightly different way. Let $R$ be a subdirect product of $\mathbb{A}_{1} \times \cdots \times$ $\mathbb{A}_{n}$. Let $i, j \in[n]$ and let $\alpha_{i} \prec \beta_{i}, \alpha_{j} \prec \beta_{j}$ be prime intervals in $\operatorname{Con}\left(\mathbb{A}_{i}\right)$ and $\operatorname{Con}\left(\mathbb{A}_{j}\right)$, respectively. Interval $\alpha_{i} \prec \beta_{i}$ can be separated from $\alpha_{j} \prec \beta_{j}$ if there is a unary polynomial $f$ of $R$ such that $f\left(\beta_{i}\right) \nsubseteq \alpha_{i}$ but $f\left(\beta_{j}\right) \subseteq \alpha_{j}$. Similarly, the polynomial $f$ in this case is said to separate $\alpha_{i} \prec \beta_{i}$ from $\alpha_{j} \prec \beta_{j}$

First, we observe a connection between separation in a single algebra and in relations.

Lemma 42 Let $R$ be the binary equality relation on $\mathbb{A}$. Let $\alpha_{1}=\alpha, \beta_{1}=\beta$ be viewed as congruences of the first factor of $R$, and $\alpha_{2}=\gamma, \beta_{2}=\delta$ as congruences of the second factor of $R$. Prime interval $\alpha \prec \beta$ can be separated from $\gamma \prec \delta$ as intervals in $\operatorname{Con}(\mathbb{A})$ if and only if $\alpha_{1} \prec \beta_{1}$ can be separated from $\alpha_{2} \prec \beta_{2}$ in $R$.

Proof: Note that for any polynomial $f$ its action on the first and second projections of $R$ is the same polynomial of $\mathbb{A}$. Therefore $\alpha \prec \beta$ can be separated from $\gamma \prec \delta$ in $\operatorname{Con}(\mathbb{A})$ if and only if, there is $f \in \operatorname{Pol}_{1}(\mathbb{A}), f(\beta) \nsubseteq \alpha$ while $f(\delta) \subseteq \gamma$. This condition can be expressed as follows: there is $f \in \operatorname{Pol}_{1}(R), f\left(\beta_{1}\right) \nsubseteq \alpha_{1}$ while $f\left(\beta_{2}\right) \subseteq \alpha_{2}$, which precisely means that $\alpha_{1} \prec \beta_{1}$ can be separated from $\alpha_{2} \prec \beta_{2}$ in $R$.

In what follows when proving results about separation we will always assume that we deal with a relation - a subdirect product — and that the prime intervals in question are from congruence lattices of different factors of the subdirect product. If this is not the case, one can duplicate the factor containing the prime intervals and apply Lemma 42

Let $R$ be a subdirect product of $\mathbb{A}_{1} \times \cdots \times \mathbb{A}_{n}, I \subseteq[n]$, and let $f$ be a polynomial of $\operatorname{pr}_{I} R$, that is, there are a term operation $g$ of $R$ and $\mathbf{a}_{1}, \ldots, \mathbf{a}_{k} \in \operatorname{pr}_{I} R$ 
such that $f\left(x_{1}, \ldots, x_{\ell}\right)=g\left(x_{1}, \ldots, x_{\ell}, \mathbf{a}_{1}, \ldots, \mathbf{a}_{k}\right)$. The tuples $\mathbf{a}_{i}$ can be extended to tuples $\mathbf{a}_{i}^{\prime} \in R$. Then the polynomial of $R$ given by $f\left(x_{1}, \ldots, x_{\ell}\right)=$ $g\left(x_{1}, \ldots, x_{\ell}, \mathbf{a}_{1}^{\prime}, \ldots, \mathbf{a}_{k}^{\prime}\right)$ is said to be an extension of $f$ to a polynomial of $R$.

Lemma 43 Let $R$ be a subdirect product of $\mathbb{A}_{1} \times \cdots \times \mathbb{A}_{n}, i, j \in[n]$, and $\alpha_{i} \prec \beta_{i}$, $\alpha_{j} \prec \beta_{j}$ for $\alpha_{i}, \beta_{i} \in \operatorname{Con}\left(\mathbb{A}_{i}\right), \alpha_{j}, \beta_{j} \in \operatorname{Con}\left(\mathbb{A}_{j}\right)$. Let also a unary polynomial $f$ of $R$ separate $\alpha_{i} \prec \beta_{i}$ from $\alpha_{j} \prec \beta_{j}$. Then $f$ can be chosen idempotent and such that $f\left(\mathbb{A}_{i}\right)$ is a $\left(\alpha_{i}, \beta_{i}\right)$-minimal set.

Proof: Let $g$ be a polynomial separating $\left(\alpha_{i}, \beta_{i}\right)$ from $\left(\alpha_{j}, \beta_{j}\right)$. Since $g\left(\beta_{i}\right) \nsubseteq$ $\alpha_{i}$, by Lemma 14(6) there is an $\left(\alpha_{i}, \beta_{i}\right)$-minimal set $U$ such that $g\left(\left.\beta_{i}\right|_{U}\right) \nsubseteq \alpha_{i}$. Let $V=g(U)$, by Lemma 14(2) $V$ is a $\left(\alpha_{i}, \beta_{i}\right)$-minimal set. Let $h$ be a unary polynomial such that $h$ maps $V$ onto $U$ and $\left.h \circ g\right|_{U}$ is the identity mapping. Let also $h^{\prime}$ be an extension of $h$ to a polynomial of $R$. Then $h^{\prime} \circ g$ separates $i$ from $j$. Now $f$ can be chosen to be an appropriate power of $h^{\prime} \circ g$.

For a subdirect product $R \subseteq \mathbb{A}_{1} \times \cdots \times \mathbb{A}_{n}$ the relation 'cannot be separated' on prime intervals of the $\mathbb{A}_{i} \mathrm{~s}$ is clearly reflexive and transitive. If the algebras $\mathbb{A}_{i}$ are Mal'tsev, it is also symmetric (for partial results see [13, 22]). Moreover, it can be shown that it remains 'almost' symmetric when the $\mathbb{A}_{i}$ s contain no majority edges. In the general case however the situation is more complicated. Next we introduce conditions that make the 'cannot be separated' relation to some extent symmetric, at least in what concerns our algorithm, as it will be demonstrated in Lemma 46

Let $\beta_{i} \in \operatorname{Con}\left(\mathbb{A}_{i}\right)$, let $B_{i}$ be a $\beta_{i}$-block for $i \in[n]$, and let $B_{i}^{\prime}=\operatorname{pr}_{i}(R \cap$ $\bar{B})$. Let also $\mathcal{U}$ be a set of unary polynomials of $R, i$ an element from $[n]$, and $\alpha, \beta \in \operatorname{Con}\left(\mathbb{A}_{i}\right)$ with $\alpha \prec \beta \leq \beta_{i}$. Let $T_{\mathbb{A}_{i}}\left(a^{\prime}, b^{\prime} ; \alpha, \beta, \mathcal{U}\right) \subseteq \beta / \alpha \subseteq\left(\mathbb{A}_{i} / \alpha\right)^{2}$ for $a^{\prime}, b^{\prime} \in B_{i}^{\prime} / \alpha,\left(a^{\prime}, b^{\prime}\right) \in \beta-\alpha$, denote the set of pairs $(a, b) \in \beta / \alpha$ such that there is a polynomial $g \in \mathcal{U}$ satisfying the following conditions: $g\left(\left\{a^{\prime}, b^{\prime}\right\}\right)=\{a, b\}$ and $g\left(\mathbb{A}_{i}\right)$ is a $(\alpha, \beta)$-minimal set. Note that these conditions imply that $\{a, b\}$ is a $(\alpha, \beta)$-subtrace or $a=b$. We say that $\alpha$ and $\beta$ are $\mathcal{U}$-chained in $R$ with respect to $\bar{\beta}, \bar{B}$ if for any $a^{\prime}, b^{\prime} \in B_{i}^{\prime} / \alpha$ with $\left(a^{\prime}, b^{\prime}\right) \in \beta-\alpha$, the following conditions hold:

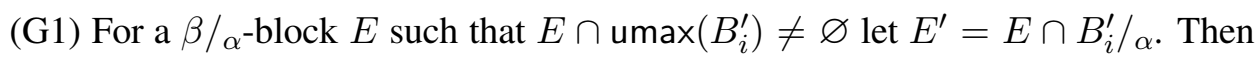
$(a, b) \in T_{\mathbb{A}_{i}}\left(a^{\prime}, b^{\prime} ; \alpha, \beta, \mathcal{U}\right)$ for any $a, b$ from the same as-component of $E^{\prime}$.

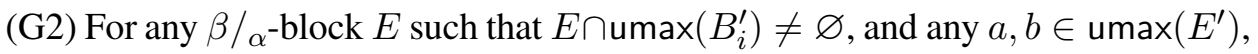
where $E^{\prime}=E \cap B_{i}^{\prime} / \alpha$, there is a sequence $a=a_{1}, \ldots, a_{k}=b$ in $E^{\prime}$ such that $\left\{a_{i}, a_{i+1}\right\} \in T_{\mathbb{A}_{i}}\left(a^{\prime}, b^{\prime} ; \alpha, \beta, \mathcal{U}\right)$ for any $i \in[k-1]$.

Also, if for elements $a, b$ and any $a^{\prime}, b^{\prime}$ there is a sequence of elements satisfying (G2), we say that $a$ and $b$ are subtrace connected; congruences $\alpha, \beta, \bar{\beta}$, congruence classes $\bar{B}$, and set of polynomials $\mathcal{U}$ will always be clear from the context in this case. Observe that $\mathcal{U}$-chaining amounts to saying that polynomials from $\mathcal{U}$ do not 
allow any congruences of $\beta$-blocks viewed as subalgebras between $\alpha$ and $\beta$, at least where u-maximal elements are concerned.

A unary polynomial $f$ is said to be $\bar{B}$-preserving if $f(\bar{B}) \subseteq \bar{B}$. We call relation $R$ chained with respect to $\bar{\beta}, \bar{B}$ if

(Q1) for any $\alpha, \beta \in \operatorname{Con}\left(\mathbb{A}_{i}\right), i \in[n]$, such that $\alpha \prec \beta \leq \beta_{i}$, congruences $\alpha$ and $\beta$ are $\mathcal{U}_{B}$-chained in $R$, where $\mathcal{U}_{B}$ is the set of all $\bar{B}$-preserving polynomials of $R$

(Q2) for any $\alpha, \beta \in \operatorname{Con}\left(\mathbb{A}_{i}\right), \gamma, \delta \in \operatorname{Con}\left(\mathbb{A}_{j}\right), i, j \in[n]$, such that $\alpha \prec \beta \leq \beta_{i}$, $\gamma \prec \delta \leq \beta_{j}$, and $(\alpha, \beta)$ can be separated from $(\gamma, \delta)$, congruences $\alpha$ and $\beta$ are $\mathcal{U}^{*}$-chained in $R$, where $\mathcal{U}^{*}$ is the set of all $\bar{B}$-preserving polynomials of $R$ such that $g(\delta) \subseteq \gamma$.

Polynomials from $\mathcal{U}^{*}$ in condition $(\mathrm{Q} 2)$ will be called $(\gamma, \delta, \bar{B})$-good.

Lemma 44 (1) Any constant polynomial from $\bar{B} \cap R$ is $(\gamma, \delta, \bar{B})$-good.

(2) If $f$ is a $k$-ary term function of $R$ and $g_{1}, \ldots, g_{k}$ are $(\gamma, \delta, \bar{B})$-good polynomials, then $f\left(g_{1}(x), \ldots, g_{k}(x)\right)$ is $(\gamma, \delta, \bar{B})$-good.

(3) Let $T\left(a^{\prime}, b^{\prime}\right)$ denote $T_{\mathbb{A}_{i}}\left(a^{\prime}, b^{\prime} ; \alpha, \beta, \mathcal{U}\right)$ for $\mathcal{U} \in\left\{\mathcal{U}_{\bar{B}}, \mathcal{U}^{*}\right\}$. If $\{a, b\} \in T\left(a^{\prime}, b^{\prime}\right)$ then $T(a, b) \subseteq T\left(a^{\prime}, b^{\prime}\right)$.

(4) If there is a $\beta / \alpha^{-}$-block $E$ such that $E \cap \operatorname{umax}\left(B_{i}^{\prime}\right) \neq \varnothing$, let $E^{\prime}=E \cap B_{i}^{\prime} / \alpha$, and if $E^{\prime}$ contains a nontrivial as-component, then there is a set $T \subseteq \beta / \alpha$ such that $T \subseteq T\left(a^{\prime}, b^{\prime}\right)$ for any $a^{\prime}, b^{\prime} \in B_{i}^{\prime} /{ }_{\alpha}, a^{\prime} \stackrel{\beta / \alpha}{=} b^{\prime}$ and $T$ satisfies conditions $(G 1),(G 2)$ for $T\left(a^{\prime}, b^{\prime}\right)$.

(5) Let $a^{\prime}, b^{\prime} \in B_{i}^{\prime} / \alpha, a^{\prime} \stackrel{\beta / \alpha}{=} b^{\prime}$ be such that $T\left(a^{\prime}, b^{\prime}\right)$ is minimal among sets of this form. Then for any $(a, b) \in T\left(a^{\prime}, b^{\prime}\right)$ there is $h \in \mathcal{U}$ such that $h$ is idempotent and $h(a) \stackrel{\alpha}{\equiv} a, h(b) \stackrel{\alpha}{=} b$.

Proof: Items (1),(2) are straightforward.

(3) Let $\left\{a^{\prime \prime}, b^{\prime \prime}\right\} \in T(a, b)$. Then there are polynomials $f, g \in \mathcal{U}$ with $\{a, b\}=$ $f\left(\left\{a^{\prime}, b^{\prime}\right\}\right)$ and $\left\{a^{\prime \prime}, b^{\prime \prime}\right\}=g(\{a, b\})$. Then $g \circ f \in \mathcal{U}$ by item (2) or definition and $g \circ f\left(\left\{a^{\prime}, b^{\prime}\right\}\right)=\left\{a^{\prime \prime}, b^{\prime \prime}\right\}$.

(4) Take $a, b \in C$ where $C$ is a nontrivial as-component in $E^{\prime}$. By (G2) $\{a, b\} \in T\left(a^{\prime}, b^{\prime}\right)$ for any appropriate $a^{\prime}, b^{\prime}$. Therefore by (3) $T=T(a, b) \subseteq$ $T\left(a^{\prime}, b^{\prime}\right)$.

(5) Let $\{a, b\} \in T\left(a^{\prime}, b^{\prime}\right)$. Then by (3) $T(a, b) \subseteq T\left(a^{\prime}, b^{\prime}\right)$, and therefore by the minimality of $T\left(a^{\prime}, b^{\prime}\right)$ we get $T(a, b)=T\left(a^{\prime}, b^{\prime}\right)$. The result follows by definition of $T\left(a^{\prime}, b^{\prime}\right)$.

The next lemma shows how we will use the property of being chained. 
Lemma 45 Let $R$ be a subdirect product of $\mathbb{A}_{1}, \ldots, \mathbb{A}_{n}$ chained with respect to $\bar{\beta}, \bar{B}$, where $\beta_{i} \in \operatorname{Con}\left(\mathbb{A}_{i}\right)$ and $B_{i}$ is a $\beta_{i}$-block, and $R^{\prime}=R \cap\left(B_{1} \times \cdots \times B_{n}\right)$, $B_{i}^{\prime}=\operatorname{pr}_{i} R^{\prime}$. Let also lk be the link congruence of $B_{i}^{\prime}$ with respect to $\operatorname{pr}_{i j} R^{\prime}$ for some $i, j \in[n]$, and $\delta=\mathrm{Cg}(\mathrm{lk})$ the congruence of $\mathbb{A}_{i}$ generated by $\mathrm{lk}$. Then for any $\gamma \in \operatorname{Con}\left(\mathbb{A}_{i}\right)$ with $\gamma \prec \delta$ it holds $\left.(\delta / \gamma)\right|_{\mathrm{umax}(E)}=\left.(\mathrm{Ik} / \gamma)\right|_{\mathrm{umax}(E)}$ for every $\left.\delta\right|_{B_{i}^{\prime}}$-block E u-maximal in $B_{i}^{\prime} / \delta$.

Proof: If $\gamma \prec \delta$ then by the choice of $\delta$ there are $a, b \in B_{i}^{\prime}$ with $(a, b) \in$ $\delta-\gamma$. Let $E$ be the intersection of $B_{i}^{\prime}$ with a $\delta$-block. We apply condition (Q1) to $\alpha=\gamma \wedge \beta_{i}$ and $\beta=\delta \wedge \beta_{i}$. By condition (Q1) for any $a^{\prime}, b^{\prime} \in \operatorname{umax}(E)$ there is a sequence $a^{\prime}=a_{1}, \ldots, a_{k}=b^{\prime}$ such that $\left\{a_{\ell}, a_{\ell+1}\right\}=f_{\ell}(\{a, b\})$ for some $\bar{B}$-preserving polynomial $f_{\ell}$ for each $\ell \in[k-1]$. This means that $\left(a_{\ell}, a_{\ell+1}\right) \in \mathrm{lk}$, and so $\left(a^{\prime}, b^{\prime}\right) \in \mathrm{lk}$.

The following lemma establishes the weak symmetricity of separability relation mentioned before.

Lemma 46 Let $R$ be a subdirect product of $\mathbb{A}_{1} \times \cdots \times \mathbb{A}_{n}, \beta_{i} \in \operatorname{Con}\left(\mathbb{A}_{i}\right), B_{i} a$ $\beta_{i}$-block such that $R$ is chained with respect to $\bar{\beta}, \bar{B} ; R^{\prime}=R \cap \bar{B}, B_{i}^{\prime}=\operatorname{pr}_{i} R^{\prime}$. Let also $\alpha \prec \beta \leq \beta_{1}, \gamma \prec \delta=\beta_{2}$, where $\alpha, \beta \in \operatorname{Con}\left(\mathbb{A}_{1}\right), \gamma, \delta \in \operatorname{Con}\left(\mathbb{A}_{2}\right)$. If $B_{2}^{\prime} / \gamma$ has a nontrivial as-component $C^{\prime}$ and $(\alpha, \beta)$ can be separated from $(\gamma, \delta)$, then there is a $\bar{B}$-preserving polynomial $g$ such that $g\left(\left.\beta\right|_{B_{1}^{\prime}}\right) \subseteq \alpha$ and $g(\delta) \nsubseteq$ $\gamma$. Moreover, for any $c, d \in C^{\prime}$ polynomial $f$ can be chosen such that $f(c)=$ $c, f(d)=d$.

Proof: As is easily seen, we can assume that $\alpha, \gamma$ are equality relations. We need to show that there is $g$ such that $g$ collapses $\beta$ but does not collapse $\beta_{2}=\delta$.

First we show that there are $c, d \in B_{2}^{\prime}$ such that for any $\left.(a, b) \in \beta\right|_{B_{1}^{\prime}}$ there is a polynomial $h^{a b}$ of $R$ such that

(1) $h^{a b}$ is idempotent;

(2) $h^{a b}(a)=h^{a b}(b)$;

(3) $h^{a b}(c)=c, h^{a b}(d)=d$.

We consider two cases.

CASE 1. There is an element $c$ from a nontrivial as-component of $B_{2}^{\prime}$ such that $(a, c, \mathbf{b}) \in R^{\prime}$ for some $a \in B^{\prime}$, a $\beta$-block such that $B^{\prime} \cap \operatorname{umax}\left(B_{1}^{\prime}\right) \neq \varnothing$ and $\left|\operatorname{umax}\left(B^{\prime} \cap B_{1}^{\prime}\right)\right|>1$.

First, we choose $d$ to be any element other than $c$ of the nontrivial as-component $C^{\prime}$ of $B_{2}^{\prime}$ containing $c$. Let $T_{1}$ be a minimal set of $(\alpha, \beta)$-subtraces as in Lemma 44 (4) 
for $\mathcal{U}^{*}$, the set of $(\gamma, \delta, \bar{B})$-good polynomials. We start with the case when $(a, b) \in$ $T_{1}$. Even more specifically, as $c$ is as-maximal in $B_{2}^{\prime}$, by the Maximality Lemma27(5) $a$ can be chosen from $\operatorname{umax}\left(B^{\prime} \cap B_{1}^{\prime}\right)$. Take an $(\alpha, \beta)$-subtrace $\{a, b\} \in T_{1}$, such a subtrace exists by condition $(\mathrm{Q} 1)$.

By $Q^{*} \subseteq \mathbb{A}_{1}^{2} \times \mathbb{A}_{2}^{2} \times R$ we denote the relation generated by $\{(a, b, c, d, \mathbf{a})\} \cup$ $\{(x, x, y, y, \mathbf{z}) \mid \mathbf{z} \in R, \mathbf{z}[1]=x, \mathbf{z}[2]=y\}$, where $\mathbf{a}$ is an arbitrary element from $R^{\prime}$. Let $Q=\operatorname{pr}_{1234} Q^{*}$ and $Q^{\prime}=\operatorname{pr}_{1234}\left(Q^{*} \cap\left(B_{1}^{\prime} \times B_{1}^{\prime} \times B_{2}^{\prime} \times B_{2}^{\prime} \times \bar{B}\right)\right)$. Observe that $Q$ is exactly the set of quadruples $(f(a), f(b), f(c), f(d))$ for unary polynomials $f$ of $R$ and $Q^{\prime}$ is exactly the set of quadruples $(f(a), f(b), f(c), f(d))$ for $\bar{B}$-preserving unary polynomials $f$ of $R$. We prove that $Q^{\prime}$ contains a quadruple of the form $\left(a^{\prime}, a^{\prime}, c, d\right)$; the result then follows.

Let also $Q_{1}=\operatorname{pr}_{1,2} Q=Q_{a b}^{\mathbb{A}_{1}}, Q_{2}=\operatorname{pr}_{3,4} Q=Q_{c d}^{\mathbb{A}_{2}}$; set $Q_{1}^{\prime}=\operatorname{pr}_{1,2} Q^{\prime}$, $Q_{2}^{\prime}=\operatorname{pr}_{3,4} Q^{\prime}$. Note that $\operatorname{pr}_{1} Q^{\prime}=\operatorname{pr}_{2} Q^{\prime}=B_{1}^{\prime}$ and $\operatorname{pr}_{3} Q^{\prime}=\operatorname{pr}_{4} Q^{\prime}=B_{2}^{\prime}$, because $\operatorname{pr}_{12} R^{\prime} \subseteq \operatorname{pr}_{13} Q^{\prime}, \operatorname{pr}_{24} Q^{\prime}$. Let $\mathrm{lk}_{1}, \mathrm{Ik}_{2}$ denote the link congruences of $Q^{\prime}$ viewed as a subdirect product of $Q_{1}^{\prime}$ and $Q_{2}^{\prime}$. Note that these congruences may be different from the link congruences of $Q$ restricted to $Q_{1} \cap\left(B_{1}^{\prime} \times B_{1}^{\prime}\right), Q_{2} \cap\left(B_{2}^{\prime} \times B_{2}^{\prime}\right)$, respectively. We show that $\left(a^{\prime}, a^{\prime}\right)$ for some $a^{\prime} \in B_{1}^{\prime}$ is as-maximal in a lk $\mathrm{k}_{1}$-block, $(c, d)$ is as-maximal in a $\mathrm{Ik}_{2}$-block, and $Q^{\prime} \cap\left(\operatorname{as}\left(a^{\prime}, a^{\prime}\right) \times \operatorname{as}(c, d)\right) \neq \varnothing$. By the Rectangularity Corollary 34 this implies the result.

CLAIM 1. $\left.(\alpha \times \beta)\right|_{Q_{1}^{\prime}} \subseteq \mathrm{lk}_{1}$ and $\left.(\gamma \times \delta)\right|_{Q_{2}^{\prime}} \subseteq \mathrm{lk}_{2}$.

Relation $Q^{\prime}$ contains tuples $(a, b, c, d),\left(a, b, c^{\prime}, c^{\prime}\right),\left(a, a, c^{\prime}, c^{\prime}\right),(a, a, c, c)$ for some $c^{\prime} \in B_{2}^{\prime}$. Indeed, $(a, b, c, d) \in Q^{\prime}$ by definition, $(a, a, c, c) \in Q$ because $(a, c, \mathbf{b}) \in R$, and $\left(a, b, c^{\prime}, c^{\prime}\right),\left(a, a, c^{\prime}, c^{\prime}\right)$ can be chosen to be the images of $(a, b, c, d)$ and $(a, a, c, c)$, respectively, under a $\bar{B}$-preserving polynomial $g^{a b}$ such that $g^{a b}(a)=a, g^{a b}(b)=b$ and $g^{a b}(\delta) \subseteq \gamma$. Such a polynomial exists because $R$ is chained and because $(\alpha, \beta)$ can be separated from $(\gamma, \delta)$. This implies that $(c, d) \stackrel{\mathrm{Ik}_{2}}{\equiv}(c, c)$. Let $\eta_{1}, \eta_{2}$ be congruences of $Q_{1}, Q_{2}$ generated by $((a, b),(a, a))$ and $((c, d),(c, c))$, respectively. Then

$$
\left.\eta_{1}\right|_{Q_{1}^{\prime}}=\left.(\alpha \times \beta)\right|_{Q_{1}^{\prime}}, \quad \text { and }\left.\quad \eta_{2}\right|_{Q_{2}^{\prime}}=\left.(\gamma \times \delta)\right|_{Q_{2}^{\prime}} .
$$

Indeed, in the case of, say, $\alpha \times \beta$, relation $Q_{1}^{\prime}$ consists of pairs $(g(a), g(b))$ for a $\bar{B}$ preserving unary polynomial $g$ of $\mathbb{A}_{1}$. Since $(a, b) \stackrel{\alpha \times \beta}{\equiv}(a, a)$, for any $\left(a^{\prime}, b^{\prime}\right) \in Q_{1}^{\prime}$ it holds that

$$
\left(a^{\prime}, b^{\prime}\right)=(g(a), g(b)) \stackrel{\eta_{1}}{=}(g(a), g(a))=\left(a^{\prime}, a^{\prime}\right) .
$$

Since $(a, b),(a, a)$ are in the same $\mathrm{lk}_{1}$-block, $\left.(\alpha \times \beta)\right|_{Q_{1}^{\prime}} \subseteq \mathrm{lk}_{1}$.

For $Q_{2}^{\prime}$ and $\gamma \times \delta$ the argument is similar. Observing from the same tuples as before that $(c, d) \stackrel{\mathrm{lk}_{2}}{=}(c, c)$, we prove $\left.(\gamma \times \delta)\right|_{Q_{2}^{\prime}} \subseteq \mathrm{I}_{2}$ by a similar argument. Claim 1 is proved. 
Claim 2. Let $E=B^{\prime} \cap B_{1}^{\prime}$, where $B^{\prime}$ is the $\beta$-block containing $a, b$. Then $\left.(\beta \times \beta)\right|_{\operatorname{umax}(E) \times \operatorname{umax}(E)} \subseteq \mathrm{lk}_{1}$.

By the assumption for any $(\alpha, \beta)$-subtrace $\left(a^{\prime}, b^{\prime}\right) \in T_{1} \cap E^{2}$ there is a $\bar{B}$ preserving polynomial $g^{a^{\prime} b^{\prime}}$ satisfying $g^{a^{\prime} b^{\prime}}\left(a^{\prime}\right)=a^{\prime}, g^{a^{\prime} b^{\prime}}\left(b^{\prime}\right)=b^{\prime}$, and $g^{a^{\prime} b^{\prime}}\left(B_{2}^{\prime}\right)=$ $\left\{c^{\prime}\right\} \subseteq B_{2}^{\prime}$. Applying $g^{a^{\prime} b^{\prime}}$ to tuples $(a, b, c, d),(a, a, c, c)$, and $\left(b, b, d^{\prime}, d^{\prime}\right)$ for any $d^{\prime}$ such that $(b, d) \in \operatorname{pr}_{12} R^{\prime}$, we obtain $\left(a^{\prime}, b^{\prime}, c^{\prime}, c^{\prime}\right),\left(a^{\prime}, a^{\prime}, c^{\prime}, c^{\prime}\right),\left(b^{\prime}, b^{\prime}, c^{\prime}, c^{\prime}\right) \in$

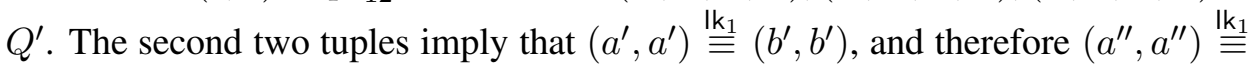
$\left(b^{\prime \prime}, b^{\prime \prime}\right)$ for any $a^{\prime \prime}, b^{\prime \prime} \in \operatorname{umax}(E)$. Along with Claim 1 this proves the result.

CLAIM 3. $(c, d)$ is as-maximal in a $\mathrm{lk}_{2}$-block.

If for some $e, e^{\prime} \in B_{2}^{\prime}$ we have $(e, e) \stackrel{\text { lk }}{=}\left(e^{\prime}, e^{\prime}\right)$, then, as $\left(e, e^{\prime}\right)$ generates $\delta$, for any $(\gamma, \delta)$-subtrace $\left\{e^{\prime \prime}, e^{\prime \prime \prime}\right\} \in T_{\mathbb{A}_{2}}\left(e, e^{\prime}\right)=T_{\mathbb{A}_{2}}\left(e, e^{\prime} ; \gamma, \delta, \mathcal{U}_{\bar{B}}\right)$ there is a $\bar{B}$ preserving polynomial $f^{\prime}$ with $f^{\prime}\left(\left\{e, e^{\prime}\right\}\right)=\left\{e^{\prime \prime}, e^{\prime \prime \prime}\right\}$. Applying this polynomial to the tuples witnessing that $(e, e) \stackrel{\mathrm{lk}_{2}}{=}\left(e^{\prime}, e^{\prime}\right)$ we get $\left(e^{\prime \prime}, e^{\prime \prime}\right) \stackrel{\text { l } \mathrm{k}_{2}}{=}\left(e^{\prime \prime \prime}, e^{\prime \prime \prime}\right)$. Therefore by condition (Q1) all tuples of the form $(x, x), x \in \operatorname{umax}\left(B_{2}^{\prime}\right)$, are $\mathrm{lk}_{2}$-related. Since by condition (G1) $\{c, d\}$ is a $(\gamma, \delta)$-subtrace from $T_{\mathbb{A}_{2}}(c, d) \subseteq T_{\mathbb{A}_{2}}\left(e, e^{\prime}\right)$, using Claim 1 this implies that $\left.\mathrm{lk}_{2}\right|_{Q^{\prime \prime}}=\left.(\delta \times \delta)\right|_{Q^{\prime \prime}}$, where $Q^{\prime \prime}=Q_{2}^{\prime} \cap\left(\operatorname{umax}\left(B_{2}^{\prime}\right) \times\right.$ umax $\left(B_{2}^{\prime}\right)$ ). In particular, $C^{\prime} \times C^{\prime}$, where $C^{\prime}$ is the as-component of $B_{2}^{\prime}$ containing $c, d$, is contained in $Q_{2}^{\prime}$, and is contained in a lk ${ }_{2}$-block. All elements of $C^{\prime} \times C^{\prime}$ are as-maximal in $Q^{\prime \prime}$.

Otherwise, since the inclusion $\left.(\gamma \times \delta)\right|_{Q_{2}^{\prime}} \subseteq \mathrm{Ik}_{2}$ implies that if $\left(c_{1}, d_{1}\right) \stackrel{\mathrm{Ik}_{2}}{=}$ $\left(c_{2}, d_{2}\right)$ then $\left(c_{1}, c_{1}\right) \stackrel{\mathrm{Ik}_{2}}{=}\left(c_{2}, c_{2}\right)$, by Claim 1 we have $\left.\mathbf{I k}_{2}\right|_{Q^{\prime \prime}}=\left.(\gamma \times \delta)\right|_{Q^{\prime \prime}}$. In particular, $\{c\} \times C^{\prime}$ is contained in a $\mathrm{lk}_{2}$-block. Since $c, d$ are as-maximal, $(c, d)$ is as-maximal in this $\mathrm{lk}_{2}$-block. Claim 3 is proved.

By the Maximality Lemma 27(5) there is an element $\left(a^{\prime}, b^{\prime}\right)$ as-maximal in a $\mathrm{Ik}_{1}$-block $D$ such that $\left(a^{\prime}, b^{\prime}, c, d\right) \in Q^{\prime}$. If $a^{\prime}=b^{\prime}$ then we are done. Otherwise by condition (G1) and Lemma 44 $(3)\left\{a^{\prime}, b^{\prime}\right\}$ is an $(\alpha, \beta)$-subtrace from $T_{1}$, also $\left(a^{\prime}, c\right) \in R$ because $\operatorname{pr}_{1,3} Q \subseteq R$, and we can replace $a, b$ with $a^{\prime}, b^{\prime}$. Observe that if we show the existence of a polynomial $g$ such that $g\left(a^{\prime}\right)=g\left(b^{\prime}\right)$ and $g(c)=c$, $g(d)=d$, this will witness the existence of $g^{\prime}$ with $g^{\prime}(a)=g^{\prime}(b)$ and $g^{\prime}(c)=c$, $g^{\prime}(d)=d$. Let $E=a^{\prime \beta} \cap B_{1}^{\prime}$. Note that by Claim $\left.2(\beta \times \beta)\right|_{\operatorname{umax}(E) \times \operatorname{umax}(E)} \subseteq \mathrm{Ik}_{1}$ and by the Rectangularity Corollary $34 \operatorname{umax}(E) \times$ as $(c, d) \subseteq Q^{\prime}$. Therefore, again $a^{\prime}, b^{\prime}$ can be chosen as-maximal in $E$. We use $a, b$ for $a^{\prime}, b^{\prime}$ from now on.

Claim 4. $(a, a)$ is as-maximal in $Q_{1}^{\prime \prime}=Q_{1}^{\prime} \cap(E \times E)$.

Let $\eta_{1}, \eta_{2}$ be the link congruences of $B_{1}^{\prime}, B_{2}^{\prime}$, respectively, with respect to $Q_{1}^{\prime}$; as $Q_{1}^{\prime} \subseteq Q_{a b}^{\mathbb{A}_{1}}$ we have $\eta_{1}, \eta_{2} \leq \beta$. On the other hand, since $Q_{1}^{\prime}$ consists of pairs 
of the form $(x, x)$ and $(\alpha, \beta)$-subtraces, and since umax $(E)$ belongs to a block of the transitive closure of $T_{1}$, it is easy to see that $\operatorname{umax}(E)$ is a subset of both a $\eta_{1}$ and $\eta_{2}$-blocks. Indeed, let $e, e^{\prime} \in \operatorname{umax}(E)$ and $e=e_{1}, \ldots, e_{k}=e^{\prime}$ are such that $\left\{e_{i}, e_{i+1}\right\} \in T_{1}$. This means that either $\left(e_{i}, e_{i+1}\right) \in Q_{1}^{\prime}$ or $\left(e_{i+1}, e_{i}\right) \in Q_{1}^{\prime}$. Since $\left(e_{i}, e_{i}\right),\left(e_{i+1}, e_{i+1}\right) \in Q_{1}^{\prime}$ by construction, in either case we have $\left(e_{i}, e_{i+1}\right) \in$ $\eta_{1}, \eta_{2}$.

Let $E^{\prime}$ be the as-component of $E$ containing $a$; such an as-component exists by the choice of $a, b$. As $(a, a) \in Q_{1}^{\prime} \cap\left(E^{\prime} \times E^{\prime}\right) \neq \varnothing$, by the Rectangularity Corollary $34 E^{\prime} \times E^{\prime} \subseteq Q_{1}^{\prime}$. Since $E^{\prime}$ is an as-component in $E$, by Lemma 28 $E^{\prime} \times E^{\prime}$ is an as-component in $Q_{1}^{\prime \prime}$. In particular $(a, a)$ is as-maximal in $Q_{1}^{\prime \prime}$. Claim 4 is proved.

Claim 5. $(a, a, c, d) \in Q^{\prime}$.

To prove this claim we find a subalgebra $Q^{\prime \prime}$ of $Q^{\prime}$ such that it is linked enough and both $(a, a)$ and $(c, d)$ belong to as-components of $\operatorname{pr}_{12} Q^{\prime \prime}, \operatorname{pr}_{34} Q^{\prime \prime}$, respectively, and then apply the Rectangularity Corollary 34.

Let $F$ be the as-component of the $\mathrm{Ik}_{2}$-block containing $(c, d)$. By Claim 3 it is

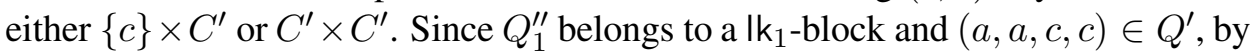
the Maximality Lemma27(4) for any $\left(a^{\prime}, b^{\prime}\right) \in E^{\prime} \times E^{\prime}$ there are $\left(c^{\prime}, d^{\prime}\right) \in F$ such that $\left(a^{\prime}, b^{\prime}, c^{\prime}, d^{\prime}\right) \in Q^{\prime}$. Now consider $Q^{\prime \prime}=Q^{\prime} \cap\left(E \times E \times B_{2}^{\prime} \times B_{2}^{\prime}\right)$. Clearly, $Q_{1}^{\prime} \subseteq$ $\operatorname{pr}_{12} Q^{\prime \prime}$. Also, since $(a, b)$ is as-maximal in a $\mathbf{l k}_{1}$-block, by the Rectangularity Corollary $34\{(a, b)\} \times F \subseteq Q^{\prime \prime}$, implying $F \subseteq \operatorname{pr}_{34} Q^{\prime \prime}$. If $\theta_{1}, \theta_{2}$ denote the link congruences of $\operatorname{pr}_{12} Q^{\prime \prime}, \operatorname{pr}_{34} Q^{\prime \prime}$ with respect to $Q^{\prime \prime}$, the observation above implies that the as-components of $\operatorname{pr}_{12} Q^{\prime \prime}$ containing $(a, a)$ and $(a, b)$ belong to the same $\theta_{1}$-block, and $F$ belongs to a $\theta_{2}$-block. Therefore again by the Rectangularity Corollary 34 we get $\left(E^{\prime} \times E^{\prime}\right) \times\left(\{c\} \times C^{\prime}\right) \subseteq Q^{\prime}$, in particular $(a, a, c, d) \in Q$. Thus, there is a polynomial $h$ such that $h(a)=h(b)=a$ and $h(c)=c, h(d)=d$.

So far we have proved that for any subtrace $(a, b) \in T_{1}$, where $a$ is a fixed element such that $(a, c) \in \operatorname{pr}_{12} R^{\prime}$, there is a polynomial $h^{a b}$ satisfying the conditions stated in the beginning of the proof.

Claim 6. For every $(\alpha, \beta)$-subtrace $\left\{a^{\prime}, b^{\prime}\right\}$ from $T_{1} \cap(E \times E)$ (recall that $\left.E=a^{\beta} \cap B_{1}^{\prime}\right)$ there is a polynomial $h$ such that $h(a)=h(b)$ and $h(c)=c, h(d)=$ $d$.

Let us consider $T_{1}$ as a graph; we can introduce the distance $r(x)$ of element $x$ from $a$. In particular, all elements from $\operatorname{umax}(E)$ belong to the connected component containing $a$. Let $D(i) \subseteq E$ denote the set of elements at distance at most $i$ from $a$. By what is proved above there is a composition $h^{*}$ of polynomials $h^{a b}$ for $b \in D(1)$ such that $h^{*}(D(1)) \subseteq\{a\}$. By Lemma 14 (2) every $\bar{B}$-preserving polynomial maps every $(\alpha, \beta)$-subtrace either to a singleton, or to a $(\alpha, \beta)$-subtrace 
from $T_{1}$. Therefore by induction we also get $h^{*}(D(i+1)) \subseteq D(i)$. Therefore composing several copies of $h^{*}$ collapses $a^{\prime}$ and $b^{\prime}$ and leaves $c, d$ unchanged.

We now can prove the result in Case 1 . We have proved that for any $(\alpha, \beta)$ subtrace $\left(a^{\prime}, b^{\prime}\right) \in T_{1}$ from a $\beta$-block $E$ such that $\operatorname{pr}_{12} R^{\prime} \cap(E \times\{c\}) \neq \varnothing$, a polynomial $h^{a^{\prime} b^{\prime}}$ with the required properties exists. Suppose now that $a^{\prime}, b^{\prime} \in B_{1}^{\prime}$ be any such that $\left(a^{\prime}, b^{\prime}\right) \in \beta$. Take any $c^{\prime}, d^{\prime} \in B_{2}^{\prime}$ such that $\left(a, c^{\prime}\right) \in \operatorname{pr}_{12} R^{\prime}$. By Lemmas 37 and $44(5)$ there is an idempotent $\bar{B}$-preserving polynomial $g$ such that $g\left(c^{\prime}\right)=c, g\left(d^{\prime}\right)=d$. If $g\left(a^{\prime}\right)=g\left(b^{\prime}\right)$, we are done, as $g$ may serve as $h^{a^{\prime} b^{\prime}}$. Otherwise, as before consider the relation $Q^{\dagger} \subseteq \mathbb{A}_{1}^{2} \times \mathbb{A}_{2}^{2} \times R$ generated by $\left\{\left(g\left(a^{\prime}\right), g\left(b^{\prime}\right), c, d, \mathbf{a}\right)\right\} \cup\{(x, x, y, y, \mathbf{z}) \mid \mathbf{z} \in R, \mathbf{z}[1]=x, \mathbf{z}[2]=y\}$, where $\mathbf{a}$ is an arbitrary element from $R^{\prime}$, and let $Q^{\ddagger}=\operatorname{pr}_{1234}\left(Q^{\dagger} \cap\left(B_{1}^{\prime} \times B_{1}^{\prime} \times B_{2}^{\prime} \times\right.\right.$ $\left.\left.B_{2}^{\prime} \times \bar{B}\right)\right), Q_{1}^{\prime \prime}=\operatorname{pr}_{12} Q^{\ddagger}, Q_{2}^{\prime \prime}=\operatorname{pr}_{34} Q^{\ddagger}$, and the link congruences $\mathrm{Ik}_{1}^{\prime \prime}, \mathrm{lk}_{2}^{\prime \prime}$ of $Q_{1}^{\prime \prime}, Q_{2}^{\prime \prime}$ with respect to $Q^{\ddagger}$. Recall that $C^{\prime}$ is the as-component of $B_{2}^{\prime}$ containing $c, d$. Consider relation $S=Q^{\ddagger} \cap\left(B_{1} \times B_{1} \times \operatorname{Sg}\left(C^{\prime}\right) \times \operatorname{Sg}\left(C^{\prime}\right)\right)$. Since $C^{\prime} \times C^{\prime} \subseteq$ $Q^{\prime \prime}$ by $(\mathrm{Q} 1)$, by Lemma 36 there is $\left(c^{\prime \prime}, d^{\prime \prime}\right) \in C^{\prime} \times C^{\prime}$ such that $\left(c^{\prime \prime}, d^{\prime \prime}\right)$ is asmaximal in a Ik $k_{2}^{\prime \prime}$-block and $c^{\prime \prime} \neq d^{\prime \prime}$. By the Maximality Lemma 27 (4) there is u-maximal $\left(a^{\prime \prime}, b^{\prime \prime}\right) \in Q_{1}^{\prime \prime}$ such that $\left(a^{\prime \prime}, b^{\prime \prime}, c^{\prime \prime}, d^{\prime \prime}\right) \in Q^{\prime}$. This means that for some $\bar{B}$-preserving polynomial $g^{\prime}$ it holds $g^{\prime}\left(g\left(a^{\prime}\right)\right)=a^{\prime \prime}, g^{\prime}\left(g\left(b^{\prime}\right)\right)=b^{\prime \prime}, g^{\prime}(c)=$ $c^{\prime \prime}, g^{\prime}\left(d^{\prime}\right)=d^{\prime \prime}$. By what was proved there is a polynomial $h^{a^{\prime \prime} b^{\prime \prime}}$ with $h^{a^{\prime \prime} b^{\prime \prime}}\left(a^{\prime \prime}\right)=$ $h^{a^{\prime \prime} b^{\prime \prime}}\left(b^{\prime \prime}\right)$ and $h^{a^{\prime \prime} b^{\prime \prime}}\left(c^{\prime \prime}\right)=c^{\prime \prime}, h^{a^{\prime \prime} b^{\prime \prime}}\left(d^{\prime \prime}\right)=d^{\prime \prime}$. Also, there is a $\bar{B}$-preserving polynomial $g^{\prime \prime}$ such that $g^{\prime \prime}\left(c^{\prime \prime}\right)=c, g^{\prime \prime}\left(d^{\prime \prime}\right)=d$. Finally, this all implies that $\left(a^{*}, a^{*}, c, d\right) \in Q^{\ddagger}$ for some $a^{*} \in B_{1}^{\prime}$, that is, there is a polynomial $h^{a^{\prime} b^{\prime}}=g^{\prime \prime} \circ$ $h^{a^{\prime \prime} b^{\prime \prime}} \circ g^{\prime} \circ g$ with $h^{a^{\prime} b^{\prime}}\left(a^{\prime}\right)=h^{a^{\prime} b^{\prime}}\left(b^{\prime}\right)=a^{*}$, and $h^{a^{\prime} b^{\prime}}(c)=c, h^{a^{\prime} b^{\prime}}(d)=d$.

CASE 2. For every element $c$ from a nontrivial as-component of $B_{2}^{\prime}$ and any $a \in B_{1}^{\prime}$ such that $(a, c) \in R$ element $a$ belongs to a $\beta$-block $B^{\prime}$ such that $B^{\prime} \cap$ $\operatorname{umax}\left(B_{1}^{\prime}\right)=\varnothing$ or $\left|\operatorname{umax}\left(B^{\prime} \cap B_{1}^{\prime}\right)\right|=1$.

We use the same elements $c, d \in C^{\prime}$, an as-component of $B_{2}^{\prime}$. For any $(a, b) \in$ $\left.\beta\right|_{B_{1}^{\prime}}$ choose $c^{\prime}, d^{\prime} \in B_{2}^{\prime}$ such that $\left(a, c^{\prime}\right),\left(b, d^{\prime}\right) \in \operatorname{pr}_{12} R^{\prime}$. (Recall that we are assuming $\alpha$ and $\gamma$ to be equality relations.) If $c^{\prime}=d^{\prime}$, that is, $\left(b, c^{\prime}\right) \in R$, choose $d^{\prime}$ to be an arbitrary element from $B_{2}^{\prime}$. By Lemmas 37, 44(5) and because $R$ is chained there is an idempotent $\bar{B}$-preserving polynomial $g$ such that $g\left(c^{\prime}\right)=c, g\left(d^{\prime}\right)=d$. Let $g(a)=a^{\prime}, g(b)=b^{\prime}$. Then $\left(a^{\prime}, c\right) \in R$ and $b^{\prime} \stackrel{\beta}{\equiv} a^{\prime}$. Since $g$ is $\bar{B}$-preserving, $b^{\prime} \in B_{1}^{\prime}$. We again as in Case 1 consider the relation $Q^{*} \subseteq \mathbb{A}_{1}^{2} \times \mathbb{A}_{2}^{2} \times R$ generated by $\left\{\left(a^{\prime}, b^{\prime}, c, d, \mathbf{a}\right)\right\} \cup\{(x, x, y, y, \mathbf{z}) \mid \mathbf{z} \in R, \mathbf{z}[1]=x, \mathbf{z}[2]=y\}$, where $\mathbf{a}$ is an arbitrary element from $R^{\prime}, Q^{* *}=\operatorname{pr}_{1234}\left(Q^{*} \cap\left(B_{1}^{\prime} \times B_{1}^{\prime} \times B_{2}^{\prime} \times B_{2}^{\prime} \times \bar{B}\right)\right)$, $Q_{1}^{*}=\operatorname{pr}_{12} Q^{* *}, Q_{2}^{*}=\operatorname{pr}_{34} Q^{* *}$, and the link congruences $\mathrm{Ik}_{1}^{*}, \mathrm{lk}_{2}^{*}$ of $Q_{1}^{*}, Q_{2}^{*}$ with respect to $Q^{\prime}$. Now we complete the proof as in the end of Case 1.

Finally, we use polynomials $h^{a b}$ to construct a single polynomial that collapses 
$\beta$ on $E=B^{\prime} \cap B_{1}^{\prime}$ for every $\beta$-block $B^{\prime}$. Fix $c, d$ and $h^{a b}$ for every pair $a, b \in B_{1}^{\prime}$,

$a \stackrel{\beta}{=} b$. Let $V_{1}, \ldots, V_{k}$ be the list of all such pairs, and if $V_{\ell}=\{a, b\}$ is the pair number $\ell, h^{\ell}$ denotes $h^{a b}$. Take a sequence $1=\ell_{1}, \ell_{2}, \ldots$ such that $h^{(1)}=h_{\ell_{1}}$, $V_{\ell_{2}}$ is a subset of $h^{(1)}(\mathbb{A})$, and, for $s>2, V_{\ell_{s}}$ is a subset of the range of $h^{(s-1)}=$ $h^{\ell_{s-1}} \circ \ldots \circ h^{\ell_{1}}$. Since $\left|\operatorname{Im}\left(h^{(s)}\right)\right|<\left|\operatorname{Im}\left(h^{(s-1)}\right)\right|$, there is $r$ such that $\operatorname{Im}\left(h^{(r)}\right)$ contains no pair $V_{\ell}$ for any $\ell$. Therefore setting $h(x)=h_{\ell_{r}} \circ \ldots \circ h_{\ell_{1}}(x)$ we have that $h$ collapses all the pairs $V_{\ell}$, and $h$ acts identically on $\{c, d\}$. The result follows.

\subsection{Collapsing polynomials}

We say that prime intervals $(\alpha, \beta)$ and $(\gamma, \delta)$ cannot be separated if $(\alpha, \beta)$ cannot be separated from $(\gamma, \delta)$ and $(\gamma, \delta)$ cannot be separated from $(\alpha, \beta)$. In this section we introduce and prove the existence of polynomials that collapse all prime intervals in congruence lattices of factors of a subproduct, except for a set of factors that cannot be separated from each other.

Lemma 47 (1) Let $\mathbb{A}$ be an algebra. If prime intervals $\alpha \prec \beta$ and $\gamma \prec \delta$ in $\operatorname{Con}(\mathbb{A})$ are perspective, then they cannot be separated.

(2) If $\alpha \prec \beta$ and $\gamma \prec \delta$ from $\operatorname{Con}(\mathbb{A})$ cannot be separated, then a set $U$ is $a(\alpha, \beta)$ minimal set if and only if it is a $(\gamma, \delta)$-minimal set.

(3) Let $R$ be a subdirect product of $\mathbb{A}$ and $\mathbb{B}, \alpha, \beta \in \operatorname{Con}(\mathbb{A}), \gamma, \delta \in \mathbb{B}$ such that $\alpha \prec \beta, \gamma \prec \delta$, and let $\alpha \prec \beta$ and $\gamma \prec \delta$ cannot be separated. Then for any $(\alpha, \beta)$ minimal set $U$ there is a unary idempotent polynomial $f$ such that $f(\mathbb{A})=U$ and $f(\mathbb{B})$ is a $(\gamma, \delta)$-minimal set.

Proof: (1) Follows from Lemma 16

(2) Let $f$ be a polynomial of $\mathbb{A}$ such that $f(\mathbb{A})=U$ and $f(\beta) \nsubseteq \alpha$. Since $(\alpha, \beta)$ cannot be separated from $(\gamma, \delta)$, we have $f(\delta) \nsubseteq \varnothing$ and therefore $U$ contains a $(\gamma, \delta)$-minimal set $U^{\prime}$. If $U^{\prime} \neq U$, there is a polynomial $g$ with $g \circ f(\delta) \nsubseteq \gamma$ and $g \circ f(\mathbb{A})=U^{\prime}$. In particular, $|g(U)|<|U|$, and so $g \circ f(\beta) \subseteq \alpha$; a contradiction with the assumption that $(\gamma, \delta)$ cannot be separated from $(\alpha, \beta)$.

(3) Take an idempotent polynomial $g$ of $R$ such that $g(\mathbb{B})$ is a $(\gamma, \delta)$-minimal set. Then, as $(\gamma, \delta)$ cannot be separated from $(\alpha, \beta), g(\beta) \nsubseteq \alpha$. By Lemma 14(6) there is an $(\alpha, \beta)$-minimal set $U^{\prime} \subseteq g(\mathbb{A})$. Let $g^{\prime}, h$ be polynomials of $R$ such that $g^{\prime}(U)=U^{\prime}, h\left(U^{\prime}\right)=U$ and $h(\mathbb{A})=U$, which exist by Lemma 14(1). Then $h^{\prime}=h \circ g \circ g^{\prime}$ is such that $h^{\prime}(\mathbb{A})=h^{\prime}(U)=U, h^{\prime}(\beta) \nsubseteq \propto \alpha$ and therefore $h^{\prime}(\delta) \nsubseteq$ $\gamma$. Then iterating $h^{\prime}$ sufficiently many times we get an idempotent polynomial $f$ satisfying the same properties. 
Let $R$ be a subdirect product of $\mathbb{A}_{1} \times \cdots \times \mathbb{A}_{n}$, and choose $\beta_{j} \in \operatorname{Con}\left(\mathbb{A}_{j}\right)$, $j \in[n]$. Let also $i \in[n]$, and $\alpha, \beta \in \operatorname{Con}\left(\mathbb{A}_{i}\right)$ be such that $\alpha \prec \beta \leq \beta_{i}$; let also $B_{j}$ be a $\beta_{j}$-block. We call an idempotent unary polynomial $f$ of $R \alpha \beta$-collapsing for $\bar{\beta}, \bar{B}$ if $f$ is $\bar{B}$-preserving, $f(\beta) \nsubseteq \alpha, f\left(\left.\delta\right|_{B_{j}}\right) \subseteq \gamma_{B_{j}}$ for every $\gamma, \delta \in \operatorname{Con}\left(\mathbb{A}_{j}\right)$, $j \in[n]$, with $\gamma \prec \delta \leq \beta_{j}$, and such that $(\alpha, \beta)$ can be separated from $(\gamma, \delta)$ or $(\gamma, \delta)$ can be separated from $(\alpha, \beta)$, and $|f(R)|$ is minimal possible.

Lemma 48 Let $R, \alpha, \beta$, and $\beta, j \in[n]$, be as above and $R$ chained with respect to $\bar{\beta}, \bar{B}$. Let also $R^{\prime}=R \cap \bar{B}$. Then if $\beta=\beta_{i}$ and $\operatorname{pr}_{i} R^{\prime} / \alpha$ contains a nontrivial as-component, then there exists an $\alpha \beta$-collapsing polynomial for $\bar{\beta}, \bar{B}$.

Proof: Suppose $i=1$, let $B_{1}^{\prime}=\operatorname{pr}_{1} R^{\prime}$ and $C$ be a nontrivial as-component of $B_{1}^{\prime} / \alpha$. Take a $(\alpha, \beta)$-subtrace $\{a, b\} \subseteq B_{1}^{\prime}$ such that $a^{\alpha}, b^{\alpha} \in C$. Since $R$ is chained with respect to $\bar{\beta}, \bar{B}$, by (Q1) and Lemma 44 (5) there is a $\bar{B}$-preserving idempotent polynomial $f$ of $R$ such that $f\left(\mathbb{A}_{1}\right)$ is an $(\alpha, \beta)$-minimal set and $a^{\alpha}, b^{\alpha} \in$ $f\left(\mathbb{A}_{1}\right) / \alpha$. Let polynomial $f$ be such that $f(R)$ is minimal possible. We show that $f$ is $\alpha \beta$-collapsible.

Let $j \in[n]$ and $\gamma, \delta \in \operatorname{Con}\left(\mathbb{A}_{j}\right)$ be such that $\gamma \prec \delta \leq \beta_{j}$, and $(\alpha, \beta),(\gamma, \delta)$ can be separated. Since $R$ is chained, by Lemma 46 there is an idempotent unary $\bar{B}$-preserving polynomial $f_{j \gamma \delta}$ of $R$ such that $f_{j \gamma \delta}\left(\mathbb{A}_{1}\right)$ is an $(\alpha, \beta)$-minimal set with $a^{\alpha}, b^{\alpha} \in f_{j \gamma \delta}\left(\mathbb{A}_{1}\right) / \alpha$ and $f_{j \gamma \delta}\left(\left.\delta\right|_{B_{j}}\right) \subseteq \gamma_{B_{j}}$. Then if $f\left(\left.\delta\right|_{B_{j}}\right) \nsubseteq \varnothing \gamma$, then let $g=f_{j \gamma \delta} \circ f$. We have $g(\beta) \nsubseteq \alpha$, but $g\left(\left.\delta\right|_{B_{j}}\right) \subseteq \gamma$ implying $|g(R)|<|f(R)|$, a contradiction with minimality of $f(R)$.

\subsection{Separation and minimal sets}

In this section we show a connection between the fact that two prime intervals cannot be separated, their types, and link congruences.

Lemma 49 Let $R$ be a subdirect product of $\mathbb{A}$ and $\mathbb{B}$ and let $\alpha, \beta \in \operatorname{Con}(\mathbb{A})$, $\gamma, \delta \in \operatorname{Con}(\mathbb{B})$ be such that $\alpha \prec \beta, \gamma \prec \delta$, and $(\alpha, \beta),(\gamma, \delta)$ cannot be separated. Let also $\mathrm{lk}_{1}, \mathrm{lk}_{2}$ be the link congruences of $\mathbb{A}, \mathbb{B}$, respectively. If $\operatorname{typ}(\alpha, \beta) \neq \mathbf{2}$ then $\mathrm{Ik}_{1} \wedge \beta \leq \alpha, \mathrm{lk}_{2} \wedge \delta \leq \gamma$.

Proof: Assume as usual $\alpha=\underline{0}_{\mathbb{A}}, \gamma=\underline{0}_{\mathbb{B}}$. By Lemma 47(3) there is a unary polynomial $f$ such that $f\left(\mathbb{A}_{1}\right)=U_{1}, f\left(\mathbb{A}_{2}\right)=U_{2}$ are $\left(\underline{0}_{1}, \alpha_{1}\right)$ - and $\left(\underline{0}_{2}, \alpha_{2}\right)$ minimal sets, respectively. We first study the structure of $R \cap\left(U_{1} \times U_{2}\right)$ and then show how it can be used to prove the lemma. By $N_{1}=\{0,1\}$ we denote the only trace of $U_{1}$; by $T_{1}$ we denote the tail of $U_{1}$. By Lemma 17 there is a polynomial $p(x, y)$ with $p\left(\mathbb{A}_{1}, \mathbb{A}_{1}\right)=U$ and such that $p$ is a semilattice operation 
on $N$, say, $p(0,1)=0$, and $p$ is a semilattice operation on $\{0, a\},\{1, a\}$ with $p(a, 0)=p(a, 1)=a$ for any $a \in T_{1}$. There are two cases.

CASE $1 . \operatorname{typ}(\gamma, \delta) \neq \mathbf{2}$.

Let $N_{2}=\left\{0^{\prime}, 1^{\prime}\right\}$ be the trace of $U_{2}$ and $T_{2}$ the tail of $U_{2}$. We may assume $p(x, p(x, y))=p(x, y)$. Observe first that $p$ preserves $N_{2}$. Indeed, otherwise $p(x, x)$ is not a permutation, as $p\left(0^{\prime}, 0^{\prime}\right), p\left(0^{\prime}, 1^{\prime}\right), p\left(1^{\prime}, 0^{\prime}\right), p\left(1^{\prime}, 1^{\prime}\right)$ belong to the same $\beta_{2}$-block, and if they do not belong to $N_{2}$ then they are all equal, a contradiction with the assumption that $\left(\alpha_{1}, \beta_{1}\right)$ and $\left(\alpha_{2}, \beta_{2}\right)$ cannot be separated. A binary operation on a 2-element set is either a projection, or a semilattice operation.

Suppose first that $p$ is a projection, say, the first projection on $N_{2}$. If $(\{0\} \times$ $\left.N_{2}\right) \cap R \neq \varnothing$, say, $(0, a) \in R$, then $f^{\prime}(x)=p\left(x,\left(\begin{array}{l}0 \\ a\end{array}\right)\right)$ satisfies the conditions: $f^{\prime}\left(N_{1}\right)=\{0\}$, that is, $f^{\prime}\left(\alpha_{1}\right) \subseteq \underline{0}_{1}$, and $f^{\prime}(x)=x$ on $N_{2}$; a contradiction that $(\gamma, \delta)$ cannot be separated from $(\alpha, \beta)$. If $\left(\{1\} \times N_{2}\right) \cap R \neq \varnothing$, say, $(1, a) \in R$, then $f^{\prime}(x)=p\left(\left(\begin{array}{l}1 \\ a\end{array}\right), x\right)$ satisfies the conditions: $f^{\prime}(x)=x$ on $N_{1}$, that is, $f^{\prime}\left(\beta_{1}\right) \nsubseteq \underline{0}_{1}$, and $f^{\prime}\left(N_{2}\right)=\{a\}$ on $N_{2}$; a contradiction that $(\alpha, \beta)$ cannot be separated from $(\gamma, \delta)$. Therefore, for some $a \in T_{1},\left(a, 1^{\prime}\right) \in R$. The operation $f^{\prime}=p\left(x,\left(\begin{array}{c}a \\ 1^{\prime}\end{array}\right)\right)$ is the projection on $N_{2}$ and $f^{\prime}\left(N_{1}\right)=\{a\}$; a contradiction again.

Suppose now that $p$ is a semilattice operation on $N_{2}$. Let $1^{\prime}$ be the neutral element of $p$. If $\left(a, 1^{\prime}\right) \in R$ for some $a \in T_{1}$, then $f^{\prime}(x)=p\left(x,\left(\begin{array}{c}a \\ 1^{\prime}\end{array}\right)\right)$ is the projection on $N_{2}$, and $f^{\prime}\left(N_{1}\right)=\{a\}$. If $\left(a, 1^{\prime}\right) \in R$ for no $a \in T_{1}$, then we continue as follows. If $\left(0,1^{\prime}\right)$ or $\left(1,0^{\prime}\right)$ belong to $R$, then one of the operations $p\left(x,\left(\begin{array}{c}0 \\ 1^{\prime}\end{array}\right)\right)$ and $p\left(x,\left(\begin{array}{c}1 \\ 0^{\prime}\end{array}\right)\right)$ contradicts the assumption that $i, j$ cannot be separated. Therefore $R \cap\left(U_{1} \times U_{2}\right) \subseteq\left\{\left(1,1^{\prime}\right)\right\} \cup\left(\left(\{0\} \cup T_{1}\right) \times\left(\left\{0^{\prime}\right\} \cup T_{2}\right)\right)$.

Suppose that either $\mathrm{lk}_{1} \cap \beta \neq \underline{0}_{\mathbb{A}}$ or $\mathrm{Ik}_{2} \cap \delta \neq \underline{0}_{\mathbb{B}}$, where $\mathrm{Ik}_{1}, \mathrm{lk}_{2}$ are the link congruences of $\mathbb{A}, \mathbb{B}$ with respect to $R$. Assume the latter. Then, as $\underline{0}_{\mathbb{B}} \leq \mathrm{lk}_{2}$, there is a $\left(\underline{0}_{\mathbb{B}}, \delta\right)$-trace $N$ such that $(a, b) \in \mathrm{Ik}_{2}$. This means there are $a_{1}, \ldots, a_{k} \in \mathbb{A}$ and $b_{1}, \ldots, b_{k+1} \in \mathbb{B}$ with $a=b_{1}, b=b_{k+1}$, and $\left(a_{i}, b_{i}\right),\left(a_{i}, b_{i+1}\right) \in R$. Take a polynomial $f$ of $R$ such that $U_{1}=f(\mathbb{A}), U_{2}=f(\mathbb{B})$ are $\left(\underline{0}_{\mathbb{A}}, \beta\right)$-, and $\left(\underline{0}_{\mathbb{B}}, \delta\right)$ minimal sets, respectively, and such that $U_{2}$ is a $\left(\underline{0}_{\mathbb{B}}, \delta\right)$-minimal set containing $N$ as a trace and $f(a)=a, f(b)=b$. Then, as is easily seen, $R \cap\left(U_{1} \times U_{2}\right)$ does not have the form described above. Thus, $\mathrm{Ik}_{1} \wedge \beta=\underline{0}_{\mathbb{A}}$ and $\mathrm{Ik}_{2} \wedge \delta=\underline{0}_{\mathbb{B}}$.

CASE 2. $\operatorname{typ}\left(\underline{0}_{\mathbb{B}}, \delta\right)=\mathbf{2}$.

As in Case 1 , since $p(x, x)=x$ on $U_{2}$, operation $p$ preserves every trace of $U_{2}$. 
Let $N_{2}$ be a trace in $U_{2}$. Then $N_{2}$ is polynomially equivalent to a one-dimensional vector space over $\operatorname{GF}(q)$ where $q$ is a prime power. Since $p$ is idempotent, it can be represented in the form $\gamma x+(1-\gamma) y, \gamma \in \operatorname{GF}(q)$. We may assume that $\gamma=1$. Indeed, if $\gamma=0$ then consider $p(y, x)$ instead of $p(x, y)$. Otherwise, the operation

$$
\underbrace{p(p \ldots p}_{q-1 \text { times }}(x, y), y \ldots, y)
$$

satisfies the required conditions. Now we can complete the proof as in Case 1 .

The proof of Lemma 49 also implies

Corollary 50 Let $R$ be a subdirect product of $\mathbb{A}$ and $\mathbb{B}$ and let $\alpha, \beta \in \operatorname{Con}(\mathbb{A})$, $\gamma, \delta \in \operatorname{Con}(\mathbb{B})$ be such that $\alpha \prec \beta, \gamma \prec \delta$, and $(\alpha, \beta),(\gamma, \delta)$ cannot be separated. Then $\operatorname{typ}(\alpha, \beta)=\operatorname{typ}(\gamma, \delta)$.

\section{Centralizers and decomposition of CSPs}

In this section we introduce an operator on congruence lattices similar to the centralizer in commutator theory, and study its properties and its connection to decompositions of CSPs.

\subsection{Quasi-Centralizer}

For an algebra $\mathbb{A}$, a term operation $f\left(x, y_{1}, \ldots, y_{k}\right)$, and $\mathbf{a} \in \mathbb{A}^{k}$, let $f^{\mathbf{a}}(x)=$ $f(x, \mathbf{a})$. Let $\alpha, \beta \in \operatorname{Con}(\mathbb{A}), \alpha \leq \beta$, and let $\zeta(\alpha, \beta) \subseteq \mathbb{A}^{2}$ denote the following binary relation: $(a, b) \in \zeta(\alpha, \beta)$ if an only if, for any term operation $f\left(x, y_{1}, \ldots, y_{k}\right)$, any $i \in[k]$, and any $\mathbf{a}, \mathbf{b} \in \mathbb{A}^{k}$ such that $\mathbf{a}[i]=a, \mathbf{b}[i]=b$, and $\mathbf{a}[j]=\mathbf{b}[j]$ for $j \neq i$, it holds $f^{\mathbf{a}}(\beta) \subseteq \alpha$ if and only if $f^{\mathbf{b}}(\beta) \subseteq \alpha$.

Lemma 51 For any $\alpha, \beta \in \operatorname{Con}(\mathbb{A}), \alpha \leq \beta$ :

(1) $\zeta(\alpha, \beta)$ is an equivalence relation.

(2) $\zeta(\alpha, \beta)$ is the greatest binary relation $\delta$ satisfying the condition: for any term operation $f\left(x, y_{1}, \ldots, y_{k}\right)$, and any $\mathbf{a}, \mathbf{b} \in \mathbb{A}^{k}$ such that $(\mathbf{a}[j], \mathbf{b}[j]) \in \delta$ for $j \in$ $[k]$, it holds $f^{\mathbf{a}}(\beta) \subseteq \alpha$ if and only if $f^{\mathbf{b}}(\beta) \subseteq \alpha$.

(3) $\zeta(\alpha, \beta)$ is a congruence of $\mathbb{A} .3$

\footnotetext{
${ }^{3}$ Congruence $\zeta(\alpha, \beta)$ appeared in [39], but completely inconsequentially, they did not study it at all. It is easy to see, thanks to K.Kearnes, that $\zeta(\alpha, \beta)$ is greater than the centralizer of $\alpha$ and $\beta$, but the reverse inclusion is unclear.
} 
Proof: (1) $\zeta(\alpha, \beta)$ is clearly reflexive and symmetric. Suppose $(a, b),(b, c) \in$ $\zeta(\alpha, \beta)$. Let $f\left(x, y_{1}, \ldots, y_{k}\right)$ be a term operation, $i \in[k]$, and $\mathbf{a}, \mathbf{c} \in \mathbb{A}^{k}$ such that $\mathbf{a}[i]=a, \mathbf{c}[i]=c$ and $\mathbf{a}[j]=\mathbf{c}[j]$ for $j \neq i$. Let $\mathbf{b} \in \mathbb{A}^{k}$ be such that $\mathbf{b}[i]=b$ and $\mathbf{b}[j]=\mathbf{a}[j]$ for $j \neq i$. Then $f^{\mathbf{a}}(\beta) \subseteq \alpha$ if and only if $f^{\mathbf{b}}(\beta) \subseteq \alpha$, which is if and only if $f^{\mathbf{c}}(\beta) \subseteq \alpha$.

(2) As is easily seen, $\delta$ is reflexive. Choosing $\mathbf{a}, \mathbf{b}$ that differ in only one position, we show that $\delta \subseteq \zeta(\alpha, \beta)$.

Let us show the reverse inclusion. Let $f, \mathbf{a}, \mathbf{b}$ be as in item (2) of the lemma, except $(\mathbf{a}[i], \mathbf{b}[i]) \in \zeta(\alpha, \beta)$, rather than $\delta$. Set $\mathbf{a}_{i} \in \mathbb{A}^{k}, i \in\{0, \ldots, k\}$, as follows: $\mathbf{a}_{i}[j]=\mathbf{a}[j]$ for $j \leq i$ and $\mathbf{a}_{i}[j]=\mathbf{b}[j]$ for $j>i$. Then $f^{\mathbf{a}_{i}}(\beta) \subseteq \alpha$ if and

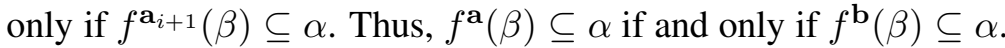

(3) By (1) $\zeta(\alpha, \beta)$ is an equivalence relation, so, we only need to show it is preserved by term operations. Let $g\left(z_{1}, \ldots, z_{m}\right)$ be a term operation and $\mathbf{a}, \mathbf{b} \in$ $\mathbb{A}^{m}$ such that $(\mathbf{a}[i], \mathbf{b}[i]) \in \zeta(\alpha, \beta)$ for $i \in[m]$. Let also $a=g(\mathbf{a})$ and $b=g(\mathbf{b})$. We show that $(a, b) \in \zeta(\alpha, \beta)$. Take a term operation $f\left(x, y_{1}, \ldots, y_{k}\right), i \in[k]$, and $\mathbf{a}^{\prime}, \mathbf{b}^{\prime} \in \mathbb{A}^{k}$ such that $\mathbf{a}^{\prime}[i]=a, \mathbf{b}^{\prime}[i]=b$, and $\left(\mathbf{a}^{\prime}[j], \mathbf{b}^{\prime}[j]\right) \in \zeta(\alpha, \beta)$ for $j \neq i$. Without loss of generality, $i=k$. Let also

$$
h\left(x, y_{1}, \ldots, y_{k-1}, z_{1}, \ldots, z_{m}\right)=f\left(x, y_{1}, \ldots, y_{k-1}, g\left(z_{1}, \ldots, z_{m}\right)\right),
$$

and $\mathbf{a}^{\prime \prime}=\left(\mathbf{a}^{\prime}[1], \ldots, \mathbf{a}^{\prime}[k-1], \mathbf{a}[1], \ldots, \mathbf{a}[m]\right), \mathbf{b}^{\prime \prime}=\left(\mathbf{b}^{\prime}[1], \ldots, \mathbf{b}^{\prime}[k-1], \mathbf{b}[1], \ldots, \mathbf{b}[m]\right)$. Then $\left(\mathbf{a}^{\prime \prime}[j], \mathbf{b}^{\prime \prime}[j]\right) \in \zeta(\alpha, \beta)$ for all $j \in[k+m-1]$. Therefore $f^{\mathbf{a}^{\prime}}(\beta)=$ $h^{\mathbf{a}^{\prime \prime}}(\beta) \subseteq \alpha$ if and only if $f^{\mathbf{b}^{\prime}}(\beta)=h^{\mathbf{b}^{\prime \prime}}(\beta) \subseteq \alpha$.

The congruence $\zeta(\alpha, \beta)$ will be called the quasi-centralizer of $\alpha, \beta$. Next we prove several properties of quasi-centralizer similar to some extent to the properties of the regular centralizer. The following statement is one of the key ingredients of the algorithm.

Proposition 52 If $\zeta(\alpha, \beta) \geq \beta$, then $(\alpha, \beta)$ has type $\mathbf{2}$, and for any $\beta$-blocks $B, C$ such that $B \leq C$ in $\mathbb{A} / \beta$ (that is $B C$ is a thin semilattice edge in $\mathbb{A} / \beta$ ) and they belong to the same $\zeta(\alpha, \beta)$-block, there is an injective mapping $\sigma: B / \alpha \rightarrow C / \alpha$ such that for any $a \in B / \alpha, a \leq \sigma(a)$ and $a \not \leq b$ for any other $b \in C$.

Proof: Clearly, we may assume $\alpha=\underline{0}$. Suppose that $\zeta(\alpha, \beta) \geq \beta$, and suppose first that $\operatorname{typ}(\underline{0}, \beta) \neq \mathbf{2}$. Take any $(\underline{0}, \beta)$-minimal set $U$, its only trace $N$, and a pseudo-meet operation $p$ on $U$. Then the polynomial $p(x, 0)$ does not collapse $\beta$, as $f(0,0)=0, f(1,0)=1$, while the polynomial $p(x, 1)$ does, a contradiction with the assumption $\zeta(\alpha, \beta) \geq \beta$.

Suppose now that $\operatorname{typ}(\underline{0}, \beta)=\mathbf{2}$. Then by Corollary $\underline{39}$ (1) for any $a, b \in \mathbb{A}$ with $a \stackrel{\beta}{=} b$, there is a $(\underline{0}, \beta)$-minimal set $U$ such that $a, b \in U$. 
Let $B, C$ be $\beta$-blocks and $B \leq C$ in $\mathbb{A} / \beta$. By Lemma 22(1) for any $a \in \mathbb{A}$ there is $b \in C$ with $a \leq b$. Suppose the statement of the proposition is not true. Then there are two possibilities.

1. For some $a \in B$ and $b, c \in C, a \leq b, a \leq c$. Let $f$ be a polynomial such that $U=f(\mathbb{A})$ is a $(\underline{0}, \beta)$-minimal set and $b, c \in U$. Consider $g_{1}(x)=a \cdot f(x)$ and $g_{2}(x)=b \cdot f(x)$. Clearly, $g_{1}(b)=b, g_{1}(c)=c$, so $g_{1}(\beta) \nsubseteq \underline{0}$. On the other hand, $g_{2}(b)=b=g_{2}(c)$, that is, $g_{2}(\beta) \subseteq \underline{0}$, as $\left|g_{2}(\mathbb{A})\right| \leq|U|$, a contradiction with the assumption $(a, b) \in \zeta(\underline{0}, \beta)$.

2. For some $a \in C$ and $b, c \in B, b \leq a, c \leq a$. Let $f$ be a polynomial such that $U=f(\mathbb{A})$ is a $(\underline{0}, \beta)$-minimal set and $b, c \in U$. Consider $g_{1}(x)=f(x) \cdot a$ and $g_{2}(x)=f(x) \cdot b$. Clearly, $g_{1}(b)=g_{1}(c)=a$, so $g_{1}(\beta) \subseteq \underline{0}$, as $\left|g_{1}(\mathbb{A})\right| \leq|U|$. On the other hand, $g_{2}(b)=b, g_{2}(c)=c$, that is, $g_{2}(\beta) \nsubseteq \underline{0}$ a contradiction again.

Corollary 53 Let $\zeta(\alpha, \beta)=\underline{1}_{\mathbb{A}}, a, b, c \in \mathbb{A}$ and $b \stackrel{\beta}{\equiv} c$. Then $a b \stackrel{\alpha}{=} a c$.

Proof: We have $a b \stackrel{\beta}{\equiv} a c$ and $a \leq a b, a \leq a c$. By Proposition $52 a b \stackrel{\alpha}{\equiv} a c$.

Remark 54 Recently, Payne [56] designed a polynomial time algorithm for the following class of algebras: Every algebra $\mathbb{A}$ from this class has a congruence $\alpha$ such that $\mathbb{A} / \alpha$ is a semilattice, and the interactions between $\alpha$-blocks satisfy a certain condition. It seems that Lemma 52 is similar to what this condition can provide.

An injective mapping between $\beta$-blocks $B, C$ inside a $\zeta(\alpha, \beta)$-block can also be established whenever $B C$ is any thin edge in $\mathbb{A} / \beta$, as the following lemma shows.

Lemma 55 Let $\alpha, \beta \in \operatorname{Con}(\mathbb{A})$ such that $\alpha \prec \beta \leq \zeta=\zeta(\alpha, \beta)$ and let $B, C$ be $\beta$-blocks from the same $\zeta$-block such that $B C$ is a thin edge in $\mathbb{A} / \beta$. For any $b \in B, c \in C$ such that $b c$ is a thin edge the polynomial $f(x)=x \cdot c$ if $b \leq c$, $f(x)=t_{b c}(x, c)$ if bc is majority, and $f(x)=h_{b c}(x, b, c)$ if bc is affine, where $t_{a b}, h_{a b}$ are the operations from Lemma 24 is an injective mapping from $B / \alpha$ to $C / \alpha$.

Proof: We can assume that $\alpha$ is the equality relation. Suppose $f\left(a_{1}\right)=f\left(a_{2}\right)$ for some $a_{1}, a_{2} \in B$. Since $\operatorname{typ}(\alpha, \beta)=\mathbf{2}$, by Corollary 39 (1) every pair of elements of $B$ is an $(\alpha, \beta)$-subtrace. Let $f^{\prime}$ be an idempotent unary polynomial such that $f^{\prime}\left(a_{1}\right)=a_{1}, f^{\prime}\left(a_{2}\right)=a_{2}$, and $f^{\prime}(\mathbb{A})$ is an $(\alpha, \beta)$-minimal set. 
If $b \leq c$, let $g(x, y)=f^{\prime}(x) \cdot y$. Then $g^{c}=g(x, c)=f(x)$ on $\left\{a_{1}, a_{2}\right\}$, that is, $g^{c}\left(a_{1}\right)=g^{c}\left(a_{2}\right)$ implying $g^{c}(\beta) \subseteq \alpha$. On the other hand, $g^{b}(x)=f^{\prime}(x)$ on $\left\{a_{1}, a_{2}\right\}$ implying $g^{b}(\beta) \nsubseteq \alpha$, a contradiction with the assumption $b \stackrel{\zeta}{\equiv} c$.

If $b c$ is a thin majority edge, set $g(x, y)=t_{b c}\left(f^{\prime}(x), y\right)$. Then $g^{c}\left(a_{1}\right)=$ $f\left(a_{1}\right)=f\left(a_{2}\right)=g^{c}\left(a_{2}\right)$, and so $g^{c}(\beta) \subseteq \alpha$. On the other hand, since $B / \alpha$ is a module, $a_{1} b, a_{2} b$ are affine edges and $\alpha$ witnesses that. Therefore $g^{b}\left(a_{1}\right)=a_{1}$ and $g^{b}\left(a_{2}\right)=a_{2}$, implying $g^{b}(\beta) \nsubseteq \alpha$, and we have a contradiction again.

Finally, if $b c$ is a thin affine edge, we consider the polynomials $g(x, y, z)=$ $h_{b c}\left(f^{\prime}(x), y, z\right)$ and $g^{b c}(x)=g(x, b, c), g^{a_{1} a_{1}}(x)=g\left(x, a_{1}, a_{1}\right)$. Again, $g^{b c}\left(a_{1}\right)=$ $f\left(a_{1}\right)=f\left(a_{2}\right)=g^{b c}\left(a_{2}\right)$, while

$$
g^{a_{1} a_{1}}\left(a_{1}\right)=h_{b c}\left(f^{\prime}\left(a_{1}\right), a_{1}, a_{1}\right)=a_{1} \neq h_{b c}\left(f^{\prime}\left(a_{2}\right), a_{1}, a_{1}\right)=g^{a_{1} a_{1}}\left(a_{2}\right),
$$

since by Lemma $24 h_{b c}\left(x, a_{1}, a_{1}\right)$ is a permutation. This implies that $g^{b c}(\beta) \subseteq \alpha$ and $g^{a_{1} a_{1}}(\beta) \nsubseteq \alpha$, a contradiction.

Lemma 56 Let $\alpha, \beta \in \operatorname{Con}(\mathbb{A})$ be such that $\alpha \prec \beta$ and $\operatorname{typ}(\alpha, \beta)=\mathbf{2}$, and $\zeta=\zeta(\alpha, \beta)$. Then for any $\beta$-blocks $B_{1}, B_{2}$ that belong to the same $\zeta$-block $C$ and such that $B_{1} \sqsubseteq$ asm $B_{2}$ and $B_{2} \sqsubseteq$ asm $B_{1}$ in $C / \beta,\left|B_{1} / \alpha\right|=\left|B_{2} / \alpha\right|$.

Proof: Since there is an asm-path from $B_{1}$ to $B_{2}$ and back, the result follows from Lemma 55 ,

Let $\mathbb{A}$ be an algebra and $\alpha, \beta \in \operatorname{Con}(\mathbb{A}), \alpha \prec \beta$. Element $a \in \mathbb{A}$ is said to be $\alpha \beta$-minimal if it belongs to an $(\alpha, \beta)$-trace. Let $\mathrm{Z}_{\mathbb{A}}(\alpha, \beta)$ denote the set of all $\alpha \beta$-minimal elements of $\mathbb{A}$. By Lemma 14 (5) $\mathrm{Z}_{\mathbb{A}}(\alpha, \beta)$ intersects every $\alpha$-block from a nontrivial $\beta$-block. The following lemma shows that $Z_{\mathbb{A}}(\alpha, \beta)$ can be much larger than that. Due to the way we will use it in the future the statement of the lemma is not quite straightforward.

Lemma 57 Let $\alpha, \beta, \gamma, \delta \in \operatorname{Con}(\mathbb{A})$ be such that $\gamma \prec \delta \leq \beta, \alpha \prec \beta$, intervals $(\alpha, \beta),(\gamma, \delta)$ cannot be separated, and $\operatorname{typ}(\alpha, \beta)=2$; let $B$ be a $\beta$-block. If $a \in Z_{\mathbb{A}}(\gamma, \delta) \cap B$ then for any $b \in B$ such that $a \sqsubseteq$ asm $b$ in $B, b \in \mathbf{Z}_{\mathbb{A}}(\gamma, \delta)$.

In particular, umax $(B) \subseteq \mathrm{Z}_{\mathbb{A}}(\gamma, \delta)$.

Moreover, if $a \stackrel{\alpha}{\equiv} b, a \sqsubseteq a s m b$ in $a^{\alpha}$, and $f$ is a polynomial such that $f(a)=$ $a, f(\mathbb{A})$ is an $(\alpha, \beta)$-minimal set, and $N$ its trace with $a \in N$, then there is a polynomial $g$ such that $g(b)=b, g(\mathbb{A})$ is an $(\alpha, \beta)$-minimal set, $N^{\prime}$ is its trace containing $b$ and $N^{\prime} / \alpha=N / \alpha$.

Proof: Let $f$ be an idempotent unary polynomial of $\mathbb{A}$ such that $a \in N$, a trace in $U=f(\mathbb{A})$, a $(\gamma, \delta)$-minimal set. Note that $f(B) \subseteq B$ and $f(\beta) \nsubseteq \alpha$. It suffices to consider the case when $a b$ is a thin edge. 
Depending on the type of the edge $a b$ we set $f^{\prime}(x)=f(x) \cdot b, f^{\prime}(x)=$ $t_{a b}(f(x), b)$, or $f^{\prime}(x)=h_{a b}(f(x), a, b)$, if $a b$ is semilattice, majority or affine, respectively. Note also that by Lemma $24 f^{\prime}(a)=b$, and therefore if $f^{\prime}(\delta) \nsubseteq \gamma$ we have $f^{\prime}(\mathbb{A})$ is a $(\gamma, \delta)$-minimal set, and $b$ belongs to it.

Since $(\alpha, \beta)$ and $(\gamma, \delta)$ cannot be separated, by Lemma 47(2) $U$ is an $(\alpha, \beta)$ minimal set. Hence, there are $a_{1}, a_{2} \in B / \alpha$ such that $a_{1} \neq a_{2}$ and $f\left(a_{1}\right)=$ $a_{1}, f\left(a_{2}\right)=a_{2}$. Since $a_{1} a_{2}$ is an affine edge in $\mathbb{B} / \alpha$, depending on the type of $a b$ we have:

- if $a \leq b$, then $f^{\prime}\left(a_{i}\right)=a_{i} \cdot b^{\alpha}=a_{i}$ for $i=1,2$;

- if $a b$ is majority, then $f^{\prime}\left(a_{i}\right)=t_{a b}\left(a_{i}, b^{\alpha}\right)=a_{i}$, as $a_{i} \stackrel{\beta / \alpha}{\equiv} b^{\alpha}$ for $i=1,2$ and by Lemma 24(1);

- if $a b$ is affine, then by Lemma 24 (2) $h_{a b}\left(x, a^{\alpha}, b^{\alpha}\right)$ is a permutation on $B / \alpha$, in particular, $f^{\prime}\left(a_{1}\right) \neq f^{\prime}\left(a_{2}\right)$.

In either case we obtain $f^{\prime}(\beta) \nsubseteq \varnothing \alpha$, implying $f^{\prime}(\delta) \nsubseteq \varnothing$.

For the last claim of the lemma it suffices to notice that if $a \stackrel{\alpha}{\equiv} b$ then $f^{\prime}(x) \stackrel{\alpha}{\equiv}$ $f(x)$ for $x \in B$.

\subsection{Decomposition of CSPs}

In this section we show that if intervals in congruence lattices of domains in a CSP instance cannot be separated, they induce certain decomposition of the instance or its subinstances. The components of this decomposition are instances over smaller domains, which are, actually, blocks of the corresponding quasi-centralizers.

Let $R$ be a subdirect product of $\mathbb{A}_{1} \times \cdots \times \mathbb{A}_{n}, i, j \in[n]$, and $\alpha_{i} \in \operatorname{Con}\left(\mathbb{A}_{i}\right)$, $\alpha_{j} \in \operatorname{Con}\left(\mathbb{A}_{j}\right)$. The coordinate positions $i, j$ are said to be $\alpha_{i} \alpha_{j}$-aligned in $R$ if, for any $(a, c),(b, d) \in \operatorname{pr}_{i j} R,(a, b) \in \alpha_{i}$ if and only if $(c, d) \in \alpha_{j}$. Or in other words, the link congruences of $\mathbb{A}_{i}, \mathbb{A}_{j}$ with respect to $\operatorname{pr}_{i j} R$ are no greater than $\alpha_{i}, \alpha_{j}$, respectively.

Lemma 58 Let $R$ be a subdirect product of $\mathbb{A}_{1} \times \mathbb{A}_{2}, \alpha_{i}, \beta_{i} \in \operatorname{Con}\left(\mathbb{A}_{i}\right), \alpha_{i} \prec \beta_{i}$, for $i=1,2$. If $\left(\alpha_{1}, \beta_{1}\right)$ and $\left(\alpha_{2}, \beta_{2}\right)$ cannot be separated from each other, then the coordinate positions 1,2 are $\zeta\left(\alpha_{1}, \beta_{1}\right) \zeta\left(\alpha_{2}, \beta_{2}\right)$-aligned in $R$.

Proof: Let us assume the contrary, that is, without loss of generality there are $a, b \in \mathbb{A}_{1}$ and $c, d \in \mathbb{A}_{2}$ with $(a, c),(b, d) \in R,(a, b) \in \zeta\left(\alpha_{1}, \beta_{1}\right)$, but $(c, d) \notin$ $\zeta\left(\alpha_{2}, \beta_{2}\right)$. Therefore there is $g\left(x, y_{1}, \ldots, y_{k}\right)$, a term operation of $\mathbb{A}_{2}, i \in[k]$, and $\mathbf{c}, \mathbf{d} \in \mathbb{A}_{2}^{k}$ with $\mathbf{c}[i]=c, \mathbf{d}[i]=d$ and $\mathbf{c}[j]=\mathbf{d}[j]$ for $j \neq i$, such that $g^{\mathbf{c}}\left(\beta_{2}\right) \subseteq \alpha_{2}$ but $g^{\mathbf{d}}\left(\beta_{2}\right) \nsubseteq \alpha_{2}$, or the other way round. Extend $g$ to a term operation $g$ of $R$, and choose $\mathbf{a}, \mathbf{b} \in \mathbb{A}_{1}^{k}$ such that $\mathbf{a}[i]=a, \mathbf{b}[i]=b, \mathbf{a}[j]=\mathbf{b}[j]$ for $j \neq i$, and $(\mathbf{a}[j], \mathbf{c}[j]),(\mathbf{b}[j], \mathbf{d}[j]) \in R$ for $j \in[k]$. Then $g^{\mathbf{a}}\left(\beta_{1}\right) \subseteq \alpha_{1}$ if and only if 
$g^{\mathbf{b}}\left(\beta_{1}\right) \subseteq \alpha_{1}$. Therefore, there is a polynomial of $R$ that separates $\left(\alpha_{1}, \beta_{1}\right)$ from $\left(\alpha_{2}, \beta_{2}\right)$ or the other way round, a contradiction.

Let $\mathcal{P}=(V, \mathcal{C})$ be a $(2,3)$-minimal instance, in particular, for every $X \subseteq V$, $|X|=2$, it contains a constraint $C^{X}=\left\langle X, R^{X}\right\rangle$. Let $w_{1}, w_{2}, \in V$. We say that $w_{1}, w_{2}$ are $\alpha_{1} \alpha_{2}$-aligned in $\mathcal{P}$, where $\alpha_{1} \in \operatorname{Con}\left(\mathbb{A}_{w_{1}}\right), \alpha_{2} \in \operatorname{Con}\left(\mathbb{A}_{w_{2}}\right)$, if they are $\alpha_{1} \alpha_{2}$-aligned in $R^{w_{1} w_{2}}$. For $\alpha_{v} \in \operatorname{Con}\left(\mathbb{A}_{v}\right), v \in V$, instance $\mathcal{P}$ is said to be $\bar{\alpha}$-aligned if every $w_{1}, w_{2}$ are $\alpha_{w_{1}} \alpha_{w_{2}}$-aligned. This means that there are one-toone mappings $\varphi_{w_{1} w_{2}}: \mathbb{A}_{w_{1}} / \alpha_{w_{1}} \rightarrow \mathbb{A}_{w_{1}} / \alpha_{w_{1}}$ such that whenever $(a, b) \in R^{w_{1} w_{2}}$, $b^{\alpha_{w_{2}}}=\varphi_{w_{1} w_{2}}\left(a^{w_{1}}\right)$. Observe that since $\mathcal{P}$ is $(2,3)$-minimal, these mappings are consistent, that is, for any $u, v, w \in V, \varphi_{v w} \circ \varphi_{u v}=\varphi_{u w}$. Therefore $\mathcal{P}$ can be represented as a disjoint union of instances $\mathcal{P}_{1}, \ldots, \mathcal{P}_{k}$, where $k$ is the number of $\alpha_{v}$-blocks for any $v \in V$ and the domain of $v \in V$ of $\mathcal{P}_{i}$ is the $i$-th $\alpha_{v}$-block.

Let again $\mathcal{P}=(V, \mathcal{C})$ be a $(2,3)$-minimal instance and let $\bar{\beta}, \beta_{v} \in \operatorname{Con}\left(\mathbb{A}_{v}\right), v \in$ $V$, be a collection of congruences. Let $\mathcal{W}^{\mathcal{P}}(\bar{\beta})$ denote the set of triples $(v, \alpha, \beta)$ such that $v \in V, \alpha, \beta \in \operatorname{Con}\left(\mathbb{A}_{v}\right)$, and $\alpha \prec \beta \leq \beta_{v}$. Also, $\mathcal{W}^{\mathcal{P}}$ denotes $\mathcal{W}^{\mathcal{P}}(\bar{\beta})$ when $\beta_{v}=\underline{1}_{v}$ for all $v \in V$. We will omit the superscript $\mathcal{P}$ whenever it is clear from the context. For every $(v, \alpha, \beta) \in \mathcal{W}(\bar{\beta})$, let $Z$ denote the set of triples $(w, \gamma, \delta) \in \mathcal{W}(\bar{\beta})$ such that $(\alpha, \beta)$ and $(\gamma, \delta)$ cannot be separated in $R^{v w}$. Slightly abusing the terminology we will also say that $(\alpha, \beta)$ and $(\gamma, \delta)$ cannot be separated in $\mathcal{P}$. Then let $W_{v, \alpha \beta, \bar{\beta}}=\left\{w \in V \mid(w, \gamma, \delta) \in Z\right.$ for some $\left.\gamma, \delta \in \operatorname{Con}\left(\mathbb{A}_{w}\right)\right\}$. We will omit the subscript $\bar{\beta}$ whenever possible. The following statement is an easy corollary of Lemma 58 .

Theorem 59 Let $\mathcal{P}=(V, \mathcal{C})$ be a (2,3)-minimal instance and $(v, \alpha, \beta) \in \mathcal{W}$. For $w \in W_{v, \alpha \beta, \bar{\beta}}$, where $\beta_{v}=\underline{1}_{v}$ for $v \in V$, let $(w, \gamma, \delta) \in \mathcal{W}$ be such that $(\alpha, \beta)$ and $(\gamma, \delta)$ cannot be separated and $\zeta_{w}=\zeta(\gamma, \delta)$. Then $\mathcal{P}_{W_{v, \alpha \beta, \bar{\beta}}}$ is $\bar{\zeta}$-aligned.

\section{The Congruence Lemma}

This section contains several technical results that will be used when proving of the soundness of the algorithm. The main result is the Congruence Lemma 64 Lemmas 62 and 63 are auxiliary and only used in the proof of the Congruence Lemma 64. We start with introducing two closure properties of algebras and their subdirect products, this time under certain polynomials.

We say that a set $A$ is as-closed in algebra $\mathbb{B}, A \subseteq \mathbb{B}$, if $A \cap \operatorname{umax}(\mathbb{B}) \neq \varnothing$ and, for every $a, b \in \mathbb{B}$ such that $a \sqsubseteq$ as $b$ in $\mathbb{B}$ and $a \in A \cap \operatorname{umax}(\mathbb{B})$, element $b$ also belongs to $A$.

Let $R$ be a subdirect product of $\mathbb{A}_{1}, \ldots, \mathbb{A}_{n}$ and $Q$ a subalgebra of $R$. We say that $Q$ is polynomially closed in $R$ if for any polynomial $f$ of $R$ the following 
condition holds: for any $\mathbf{a}, \mathbf{b} \in \operatorname{umax}(Q)$ such that $f(\mathbf{a})=\mathbf{a}$ and for any $\mathbf{c} \in$ $\mathrm{Sg}(\mathbf{a}, f(\mathbf{b}))$ such that $\mathbf{a} \sqsubseteq_{a s} \mathbf{c}$ in $\operatorname{Sg}(\mathbf{a}, f(\mathbf{b}))$, the tuple $\mathbf{c}$ belongs to $Q$.

Remark 60 Polynomially closed subalgebras of Mal'tsev algebras are congruence blocks. In the general case the structure of polynomially closed subalgebras is more intricate. The intuition (although not entirely correct) is that if for some block $B$ of a congruence $\beta$ and a congruence $\alpha$ with $\alpha \prec \beta$ the set $B / \alpha$ contains several as-components, a polynomially closed subalgebra contains some of them and has empty intersection with the rest. However, since this is true only for factor sets, and we do not even consider non-as-maximal elements, the actual structure is more 'fractal'.

The following lemma follows from the definitions, Lemma22, and the fact that congruences are invariant under polynomials.

Lemma 61 (1) Let $R$ be a subdirect product of $\mathbb{A}_{1}, \ldots, \mathbb{A}_{n}$ and $Q_{1}, Q_{2}$ relations polynomially closed in $R$, and $Q_{1} \cap Q_{2} \cap \operatorname{umax}(Q) \neq \varnothing$. Then $Q_{1} \cap Q_{2}$ is polynomially closed in $R$.

In particular, let $\beta_{i} \in \operatorname{Con}\left(\mathbb{A}_{i}\right)$ and $B_{i}$ a u-maximal $\beta_{i}$-block. Then $Q_{1} \cap \bar{B}$ is polynomially closed in $R$.

If $Q_{1}, Q_{2}$ are as-closed in $R$, then $Q_{1} \cap Q_{2}$ is as-closed in $R$.

(2) Let $Q_{i}$ be polynomially closed in $R_{i}, i \in[k]$, and let $R, Q$ be pp-defined through $R_{1}, \ldots, R_{k}$ and $Q_{1}, \ldots, Q_{k}$, respectively, by the same pp-formula $\Phi$; that is, $R=$ $\Phi\left(R_{1}, \ldots, R_{k}\right)$ and $Q=\Phi\left(Q_{1}, \ldots, Q_{k}\right)$. Let also for every atom $R_{i}\left(x_{1}, \ldots, x_{\ell}\right)$ and any $\mathbf{a} \in \operatorname{umax}\left(R_{i}\right)$ there is $\mathbf{b} \in R$ with $\operatorname{pr}_{\left\{x_{1}, \ldots, x_{\ell}\right\}} \mathbf{b}=\mathbf{a}$, and also umax $(Q) \cap$ $\operatorname{umax}(R) \neq \varnothing$. Then $Q$ is polynomially closed in $R$.

If $Q_{i}$ is as-closed in $R_{i}$ then $Q$ is as-closed in $R$.

(3) Let $R$ be a subdirect product of $\mathbb{A}_{1}, \ldots, \mathbb{A}_{n}, \beta_{i} \in \operatorname{Con}\left(\mathbb{A}_{i}\right), i \in[n]$, and let $Q$ be polynomially closed in $R$. Then $Q / \bar{\beta}$ is polynomially closed in $R$.

If $Q$ is as-closed in $R$ then $Q / \bar{\beta}$ is as-closed in $R / \bar{\beta}$.

The first two lemmas in the rest of this section study the structure of binary relations that have in their domains a pair of prime intervals of type $\mathbf{2}$ that cannot be separated. They show that if we restrict ourselves to blocks of the link congruences then this structure is very uniform. The third lemma, Lemma 64 (the Congruence Lemma), is an important technical result. To explain what it amounts to saying consider this: let $Q \subseteq \mathbb{A}^{\prime} \times \mathbb{B}^{\prime}$ be a subdirect product and the link congruence of $\mathbb{A}^{\prime}$ is the equality relation. Then, clearly, $Q$ is the graph of a mapping $\sigma: \mathbb{B}^{\prime} \rightarrow \mathbb{A}^{\prime}$, and the kernel of this mapping is the link congruence $\eta$ of $\mathbb{B}^{\prime}$ with respect to $Q$. Suppose now that $Q$ is a subalgebra of $R$, a subdirect product of $\mathbb{A} \times \mathbb{B}$ such that 
$\mathbb{A}^{\prime}$ is a subalgebra of $\mathbb{A}$ and $\mathbb{B}^{\prime}$ is a subalgebra of $\mathbb{B}$. Then the restriction of the link congruence of $\mathbb{A}$ with respect to $R$ to $\mathbb{A}^{\prime}$ does not have to be the equality relation, and similarly the restriction of the link congruence of $\mathbb{B}$ to $\mathbb{B}^{\prime}$ does not have to be $\eta$. Most importantly, the restriction of $\mathrm{Cg}(\eta)$, the congruence of $\mathbb{B}$ generated by $\eta$, to $\mathbb{B}^{\prime}$ does not have to be $\eta$. The Congruence Lemma 64 shows, however, that this is exactly what happens when $Q$ and $\mathbb{A}^{\prime}, \mathbb{B}^{\prime}$ satisfy some additional conditions, such as being chained and polynomially closed.

In the next two lemmas let $R$ be a subdirect product of $\mathbb{A}_{1} \times \mathbb{A}_{2}, \beta_{1}, \beta_{2}$ congruences of $\mathbb{A}_{1}, \mathbb{A}_{2}$ and $B_{1}, B_{2} \beta_{1}$ - and $\beta_{2}$-blocks, respectively; $R$ is chained with respect to $\left(\beta_{1}, \beta_{2}\right),\left(B_{1}, B_{2}\right)$ and $R^{*}=R \cap\left(B_{1} \times B_{2}\right), B_{1}^{*}=\operatorname{pr}_{1} R^{*}, B_{2}^{*}=$ $\operatorname{pr}_{2} R^{*}$. Let $\alpha, \beta \in \operatorname{Con}\left(\mathbb{A}_{1}\right), \gamma, \delta \in \operatorname{Con}\left(\mathbb{A}_{2}\right)$ be such that $\alpha \prec \beta \leq \beta_{1}$, $\gamma \prec \delta \leq \beta_{2}$, typ $(\alpha, \beta)=\operatorname{typ}(\gamma, \delta)=\mathbf{2}$, and $(\alpha, \beta),(\gamma, \delta)$ cannot be separated. Let also $\zeta_{1}=\left.\zeta(\alpha, \beta)\right|_{B_{1}^{*}}, \zeta_{2}=\left.\zeta(\gamma, \delta)\right|_{B_{2}^{*}}$ and $\mathrm{Ik}_{1}^{*}$, Ik $\mathrm{k}_{2}^{*}$ the link congruences of $B_{1}^{*}, B_{2}^{*}$, respectively, with respect to $R^{*}$. Let $F, G$ be $\zeta_{1^{-}}, \zeta_{2}$-blocks such that $R^{*} \cap(F \times G) \neq \varnothing$ and $F, G$ contain nontrivial $\beta$ - and $\delta$-blocks $A, B$, respectively (that is, $|A / \alpha|,|B / \gamma|>1$ ).

By Lemma 56 all the $\beta$-blocks $A^{\prime} \in F / \beta, A \sqsubseteq_{\text {asm }} A^{\prime}$ in $F$ (respectively, all $\delta$-blocks $B^{\prime} \in G / \delta, B \sqsubseteq_{\text {asm }} B^{\prime}$ in $G$ ) are also nontrivial. Note that by Lemma 58 $\mathrm{Ik}_{1}^{*} \leq \zeta_{1}$ and $\mathrm{Ik}_{2}^{*} \leq \zeta_{2}$. Let also $D \subseteq F, E \subseteq G$ be blocks of $\mathrm{Ik}_{1}^{*}, \mathrm{Ik}_{2}^{*}$ such that $R^{*} \cap(D \times E) \neq \varnothing$.

The first lemma claims that for the link congruence $\mathrm{Ik}_{2}^{*}$ there are only two options: either it is a subset of $\gamma$ on the $\zeta_{2}$-block $G$, or it contains $\delta / \gamma$ on umax $(G)$.

Lemma 62 Suppose that $B \cap E \neq \varnothing$ and that $\mathrm{Ik}_{2}^{*}$ is nontrivial on the $\delta$-block $B$, that is, there are distinct $a, b \in\left(B \cap B_{2}^{*}\right) / \gamma$ with $(a, b) \in \mathrm{Ik}_{2}^{*}$, or equivalently $\mathrm{Ik}_{2}^{*} \wedge \delta$ is not a subset of $\gamma$ on $B \cap B_{2}^{*}$. Then

(1) if $G \cap \operatorname{umax}\left(B_{2}^{*}\right) \neq \varnothing$ then $\left.\delta\right|_{\mathrm{umax}(G)} \leq \mathrm{Ik}_{2}^{*} \vee \gamma_{B_{2}^{*}}$; and

(2) any $B^{\prime} \in G / \delta$ with $B \sqsubseteq$ asm $B^{\prime}$ in $G / \delta$ is nontrivial, that is, $\left|B^{\prime} / \gamma\right|>1$. In particular, umax $(D), \operatorname{umax}(E)$ and $\operatorname{umax}(F), \operatorname{umax}(G)$ do not intersect any trivial $\beta$ - and $\delta$-blocks, respectively.

Proof: Since $\mathrm{Ik}_{1}^{*} \leq \zeta_{1}$ and $\mathrm{Ik}_{2}^{*} \leq \zeta_{2}$, (2) follows by Lemma 56, Also, as $\mathrm{Ik}_{2}^{*}$ is nontrivial on a $\delta$-block, we obtain (1) by Lemma 45 .

The second lemma amounts to saying that if $\mathrm{Ik}_{2}^{*}$ is nontrivial on $G$ then it not only contains $\delta$ (modulo $\gamma$ ), but also that if an element of $F$ is related by $R$ to some element of a $\delta$-block $B$, it is also related to the entire $B$ (again, modulo $\gamma$ ).

Lemma 63 Suppose $\left.\delta\right|_{\mathrm{umax}(G)} \leq \mid \mathrm{k}_{2}^{*} \vee \gamma_{B_{2}^{*}}$ and sets $A, B$ satisfy one of the following two conditions:

(1) let $A \subseteq F, B \subseteq G$ be $\beta$ - and $\delta$-blocks, respectively, such that $(A, B) \in$ 
$\operatorname{umax}((R \cap(F \times G)) / \beta \times \delta)$, or

(2) let $A, B$ be $\beta$-, and $\delta$-blocks, respectively, and such that $A^{\prime}=A \cap D \neq \varnothing$ and $\left(A^{\prime}, B\right) \in \operatorname{umax}((R \cap(D \times G)) / \beta \times \delta)$.

Then either $R \cap(A \times B)=\varnothing$, or for any $c \in A$ with $B \cap R[c] \neq \varnothing$ we have $\{c\} \times B / \gamma \subseteq R / \gamma$.

Note that in condition (2) $D$ does not have to contain u-maximal elements of $F$, and similarly $E$ does not have to contain u-maximal elements of $G$. Thus, (1) is not necessarily a more general condition.

Proof: We prove the lemma for condition (2), that is, when $\left(A^{\prime}, B\right) \in \operatorname{umax}((R \cap$ $(D \times G)) / \beta \times \delta)$. It will be clear that case (1) follows from the same argument.

We assume $\gamma=\underline{0}_{2}$. Since $B$ is a module, it is as-connected. Therefore if some element of $B$ belongs to an as-component of $E$, the whole set $B$ is contained in that as-component. By the Rectangularity Corollary 34, this means that if $R \cap(\{c\} \times$ $B) \neq \varnothing$ for $c \in \operatorname{amax}(D)$, then $\{c\} \times B \subseteq R$, and the result for $B$ follows.

Now we show that if $\{c\} \times B \subseteq R$ for some $c \in D$ and $B \in E / \delta$ then $\{d\} \times b^{\prime \delta} \subseteq R$ for any $d \in D$ and $b^{\prime} \in E$ such that $(c, b)\left(d, b^{\prime}\right)$ is a thin edge in $R \cap(D \times E)$ for some $b \in B$. As is easily seen this implies the result. There are 3 possible cases.

CASE $1 . b^{\prime \delta}=B$, that is $c d$ is a thin edge and $\left(d, b^{\prime}\right) \in R, b^{\prime} \in B$. Then $\{d\} \times B \subseteq R$.

This case follows from Lemma 32 .

CASE 2. $c=d$, that is, $B B^{\prime}$ is a thin edge in $\mathbb{A}_{2} / \delta$ where $B^{\prime}=b^{\prime \delta}$ and $\left(c, b^{\prime}\right) \in R$.

Let $f(x)$ be the unary polynomial of $R$ constructed as in Lemma 55, that is, $f(x)=x \cdot\left(\begin{array}{c}c \\ b^{\prime}\end{array}\right), f(x)=t_{b b^{\prime}}\left(x,\left(\begin{array}{c}c \\ b^{\prime}\end{array}\right)\right)$, or $f(x)=h_{b b^{\prime}}\left(x,\left(\begin{array}{c}c \\ b\end{array}\right),\left(\begin{array}{c}c \\ b^{\prime}\end{array}\right)\right)$, depending on the type of $b b^{\prime}$. Then by Lemma55 $f: B \rightarrow B^{\prime}$ is a bijection, and therefore maps $\{c\} \times B \subseteq R$ onto $\{c\} \times B^{\prime}$, implying $\{c\} \times B^{\prime} \subseteq R$.

CASE 3. $c d$ and $b b^{\prime}$ are thin edges of the same type.

Let $B^{\prime}=b^{\prime \delta}$. Similar to Case 2 depending on the type of $c d$ we consider polynomial $f(x)=x \cdot\left(\begin{array}{c}d \\ b^{\prime}\end{array}\right), f(x)=t_{c d}\left(x,\left(\begin{array}{c}d \\ b^{\prime}\end{array}\right)\right)$, or $f(x)=h_{c d}\left(x,\left(\begin{array}{l}c \\ b\end{array}\right),\left(\begin{array}{c}d \\ b^{\prime}\end{array}\right)\right)$ for some $b \in B$. We have $f(c)=d$ and $f: B \rightarrow B^{\prime}$ is a bijection by Lemma[55, thus proving that $\{d\} \times B^{\prime} \subseteq R$.

If condition (1) holds the prove is essentially the same, except we need to use the same starting point as above, and consider pairs from $\operatorname{umax}(R \cap(F \times G))$. 
We are now in a position to state and prove the main result of the section. Let again $R$ be a subdirect product of $\mathbb{A}_{1} \times \mathbb{A}_{2}, \beta_{1}, \beta_{2}$ congruences of $\mathbb{A}_{1}, \mathbb{A}_{2}$ and $B_{1}, B_{2} \beta_{1}$ - and $\beta_{2}$-blocks, respectively. Also, let $R$ be chained with respect to $\left(\beta_{1}, \beta_{2}\right),\left(B_{1}, B_{2}\right)$ and $R^{*}=R \cap\left(B_{1} \times B_{2}\right), B_{1}^{*}=\operatorname{pr}_{1} R^{*}, B_{2}^{*}=\operatorname{pr}_{2} R^{*}$. Let $\alpha, \beta \in \operatorname{Con}\left(\mathbb{A}_{1}\right)$ be such that $\alpha \prec \beta \leq \beta_{1}$. This time we do not assume that $\operatorname{typ}(\alpha, \beta)=\mathbf{2}$.

Lemma 64 (The Congruence Lemma) Suppose $\alpha=\underline{0}_{1}$ and let $R^{\prime}$ be a polynomially closed subalgebra of $R^{*}$ and such that $B_{1}^{\prime}=\operatorname{pr}_{1} R^{\prime}$ contains an ascomponent $C$ of $B_{1}^{*}$ and $R^{\prime} \cap \operatorname{umax}\left(R^{*}\right) \neq \varnothing$. Then either

(1) $C \times \operatorname{umax}\left(B_{2}^{\prime \prime}\right) \subseteq R^{\prime}$, where $B_{2}^{\prime \prime}=R^{\prime}[C]$, or

(2) there is $\eta \in \operatorname{Con}\left(\mathbb{A}_{2}\right)$ with $\eta \prec \beta_{2}$ such that intervals $\left(\alpha, \beta_{1}\right)$ and $\left(\eta, \beta_{2}\right)$ cannot be separated.

Moreover, in case (2) $R^{\prime} \cap\left(C \times B_{2}^{\prime \prime}\right)$ is the graph of a mapping $\varphi: B_{2}^{\prime \prime} \rightarrow C$ such that the kernel of $\varphi$ is the restriction of $\eta$ on $B_{2}^{\prime \prime}$.

Proof: Note that if $|C|=1$, the lemma is trivially true. Let $B_{2}^{\prime}=\operatorname{pr}_{2} R^{\prime}$. We assume $\left.\beta_{2}\right|_{B_{2}^{\prime}} \neq\left.\lambda\right|_{B_{2}^{\prime}}$ for any congruence $\lambda \leq \beta_{2}$; otherwise replace $\beta_{2}$ with $\lambda$. Let $\mathrm{Ik}_{1}^{\prime}, \mathrm{Ik}_{2}^{\prime}$ be the link congruences of $B_{1}^{\prime}, B_{2}^{\prime}$ with respect to $R^{\prime}$. Let $\eta \leq \beta_{2}$ be

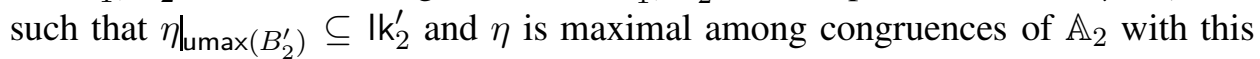
property. We show that either $\eta=\beta_{2}$ or it is one of the congruences in item (2) of the lemma. If $\eta$ is the full relation on umax $\left(B_{2}^{\prime}\right)$, we are done by Proposition 35, otherwise there are two cases.

CASE 1 . For some $\theta \in \operatorname{Con}\left(\mathbb{A}_{2}\right)$ with $\eta \prec \theta \leq \beta_{2}$ the intervals $\left(\underline{0}_{1}, \beta_{1}\right),(\eta, \theta)$ can be separated.

In this case we prove that $\eta$ has to be $\beta_{2}$ and we have option (1) of the lemma. Since $R$ is chained, by Lemmas $44(4)$ and 48 there is a set $T \subseteq B_{1}^{*} \times B_{1}^{*}$ of $\left(\underline{0}_{1}, \beta_{1}\right)$ subtraces such that any pair of elements from $\operatorname{umax}\left(B_{1}^{*}\right)$ belongs to the transitive closure of $T$, and for any $(a, b) \in T$ there is a $\left(B_{1}, B_{2}\right)$-preserving polynomial $f$ such that $f(a)=a, f(b)=b$, and $f\left(\left.\theta\right|_{B_{2}^{*}}\right) \subseteq \eta$. This means that $C$ belongs to the $\mathrm{Ik}_{1}^{*}$-block of $B_{1}^{*}$, where $\mathrm{Ik}_{1}^{*}$ is the link congruence with respect to $R^{*} / \eta$. Therefore $C \times \operatorname{umax}\left(R^{*}[C]\right) / \eta \subseteq R^{*} /{ }_{\eta}$. Observe that as $R^{\prime} \subseteq R^{*}$, the link congruence of $B_{1}^{*}$ with respect to $R^{*}$ restricted to $C$ contains $\left|\mathrm{k}_{1}^{\prime}\right|_{C}$. Therefore, we also have $C \times \operatorname{umax}\left(R^{*}[C]\right) \subseteq R^{*}$. Note that by the assumption $R^{\prime} \cap \operatorname{umax}\left(R^{*}\right) \neq \varnothing$ of the lemma both $R^{*}[C]$ and $B_{2}^{\prime}$ contain a u-maximal element from $B_{2}^{*}$. Since $B_{2}^{\prime \prime} \subseteq R^{*}[C]$, by Lemma 26 we have $\operatorname{umax}\left(B_{2}^{\prime \prime}\right) \subseteq \operatorname{umax}\left(R^{*}[C]\right)$. Therefore $C \times \operatorname{umax}\left(B_{2}^{\prime \prime}\right) \subseteq R^{*}$.

We are going to argue that the same inclusion holds for $R^{\prime}$. But first we show that for any thin semilattice or affine edge $a b$ of $C$ and any $c \in \operatorname{umax}\left(R^{*}[C]\right)$ 
there is a polynomial $g$ such that $g(a)=a, g(b)=b, f\left(\left.\theta\right|_{B_{2}^{*}}\right) \subseteq \eta$, and $g(c)=c$. Note that since $R$ is chained, all such pairs $\{a, b\}$ belong to $T$. Since every pair of elements of $C$ is a $\left(\underline{0}_{1}, \beta_{1}\right)$-subtrace, again, as $R$ is chained, and by Lemma 44(5) this is true for some $c \in R^{*}[C]$. Suppose $c c^{\prime}$ is a thin edge in $R^{*}[C]$; by Lemma 32 this implies that $(a, c),(b, c),\left(a, c^{\prime}\right),\left(b, c^{\prime}\right) \in R$. Then as in Lemma 57 we find a polynomial satisfying the required properties for $c^{\prime}$. Specifically, $g^{\prime}(x)=g(x)$. $\left(\begin{array}{l}a \\ c^{\prime}\end{array}\right), g^{\prime}(x)=t\left(g(x),\left(\begin{array}{l}a \\ c^{\prime}\end{array}\right)\right)$, and $g^{\prime}(x)=h^{\prime}\left(g(x),\left(\begin{array}{l}a \\ c\end{array}\right),\left(\begin{array}{l}a \\ c^{\prime}\end{array}\right)\right)$, where $t$ and $h$ are the operations from Lemma24(3), depending on the type of $c c^{\prime}$ and $a b$.

Now we are back to proving that $C \times \operatorname{umax}\left(B_{2}^{\prime \prime}\right) \subseteq R^{\prime}$. Observe that $R^{\prime} / \mathrm{Ik}_{1}^{\prime} \times \mathrm{Ik}_{2}^{\prime}$ is the graph of a bijective mapping $\varphi: B_{1}^{\prime} / \mathrm{Ik}_{1}^{\prime} \rightarrow B_{2}^{\prime} / \mathrm{Ik}_{2}^{\prime}$. Take $a, b \in C$ and $c \in \operatorname{umax}\left(B_{2}^{\prime \prime}\right)$ such that $(a, c) \in R^{\prime}, a b$ is a thin semilattice or affine edge and $(a, b) \notin \mathrm{Ik}_{1}^{\prime}$. Let also $(b, d) \in R^{\prime}$. By what is proved there is a polynomial $f$ of $R$ such that $f(a)=a, f(b)=b, f(c)=c \stackrel{\eta}{=} d^{\prime}=f(d)$, and $f\left(\left.\theta\right|_{B_{2}^{*}}\right) \subseteq$ $\eta$. In particular, $\left(a, d^{\prime}\right),\left(b, d^{\prime}\right) \in R$. Since $(a, c) \in R^{\prime}$ and $(a, c) \leq\left(b, c^{\prime}\right)$ or $(a, c)\left(b, c^{\prime}\right)$ is an affine edge for some $c^{\prime} \in \operatorname{Sg}\left(c, d^{\prime}\right)$, we obtain $\left(b, c^{\prime}\right) \in R^{\prime}$, as $R^{\prime}$ is polynomially closed. Since $c^{\prime} \stackrel{\eta}{\equiv} c$ and $\eta \leq \mathrm{Ik}_{2}^{\prime}$, we get a contradiction with $(a, b) \notin \mathrm{Ik}_{1}^{\prime}$.

CASE 2. For all $\theta \in \operatorname{Con}\left(\mathbb{A}_{2}\right)$ with $\eta \prec \theta \leq \beta_{2}$ the intervals $\left(\underline{0}_{1}, \beta_{1}\right),(\eta, \theta)$ cannot be separated.

Suppose $\left|k_{2}^{\prime}\right|_{B_{2}^{\prime \prime}} \nsubseteq \eta_{B_{2}^{\prime \prime}}$. Without loss of generality let $\eta=\underline{0}_{2}$. Then there are $a, b \in B_{2}^{\prime \prime}$ and $c \in C$ such that $(c, a),(c, b) \in R^{\prime}$. Let $\theta$ any congruence with $\eta \prec \theta \leq \operatorname{Cg}(\eta \cup\{(a, b)\}) \leq \beta_{2}$. If typ $\left(\underline{0}_{1}, \beta\right)=\mathbf{3}$ then by Lemma 49 such $a, b$ do not exists, as long as $\left(\underline{0}_{1}, \beta_{1}\right),(\eta, \theta)$ cannot be separated. Finally, if $\operatorname{typ}\left(\underline{0}_{1}, \beta\right) \in\{\mathbf{4}, \boldsymbol{5}\}, C$ is a singleton by Lemma 40 , and the result is trivial.

Suppose now that $\operatorname{typ}\left(\underline{0}_{1}, \beta_{1}\right)=\operatorname{typ}\left(\underline{0}_{2}, \theta\right)=\mathbf{2}$. In this case $B_{1}^{*}$ is a module, $C=B_{1}^{*}$ implying $B_{2}^{\prime}=B_{2}^{\prime \prime}$. Since $R$ is chained $a, b$ can be assumed to be from $\operatorname{umax}\left(B_{2}^{\prime \prime}\right)$, and so $\eta_{\text {umax }\left(B_{2}^{\prime \prime}\right)}<\left|k_{2}^{\prime}\right|_{\left(B_{2}^{\prime \prime}\right)}$. Also, this implies by Proposition 35 that for any $\mathrm{lk}_{1}^{\prime}$-block $E$ and the corresponding $\mathrm{lk}_{2}^{\prime}$-block $E^{\prime}$ it holds $E \times \operatorname{umax}\left(E^{\prime}\right) \subseteq$ $R^{\prime}$. Since by the choice of $\eta, \mathrm{Ik}_{2}^{\prime} \wedge \theta \not \leq \eta$, pairs $(c, a),(c, b)$ can be chosen such that $a \stackrel{\theta}{\equiv} b$. We prove that $\left.\theta\right|_{\text {umax }\left(B_{2}^{\prime \prime}\right)} \subseteq \mathrm{Ik}_{2}^{\prime}$ producing a contradiction with the choice of $\eta$.

By Lemma $58 R\left[B_{1}^{*}\right]$ is a subset of a $\zeta\left(\underline{0}_{2}, \theta\right)$-block. Then $\theta \leq \mathrm{Ik}_{2}^{\prime \prime} \vee \eta$, where $\mathrm{Ik}_{2}^{\prime \prime}$ is the link congruence of $\mathbb{A}_{2}$ with respect to $R$, and as $R$ is chained, by Lemma $\left.45 \theta\right|_{\text {umax }(E)} \leq\left|\mathrm{lk}_{2}^{*}\right|_{B_{2}^{*}}$ for any $\mathrm{lk}_{2}^{*}$-block $E \subseteq B_{2}^{*}$, since $B_{2}^{*} / \mathrm{lk}_{2}^{*}$ is a module, and therefore $E$ is u-maximal in this set. Thus we are in the conditions of Lemma 63. Therefore if $(c, d) \in R^{*}$ then $(c, e) \in R^{*}$ for any $e \stackrel{\theta}{=} d$ for any $c \in C$ and $d, e \in \operatorname{umax}(R[C])$. 
Again, we now extend this property to $R^{\prime}$ using the assumption that $R$ is polynomially closed. Since any pair $\left\{a^{\prime}, b^{\prime}\right\} \subseteq B_{2}^{*}$ with $a^{\prime} \stackrel{\theta}{\equiv} b^{\prime}$ is a $(\eta, \theta)$-subtrace, as $R$ is chained, there is a $\left(B_{1}, B_{2}\right)$-preserving polynomial $f$ such that $f(a)=a^{\prime}$ and $f(b)=b^{\prime}$. Now, use the pairs $(c, a),(c, b) \in R^{\prime}$. For any $b^{\prime} \in \operatorname{umax}\left(B_{2}^{\prime \prime}\right)$ with $b \stackrel{\theta}{\equiv} b^{\prime}$, let $b^{\prime \prime} \in b^{\theta}$ be such that $h\left(b, b^{\prime \prime}, a\right)=b^{\prime}$, where $h$ is the function from Theorem 20, such $b^{\prime \prime}$ exists because $h(b, x, a)$ is a permutation on every $\theta$-block (recall that a $\theta$-block is a module in this case). Since $R$ is chained, there is a polynomial $f$ such that $f(a)=a, f(b)=b^{\prime \prime}$ and $f(c)=d$ for some $d \in B_{1}^{*}=C$. The mapping $g(x)=h\left(x, f(x),\left(\begin{array}{l}d \\ a\end{array}\right)\right)$ is such that $g\left(\begin{array}{l}c \\ a\end{array}\right)=\left(\begin{array}{l}c \\ a\end{array}\right)$ and $g\left(\begin{array}{l}c \\ b\end{array}\right)=\left(\begin{array}{c}c \\ b^{\prime}\end{array}\right)$, because, again, $B_{1}^{*}$ is a module. Since $R^{\prime}$ is polynomially closed and $(c, b) \sqsubseteq a s\left(c, b^{\prime}\right)$ we have $\left(c, b^{\prime}\right) \in R^{\prime}$; and as $b^{\prime}$ is arbitrary from $a^{\theta}$, we have $\{c\} \times a^{\theta} \subseteq R^{\prime}$. Thus, we have proved the property for a specific $\theta$-block; next we extend it to other $\theta$ blocks.

Suppose $\{c\} \times E \subseteq R^{\prime}$ for some $\theta$-block $E$ and a $\theta$-block $E^{\prime}$ is such that for some $a \in E, b \in E^{\prime} \cap B_{2}^{\prime \prime}$, ab is a thin edge and $(d, b) \in R^{\prime}$ for some $d \in C$. Then by Lemma 55 mapping $g(x)$ that is defined as $x \cdot\left(\begin{array}{l}d \\ b\end{array}\right), t_{a b}\left(x,\left(\begin{array}{l}d \\ b\end{array}\right)\right)$, $h_{a b}\left(x,\left(\begin{array}{l}c \\ a\end{array}\right),\left(\begin{array}{l}d \\ b\end{array}\right)\right)$ depending on the type of $a b$ is injective on $E$. In particular, if $a b$ is semilattice or majority then $g$ maps $\{c\} \times E$ to $\{c\} \times E^{\prime}, g(c, a)=$ $(c, b), g\left(c, a^{\prime}\right)=\left(c, b^{\prime}\right)$ and $b \neq b^{\prime}$ whenever $a \neq a^{\prime}$; and since $t_{a b}, h_{a b}$ are term operations and all the tuples involved belong to $R^{\prime},(c, b),\left(c, b^{\prime}\right) \in R^{\prime}$. If $a b$ is affine then $g$ maps $\{c\} \times E$ to $\{d\} \times E^{\prime}$, and $g(c, a)=(d, b), g\left(c, a^{\prime}\right)=\left(d, b^{\prime}\right)$ and $b \neq b^{\prime}$ whenever $a \neq a^{\prime}$, and $(d, b),\left(d, b^{\prime}\right) \in R^{\prime}$. In either case, $\mathbf{I k}_{2}^{\prime}$ is nontrivial on $E^{\prime}$, and applying the argument from the previous paragraph we obtain $\{c\} \times E^{\prime} \subseteq R^{\prime}$ or $\{d\} \times E^{\prime} \subseteq R^{\prime}$. Therefore $\left.\theta_{\text {lumax }\left(B_{2}^{\prime}\right)} \subseteq \mathrm{Ik}_{2}^{\prime}\right|_{\mathrm{umax}\left(B_{2}^{\prime}\right)}$, a contradiction with the choice of $\eta$.

\section{Chaining}

In this section we first introduce a property of relations which is slightly stronger than chaining; this is the property that will be used in further proofs. Then we show in Lemma 65 that this property is preserved under certain transformations of the relation.

We call relation $R$ strongly chained with respect to $\bar{\beta}, \bar{B}$, where $\beta_{i} \in \operatorname{Con}\left(\mathbb{A}_{i}\right)$ and $B_{i}$ is a $\beta_{i}$-block for $i \in[n]$, if

(Q1s) for any $I \subseteq[n]$ and $\alpha, \beta \in \operatorname{Con}\left(\operatorname{pr}_{I} R\right)$ such that $\alpha \prec \beta \leq \bar{\beta}_{I}, \alpha$ and $\beta$ are 
$\mathcal{U}_{B}$-chained in $R$, where $\mathcal{U}_{B}$ is the set of all $\bar{B}$-preserving polynomials of $R$

(Q2s) for any $\alpha, \beta \in \operatorname{Con}\left(\operatorname{pr}_{I} R\right), \gamma, \delta \in \operatorname{Con}\left(\mathbb{A}_{j}\right), j \in[n]$, such that $\alpha \prec \beta \leq \bar{\beta}_{I}$, $\gamma \prec \delta \leq \beta_{j}$, and $(\alpha, \beta)$ can be separated from $(\gamma, \delta)$, the congruences $\alpha$ and $\beta$ are $\mathcal{U}^{*}$-chained in $R$, where $\mathcal{U}^{*}$ is the set of all $\bar{B}$-preserving polynomials $g$ of $R$ such that $g(\delta) \subseteq \gamma$

As in the definition of chained relations a polynomial from $\mathcal{U}^{*}$ in condition $(\mathrm{Q} 2 \mathrm{~s})$ will be called $(\gamma, \delta, \bar{B})$-good.

We now can state and prove Lemma 65 that the property to be strongly chained is preserved under certain transformations of $\bar{\beta}$ and $\bar{B}$. We will use it prove that one of the conditions, (S7), of a $\beta$-strategy (see Section 9.3) remains true when the $\beta$-strategy is being transformed.

Lemma 65 Let $R$ be a subdirect product of $\mathbb{A}_{1}, \ldots, \mathbb{A}_{n}, \beta_{i} \in \operatorname{Con}\left(\mathbb{A}_{i}\right)$ and $B_{i}$ a $\beta_{i}$-block, $i \in[n]$, such that $R$ is strongly chained with respect to $\bar{\beta}, \bar{B}$. Let $R^{\prime}=R \cap\left(B_{1} \times \cdots \times B_{n}\right)$ and $B_{i}^{\prime}=\operatorname{pr}_{i} R^{\prime}$. Fix $i \in[n], \beta_{i}^{\prime} \prec \beta_{i}$, and let $D_{i}$ be a $\beta_{i}^{\prime}$-block that is a member of a nontrivial as-component of $B_{i}^{\prime} / \beta_{\beta_{i}^{\prime}}$. Let also $\beta_{j}^{\prime}=\beta_{j}$ and $D_{j}=B_{j}$ for $j \neq i$. Then $R$ is strongly chained with respect to $\bar{\beta}^{\prime}, \bar{D}$.

Proof: Let $R^{\prime \prime}=R \cap\left(D_{1} \times \cdots \times D_{n}\right)$ and $D_{i}^{\prime}=\operatorname{pr}_{i} R^{\prime \prime}$. Take $I, j$ from the definition of being strongly chained. Let $I=[\ell]$; if $|I|>1$ we may consider $R$ as a subdirect product of $\operatorname{pr}_{I} R$ and $\mathbb{A}_{\ell+1}, \ldots, \mathbb{A}_{n}$, so we assume $|I|=1$ and $j=n$ in $(\mathrm{Q} 2 \mathrm{~s})$. Let $\alpha, \beta \in \operatorname{Con}\left(\mathbb{A}_{1}\right), \gamma, \delta \in \operatorname{Con}\left(\mathbb{A}_{n}\right)$ be such that $\alpha \prec \beta \leq \beta_{1}$, $\gamma \prec \delta \leq \beta_{n}$. Clearly, we may assume $\alpha=\underline{0}_{1}, \gamma=\underline{0}_{n}$, and $\beta_{i}^{\prime}=\underline{0}_{i}$. Note that replacing $R$ with the $n+1$-ary relation $\{(\mathbf{a}, \mathbf{a}[i]) \mid \mathbf{a} \in R\}$ we may assume that $i \notin I \cup\{j\}$. Without loss of generality assume $i=2$. By the assumption $\beta_{2}^{\prime}=\underline{0}_{2}$, the classes of $\beta_{2}^{\prime}$ are just elements of $\mathbb{A}_{2}$, so let $B_{2}^{\prime}$ be denoted by $c$. Let $C$ be the as-component of $B_{2}^{\prime}$ containing $c$.

To prove the lemma for every $a, b \in D_{1}^{\prime}$ with $a \stackrel{\beta}{\equiv} b$ we have to identify a set $T\left(a, b, \gamma, \delta, \mathcal{U}^{*}\right)$ as in conditions (G1),(G2), and for every $\left\{a^{\prime}, b^{\prime}\right\} \in T\left(a, b, \gamma, \delta, \mathcal{U}^{*}\right)$ we need to find a $(\gamma, \delta, \bar{D})$-good polynomial $f$ such that $f(a)=a^{\prime}, f(b)=$ $b^{\prime}$. In fact, we rather find all the sets $T$ minimal among the sets of the form $T\left(a, b, \gamma, \delta, \mathcal{U}^{*}\right)$ and that satisfy the conditions of Lemma 44(4). Note that such minimal sets exist for $\bar{\beta}, \bar{B}$, as well as, $(\gamma, \delta, \bar{B})$-good polynomials by the assumption that $R$ is strongly chained with respect to $\bar{\beta}, \bar{B}$. We need to change such a set $T$ and change the polynomials so that they fit the new requirements. We divide the proof into two cases, depending on whether or not $Q=\operatorname{pr}_{12} R^{\prime}$ is linked. First, we consider the case when $Q$ is not linked, this case is relatively easy.

CLAIM 1. Let $Q^{\prime}=Q \cap\left(\operatorname{umax}\left(\operatorname{pr}_{1} Q\right) \times C\right)$ be not linked and $\mathrm{Ik}_{1}, \mathrm{lk}_{2}$ link congruences of $Q$. Then $\mathrm{Ik}_{2}=\underline{0}_{2}$ and either $\beta \leq \mathrm{lk}_{1}$ or $\beta$ is trivial on $D_{1}$. 
Relation $Q$ is a subalgebra of $R \cap\left(B_{1} \times B_{2}\right)$ and is polynomially closed in $\operatorname{pr}_{12} R$ by Lemma 61. By the Congruence Lemma 64 if $Q^{\prime}$ is not linked then $Q$ is the graph of a mapping $\varphi: \operatorname{pr}_{1} Q \rightarrow C$. This means $\mathrm{Ik}_{2}=\underline{0}_{2}$ and $\mathrm{Ik}_{1}$ is the restriction of a congruence $\eta$ of $\mathbb{A}_{1}$ onto $\operatorname{pr}_{1} Q$. If $\beta \leq \eta$ then obtain the first option of the conclusion of the claim, otherwise $\mathrm{Ik}_{1} \cap \beta=\underline{0}_{1}$ and we have the second option.

Note that if $\beta \leq \mathrm{l}_{1}$ then any $\bar{B}$-preserving polynomial that maps a pair of $\beta$-related elements from $D_{1}^{\prime}$ on a $(\alpha, \beta)$-subtrace from $D_{1}^{\prime}$ is also $\bar{D}$-preserving, because $\mathrm{Ik}_{2}=\underline{0}_{2}$; the result follows. If $\beta$ is trivial on $D_{1}^{\prime}$, there is nothing to prove. Therefore we may assume $Q^{\prime}$ is linked.

We start with choosing a $\beta$-block required in the chaining conditions, and studying some of its properties. Observe that since $c$ is as-maximal in $B_{2}^{\prime}$, the set $D_{1}^{\prime}$ also contains as-maximal elements of $B_{1}^{\prime}$. Therefore by Lemma26 umax $\left(D_{1}^{\prime}\right) \subseteq$ $\operatorname{umax}\left(B_{1}^{\prime}\right)$. Let $E$ be a $\beta$-block such that $E^{\prime \prime}=E \cap D_{1}^{\prime} \neq \varnothing, E \cap \operatorname{umax}\left(D_{1}^{\prime}\right) \neq \varnothing$ (and so $E^{\prime \prime}$ satisfies the requirements of the chaining conditions), and let $E^{\prime}=$ $E \cap B_{1}^{\prime}$. Consider $R^{*}=R^{\prime} \cap\left(B_{1} \times C \times B_{3} \times \cdots \times B_{n}\right)$. Note that $R^{*}$ is not necessarily a subalgebra. Let $B_{i}^{*}=\operatorname{pr}_{i} R^{*}, i \in[n]$, and $E^{*}=E \cap B_{1}^{*}$. By the Maximality Lemma 27(4) amax $\left(E^{*}\right)$ is a union of as-components of $E^{\prime}$. Indeed, let $a \in E^{*}$ and let $\mathbf{a} \in R^{*}$ be such that $\mathbf{a}[1]=a$ and $\mathbf{a}[2] \in C$; let also $b \in E^{\prime}$ with $a \sqsubseteq a s b$ in $E^{\prime}$. Then by the Maximality Lemma 27(4) there is $\mathbf{b} \in R^{\prime}$ such that $\mathbf{b}[1]=b$ and $\mathbf{a} \sqsubseteq_{a s} \mathbf{b}$ in $R^{\prime}$. In particular, $\mathbf{a}[2] \sqsubseteq_{a s} \mathbf{b}[2]$ implying $\mathbf{b}[2] \in C$. Also, by Proposition 35, since $Q$ is linked and umax $\left(E^{*}\right) \subseteq \operatorname{umax}\left(B_{1}^{\prime}\right)$, we have $\operatorname{umax}\left(E^{*}\right) \times C \subseteq Q$, and therefore $\operatorname{umax}\left(E^{*}\right)=\operatorname{umax}\left(E^{\prime \prime}\right) \subseteq \operatorname{umax}\left(E^{\prime}\right)$. In particular, $\operatorname{amax}\left(E^{\prime \prime}\right)$ is a union of as-components of $E^{\prime}$. The last inclusion here is because $E^{*}$ contains some as-maximal elements of $E^{\prime}$.

First we prove condition (Q1s) for $\bar{\beta}^{\prime}$ and $\bar{D}$.

Claim 2. For any $a, b, a^{\prime}, b^{\prime} \in E^{\prime \prime}$ such that $a, b$ belong to the same ascomponent of $E^{\prime \prime}$ there is a $(\gamma, \delta, \bar{D})$-good polynomial $f$ with $f\left(\left\{a^{\prime}, b^{\prime}\right\}\right)=\{a, b\}$.

Consider relation $S$, a subdirect product of $\mathbb{A}_{1} \times \mathbb{A}_{1} \times \mathbb{A}_{2} \times \cdots \times \mathbb{A}_{n}$, produced from by $\left(a^{\prime}, b^{\prime}, \mathbf{a}\right)$, where $\mathbf{a} \in \operatorname{pr}_{\{2, \ldots, n\}} R^{\prime \prime}$, as follows:

$$
S=\{f(a), f(b), f(\mathbf{a})) \mid f \text { is a unary polynomial of } R \text { with } f(\delta) \subseteq \gamma\} .
$$

It is not difficult to see that $S$ is a subalgebra, and, in particular it contains all the tuples of the form $(\mathbf{b}[1], \mathbf{b}[1], \mathbf{b}[2], \ldots, \mathbf{b}[n])$ for $\mathbf{b} \in R$. Let $S^{\prime}=S \cap \bar{B}$, and $S^{\prime \prime}=S \cap \bar{D}$. Every tuple from $S^{\prime}$ or from $S^{\prime \prime}$ corresponds to a $\bar{B}$ - or $\bar{D}$-preserving polynomial. Therefore it suffices to prove that $(a, b) \in \operatorname{pr}_{12} S^{\prime \prime}$. Let $F$ be the ascomponent of $E^{\prime \prime}$ containing $a, b$; as observed above $F$ is also an as-component of $E^{\prime}$. By the assumption of (Q2s) $F^{2} \subseteq \operatorname{pr}_{12} S^{\prime}$ and $(e, e) \in \operatorname{pr}_{12} S^{\prime \prime}$ for any $e \in F$, 
since $F \times C \subseteq Q^{\prime}$. We consider relation $P=\operatorname{pr}_{123} S^{\prime}$. As $F^{2} \subseteq P^{\prime}=\operatorname{pr}_{12} P$, $(a, b)$ is as-maximal in $P^{\prime}$. Therefore it suffices to show that $\operatorname{amax}(P)$ is linked when considered as subdirect product of $P^{\prime}$ and $B_{2}^{\prime}$. Since $(e, e) \in \operatorname{pr}_{12} S^{\prime \prime}$ for any $e \in F$, all pairs of this form are linked in $P$. Then $\left(e, d, a^{\prime \prime}\right) \in P$ for any $e, d \in F$ and some $a^{\prime \prime} \in B_{2}^{\prime}$, and $\left(e, e, c^{\prime \prime}\right) \in P$ for some $c^{\prime \prime} \in C$. Since $F^{2} \subseteq P^{\prime}$, $(e, e) \sqsubseteq a s(e, d)$, and by the Maximality Lemma 27(4) $a^{\prime \prime}$ can be chosen from $C$, and so this implies that $(e, d)$ and $(e, e)$ are also linked. Claim 2 is proved.

Now we extend the result above to pairs from $\operatorname{umax}\left(E^{*}\right)$. We prove the result in two steps. First, we show that for any $a^{\prime}, b^{\prime} \in E^{*}$ and any $a, b \in \operatorname{umax}\left(E^{*}\right)$ there is a sequence of $\bar{B}$-preserving polynomials $f_{1}, \ldots, f_{k}$ such that $f_{1}\left(\left\{a^{\prime}, b^{\prime}\right\}\right), \ldots, f_{k}\left(\left\{a^{\prime}, b^{\prime}\right\}\right) \subseteq$ $E^{*}$ form a chain connecting $a$ and $b, f_{i}\left(\mathbb{A}_{1}\right)$ is an $(\alpha, \beta)$-minimal set, and $f_{i}(c) \in C$ for $i \in[k]$. Then we prove that $f_{1}, \ldots, f_{k}$ can be chosen in such a way that $f_{1}\left(\left\{a^{\prime}, b^{\prime}\right\}\right), \ldots, f_{k}\left(\left\{a^{\prime}, b^{\prime}\right\}\right) \subseteq E^{\prime \prime}$ and $f_{1}(c)=\cdots=f_{k}(c)=c$. Clearly, it suffices to prove in the case when $b$ is as-maximal in $E^{*}$.

By the assumption there are $a=a_{1}, a_{2}, \ldots, a_{k}=b, a_{1}, \ldots, a_{k} \in E^{\prime}$ and $(\gamma, \delta, \bar{B})$-good polynomials $f_{1}, \ldots, f_{k-1}$ such that $f_{i}\left(\mathbb{A}_{1}\right)$ is a $(\alpha, \beta)$-minimal set and $f_{i}\left(\left\{a^{\prime}, b^{\prime}\right\}\right)=\left\{a_{i}, a_{i+1}\right\}$, and also $f_{i}(c) \in B_{2}^{\prime}$. We need to show that $a_{1}, \ldots, a_{k-1}$ and $f_{1}, \ldots, f_{k-1}$ can be chosen such that $f_{i}(c) \in C$. Choose $\mathbf{a}, \mathbf{b} \in R^{\prime \prime}$ such that $\mathbf{a}[1]=a, \mathbf{b}[1]=b$ and $\mathbf{a}[2]=\mathbf{b}[2]=c$. This is possible because umax $\left(E^{*}\right)=$ $\operatorname{umax}\left(E^{\prime \prime}\right)$. Now let $g_{i}(x)=\operatorname{maj}\left(\mathbf{a}, f_{i}(x), \mathbf{a}\right)$ and $h_{i}(x)=\operatorname{maj}\left(\mathbf{a}, \mathbf{b}, f_{i}(x)\right)$. By Lemma $44 g_{i}, h_{i}$ are $(\gamma, \delta, \bar{B})$-good polynomials, and for each of them either $\left\{b_{i}, b_{i+1}\right\}=g_{i}\left(\left\{a^{\prime}, b^{\prime}\right\}\right)\left(\left\{c_{i}, c_{i+1}\right\}=h_{i}\left(\left\{a^{\prime}, b^{\prime}\right\}\right)\right)$ is an $(\alpha, \beta)$-subtrace, or $g_{i}(\beta) \subseteq \alpha\left(h_{i}(\beta) \subseteq \alpha\right)$, that is $g_{i}\left(a^{\prime}\right)=g_{i}\left(b^{\prime}\right)$ (respectively, $h_{i}\left(a^{\prime}\right)=h_{i}\left(b^{\prime}\right)$ ). The polynomials $g_{i}, h_{i}$ satisfying the first option form a sequence of $(\alpha, \beta)$-subtraces connecting $a$ with $\operatorname{maj}(a, b, a)$ - by subtraces of the form $\left\{b_{i}, b_{i+1}\right\}$, - and $\operatorname{maj}(a, b, a)$ with $\operatorname{maj}(a, b, b)$ - by subtraces of the form $\left\{c_{i}, c_{i+1}\right\}$. Also, by Theorem $31 \operatorname{maj}(a, b, b)$ belongs to the as-component of $E^{*}$ (and therefore of $E^{\prime}$ and $\left.E^{\prime \prime}\right)$ containing $b$. Therefore by Claim 2 this sequence of polynomials and subtraces can be continued to connect $\operatorname{maj}(a, b, b)$ to $b$. Finally, by the same theorem $g_{i}(c)=\operatorname{maj}\left(c, f_{i}(c), c\right) \in C$ and $h_{i}(c)=\operatorname{maj}\left(c, c, f_{i}(c)\right) \in C$.

For the second step we assume that $a$ and $b$ are connected with $(\alpha, \beta)$-subtraces $\left\{a_{i}, a_{i+1}\right\}, i \in[k-1]$ witnessed by $(\gamma, \delta, \bar{B})$-good polynomials $f_{i}$ such that $c_{i}=f_{i}(c) \in C$. We need to show that $f_{i}$ can be chosen such that $f_{i}(c)=c$. Suppose that $c_{i} \neq c$ for some $i \in[k-1]$. Since $c_{i}$ and $c$ belong to the same as-component, there is an as-path $c_{i}=d_{1}, \ldots, d_{\ell}=c$ in $C$. We show that if there is a sequence of $(\alpha, \beta)$-subtraces $\left\{b_{j}, b_{j+1}\right\}$ witnessed by polynomials $g_{j}$ such that $g_{j}(c)=c$ whenever $f_{j}(c)=c$, and $f_{i}(c)=d_{t}$, there are also $(\alpha, \beta)$ subtraces $\left\{b_{j}^{\prime}, b_{j+1}^{\prime}\right\}$ such that $b_{1}^{\prime}=a$ and $b_{k}^{\prime}$ is in the as-component containing $b$, witnessed by polynomials $g_{1}^{\prime}, \ldots, g_{k}^{\prime}$ such that $g_{i}^{\prime}(c)=d_{t+1}$ and $g_{j}^{\prime}(c)=c$ 
whenever $g_{j}(c)=c$.

As is easily seen, it suffices to find a ternary term operation $p$ such that $p(a, a, b)$ belongs to the as-component containing $b$, and $p\left(d_{t+1}, d_{t}, d_{t}\right)=d_{t+1}$. Indeed, if such a term operation exists, then we set $g_{j}^{\prime}(x)=p\left(\mathbf{a}, \mathbf{a}, g_{j}(x)\right)$, where $\mathbf{a}$ is as in the first step above, for $j \in[k-1]-\{i\}$, and $\left\{b_{j}^{\prime}, b_{j+1}^{\prime}\right\}=g_{j}^{\prime}\left(\left\{a^{\prime}, b^{\prime}\right\}\right)$. We have $g_{1}^{\prime}\left(a^{\prime}\right)=p\left(a, a, g_{1}\left(a^{\prime}\right)\right)=a$ and $g_{j}^{\prime}(c)=p\left(c, c, g_{j}(c)\right)=c$ whenever $g_{j}(c)=c$. Finally, since $g_{\ell}^{\prime}(b)=p(a, a, b)$ belongs to the as-component containing $b$, we can use Claim 2 as before to connect $p(a, a, b)$ to $b$. For $g_{i}^{\prime}$ we set $g_{i}^{\prime}(x)=$ $p\left(\mathbf{a}^{\prime}, \mathbf{a}^{\prime \prime}, g_{i}(x)\right)$ where $\mathbf{a}^{\prime}, \mathbf{a}^{\prime \prime} \in R^{\prime \prime}$ are such that $\mathbf{a}^{\prime}[1]=\mathbf{a}^{\prime \prime}[1]=a$ and $\mathbf{a}^{\prime}[2]=$ $d_{t+1}, \mathbf{a}^{\prime \prime}[2]=d_{t}$. Note that such $\mathbf{a}^{\prime}, \mathbf{a}^{\prime \prime}$ exist, because $\operatorname{umax}\left(E^{*}\right) \times C \subseteq Q$. It follows from the assumption about $p$ that $g_{i}^{\prime}$ is as required.

If $d_{t} \leq d_{t+1}$, then $p(x, y, z)=z \cdot x$ fits the requirements. If $d_{t} d_{t+1}$ is an affine edge, consider the relation $S \subseteq \mathbb{A}_{1} \times \mathbb{A}_{2}$ generated by $\left\{\left(a, d_{t}\right),\left(a, d_{t+1}\right),\left(b, d_{t}\right)\right\}$. Let $\mathbb{B}=\operatorname{Sg}(a, b)$ and $\mathbb{C}=\operatorname{Sg}\left(d_{t}, d_{t+1}\right)$; then $\mathbb{B} \times\left\{d_{t}\right\},\{a\} \times \mathbb{C} \subseteq S$. By Lemma 32, as $d_{t} d_{t+1}$ is a thin affine edge, $\operatorname{umax}(\mathbb{B}) \times\left\{d_{t+1}\right\} \subseteq S$. There is $b^{\prime}$ with $b \sqsubseteq_{a s} b^{\prime}$ in $\mathbb{B}$ such that $b^{\prime} \in \operatorname{umax}(\mathbb{B})$. Therefore there is a term operation $p$ with $p(a, a, b)=b^{\prime}$ and $p\left(d_{t+1}, d_{t}, d_{t}\right)=d_{t+1}$, as required.

\section{Strategies and solutions}

\subsection{The grand scheme}

In this section we describe the 'grand scheme' of solving CSPs. We start with introducing two preprocessing steps for our algorithm.

We call a CSP instance $\mathcal{P}=(V, \mathcal{C})$ subdirectly irreducible if it is 1-minimal and $\mathbb{A}_{v}$ is subdirectly irreducible for every $v \in V$.

Lemma 66 (Folklore) Every CSP instance can be reduced in polynomial time to an equivalent subdirectly irreducible one.

In this section all instances we consider are assumed subdirectly irreducible. The monolith of $\mathbb{A}_{v}$ is denoted by $\mu_{v}$.

Let $\mathcal{P}=(V, \mathcal{C})$ be a $(2,3)$-minimal instance and for $X \subseteq V,|X|=2$, there is a constraint $C^{X}=\left\langle X, R^{X}\right\rangle$, where $R^{X}$ is the set of partial solutions on $X$. We use the notation from the end of Section 6.2. Recall that $\mathcal{W}^{\mathcal{P}}(\bar{\beta})$ denotes the set of triples $(v, \alpha, \beta)$ such that $v \in V, \alpha, \beta \in \operatorname{Con}\left(\mathbb{A}_{v}\right)$, and $\alpha \prec \beta \leq \beta_{v}$. If $\beta_{v}=\underline{1}_{v}$ for all $v \in V$, we set $\mathcal{W}^{\mathcal{P}} \mathcal{W}^{\mathcal{P}}(\bar{\beta})$. Also, let $W_{v, \alpha \beta, \bar{\beta}}$ to be the set of $w \in V$ such that for some $(w, \gamma, \delta) \in \mathcal{W}^{\mathcal{P}}(\bar{\beta})$ the prime intervals $(\alpha, \beta)$ and $(\gamma, \delta)$ cannot be separated in $R^{\{v, w\}}$. Let $\bar{\beta}, \beta_{v} \in \operatorname{Con}\left(\mathbb{A}_{v}\right), v \in V$, be a collection of 
congruences. Let $\mathcal{W}^{\prime}(\bar{\beta})$ (and respectively $\mathcal{W}^{\prime}$ ) denote the set of triples $(v, \alpha, \beta) \in$ $\mathcal{W}(\bar{\beta})$ (respectively, from $\mathcal{W}$ ) with $\zeta(\alpha, \beta)=\underline{1}_{v}$.

We say that algebra $\mathbb{A}_{v}$ is semilattice free if it does not contain semilattice edges. Let $\operatorname{size}(\mathcal{P})$ denote the maximal size of domains of $\mathcal{P}$ that are not semilattice free and $\operatorname{MAX}(\mathcal{P})$ be the set of variables $v \in V$ such that $\left|\mathbb{A}_{v}\right|=\operatorname{size}(\mathcal{P})$ and $\mathbb{A}_{v}$ is not semilattice free. Finally, for $Y \subseteq V$ let $\mu_{v}^{Y}=\mu_{v}$ if $v \in Y$ and $\mu_{v}^{Y}=\underline{0}_{v}$ otherwise. Recall that by $\mathcal{P} / \bar{\mu}^{Y}$ we denote the instance $\left(V, \mathcal{C}^{\bar{\mu}^{Y}}\right)$ constructed as follows: the domain of $v \in V$ is $\mathbb{A}_{v} / \mu_{v}^{Y}$; for every constraint $C=\langle\mathbf{s}, R\rangle \in \mathcal{C}$, the set $\mathcal{C}^{\bar{\mu}^{Y}}$ includes the constraint $\left\langle\mathbf{s}, R / \bar{\mu}_{\mathbf{s}}^{Y}\right\rangle$.

Instance $\mathcal{P}$ is said to be block-minimal if for every $(v, \alpha, \beta) \in \mathcal{W}$ (here $\beta_{v}=$ $\left.\underline{1}_{v}, v \in V\right)$

(BM1) for every $C=\langle\mathbf{s}, R\rangle \in \mathcal{C}$ the problem $\mathcal{P}_{W_{v, \alpha \beta},}$ if $(v, \alpha, \beta) \notin \mathcal{W}^{\prime}$, and the problem $\mathcal{P}_{W_{v, \alpha \beta, \bar{\beta}}} / \bar{\mu}^{Y}$ otherwise, where $Y=\operatorname{MAX}(\mathcal{P})-\mathbf{s}$, is minimal;

(BM2) if $(v, \alpha, \beta) \in \mathcal{W}^{\prime}$, then for every $(w, \gamma, \delta) \in \mathcal{W}-\mathcal{W}^{\prime}$ the problem $\mathcal{P}_{W_{v, \alpha \beta, \bar{\beta}}} / \bar{\mu}^{Y}$, where $Y=\operatorname{MAX}(\mathcal{P})-\left(W_{v, \alpha \beta, \bar{\beta}} \cap W_{w, \gamma \delta, \bar{\beta}}\right)$ is minimal.

The definition of block-minimality is designed in such a way that block-minimality can be efficiently established. Observe that $W_{v, \alpha \beta, \bar{\beta}}$ can be large, even equal to $V$. However if $(v, \alpha, \beta) \notin \mathcal{W}^{\prime}$ by Theorem 59 the problem $\mathcal{P}_{W_{v, \alpha \beta}, \bar{\beta}}$ splits into a union of disjoint problems over smaller domains. On the other hand, if $(v, \alpha, \beta) \in \mathcal{W}^{\prime}$ then $\mathcal{P}_{W_{v, \alpha \beta, \bar{\beta}}}$ may not be decomposable. Since we need an efficient procedure of establishing block-minimality, this explains the complications introduced in (BM1),(BM2).

For an instance $\mathcal{P}$ we say that an instance $\mathcal{P}^{\prime}$ is strictly smaller than instance $\mathcal{P}$ if $\operatorname{size}\left(\mathcal{P}^{\prime}\right)<\operatorname{size}(\mathcal{P})$.

Lemma 67 Let $\mathcal{P}=(V, \mathcal{C})$ be a (2,3)-minimal instance. Then $\mathcal{P}$ can be transformed to an equivalent block-minimal instance $\mathcal{P}^{\prime}$ by solving a quadratic number of strictly smaller CSPs.

Proof: To establish block-minimality of $\mathcal{P}$, for every $(v, \alpha, \beta) \in \mathcal{W}$ (let $\left.W=W_{v, \alpha \beta}\right)$, we need to check if the problems given in conditions (BM1),(BM2) are minimal. If they are then $\mathcal{P}$ is block-minimal, otherwise some tuples can be removed from some constraint relation $R$ (the set of tuples that remain in $R$ is always a subalgebra, as is easily seen), and the instance $\mathcal{P}$ tightened, in which case we need to repeat the procedure with the tightened instance. Therefore we just need to show how to reduce solving those subproblems to solving strictly smaller CSPs. 
For $C=\langle\mathbf{s}, R\rangle \in \mathcal{C}$ and $\mathbf{a} \in R$ let $\mathcal{P}^{\prime}$ be the problem obtained as follows: fix the values of variables from $\mathbf{s} \cap W$, or from $\mathbf{s} \cap W \cap W_{w, \gamma \delta}$ in the case of (BM2) to those of a. If the resulting problem is $\mathcal{P}^{\prime \prime}$ then set $\mathcal{P}^{\prime}=\mathcal{P}^{\prime \prime} / \bar{\mu}^{Y}$, where $Y$ is either empty, if $(v, \alpha, \beta) \notin \mathcal{W}^{\prime}$, or $Y=\operatorname{MAX}(\mathcal{P})-\mathbf{s}$, if $(v, \alpha, \beta) \in \mathcal{W}^{\prime}$ in (BM1), or $Y=\operatorname{MAX}(\mathcal{P})-\left(W \cap M_{w, \gamma \delta}\right)$ in (BM2). In the first case, by Theorem 59 $\mathcal{P}^{\prime}$ is a disjoint union of instances $\mathcal{P}_{1}, \ldots, \mathcal{P}_{\ell}$ and $\operatorname{size}\left(\mathcal{P}_{i}\right)<\operatorname{size}(\mathcal{P})$. In the second case the domains of variables from $\mathbf{s} \cap W$ have cardinality 1 , and the domain of each of the remaining variables either is semilattice free, or is smaller than $\operatorname{size}(\mathcal{P})$. Finally, in the last case the domain of each of the variables outside of $W \cap W_{w, \gamma \delta}$ is either semilattice free or smaller than $\operatorname{size}(\mathcal{P})$. Also, by Theorem $59 \mathcal{P}_{W \cap W_{w, \gamma \delta}}$ is a disjoint union of instances with domains of smaller size. Let $\mathcal{P}_{1}, \ldots, \mathcal{P}_{k}$ be these disjoint instances. Then $\mathcal{P}^{\prime}$ can be reduced to solving the instances $\mathcal{P}_{1}^{\prime}, \ldots, \mathcal{P}_{k}^{\prime}$ obtained from $\mathcal{P}^{\prime}$ by restricting $\mathcal{P}_{W \cap W_{w, \gamma \delta}}^{\prime}$ to $\mathcal{P}_{i}$. This completes the proof.

Let $\mathcal{P}=(V, \mathcal{C})$ be a subdirectly irreducible $(2,3)$-minimal instance. Let $\operatorname{Center}(\mathcal{P})$ denote the set of variables $v \in V$ such that $\zeta\left(\underline{0}_{v}, \mu_{v}\right)=\underline{1}_{v}$. Let $\mu_{v}^{*}=\mu_{v}$ if $v \in \operatorname{MAX}(\mathcal{P}) \cap \operatorname{Center}(\mathcal{P})$ and $\mu_{v}^{*}=\underline{0}_{v}$ otherwise.

We consider several cases and indicate what kind of reductions or solution algorithms we intend to use in each case.

Case 1: Semilattice free domains. If all domains of $\mathcal{P}$ are semilattice free then $\mathcal{P}$ can be solved in polynomial time, using the few subpowers algorithm, as shown in [21].

Case 2: Collapsing trivial centralizers. If $\mu_{v}^{*}=\underline{0}_{v}$ for all $v \in V$, blockminimality guarantees that a solution exists, and we can use Lemma 67 to solve the instance.

Theorem 68 If $\mathcal{P}$ is subdirectly irreducible, (2,3)-minimal, block-minimal, and $\operatorname{MAX}(\mathcal{P}) \cap \operatorname{Center}(\mathcal{P})=\varnothing$, then $\mathcal{P}$ has a solution.

Case 3: Nontrivial centralizers. If $\operatorname{MAX}(\mathcal{P}) \cap \operatorname{Center}(\mathcal{P})=\varnothing$, we first solve the problem $\mathcal{P} / \mu^{*}$, and then use Theorem 69 to reduce $\mathcal{P}$ to a strictly smaller instance. An efficient way to establish 1-minimality of $\mathcal{P} / \mu^{*}$ is given in Theorem 70 ,

Theorem 69 If $\mathcal{P} / \bar{\mu}^{*}$ is 1-minimal, then $\mathcal{P}$ can be reduced in polynomial time to a strictly smaller instance.

With the reductions above a solution algorithm goes as shown in Algorithm 2 , we reproduce it here for convenience. 


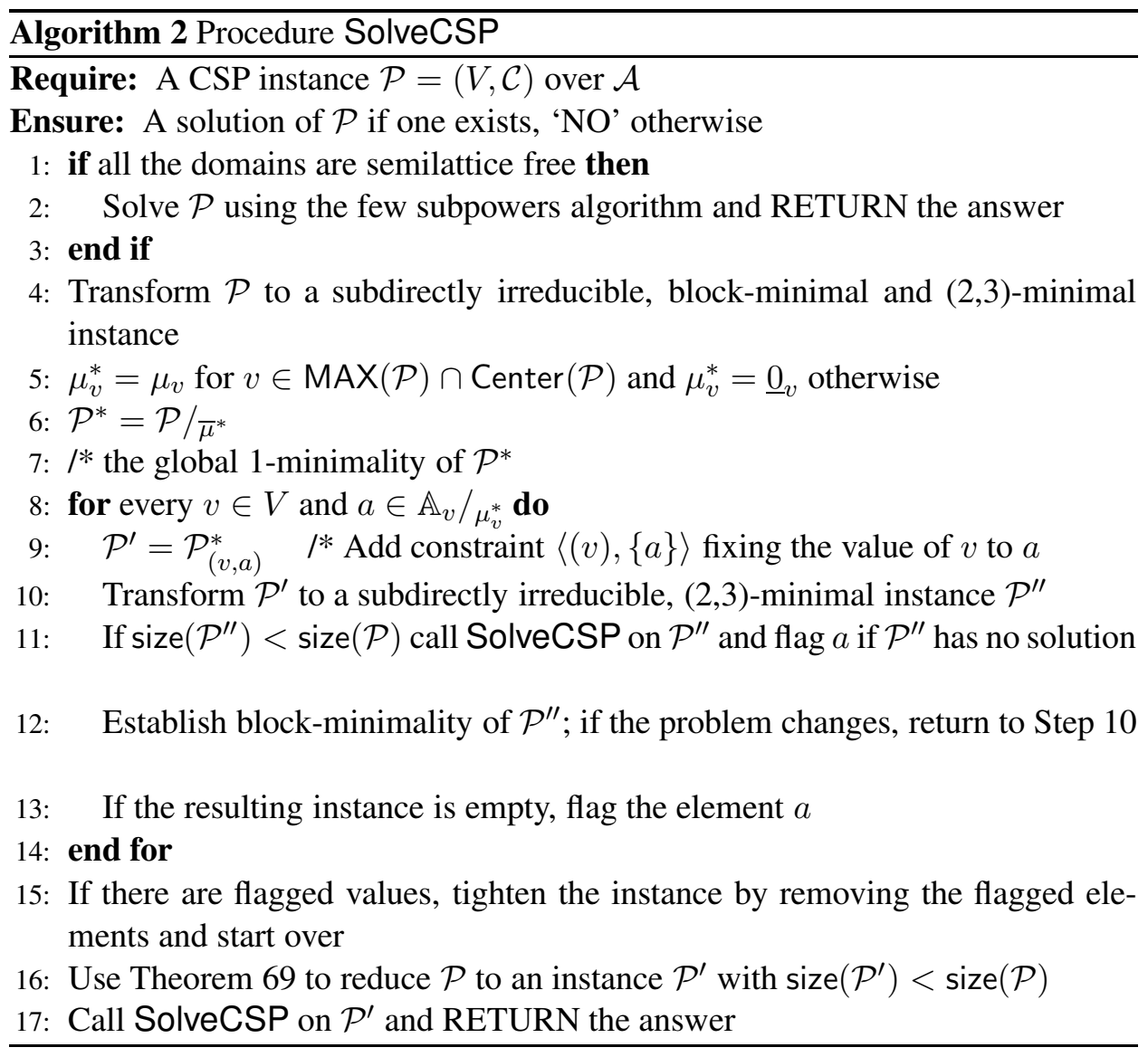


Theorem 70 Algorithm SolveCSP (Algorithm 2) correctly solves every instance from $\operatorname{CSP}(\mathcal{A})$ and runs in polynomial time.

Proof: By the results of [21] the algorithm correctly solves the given instance $\mathcal{P}$ in polynomial time if the conditions of Step 1 are true. Lemma67implies that Steps 4 and 12 can be completed by recursing to strictly smaller instances.

Next we show that the for-loop in Steps 8-14 checks if $\mathcal{P}^{*}=\mathcal{P} / \bar{\mu}^{*}$ is globally 1-minimal. For this we need to verify that a value $a$ is flagged if and only if $\mathcal{P}^{*}$ has no solution $\varphi$ with $\varphi(v)=a$, and therefore if no values are flagged then $\mathcal{P}^{*}$ is globally 1-minimal. If $\varphi(v)=a$ for some solution $\varphi$ of $\mathcal{P}^{*}$, then $\varphi$ is a solution $\mathcal{P}^{\prime}$ constructed in Step 9. In this case Steps 11,12 cannot result in an empty instance. Suppose $a \in \mathbb{A}_{v} / \mu_{v}^{*}$ is not flagged. If $\operatorname{size}\left(\mathcal{P}^{\prime \prime}\right)<\operatorname{size}(\mathcal{P})$ this means that $\mathcal{P}^{\prime \prime}$ and therefore $\mathcal{P}^{\prime}$ has a solution. Otherwise this means that establishing blockminimality of $\mathcal{P}^{\prime \prime}$ is successful. In this case $\mathcal{P}^{\prime \prime}$ has a solution by Theorem 68, because $\operatorname{MAX}\left(\mathcal{P}^{\prime \prime}\right) \cap \operatorname{Center}\left(\mathcal{P}^{\prime \prime}\right)=\varnothing$. This in turn implies that $\mathcal{P}^{\prime}$ has a solution. Observe also that the set of unflagged values for each variable $v \in V$ is a subalgebra of $\mathbb{A} / \mu^{*}$. Indeed, the set of solutions of $\mathcal{P}^{*}$ is a subalgebra $\mathcal{S}^{*}$ of $\prod_{v \in V} \mathbb{A} / \mu^{*}$, and the set of unflagged values is the projection of $\mathcal{S}^{*}$ of the coordinate position $v$.

Finally, if Steps 8-15 are completed without restarts, Steps 16,17 can be completed by Theorem 69 and recursing on $\mathcal{P}^{\prime}$ with $\operatorname{size}\left(\mathcal{P}^{\prime}\right)<\operatorname{size}(\mathcal{P})$.

To see that the algorithm runs in polynomial time it suffices to observe that

(1) The number of restarts in Steps 4 and 15 is at most linear, as the instance becomes smaller after every restart; therefore the number of times Steps 4-15 are executed together is at most linear.

(2) The number of iterations of the for-loop in Steps 8-14 is linear.

(3) The number of restarts in Steps 10 and 12 is at most linear, as the instance becomes smaller after every iteration.

(4) Every call of SolveCSP when establishing block-minimality in Steps 4, and 12 is made on an instance strictly smaller than $\mathcal{P}$, and therefore depth of recursion is bounded by $\operatorname{size}(\mathcal{P})$ in Step 4,11,12 and 17 .

Thus a more thorough estimation gives a bound on the running time of $O\left(n^{3 k}\right)$, where $k$ is the maximal size of an algebra in $\mathcal{A}$.

\subsection{Proof of Theorem 69}

Following [53] let $\mathcal{P}=(V, \mathcal{C})$ be an instance and $p_{v}: \mathbb{A}_{v} \rightarrow \mathbb{A}_{v}, v \in V$. Mappings $p_{v}, v \in V$, are said to be consistent if for any $\langle\mathbf{s}, R\rangle \in \mathcal{C}, \mathbf{s}=\left(v_{1}, \ldots, v_{k}\right)$, and any tuple $\mathbf{a} \in R$ the tuple $\left(p_{v_{1}}(\mathbf{a}[1]), \ldots, p_{v_{k}}(\mathbf{a}[k])\right)$ belongs to $R$. It is easy to see that the composition of two families of consistent mappings is also a consistent 
mapping. For consistent idempotent mappings $p_{v}$ by $p(\mathcal{P})$ we denote the retraction of $\mathcal{P}$, that is, $\mathcal{P}$ restricted to the images of $p_{v}$. In this case $\mathcal{P}$ has a solution if and only if $p(\mathcal{P})$ has, see [53].

Let $\varphi$ be a solution of $\mathcal{P} / \bar{\mu}^{*}$. We define $p_{v}^{\varphi}: \mathbb{A}_{v} \rightarrow \mathbb{A}_{v}$ as follows: $p_{v}^{\varphi}=q_{v}^{k}$, where $q_{v}(a)=a \cdot b_{v}$, element $b_{v}$ is any element of $\varphi(v)$, and $k$ is such that $q_{v}^{k}$ is idempotent for all $v \in V$. Note that by Corollary 53 this mapping is properly defined even if $\mu_{v}^{*} \neq \underline{0}_{v}$.

Lemma 71 Mappings $p_{v}^{\varphi}, v \in V$, are consistent.

Proof: Take any $C=\langle\mathbf{s}, R\rangle \in \mathcal{C}$. Since $\varphi$ is a solution of $\mathcal{P} / \bar{\mu}^{*}$, there is $\mathbf{b} \in R$ such that $\mathbf{b}[v] \in \varphi(v)$ for $v \in \mathbf{s}$. Then for any $\mathbf{a} \in R, q(\mathbf{a})=\mathbf{a} \cdot \mathbf{b} \in R$, and this product does not depend on the choice of $\mathbf{b}$, as it follows from Corollary 53 . Iterating this operation also produces a tuple from $R$.

We would like to use the above reduction to reduce $\mathcal{P}$ to a problem $\mathcal{P}^{\prime}$ such that $\operatorname{size}\left(\mathcal{P}^{\prime}\right)<\operatorname{size}(\mathcal{P})$. If $\varphi$ is such that for $v \in \operatorname{MAX}(\mathcal{P})$ there is $a \in \mathbb{A}_{v}$ with $a^{\mu_{v}^{*}} \leq \varphi(v)$ and $a \notin \varphi(v)$, then $\left|p_{v}^{\varphi}\left(\mathbb{A}_{v}\right)\right|<\left|\mathbb{A}_{v}\right|$. Also, observe that if $\left|p_{v}^{\varphi}\left(\mathbb{A}_{v}\right)\right|=\left|\mathbb{A}_{v}\right|$, then $p_{v}^{\varphi}$ is the identity mapping, that is $p_{v}^{\varphi}\left(\mathbb{A}_{v}\right)=\mathbb{A}_{v}$. If $\mathbb{A}_{v}$ is semilattice free then $p_{v}^{\varphi}$ is the identity mapping by Proposition 21. Let $V^{*}$ be the set of variables $v \in V$ such that $\mathbb{A}_{v} / \mu_{v}^{*}$ is not semilattice free.

Lemma 72 There are consistent mappings $p_{v}, v \in V$, such that for any $v \in V^{*}$ we have $\left|p_{v}\left(\mathbb{A}_{v}\right)\right|<\left|\mathbb{A}_{v}\right|$. Moreover, such mappings can be found solving a linear number of instances of the form $\left(\mathcal{P}_{\left(v, a^{\mu_{v}^{*}}\right)}\right) / \bar{\mu}^{*}$.

Proof: Since $\mathcal{P} / \bar{\mu}^{*}$ is globally 1 -minimal, for any $a \in \mathbb{A}_{v} / \mu_{v}^{*}$ there is a solution $\varphi$ with $\varphi(v)=a$, and it can be found solving the instance $\left(\mathcal{P}_{\left(v, a^{\mu *}\right)}\right) / \bar{\mu}^{*}$. For every $v \in V^{*}$ choose $a \in \mathbb{A}_{v}$ such that there is $b \in \mathbb{A}_{v}$ and $b \leq a, a$, and let $\varphi_{v}$ be a solution of $\mathcal{P} / \bar{\mu}^{*}$ with $\varphi_{v}(v)=a^{\mu_{v}^{*}}$. Then $\left|p_{v}^{\varphi_{v}}\left(\mathbb{A}_{v}\right)\right|<\left|\mathbb{A}_{v}\right|$ and $\left|p_{w}^{\varphi_{v}}\left(\mathbb{A}_{w}\right)\right|<\left|\mathbb{A}_{w}\right|$ or $p_{w}^{\varphi_{v}}$ is the identity mapping for any $w \in V^{*}$. Therefore the composition of the $p^{\varphi_{w}}$ for all $w \in V^{*}$ is as required.

Theorem 69 now follows by observing that if $\mathbb{A}_{v} / \mu_{v}^{*}$ is semilattice free then $\mathbb{A}_{v}$ itself is semilattice free.

In order to use Theorem 69 we however need to argue that $p(\mathcal{P})$ is a problem over a class of algebras omitting type 1 . Let $f$ be a weak near-unanimity term of the class $\mathcal{A}$. Then $p \circ f$ is a weak near-unanimity term of $p(\mathcal{A})=\{p(\mathbb{A}) \mid \mathbb{A} \in \mathcal{A}\}$. Moreover, if $\mathbb{A}$ is semilattice free then $p(\mathbb{A})=\mathbb{A}$. 


\subsection{Strategies}

In this section similar to strategies related to the concepts of consistency and minimality we introduce strategies of some sort that will be used to prove Theorem 68 . We start with some necessary definitions.

Let $\mathcal{P}=(V, \mathcal{C})$ be a $(2,3)$-minimal and block-minimal instance over $\mathcal{A}$. For $(v, \alpha, \beta) \in \mathcal{W}$, if $(v, \alpha, \beta) \notin \mathcal{W}^{\prime}$, then $\mathcal{S}_{W_{v, \alpha \beta, \bar{\beta}}}$ denotes the set of solutions of $\mathcal{P}_{W_{v, \alpha \beta, \bar{\beta}}}$, and if $(v, \alpha, \beta) \in \mathcal{W}^{\prime}$, then $\mathcal{S}_{W_{v, \alpha \beta, \bar{\beta}}, Y}$ denotes the set of solutions of $\mathcal{P}_{W_{v, \alpha \beta} \bar{\beta}} / \bar{\mu}^{Y}$ for an appropriate $Y$.

Let $\beta_{v} \in \operatorname{Con}\left(\mathbb{A}_{v}\right)$ and let $B_{v}$ be a $\beta_{v}$-block, $\bar{\beta}=\left(\beta_{v} \mid v \in V\right), \bar{B}=$ $\left(B_{v} \mid v \in V\right)$. Let $\mathcal{R}=\left\{R_{C, v, \alpha \beta} \mid C=\langle\mathbf{s}, R\rangle \in \mathcal{C},(v, \alpha, \beta) \in \mathcal{W}(\bar{\beta})\right\}$ be a collection of relations such that $R_{C, v, \alpha \beta}$ is a subalgebra of $\operatorname{pr}_{\mathbf{s} \cap W_{v, \alpha \beta, \bar{\beta}}} R$. Let $C=\langle\mathbf{s}, R\rangle,(v, \alpha, \beta) \in \mathcal{W}$, and $W=W_{v, \alpha \beta, \bar{\beta}}$. Let a be a tuple from $\operatorname{pr}_{X} R$ for $X \subseteq \mathbf{s}$, or from $\operatorname{pr}_{X} \mathcal{S}_{W}, X \subseteq W$, if $(v, \alpha, \beta) \notin \mathcal{W}^{\prime}$, or from $\operatorname{pr}_{X} \mathcal{S}_{W, Y}$ if $(v, \alpha, \beta) \in \mathcal{W}^{\prime}$, where $X \subseteq W$ and $Y$ is a set specified in the condition of blockminimality. Tuple a is said to be $\mathcal{R}$-compatible if for any $(w, \gamma, \delta) \in \mathcal{W}(\bar{\beta})$, (let $\left.U=W_{w, \gamma \delta, \bar{\beta}}\right) \operatorname{pr}_{X \cap U} \mathbf{a} \in \operatorname{pr}_{X \cap U} R_{C, w, \gamma \delta}$ or $\operatorname{pr}_{X \cap U} \mathbf{a} \in \operatorname{pr}_{X \cap U} R_{C, w, \gamma \delta} / \bar{\mu}^{Y}$ for an appropriate set $Y$. By $R^{\mathcal{R}}, \mathcal{S}_{W}^{\mathcal{R}}, \mathcal{S}_{W, Y}^{\mathcal{R}}$ we denote the set of all $\mathcal{R}$-compatible tuples from the corresponding relation. Also, let $\mathcal{P}^{\mathcal{R}}=\left(V, \mathcal{C}^{\mathcal{R}}\right)$ denote the problem instance obtained from $\mathcal{P}$ replacing every constraint $\langle\mathbf{s}, R\rangle \in \mathcal{C}$ with $\left\langle\mathbf{s}, R^{\mathcal{R}}\right\rangle$.

The collection $\mathcal{R}$ is called a $\bar{\beta}$-strategy with respect to $\bar{B}$ if it satisfies the following conditions for every $(v, \alpha, \beta) \in \mathcal{W}(\bar{\beta})$, and every $C=\langle\mathbf{s}, R\rangle \in \mathcal{C}$ (let $\left.W=W_{w, \alpha \beta, \bar{\beta}}\right)$ :

(S1) the relations $\operatorname{umax}\left(R^{X, \mathcal{R}}\right)$, where $R^{X, \mathcal{R}}$ consists of $\mathcal{R}$-compatible tuples from $R^{X}$ for $X \subseteq V,|X| \leq 2$, form a nonempty $(2,3)$-strategy for $\mathcal{P}^{\mathcal{R}}$;

(S2) for every $(w, \gamma, \delta) \in \mathcal{W}(\bar{\beta})\left(\right.$ let $\left.U=W_{w, \gamma \delta}\right)$ and every $\mathbf{a} \in \operatorname{umax}\left(\operatorname{pr}_{\mathbf{s} \cap W \cap U} R_{C, v, \alpha \beta}\right)$ it holds: if $(w, \gamma, \delta) \notin \mathcal{W}^{\prime}$ then a extends to an $\mathcal{R}$-compatible solution $\varphi$ of $\mathcal{P}_{U}$; otherwise if $(v, \alpha, \beta) \notin \mathcal{W}^{\prime}$ then a extends to an $\mathcal{R}$-compatible solution of $\mathcal{P}_{U} / \bar{\mu}^{Y_{1}}$ with $Y_{1}=\operatorname{MAX}(\mathcal{P})-(W \cap U)$; and if $(v, \alpha, \beta) \in \mathcal{W}^{\prime}$ then a extends to an $\mathcal{R}$-compatible solution of $\mathcal{P}_{U} / \bar{\mu}^{Y_{2}}$, where $Y_{2}=\operatorname{MAX}(\mathcal{P})-\mathbf{s}$;

(S3) $R \cap \bar{B}_{\mathbf{s}} \neq \varnothing$ and for any $I \subseteq \mathbf{s}$ any $\mathcal{R}$-compatible tuple $\mathbf{a} \in \operatorname{umax}\left(\operatorname{pr}_{I} R\right)$ extends to an $\mathcal{R}$-compatible tuple $\mathbf{b} \in R$.

(S4) the relation $R_{C, v, \alpha \beta}$ is a subalgebra of $\operatorname{pr}_{\mathbf{s} \cap W} R$, and $\operatorname{umax}\left(R_{C, v, \alpha \beta}\right) \subseteq$ $\operatorname{umax}\left(\operatorname{pr}_{\mathrm{s} \cap W} R\right)$; if $(v, \alpha, \beta) \notin \mathcal{W}^{\prime}$ then the relation $\mathcal{S}_{W}^{\mathcal{R}}$ is a subalgebra of $\mathcal{S}_{W}$, and $\operatorname{umax}\left(\mathcal{S}_{W}^{\mathcal{R}}\right) \subseteq \operatorname{umax}\left(\mathcal{S}_{W}\right)$; if $(v, \alpha, \beta) \in \mathcal{W}^{\prime}$ then for any $(w, \gamma, \delta) \in \mathcal{W}(\bar{\beta})-\mathcal{W}^{\prime}$ the relations $\mathcal{S}_{W, Y_{1}}^{\mathcal{R}}, \mathcal{S}_{W, Y_{2}}^{\mathcal{R}}$ are subalgebras of $\mathcal{S}_{W, Y_{1}}, \mathcal{S}_{W, Y_{2}}$, 
respectively, and $\operatorname{umax}\left(\mathcal{S}_{W, Y_{1}}^{\mathcal{R}}\right) \subseteq \operatorname{umax}\left(\mathcal{S}_{W, Y_{1}}\right), \operatorname{umax}\left(\mathcal{S}_{W, Y_{2}}^{\mathcal{R}}\right) \subseteq \operatorname{umax}\left(\mathcal{S}_{W, Y_{2}}\right)$, where $Y_{1}=\operatorname{MAX}(\mathcal{P})-\mathbf{s}$ and $Y_{2}=\operatorname{MAX}(\mathcal{P})-\left(W \cap W_{w, \gamma \delta}\right)$;

(S5) for every $w \in \mathbf{s}$ and every $(w, \gamma, \delta) \in \mathcal{W}(\bar{\beta})$ (let $\left.U=W_{w, \gamma \delta}\right)$ with $w \in$ $\mathbf{s} \cap U$ it holds umax $\left(\operatorname{pr}_{w} R_{C, w, \gamma \delta}\right)=\operatorname{umax}\left(\operatorname{pr}_{w} R_{C, v, \alpha \beta}\right)$, let $A_{\mathcal{R}, w}$ denote the subalgebra generated by this set, $\operatorname{umax}\left(A_{\mathcal{R}, w}\right)$ is as-closed in $\operatorname{umax}\left(\operatorname{pr}_{w}(R \cap\right.$ $\bar{B})$;

(S6) for every $(w, \gamma, \delta) \in \mathcal{W}(\bar{\beta})$ with $\mathbf{s} \cap W_{w, \gamma \delta} \neq \varnothing$ the set of $\mathcal{R}$-compatible tuples from $R_{C, w, \gamma \delta}$ is polynomially closed in $\operatorname{pr}_{\mathbf{s} \cap W_{w, \gamma \delta}} R$;

(S7) relation $R$ is strongly chained with respect to $\bar{\beta}, \bar{B}$; if $(v, \alpha, \beta) \notin \mathcal{W}^{\prime}$, relation $\mathcal{S}_{W}$ is strongly chained with respect to $\bar{\beta}, \bar{B}$; if $(v, \alpha, \beta) \in \mathcal{W}^{\prime}$, for any $(w, \gamma, \delta) \in \mathcal{W}(\bar{\beta})-\mathcal{W}^{\prime}$ the relations $\mathcal{S}_{W, Y_{1}}, \mathcal{S}_{W, Y_{2}}, Y_{1}=\operatorname{MAX}(\mathcal{P})-\mathbf{s}$, $Y_{2}=\operatorname{MAX}(\mathcal{P})-\left(W \cap W_{w, \gamma \delta}\right)$, are strongly chained with respect to $\bar{\beta}, \bar{B}$.

Conditions (S1)-(S3) are the conditions we actually want to maintain when transforming a strategy, and these are the ones that provide the desired results. However, to prove that (S1)-(S3) are preserved under transformations of a strategy we also need more technical conditions (S4)-(S7).

We now show how we plan to use $\bar{\beta}$-strategies. Let $\mathcal{P}$ be a subdirectly irreducible, (2,3)-minimal, and block-minimal instance, $\beta_{v}=\underline{1}_{v}$ and $B_{v}=\mathbb{A}_{v}$ for $v \in V$. Then as is easily seen the collection of relations $\mathcal{R}=\left\{R_{C, v, \alpha \beta} \mid\right.$ $(v, \alpha, \beta) \in \mathcal{W}(\bar{\beta}), C \in \mathcal{C}\}$ given by $R_{C, v, \alpha \beta}=\operatorname{pr}_{\mathbf{s} \cap W_{v, \alpha \beta, \bar{\beta}}} R$ for $C=\langle\mathbf{s}, R\rangle \in \mathcal{C}$ is a $\bar{\beta}$-strategy with respect to $\bar{B}$. Also, by (S3) a $\bar{\gamma}$-strategy with $\gamma_{v}=\underline{0}_{v}$ for all $v \in V$ gives a solution of $\mathcal{P}$. Our goal is therefore to show that a $\bar{\beta}$-strategy for any $\bar{\beta}$ can be 'reduced', that is, transformed to a $\bar{\beta}^{\prime}$-strategy for some $\bar{\beta}^{\prime}<\bar{\beta}$. Note that this reduction of strategies is where the condition $\operatorname{MAX}(\mathcal{P}) \cap \operatorname{Center}(\mathcal{P})=\varnothing$ is used. Indeed, suppose that $\beta_{v}=\mu_{v}^{*}$. Then by conditions (S1)-(S7) we only have information about solutions to problems of the form $\mathcal{P}_{W} / \bar{\mu}^{*}$ or something very close to that. Therefore this barrier cannot be penetrated. We consider two cases.

CASE 1 . There are $v \in V$ and $\alpha \prec \beta_{v}$ nontrivial on $B_{v}$, typ $\left(\alpha, \beta_{v}\right)=\mathbf{2}$. This case is considered in Section 10.1 .

CASE 2. For all $v \in V$ and $\alpha \prec \beta_{v}$ nontrivial on $B_{v}$, typ $\left(\alpha, \beta_{v}\right) \in\{\mathbf{3}, \mathbf{4}, \mathbf{5}\}$. This case is considered in Section 10.2 .

\section{Proof of Theorem 68}

In the remaining part of the paper we prove Theorem 68 


\subsection{Tightening affine factors}

In this section we consider Case 1 of tightening strategies: there is $\alpha \in \operatorname{Con}\left(\mathbb{A}_{v}\right)$ for some $v \in V$ such that $\alpha \prec \beta_{v}$ and typ $\left(\alpha, \beta_{v}\right)=\mathbf{2}$.

\subsubsection{Transformation of the strategy and induced congruences}

Let $\mathcal{P}=(V, \mathcal{C})$ be a block-minimal instance with subdirectly irreducible domains, $\bar{\beta}=\left(\beta_{v} \in \operatorname{Con}\left(\mathbb{A}_{v}\right) \mid v \in V\right)$ and $\bar{B}=\left(B_{v} \mid B_{v}\right.$ is a $\beta_{v}$-block, $\left.v \in V\right)$. We use notation from Section 9 . Let also $\mathcal{R}=\left\{R_{C, v, \alpha \beta}\right\}$ be a $\bar{\beta}$-strategy for $\bar{B}$. We select $v \in V$ and $\alpha, \beta \in \operatorname{Con}\left(\mathbb{A}_{v}\right)$ with $\alpha \prec \beta=\beta_{v}$, typ $(\alpha, \beta)=\mathbf{2}$, and an $\alpha$-block $B \in B_{v} / \alpha$. Note that since $\operatorname{typ}(\alpha, \beta)=\mathbf{2}, B_{v} / \alpha$ is a module, and therefore $B_{v}^{\prime}$ is as-maximal in this set. In this section we show how $\mathcal{R}$ can be transformed to a $\bar{\beta}^{\prime}$-strategy $\mathcal{R}^{\prime}$ for $\bar{B}^{\prime}$ such that $\beta_{w}^{\prime} \leq \beta_{w}, B_{w}^{\prime} \subseteq B_{w}$ for $w \in V$, and $\beta_{v}^{\prime}=\alpha$, $B_{v}^{\prime}=B$.

First of all we identify variables $w \in V$ for which $\beta_{w}^{\prime}$ has to be different from $\beta_{w}$. Since $\mathcal{P}$ is $(2,3)$-minimal, for every $u, w \in V$ there is $C^{\{u, w\}}=$ $\left\langle(u, w), R^{\{u, w\}}\right\rangle \in \mathcal{C}$. For $w \in W_{v, \alpha \beta}$ (we omit $\bar{\beta}$ from $W_{v, \alpha \beta, \bar{\beta}}$ here) consider $R^{*,\{v, w\}}=R^{\{v, w\}} \cap\left(B_{v} \times B_{w}\right), R^{\{v, w\}, \mathcal{R}}$ the set of all $\mathcal{R}$-compatible pairs from $R^{\{v, w\}}, R^{\prime\{v, w\}}=R^{\{v, w\}} / \alpha$, and $R^{\prime\{v, w\}, \mathcal{R}}=R^{\{v, w\}, \mathcal{R}} / \alpha$. By (S5) for $\mathcal{R}$ we have that $\operatorname{umax}\left(\operatorname{pr}_{v} R^{\prime\{v, w\}, \mathcal{R}}\right)$ is as-closed in $B_{v}^{*} / \alpha$, where $B_{v}^{*}=\operatorname{pr}_{v} R^{*,\{v, w\}}$; since $\operatorname{typ}(\alpha, \beta)=\mathbf{2}$, this implies $\operatorname{pr}_{v} R^{\prime\{v, w\}, \mathcal{R}}=B_{v}^{*} / \alpha$. Also, $\operatorname{pr}_{v} R^{\{v, w\}, \mathcal{R}}$, and therefore $\operatorname{pr}_{v} R^{\prime\{v, w\}, \mathcal{R}}$ are polynomially closed in $\operatorname{pr}_{v} R^{\{v, w\}}, \operatorname{pr}_{v} R^{\prime\{v, w\}}$, respectively, by (S6), and $\operatorname{pr}_{v} R^{\{v, w\}}, \operatorname{pr}_{v} R^{\prime\{v, w\}}$ are strongly chained by (S7). Therefore, by the Congruence Lemma64 either $B_{v}^{*} / \alpha \times \operatorname{umax}\left(\operatorname{pr}_{w}\left(R^{\prime\{v, w\}, \mathcal{R}}\right) \subseteq R^{\prime\{v, w\}, \mathcal{R}}\right.$ or $R^{\prime\{v, w\}, \mathcal{R}}$ is the graph of a mapping $\nu_{w}: \operatorname{pr}_{w} R^{\prime\{v, w\}, \mathcal{R}} \rightarrow B_{v}^{*} / \alpha$. Let $U \subseteq W_{v, \alpha \beta}$ be the set of variables for which the latter holds, and let $\alpha_{w}$ be the corresponding congruence of $\mathbb{A}_{w}$, extension of the kernel of $\nu_{w}$. Let $\beta_{v}^{\prime}=\alpha, B_{v}^{\prime}=B$ and $\beta_{w}^{\prime}=\alpha_{w}, B_{w}^{\prime}=\nu_{w}^{-1}(B)$ for $w \in U$, and $\beta_{w}^{\prime}=\beta_{w}, B_{w}^{\prime}=B_{w}$ for $w \in V-U$.

Now we are in a position to define the new strategy. Let $\mathcal{R}^{\prime}$ be the following collection of relations. We omit subscript $\bar{\beta}$.

(R1) $\mathcal{R}^{\prime}=\left\{R_{C, w, \gamma \delta}^{\prime} \mid C=\langle\mathbf{s}, R\rangle \in \mathcal{C},(w, \gamma, \delta) \in \mathcal{W}\left(\bar{\beta}^{\prime}\right)\right\}$;

(R2) for every $C=\langle\mathbf{s}, R\rangle \in \mathcal{C},(u, \gamma, \delta) \in \mathcal{W}\left(\bar{\beta}^{\prime}\right)$,

(a) if $(v, \alpha, \beta) \notin \mathcal{W}^{\prime}, R_{C, u, \gamma \delta}^{\prime}=\left\{\mathbf{a} \in R_{C, u, \gamma \delta} \mid\right.$ there is a $\mathcal{R}$-compatible solution $\varphi$ of $\mathcal{P}_{W_{v, \alpha \beta}}, \varphi(v) \in B_{v}^{\prime}$, and $\varphi(w)=\mathbf{a}[w]$ for $w \in \mathbf{s} \cap$ $\left.W_{v, \alpha \beta_{v}} \cap W_{u, \gamma \delta}\right\}$;

(b) if $(v, \alpha, \beta) \in \mathcal{W}^{\prime},(u, \gamma, \delta) \notin \mathcal{W}^{\prime}, R_{C, u, \gamma \delta}^{\prime}=\left\{\mathbf{a} \in R_{C, u, \gamma \delta} \mid\right.$ there is a $\mathcal{R}$-compatible solution $\varphi$ of $\mathcal{P}_{W_{v, \alpha \beta v}} / \bar{\mu}^{Y}$ with $Y=\operatorname{MAX}(\mathcal{P})-W_{u, \gamma \delta}$, 
such that $\varphi(v) \in B_{v}^{\prime} / \mu_{v}^{Y}$, and $\varphi(w)=\mathbf{a}[w]$ for $w \in \mathbf{s} \cap W_{v, \alpha \beta_{v}} \cap$ $\left.W_{u, \gamma \delta}\right\}$

(c) if $(v, \alpha, \beta),(u, \gamma, \delta) \in \mathcal{W}^{\prime}, R_{C, u, \gamma \delta}^{\prime}=\left\{\mathbf{a} \in R_{C, u, \gamma \delta} \mid\right.$ there is a $\mathcal{R}$ compatible solution $\varphi$ of $\mathcal{P}_{W_{v, \alpha \beta v}} / \bar{\mu}^{Y}$ with $Y=\operatorname{MAX}(\mathcal{P})-\mathbf{s}$, such that $\varphi(v) \in B_{v}^{\prime} / \mu_{v}^{Y}$, and $\varphi(w)=\mathbf{a}[w]$ for $\left.w \in \mathbf{s} \cap W_{v, \alpha \beta_{v}} \cap W_{u, \gamma \delta}\right\}$;

Similar to $R^{\mathcal{R}}, \mathcal{S}_{W}^{\mathcal{R}}, \mathcal{S}_{W, Y}^{\mathcal{R}}$ by $R^{\mathcal{R}^{\prime}}, \mathcal{S}_{W}^{\mathcal{R}^{\prime}}, \mathcal{S}_{W, Y}^{\mathcal{R}^{\prime}}$ we denote the corresponding sets of $\mathcal{R}^{\prime}$-compatible tuples. As is easily seen, the sets of both types are indeed subalgebras of $R, \mathcal{S}_{W}, \mathcal{S}_{W, Y}$.

The following three statements show how relations $R_{C, w, \gamma \delta}^{\prime}$ from $\mathcal{R}^{\prime}$ are related to $R_{C, w, \gamma \delta}$ from $\mathcal{R}$. They amount to saying that either $R_{C, w, \gamma \delta}^{\prime}$ is the intersection of $R_{C, w, \gamma \delta}$ with a block of a congruence of the projection of $R$, or umax $\left(R_{C, w, \gamma \delta}^{\prime}\right)=$ $\operatorname{umax}\left(R_{C, w, \gamma \delta}\right)$. Recall that for congruences $\beta_{w}, w \in V$, and $U \subseteq V$ by $\bar{\beta}_{U}$ we denote the collection $\left(\beta_{w}\right)_{U \subseteq V}$. Set $W=W_{v, \alpha \beta}$ for the rest of Section 10.1 .

Lemma 73 Let $C=\langle\mathbf{s}, R\rangle \in \mathcal{C}$, and let $\mathcal{S}_{W}^{\prime}$ be the set of solutions of $\mathcal{P}_{W}$ if $(v, \alpha, \beta) \notin \mathcal{W}^{\prime}$, or the set of solutions of $\mathcal{P}_{W} / \bar{\mu}^{\operatorname{Max}(\mathcal{P})-\mathrm{s}}$ if $(v, \alpha, \beta) \in \mathcal{W}^{\prime}$. For every $U \subseteq \mathbf{s} \cap W$ there is a congruence $\tau_{U}$ of $\operatorname{pr}_{U} \mathcal{S}_{W}^{\prime}=\operatorname{pr}_{U} R$ satisfying the following conditions:

(1) either umax $\left(\operatorname{pr}_{U} \mathcal{S}_{W}^{\prime \mathcal{R}^{\prime}}\right)=\operatorname{umax}\left(\operatorname{pr}_{U} \mathcal{S}_{W}^{\prime \mathcal{R}}\right)$ or for a $\tau_{U}$-block $S$ it holds $\operatorname{pr}_{U} \mathcal{S}_{W}^{\prime \mathcal{R}^{\prime}}=$ $\operatorname{pr}_{U} \mathcal{S}_{W}^{\prime \mathcal{R}} \cap S$.

(2) For any $U_{1} \subseteq U_{2} \subseteq W$ the congruence $\tau_{U_{1}}$ is the restriction of $\tau_{U_{2}}$, that is $(\mathbf{a}, \mathbf{b}) \in \tau_{U_{1}}$ if and only if for some $\mathbf{a}^{\prime}, \mathbf{b}^{\prime} \in \mathcal{S}_{U_{2}}^{\prime}$ with $\operatorname{pr}_{U_{1}} \mathbf{a}^{\prime}=\mathbf{a}, \operatorname{pr}_{U_{1}} \mathbf{b}^{\prime}=\mathbf{b}$ it holds $\left(\mathbf{a}^{\prime}, \mathbf{b}^{\prime}\right) \in \tau_{U_{2}}$.

(3) For any $U \subseteq \mathbf{s} \cap W$ either $\left.\tau_{U}\right|_{\mathrm{pr}_{U} R \cap \bar{B}}=\left.\bar{\beta}_{U}\right|_{\mathrm{pr}_{U} R \cap \bar{B}}$, or the algebra $\operatorname{pr}_{U} \mathcal{S}_{W}^{\prime \mathcal{R}} / \tau_{U}$ is isomorphic to $\operatorname{pr}_{v}\left(\mathcal{S}_{W}^{\prime} \cap \bar{B}\right) / \alpha$.

(4) For any $(w, \gamma, \delta) \in \mathcal{W}(\bar{\beta}), X=W_{w, \gamma \delta}, X^{\prime}=\mathbf{s} \cap W \cap X$, let $\tau=\tau_{X^{\prime}}$. Then either $\operatorname{umax}\left(\operatorname{pr}_{X^{\prime}} R_{C, w, \gamma \delta}^{\prime}\right)=\operatorname{umax}\left(\operatorname{pr}_{X^{\prime}} R_{C, w, \gamma \delta}\right)$, or for a $\tau$-block $S$ it holds $\operatorname{pr}_{X^{\prime}} R_{C, w, \gamma \delta}^{\prime} \subseteq \operatorname{pr}_{X^{\prime}} R_{C, w, \gamma \delta} \cap S$ and $\operatorname{umax}\left(\operatorname{pr}_{X^{\prime}} R_{C, w, \gamma \delta}^{\prime}\right)$ is the set of u-maximal elements of $\operatorname{umax}\left(\operatorname{pr}_{X^{\prime}} R_{C, w, \gamma \delta}\right) \cap S$.

If, according to item (3) of the lemma, $\left.\tau_{U}\right|_{p_{U} R \cap \bar{B}}=\left.\bar{\beta}_{U}\right|_{p_{U} R \cap \bar{B}}$, we say that $\tau_{U}$ is the full congruence; if the latter option of item (3) holds we say that $\tau_{U}$ is a maximal congruence.

Proof: If $v \in U$ then set $\tau_{U}$ to be $\bar{\beta}_{U}^{\prime}$. Otherwise consider $Q=\operatorname{pr}_{U \cup\{v\}} \mathcal{S}_{W}^{\prime}$ as a subdirect product of $\mathbb{A}_{v}$ and $\operatorname{pr}_{U} \mathcal{S}_{W}^{\prime}$. This relation is chained by (S7) and $\operatorname{pr}_{U \cap\{v\}} \mathcal{S}_{W}^{\prime \mathcal{R}}$ is polynomially closed in $Q$ by Lemma 61(2); apply the Congruence Lemma 64 to it. Specifically, consider $Q / \alpha$ as a subdirect product of $\operatorname{pr}_{U} \mathcal{S}_{W}^{\prime}$ and $\mathbb{A}_{v} / \alpha$. If the first option of the Congruence Lemma 64 holds, set $\tau_{U}=\bar{\beta}_{U}$. If the 
second option is the case, choose $\tau_{U}$ to the congruence $\eta$ of $\operatorname{pr}_{U} \mathcal{S}_{W}^{\prime}$ identified in the Congruence Lemma 64 .

(1) In this case the result follows by the Congruence Lemma 64

(2) Obvious.

(3) If $v \in U$ then by item (1) $\operatorname{pr}_{U} \mathcal{S}_{W}^{\prime \mathcal{R}} / \tau_{U}=\operatorname{pr}_{U} \mathcal{S}_{W}^{\prime \mathcal{R}} / \bar{\beta}_{U}^{\prime}$, which is isomorphic to $\operatorname{pr}_{v}\left(\mathcal{S}_{W}^{\prime} \cap \bar{B}\right) / \alpha$. Otherwise consider relation $Q$ as in the beginning of the proof. By the Congruence Lemma 64 if $\tau_{U} \neq \bar{\beta}_{U}, \tau_{U} \prec \bar{\beta}_{U}$. The result follows.

(4) If $\tau$ is the full congruence then by (S2) for $\mathcal{R}$ we have umax $\left(\operatorname{pr}_{X} R_{C, w, \gamma \delta}\right)=$ $\operatorname{umax}\left(\operatorname{pr}_{X} \mathcal{S}_{W}^{\prime \mathcal{R}^{\prime}}\right)$ and we have the first option. If $\tau$ is a maximal congruence then by (R2) and item (1) there is a $\tau$-block $S$ such that $\operatorname{pr}_{X^{\prime}} R_{C, w, \gamma \delta}^{\prime} \subseteq S \cap \operatorname{pr}_{X^{\prime}} R_{C, w, \gamma \delta}$. By condition (S2) every a $\in \operatorname{umax}\left(\operatorname{pr}_{X^{\prime}} R_{C, w, \gamma \delta}\right)$ can be extended to a solution from $\mathcal{S}_{W}^{\mathcal{R}}$. In particular, if a $\in \operatorname{umax}\left(\mathrm{pr}_{X^{\prime}} R_{C, w, \gamma \delta}^{\prime}\right) \subseteq S$ then such an extension $\varphi$ has to satisfy $\varphi(v) \in B_{v}^{\prime}$. Since $S$ is as-maximal in $\operatorname{pr}_{X^{\prime}}\left(R_{C, w, \gamma \delta}\right) / \tau$ and $\operatorname{umax}\left(\operatorname{pr}_{X^{\prime}} R_{C, w, \gamma \delta}^{\prime}\right) \subseteq \operatorname{umax}\left(\operatorname{pr}_{X^{\prime}} R_{C, w, \gamma \delta}\right)$, we have $\operatorname{umax}\left(\operatorname{pr}_{X^{\prime}} R_{C, w, \gamma \delta}^{\prime}\right)=$ $\operatorname{umax}\left(S \cap \operatorname{pr}_{X^{\prime}} R_{C, w, \gamma \delta}\right)$.

For $C=\langle\mathbf{s}, R\rangle \in \mathcal{C}$ we use $\tau_{C}$ to denote the congruence $\tau_{\mathbf{s} \cap W}$. Also for $(w, \gamma, \delta) \in \mathcal{W}(\bar{\beta})$ we use $\tau_{C, w, \gamma \delta}$ to denote the congruence $\tau_{\mathbf{s} \cap W \cap W_{w, \gamma \delta}}$.

Lemma 74 In the notation above let $\gamma_{u}, \delta_{u} \in \operatorname{Con}\left(\mathbb{A}_{u}\right), u \in W^{\prime}=\mathbf{s} \cap W$ be such that $\left(u, \gamma_{u}, \delta_{u}\right) \in \mathcal{W}(\bar{\beta})$ and $\left(\alpha, \beta_{v}\right),\left(\gamma_{u}, \delta_{u}\right)$ cannot be separated from each other. Then if $\tau_{C}$ is a maximal congruence, for any polynomial $f$ of $R, f\left(\bar{\beta}_{W^{\prime}}\right) \subseteq \tau_{C}$ if and only if $f\left(\delta_{u}\right) \subseteq \gamma_{u}$ for any $u \in W^{\prime}$. If $\gamma_{u}, \delta_{u}$ are considered as congruences of $\mathrm{pr}_{W^{\prime}} R$, equal to $\gamma_{u} \times \prod_{x \in W^{\prime}-\{u\}} \underline{1}_{x}, \delta_{u} \times \prod_{x \in W^{\prime}-\{u\}} \underline{1}_{x}$, respectively, this condition means that $\left(\tau_{C}, \bar{\beta}_{W^{\prime}}\right)$ and $\left(\gamma_{u}, \delta_{u}\right)$ cannot be separated.

Proof: Let $\mathcal{S}_{W}^{\prime}$ be defined as in Lemma 73 and $\tau_{C}$ a maximal congruence. Take a polynomial $f$ of $R$. As $\left(\gamma_{u_{1}}, \delta_{u_{1}}\right),\left(\gamma_{u_{2}}, \delta_{u_{2}}\right)$ cannot be separated for any $u_{1}, u_{2} \in W^{\prime}$, it suffices to consider just one variable $u \in W^{\prime}$. Since $\mathcal{P}$ is a blockminimal instance, the polynomial $f$ can be extended from a polynomial on $\mathrm{pr}_{W^{\prime}} R$ to a polynomial $f^{\prime}$ of $\mathcal{S}_{W}^{\prime}$, and, in particular, to a polynomial $f^{\prime \prime}$ of $\operatorname{pr}_{W^{\prime} \cup\{v\}} \mathcal{S}_{W}^{\prime}$. Since $\tau_{C}$ is maximal, by the Congruence Lemma 64 the intervals $\left(\alpha, \beta_{v}\right)$ and $\left(\tau_{C}, \bar{\beta}_{W^{\prime}}\right)$ in the congruence lattices of $\mathbb{A}_{v}$ and $\operatorname{pr}_{W^{\prime}} R$, respectively, cannot be separated in $\operatorname{pr}_{W^{\prime} \cup\{v\}} \mathcal{S}_{W}^{\prime}$. Therefore $f^{\prime \prime}\left(\beta_{v}\right) \subseteq \alpha$ if and only if $f\left(\bar{\beta}_{W^{\prime}}\right) \subseteq \tau_{C}$. Since $\left(\alpha, \beta_{v}\right)$ and $\left(\gamma_{u}, \delta_{u}\right)$ cannot be separated in $\mathcal{P}$, the first inclusion holds if and only if $f\left(\delta_{u}\right) \subseteq \gamma_{u}$, and we infer the result.

Corollary 75 For any $(w, \gamma, \delta) \in \mathcal{W}(\bar{\beta}), X=\mathbf{s} \cap W_{w, \gamma \delta}, X^{\prime}=W \cap X$, let $\tau=\tau_{X^{\prime}}, \tau^{\prime}=\left\{(\mathbf{a}, \mathbf{b}) \mid \mathbf{a}, \mathbf{b} \in \operatorname{pr}_{X} R,\left(\operatorname{pr}_{X^{\prime}} \mathbf{a}, \operatorname{pr}_{X^{\prime}} \mathbf{b}\right) \in \tau\right\}$, and $\tau^{\prime}=$ $\left\{(\mathbf{a}, \mathbf{b}) \mid \mathbf{a}, \mathbf{b} \in \operatorname{pr}_{X} R,\left(\operatorname{pr}_{X^{\prime}} \mathbf{a}, \operatorname{pr}_{X^{\prime}} \mathbf{b}\right) \in \tau, \operatorname{pr}_{X-X^{\prime}} \mathbf{a}=\operatorname{pr}_{X-X^{\prime}} \mathbf{b}\right\}$. Then either $\operatorname{umax}\left(R_{C, w, \gamma \delta}^{\prime}\right)=\operatorname{umax}\left(R_{C, w, \gamma \delta}\right)$, or for a $\tau^{\prime}$-block $T$ it holds $R_{C, w, \gamma \delta}^{\prime} \subseteq$ 
$R_{C, w, \gamma \delta} \cap T$ and $\operatorname{umax}\left(R_{C, w, \gamma \delta}^{\prime}\right)$ is the set of $u$-maximal elements of $\operatorname{umax}\left(R_{C, w, \gamma \delta}\right) \cap$ T. Moreover, $S \times \operatorname{umax}\left(\operatorname{pr}_{X-X^{\prime}} R_{C, w, \gamma \delta}\right) \subseteq R_{C, w, \gamma \delta} / \tau^{\prime \prime}$.

Proof: By Lemma 73(4) either umax $\left(\operatorname{pr}_{X^{\prime}} R_{C, w, \gamma \delta}^{\prime}\right)=\operatorname{umax}\left(\operatorname{pr}_{X^{\prime}} R_{C, w, \gamma \delta}\right)$, or for a $\tau$-block $S$ it holds $\operatorname{pr}_{X^{\prime}} R_{C, w, \gamma \delta}^{\prime} \subseteq \operatorname{pr}_{X^{\prime}} R_{C, w, \gamma \delta} \cap S$ and umax $\left(\operatorname{pr}_{X^{\prime}} R_{C, w, \gamma \delta}^{\prime}\right)$ is the set of u-maximal elements of $\operatorname{umax}\left(\operatorname{pr}_{X^{\prime}} R_{C, w, \gamma \delta}\right) \cap S$. Then considering $R_{C, w, \gamma \delta} / \tau^{\prime \prime}$ as a subdirect product of $\operatorname{pr}_{X^{\prime}} R_{C, w, \gamma \delta} / \tau$ and $\operatorname{pr}_{X-X^{\prime}} R_{C, w, \gamma \delta}$, the interval $\left(\tau, \bar{\beta}_{X^{\prime}}\right)$ in $\operatorname{pr}_{X^{\prime}} R / \tau$ can be separated from interval $(\eta, \theta)$ in Con $\left(\mathbb{A}_{u}\right)$ for any $u \in X-X^{\prime}$ by Lemma 74. Then we use the Congruence Lemma 64 to conclude that $S \times \operatorname{umax}\left(\operatorname{pr}_{X-X^{\prime}} R_{C, w, \gamma \delta}\right) \subseteq R_{C, w, \gamma \delta} / \tau^{\prime \prime}$. The result follows.

Now we are in a position to prove that $\mathcal{R}^{\prime}$ is a $\bar{\beta}^{\prime}$-strategy.

Theorem 76 In the notation above, $\mathcal{R}^{\prime}$ is a $\bar{\beta}^{\prime}$-strategy for $\bar{B}^{\prime}$.

We give proofs of most difficult conditions from (S1)-(S7) in separate lemmas.

\subsubsection{Condition (S6)}

We start with condition (S6), as it will be needed for other conditions.

Lemma 77 Condition (S6) for $\mathcal{R}^{\prime}$ holds. That is, for every $(w, \gamma, \delta) \in \mathcal{W}(\bar{\beta})$ with $\mathbf{s} \cap W_{w, \gamma \delta} \neq \varnothing$ the set of $\mathcal{R}^{\prime}$-compatible tuples from $R_{C, w, \gamma \delta}$ is polynomially closed in $\operatorname{pr}_{\mathbf{s} \cap W_{w, \gamma \delta}} R$.

Proof: Consider $C=\langle\mathbf{s}, R\rangle \in \mathcal{C}$. Let $f$ be a polynomial of $R$, and let $\mathbf{a}, \mathbf{b} \in R$ be tuples satisfying the conditions of polynomial closeness. Let $\mathbf{c} \in \operatorname{Sg}(\mathbf{a}, f(\mathbf{b}))$ be such that a $\sqsubseteq_{a s} \mathbf{c}$ in $\operatorname{Sg}(\mathbf{a}, f(\mathbf{b}))$. By (S6) for $\mathcal{R}$, $\mathbf{c}$ is $\mathcal{R}$-compatible. It suffices to show that $\operatorname{pr}_{\mathbf{s} \cap W} \mathbf{c}$ is in the same $\tau_{C}$ block as $\operatorname{pr}_{\mathbf{s} \cap W} \mathbf{a}$. However, this is straightforward, because $\operatorname{pr}_{\mathbf{s} \cap W} \mathbf{a} \stackrel{\tau_{C}}{\equiv} \operatorname{pr}_{\mathbf{s} \cap W} \mathbf{b}$, and as $f(\mathbf{a})=\mathbf{a}$, we also have $\operatorname{pr}_{\mathbf{s} \cap W} \mathbf{a} \stackrel{\tau_{C}}{=} f\left(\operatorname{pr}_{\mathbf{s} \cap W} \mathbf{b}\right)$. Since $\operatorname{pr}_{\mathbf{s} \cap W} \mathbf{c} \in \operatorname{Sg}\left(\operatorname{pr}_{\mathbf{s} \cap W} \mathbf{a}, f\left(\operatorname{pr}_{\mathbf{s} \cap W} \mathbf{b}\right)\right)$, it follows $\mathrm{pr}_{\mathbf{s} \cap W} \mathbf{c} \stackrel{\tau_{C}}{=} \operatorname{pr}_{\mathbf{s} \cap W} \mathbf{a}$.

\subsubsection{Condition (S1)}

In this section we prove

Lemma 78 Condition (S1) for $\mathcal{R}^{\prime}$ holds. That is, the relations umax $\left(R^{X, \mathcal{R}^{\prime}}\right)$, where $R^{X, \mathcal{R}^{\prime}}$ consists of $\mathcal{R}^{\prime}$-compatible tuples from $R^{X}$ for $X \subseteq V,|X| \leq 2$, form a nonempty $(2,3)$-strategy for $\mathcal{P}^{\mathcal{R}^{\prime}}$.

We start with an auxiliary lemma. 
Lemma 79 Let $\mathbb{A}$ be an algebra and $\beta \in \operatorname{Con}(\mathbb{A})$ and $\mathbb{A}^{\prime}$ a subalgebra of a $\beta$ block. Let also $\alpha<\left.\beta\right|_{\mathbb{A}^{\prime}}$ be a congruence of $\mathbb{A}^{\prime}$ such that $\mathbb{A}^{\prime} / \alpha$ is a module. Let $\mathbb{B}, \mathbb{C}$ be subalgebras of $\mathbb{A}^{\prime}$ such that $\mathbb{B} \cap \mathbb{C} \neq \varnothing$ and $\mathbb{B} \cap \mathbb{C}$ contains a u-maximal element of an $\alpha$-block of $\mathbb{A}^{\prime}, \mathbb{B} / \alpha=\mathbb{A}^{\prime} /{ }_{\alpha}, \mathbb{C} / \alpha=\mathbb{A}^{\prime} / \alpha$, and $\mathbb{B}, \mathbb{C}$ are polynomially closed in $\mathbb{A}$. Then $(\mathbb{B} \cap \mathbb{C}) / \alpha=\mathbb{A}^{\prime} / \alpha$.

Proof: Let $a \in \mathbb{B} \cap \mathbb{C}$ be u-maximal in an $\alpha$-block of $\mathbb{A}^{\prime}$. As $\mathbb{A}^{\prime} / \alpha$ is a module, by Lemma 26 it is u-maximal in the $\gamma$-block $a^{\gamma}$ for any $\gamma \in \operatorname{Con}\left(\mathbb{A}^{\prime}\right), \alpha \leq \gamma$. Let $a^{\prime} \in \mathbb{B}$ and $a^{\prime} \not{p} a$; let also $\delta=\operatorname{Cg}\left(\left\{\left(a, a^{\prime}\right)\right\} \cup \alpha\right)$ in $\mathbb{A}^{\prime}$ and $\gamma \in \operatorname{Con}\left(\mathbb{A}^{\prime}\right)$ any such that $\alpha \leq \gamma \prec \delta$. For any $(\gamma, \delta)$-trace $T$ that contains $a$ and any $b \in T$, $a^{\gamma} \neq b^{\gamma}$, there is a polynomial $f$ of $\mathbb{A}$ such that $f(a)=a$ and $f\left(a^{\prime}\right)=b$. Since $\mathbb{B}$ is polynomially closed, for any $c \in \operatorname{Sg}(a, b)$ such that $a \sqsubseteq a s c$, we have $c \in \mathbb{B}$, and $c$ is u-maximal in $\mathbb{A}^{\prime}$. In a similar way $c \in \mathbb{C}$. Therefore it suffices to show that for any $\delta=\operatorname{Cg}\left(\left\{\left(a, a^{\prime}\right)\right\} \cup \alpha\right)$ in $\mathbb{A}^{\prime}$ for some $a^{\prime} \in \mathbb{A}^{\prime}$ and any $\gamma \in \operatorname{Con}\left(\mathbb{A}^{\prime}\right)$, $\alpha \leq \gamma \prec \delta$ there is $b \in \mathbb{A}^{\prime}$ such that $\{a, b\}$ is a $(\gamma, \delta)$-subtrace. Indeed, as is proved above $b \in \mathbb{B} \cap \mathbb{C}$, and therefore $(\mathbb{B} \cap \mathbb{C}) / \alpha$ is not contained in a $\delta$-block. Since $\mathbb{A}^{\prime} / \alpha$ is a module, this means $(\mathbb{B} \cap \mathbb{C}) / \alpha=\mathbb{A}^{\prime} / \alpha$.

Since $\mathbb{A}^{\prime} / \alpha$ is a module, there is a $(\gamma, \delta)$-subtrace $\{d, e\}$ such that $d \stackrel{\gamma}{=} a$. Then by Lemma 57 there is also a polynomial $g$ such that $g(\mathbb{A})$ is an $(\gamma, \delta)$-minimal set and $g(a)=a$. The result follows.

We now can prove Lemma 78

Proof:(of Lemma 78) We consider the collection of constraints $C^{X}=$ $\left\langle X, R^{X}\right\rangle, X \subseteq V,|X|=2$, such that $\operatorname{umax}\left(R^{X, \mathcal{R}}\right)$ constitute a $(2,3)$-strategy for $\mathcal{P}^{\mathcal{R}}$. This collection exists by (S1) for $\mathcal{R}$. Let $R^{X, \mathcal{R}^{\prime}}$ denote the set of $\mathcal{R}^{\prime}$ compatible tuples from $R^{X, \mathcal{R}}$. It suffices to show that for any tuple $(a, b) \in$ $\operatorname{umax}\left(R^{X, \mathcal{R}^{\prime}}\right), X=\{x, y\}$, and any $w \notin\{x, y\}$ there is $c \in \mathbb{A}_{w}$ such that $(a, c) \in \operatorname{umax}\left(R^{\{x, w\}, \mathcal{R}^{\prime}}\right),(b, c) \in \operatorname{umax}\left(R^{\{y, w\}, \mathcal{R}^{\prime}}\right)$; then the proof can be completed by (S3) for $\mathcal{R}^{\prime}$ (to be proved later). By (S1) for $\mathcal{R}$ there is $d \in \mathbb{A}_{w}$ such that $(a, d) \in \operatorname{umax}\left(R^{\{x, w\}, \mathcal{R}}\right),(b, d) \in \operatorname{umax}\left(R^{\{y, w\}, \mathcal{R}}\right)$.

Consider the relation $R$ given by

$$
R(x, y, w)=R^{\{x, y\}}(x, y) \wedge R^{\{x, w\}}(x, w) \wedge R^{\{y, w\}}(y, w),
$$

and let $R^{\mathcal{R}}, R^{\mathcal{R}^{\prime}}$ be the set of $\mathcal{R}$ - and $\mathcal{R}^{\prime}$-compatible tuples from $R$, respectively. As is easily seen, element $c \in \mathbb{A}_{w}$ satisfies the required conditions if and only if $(a, b, c) \in R^{\mathcal{R}^{\prime}}$ and $(a, c) \in \operatorname{umax}\left(\operatorname{pr}_{x w} R^{\mathcal{R}^{\prime}}\right),(b, c) \in \operatorname{umax}\left(\operatorname{pr}_{y w} R^{\mathcal{R}^{\prime}}\right)$.

By (S4) $R^{\mathcal{R}}$ is a subalgebra of $R$; moreover, by (S1) the binary projections of $R^{\mathcal{R}}$ contain umax $\left(R^{\{x, y\}, \mathcal{R}}\right)$, umax $\left(R^{\{x, w\}, \mathcal{R}}\right)$, umax $\left(R^{\{y, w\}, \mathcal{R}}\right)$, respectively. Also, by Lemmas 612) and 77 $R^{\mathcal{R}}, R^{\mathcal{R}^{\prime}}$ are polynomially closed in $R$. We consider a number of cases. 
CASE 1. $w \notin W$.

If, say, $x \notin W$ then $R^{\{x, w\}, \mathcal{R}}=R^{\{x, w\}, \mathcal{R}^{\prime}}$. If $x \in W$, then by construction and the Congruence Lemma 64 either $\operatorname{umax}\left(R^{\{x, w\}, \mathcal{R}}\right)=\operatorname{umax}\left(R^{\{x, w\}, \mathcal{R}^{\prime}}\right)$ if $\tau_{x}$ is the full congruence, or $\operatorname{umax}\left(R^{\{x, w\}, \mathcal{R}} \cap\left(B_{x}^{\prime} \times B_{w}\right)\right) \subseteq \operatorname{umax}\left(R^{\{x, w\}, \mathcal{R}^{\prime}}\right)$ otherwise. In either case $(a, d) \in R^{\{x, w\}, \mathcal{R}^{\prime}}$. Similarly, $(b, d) \in R^{\{y, w\}, \mathcal{R}^{\prime}}$. Therefore $(a, b, d) \in R^{\mathcal{R}^{\prime}}$, and by the Maximality Lemma 27(5) there is always $c$ such that $(a, c) \in \operatorname{umax}\left(R^{\{x, w\}, \mathcal{R}^{\prime}}\right),(b, c) \in \operatorname{umax}\left(R^{\{y, w\}, \mathcal{R}^{\prime}}\right)$.

Therefore we may assume that $w \in W$.

If $x \in W$ (or $y \in W$, or $x, y \in W$ ) then let $\tau_{x}, \tau_{x w}$ (respectively, $\tau_{y}, \tau_{y w}$, or $\tau_{x y}$ ) be as in Lemma 73. If $x \notin W$ (or $y \notin W$ ) then let $\tau_{x}=\beta_{x}, \tau_{x w}=\tau_{w}$ (more precisely, $\tau_{x w}=\beta_{x} \times \tau_{w}$ ), and $\tau_{x y}=\tau_{y}$ (respectively, $\tau_{y}=\beta_{y}, \tau_{y w}=\tau_{w}$ ). We view all these congruences interchangeably: as congruences of $R$ in the natural way and their restrictions to $\mathcal{R}$-compatible tuples as congruences of $R^{\mathcal{R}}$, or as congruences of the corresponding projections of $R$ and $R^{\mathcal{R}}$. By Lemma 73 if $\tau^{\prime}$ is one of these congruences, say, $\tau^{\prime}=\tau_{X}$ for $X \subseteq\{x, y, w\}$, then $\tau^{\prime}$ viewed as a congruence of $R^{X}$ is either the full congruence on $R^{X, \mathcal{R}}$ or $\tau^{\prime} \prec \bar{\beta}_{X}$. Therefore $\tau^{\prime}$ viewed as a congruence of $R$ is either the full congruence on $R^{\mathcal{R}}$, or $\tau^{\prime} \prec$ $\left(\beta_{x} \times \beta_{y} \times \beta_{w}\right)$ in $\operatorname{Con}(R)$; in the latter case we will say that $\tau^{\prime}$ is maximal. Also let $\tau=\tau_{x y} \wedge \tau_{x w} \wedge \tau_{y w}$. Again by Lemma $73 R^{\mathcal{R}} / \tau$ is a module.

Let $R_{a}^{*}, R_{b}^{*}$ be the sets of tuples $\mathbf{a} \in R^{\mathcal{R}}$ satisfying $\mathbf{a}[x]=a, \mathbf{a}[y]=b$, respectively; note that $(a, b, d) \in R_{x}^{*} \cap R_{y}^{*}$. We consider several cases of what the congruences introduced earlier can be.

CASE 2. $\tau_{x}$ is the full congruence and $\tau_{x w}=\tau_{w}$, or $\tau_{y}$ is the full congruence and $\tau_{y w}=\tau_{w}$.

Suppose $\tau_{x}$ is the full congruence, the other option is similar.

SUBCASE $2.1 \tau_{y w}$ is the full congruence.

As $\tau_{y w} \leq \tau_{w}$, if $\tau_{y w}$ is a full congruence, $\tau_{w}=\tau_{x w}$ is also the full congruence. Therefore, $(a, d) \in R^{\{x, w\}, \mathcal{R}^{\prime}},(b, d) \in R^{\{y, w\}, \mathcal{R}^{\prime}}$, and we are done.

SUBCASE $2.2 \tau_{y}$ is maximal while $\tau_{w}$ is full.

Since any tuple $(b, x) \in R^{\{y, w\}, \mathcal{R}}$ also belongs to $R^{\{y, w\}, \mathcal{R}^{\prime}}$ in this case, again $(a, d) \in R^{\{x, w\}, \mathcal{R}^{\prime}},(b, d) \in R^{\{y, w\}, \mathcal{R}^{\prime}}$

SUBCASE 2.3 Both $\tau_{y}$ and $\tau_{w}$ are maximal.

In this case, as $\tau_{y w} \leq \tau_{y} \wedge \tau_{w}$ and all three congruences are maximal, we have $\tau_{y w}=\tau_{y}=\tau_{w}$. Also, $\tau_{w}$ (viewed as a congruence of $\operatorname{pr}_{w} R^{\mathcal{R}}$ ) is the link congruence with respect to $\operatorname{pr}_{y w} R^{\mathcal{R}}$, and so $(b, d) \in R^{\{y, w\}, \mathcal{R}^{\prime}}$. This also implies, since $(a, b) \in \operatorname{pr}_{x y} R^{\mathcal{R}}$, that $(a, d) \in R^{\{x, w\}, \mathcal{R}^{\prime}}$. 
SUBCASE $2.4 \tau_{w}$ is maximal while $\tau_{y}$ is full.

Let $B_{z}^{*}=\operatorname{pr}_{z} R^{\mathcal{R}}$ for $z \in\{x, y, w\}$. By the Congruence Lemma 64 umax $\left(B_{x}^{*}\right) \times$ $B_{w}^{*} / \tau_{w} \subseteq \operatorname{pr}_{x w} R^{\mathcal{R}}$ and $\operatorname{umax}\left(B_{y}^{*}\right) \times B_{w}^{*} / \tau_{w} \subseteq \operatorname{pr}_{y w} R^{\mathcal{R}}$. Indeed, otherwise $\operatorname{pr}_{x w} R^{\mathcal{R}} / \tau_{w}$ would be the graph of a mapping $\nu: B_{x}^{*} \rightarrow B_{w}^{*} / \tau_{w}$ contradicting the assumption that $\tau_{x}$ is the full congruence; similar for $B_{y}^{*}$. Therefore $R_{a}^{*} / \tau_{w}=$ $R_{b}^{*} / \tau_{w}=R^{\mathcal{R}} / \tau_{w}$ and since $(a, b)$ is u-maximal in a $\tau_{x y}$-block, $d$ can be chosen such that $(a, b, d)$ is u-maximal in a $\tau_{w}$-block. Then by Lemma79 $\left(R_{a}^{*} \cap R_{b}^{*}\right) / \tau_{w}=$ $R^{\mathcal{R}} / \tau_{w}$ and the result follows.

SUBCASE 2.5. $\tau_{y w} \prec \tau_{y}=\tau_{w}$ and $\tau_{y}, \tau_{w}$ are full congruences.

Again by the Congruence Lemma $64 \operatorname{umax}\left(B_{x}^{*}\right) \times \mathrm{pr}_{y w} R^{\mathcal{R}} / \tau_{y w} \subseteq R^{\mathcal{R}}$ and therefore $R_{a}^{*} / \tau_{y w}=R^{\mathcal{R}} / \tau_{y w}$. Also, let $Q$ be the union of all $\tau_{y w}$-blocks of $R^{\mathcal{R}}$ whose intersection with $R_{b}^{*}$ is nonempty, this is clearly a subalgebra. Also, let $R_{a}^{* *}=R_{a}^{*} \cap$ $Q$. Note that, since $\tau_{y}$ is the full congruence, and therefore $\operatorname{umax}\left(B_{y}^{*}\right) \subseteq R^{\{y, w\}, \mathcal{R}^{\prime}}$, we have $R^{\{y, w\}, \mathcal{R}^{\prime}} \cap \operatorname{pr}_{y w} Q \neq \varnothing$. Then $R_{a}^{* *} / \tau_{y w}=Q / \tau_{y w}$ and $R_{b}^{*} / \tau_{y w}=Q / \tau_{y w}$. Then as before the result follows by Lemma 79 .

CASE 3. $\tau_{x}, \tau_{y}$ are maximal congruences.

In this case $\tau_{x w}=\tau_{x}, \tau_{y w}=\tau_{y}$, and depending on whether or not $\tau_{w}$ is maximal we proceed as in Cases 2.2 or 2.3.

If $\tau_{w}$ is a maximal congruence or $\tau_{x w}$ or $\tau_{y w}$ is the full congruence, the result follows from one of the previous cases. Therefore the only remaining case is

CASE 4. $\tau_{x w}$ and $\tau_{y w}$ are maximal, while $\tau_{x}, \tau_{y}, \tau_{w}$ are not.

Let again $B_{z}^{*}=\operatorname{pr}_{z} R^{\mathcal{R}}$ for $z \in\{x, y, w\}$. If $(v, \alpha, \beta) \notin \mathcal{W}^{\prime}$ or $w \notin \operatorname{MAX}(\mathcal{P})$, that is, $\mu_{w}^{Y}=\underline{0}_{w}$ for any choice of $Y$ in (R1),(R2), then the required $c \in B_{w}^{*}$ exists, since $(a, b)$ can be extended to a solution from $\mathcal{S}_{W}^{\mathcal{R}^{\prime}}$ or $\mathcal{S}_{W, Y}^{\mathcal{R}^{\prime}}, Y=\operatorname{MAX}(\mathcal{P})-$ $\{x, y\}$ by construction.

Suppose that $(v, \alpha, \beta) \in \mathcal{W}^{\prime}$ and $\mu_{w}^{Y}=\mu_{w}$ for $Y=\operatorname{MAX}(\mathcal{P})-\{x, y\}$. Let $Q_{x}$ be a subalgebra of the product $\mathbb{A}_{x} \times \mathbb{A}_{w} \times \mathbb{A}_{v} / \alpha$ that consists of all triples $\left(a^{\prime}, b^{\prime}, c^{\prime}\right)$ such that there is a solution $\varphi \in \mathcal{S}_{W, Y}$ with $\varphi(x)=a^{\prime}, \varphi(w)=b^{\prime}$, and $\varphi(v) \in c^{\prime}$. Let $B_{v}^{*}=A_{\mathcal{R}, v}$. By block-minimality $Q_{x}$ is indeed a subdirect product and by (S2) for $\mathcal{R}$ we have umax $\left(R^{\{x, w\}, \mathcal{R}}\right) \subseteq \mathrm{pr}_{x w}\left(Q_{x} \cap\left(B_{x}^{*} \times B_{w}^{*} \times B_{v}^{*} / \alpha\right)\right.$ and $\operatorname{pr}_{v}\left(Q_{x} \cap\left(B_{x}^{*} \times B_{w}^{*} \times B_{v}^{*} / \alpha\right)=B^{*} / \alpha\right.$. Also, by Lemma 61 (3) $Q_{x}$ is polynomially closed. Relation $Q_{y}$ is defined in a similar way. Let also

$$
Q(x, y, w, v)=Q_{x}(x, w, v) \wedge Q_{y}(y, w, v),
$$

and $Q^{\prime}=Q \cap \bar{B}$. Let $Q^{a}=\left\{\mathbf{a} \in Q^{\prime} \mid \mathbf{a}[x]=a\right\}, Q^{b}=\left\{\mathbf{a} \in Q^{\prime} \mid \mathbf{a}[y]=b\right\}$, and $\alpha^{\prime}=\beta_{x} \times \beta_{y} \times \beta_{w} \times \alpha$. By the assumption that $\tau_{x}, \tau_{y}$ are full congruences $Q^{a} / \alpha^{\prime}=Q^{b} / \alpha^{\prime}=Q^{\prime} / \alpha^{\prime}$. Therefore, if we prove that $(a, b) \in \operatorname{pr}_{x y} Q^{\prime}$, we obtain 
the result by Lemma79. Note that if this is the case, since $(a, b) \in \operatorname{umax}\left(R^{\{x, y\}, \mathcal{R}}\right.$, there is also $\left(a, b, c^{\prime}, d^{\prime}\right) \in Q^{\prime}$ that is u-maximal in an $\alpha^{\prime}$-block.

To this end consider the relations

$$
S\left(x, y, w, v, v^{\prime}\right)=Q_{x}(x, w, v) \wedge Q_{y}\left(y, w, v^{\prime}\right),
$$

and $S^{\prime}=S \cap \bar{B}$. In a similar way we define $S^{a}=\left\{\mathbf{a} \in S^{\prime} \mid \mathbf{a}[x]=a\right\}$, $S^{b}=\left\{\mathbf{a} \in S^{\prime} \mid \mathbf{a}[y]=b\right\}$. By (S1) there are $d \in B_{w}^{*}$ and $e^{\prime}, e^{\prime \prime} \in B_{v}^{*} / \alpha$ such that $\left(a, b, d, e^{\prime}, e^{\prime \prime}\right) \in S^{\prime}$. Also by construction (R2) there are $a^{\prime} \in B_{x}^{*}, b^{\prime} \in B_{y}^{*}$, $d_{1}, d_{2} \in B_{w}^{*}, d_{1} \stackrel{\mu_{w}}{=} d_{2}$ and $e \in B_{v}^{*}$, such that $\left(a, b^{\prime}, d_{1}, e, e\right),\left(a^{\prime}, b, d_{2}, e, e\right) \in S^{\prime}$. Recall that $B_{v}^{*} / \alpha$ is a module. Let $\delta$ be the skew congruence of $B_{v}^{*} / \alpha \times B_{v}^{*} / \alpha$, that is, a congruence such that $\Delta=\left\{\left(c_{1}, c_{2}\right), \mid c_{1} \stackrel{\alpha}{\equiv} c_{2}\right\}$ is a $\delta$-block. Let $\delta^{\prime}$ be the congruence of $S^{\prime}$ given by $\mathbf{c} \stackrel{\delta^{\prime}}{\equiv} \mathbf{d}$ if and only if $\mathrm{pr}_{v v^{\prime}} \mathbf{c} \stackrel{\delta}{\equiv} \operatorname{pr}_{v v^{\prime}} \mathbf{d}$. Note that $\delta^{\prime}<\bar{\beta}$ in $\operatorname{Con}(S)$. Let $\Delta^{\prime}$ be the $\delta^{\prime}$-block corresponding to $\Delta$, that is, $\Delta=\operatorname{pr}_{v v^{\prime}} \Delta^{\prime}$. Since $S^{a} \cap S^{b} \neq \varnothing$ and $\left(a, b, d, e^{\prime}, e^{\prime \prime}\right)$ can be chosen to be u-maximal in a $\delta^{\prime}$-block, and $S^{a} \cap \Delta^{\prime} \neq \varnothing, S^{b} \cap \Delta^{\prime} \neq \varnothing$, by Lemma79 we have $S^{a} \cap S^{b} \cap \Delta^{\prime} \neq \varnothing$. The result follows.

\subsubsection{Conditions (S2), (S3)}

In this section we prove that $\mathcal{R}^{\prime}$ satisfies conditions (S2) and (S3). We first prove (S2) and then show what in the proof has to be changed to obtain a proof of (S3).

As before, let $W=W_{v, \alpha \beta}$.

Lemma $80 \mathcal{R}^{\prime}$ satisfies (S2). That is, for every $\left(w_{1}, \gamma_{1}, \delta_{1}\right),\left(w_{2}, \gamma_{2}, \delta_{2}\right) \in \mathcal{W}\left(\bar{\beta}^{\prime}\right)$ $\left(\right.$ let $\left.W_{1}=W_{w_{1}, \gamma_{1} \delta_{1}}, W_{2}=W_{w_{2}, \gamma_{2} \delta_{2}}\right)$ and every $\mathbf{a} \in \operatorname{umax}\left(\operatorname{pr}_{\mathbf{s} \cap W_{1} \cap W_{2}} R_{C, w_{1}, \gamma_{1} \delta_{1}}^{\prime}\right)$ it holds: if $\left(w_{2}, \gamma_{2}, \delta_{2}\right) \notin \mathcal{W}^{\prime}$ then a extends to an $\mathcal{R}^{\prime}$-compatible solution $\varphi$ of $\mathcal{P}_{W_{2}}$; otherwise if $\left(w_{1}, \gamma_{1}, \delta_{1}\right) \notin \mathcal{W}^{\prime}$ then a extends to an $\mathcal{R}^{\prime}$-compatible solution of $\mathcal{P}_{W_{2}} / \bar{\mu}^{Y_{1}}$, where $Y_{1}=\operatorname{MAX}(\mathcal{P})-\left(W_{1} \cap W_{2}\right)$; and if $\left(w_{1}, \gamma_{1}, \delta_{1}\right) \in \mathcal{W}^{\prime}$ then $\mathbf{a}$ extends to an $\mathcal{R}^{\prime}$-compatible solution of $\mathcal{P}_{W_{2}} / \bar{\mu}^{Y_{2}}$, where $Y_{2}=\operatorname{MAX}(\mathcal{P})-\mathbf{s}$.

Take $C=\langle\mathbf{s}, R\rangle$ and $\left(w_{1}, \gamma_{1}, \delta_{1}\right),\left(w_{2}, \gamma_{2}, \delta_{2}\right) \in \mathcal{W}\left(\bar{\beta}^{\prime}\right)$ and let $W_{1}=W_{w_{1}, \gamma_{1} \delta_{1}}$, $W_{2}=W_{w_{2}, \gamma_{2} \delta_{2}}$, and $U=\mathbf{s} \cap W_{1} \cap W_{2}$. Let $\mathbf{a} \in \operatorname{umax}\left(\operatorname{pr}_{U} R_{C, w_{1}, \gamma_{1} \delta_{1}}^{\prime}\right)$. Depending on whether or not $\left(w_{1}, \gamma_{1}, \delta_{1}\right),\left(w_{2}, \gamma_{2}, \delta_{2}\right) \in \mathcal{W}^{\prime}$ we need to show that a can be extended to a solution of $\mathcal{P}_{W_{2}} / \bar{\mu}^{Y}$, where $Y$ is either empty, if $\left(w_{2}, \gamma_{2}, \delta_{2}\right) \notin$ $\mathcal{W}^{\prime}$, or $Y=\operatorname{MAX}(\mathcal{P})-W_{1}$ if $\left(w_{1}, \gamma_{1}, \delta_{1}\right) \notin \mathcal{W}^{\prime}$ and $\left(w_{2}, \gamma_{2}, \delta_{2}\right) \in \mathcal{W}^{\prime}$, and $Y=\operatorname{MAX}(\mathcal{P})-\mathbf{s}$ if $\left(w_{1}, \gamma_{1}, \delta_{1}\right),\left(w_{2}, \gamma_{2}, \delta_{2}\right) \in \mathcal{W}^{\prime}$. The three cases are quite similar so we will unify the proofs as much as possible.

Let $\mathcal{P}_{W^{\prime}}^{\prime}$ denote the problem $\mathcal{P}_{W^{\prime}} / \bar{\mu}^{Y}$ for a set $W^{\prime} \subseteq V$ and $\mathcal{S}_{W^{\prime}}^{\prime}$ denote its set of solutions. Then we need to show that $\mathbf{a} \in \operatorname{pr}_{U} \mathcal{S}_{W_{2}}^{\prime \mathcal{R}^{\prime}}$. 
First, we will show that there are $\mathcal{R}^{\prime}$-compatible solutions of $\mathcal{P}_{W \cap W_{2}}^{\prime}$. This statement would be trivial, as $\mathcal{S}_{W \cap W_{2}}^{\mathcal{R}^{\prime}}$ contains $\operatorname{pr}_{W \cap W_{2}} \mathcal{S}_{W}^{\mathcal{R}^{\prime}}$, if $Y$ was always empty. Otherwise a simple proof is needed. Note that this is the only statement where we consider the three options for $Y$ separately. Next, we identify the properties of $\mathcal{P}_{W^{\prime}} / \bar{\mu}^{Y}$ required for proving (S2) and show that the problem satisfies them for all choices of $Y$. The rest of the proof will be given only using these properties; thus we use the same argument in all the three cases of $Y$.

If $\tau_{C^{\prime}, w_{2}, \gamma_{2} \delta_{2}}$ is the full congruence of $R_{C^{\prime}, w_{2}, \gamma_{2} \delta_{2}} / \bar{\mu}^{Y}$ for all $C^{\prime} \in \mathcal{C}$, then $\operatorname{umax}\left(\mathcal{S}_{W_{2}}^{\prime \mathcal{R}}\right)=\operatorname{umax}\left(\mathcal{S}_{W_{2}}^{\prime \mathcal{R}^{\prime}}\right)$ and there is nothing to prove. Thus we will always assume that there is $C^{\prime} \in \mathcal{C}$ such that $\tau_{C^{\prime}, w_{2}, \gamma_{2} \delta_{2}}$ is maximal on $R_{C^{\prime}, w_{2}, \gamma_{2} \delta_{2}} / \bar{\mu}^{Y}$.

Lemma 81 The relations $\mathcal{S}_{W \cap W_{2}}^{\prime \mathcal{R}}$ and $\mathcal{S}_{W \cap W_{2}}^{\prime \mathcal{R}^{\prime}}$ are nonempty.

Proof: The set $\mathcal{S}_{W \cap W_{2}}^{\prime \mathcal{R}}$ is nonempty, as it contains $\mathrm{pr}_{W \cap W_{2}} \mathcal{S}_{W_{2}}^{\prime \mathcal{R}}$, which is nonempty by (S2) for $\mathcal{R}$. If $(v, \alpha, \beta) \notin \mathcal{W}^{\prime}$, then $\mathcal{S}_{W \cap W_{2}}^{\prime \mathcal{R}^{\prime}}$ contains $\operatorname{pr}_{W \cap W_{2}} \mathcal{S}_{W}^{\prime \mathcal{R}^{\prime}}$ or its factor modulo $\bar{\mu}^{Y}$ (if $Y \neq \varnothing$ ), which is nonempty. So, let $(v, \alpha, \beta) \in \mathcal{W}(\bar{\beta})$.

Suppose $\left(w_{2}, \gamma_{2}, \delta_{2}\right) \notin \mathcal{W}^{\prime}$ and so $Y=\varnothing$, and $C_{1}=\left\langle\mathbf{s}_{1}, R_{1}\right\rangle \in \mathcal{C}$ is a constraint such that $\tau_{C_{1}, w_{2}, \gamma_{2} \delta_{2}}$ is nontrivial on $R_{C_{1}, w_{2}, \gamma_{2} \delta_{2}}$ and $\mathbf{b} \in \operatorname{umax}\left(R_{C_{1}, w_{2}, \gamma_{2} \delta_{2}}^{\prime}\right)$. By (S2) for $\mathcal{R}$ tuple $\mathbf{b}$ can be extended to a solution $\varphi$ of $\mathcal{P}_{W} / \bar{\mu}^{Y_{1}}, Y_{1}=\operatorname{MAX}(\mathcal{P})-$ $W_{2}$. Then $\varphi(v) \in B_{v}^{\prime} / \mu_{v}^{Y_{1}}$ and therefore for any $C_{2}=\left\langle\mathbf{s}_{2}, R_{2}\right\rangle \in \mathcal{C}$ and any $(u, \eta, \theta) \in \mathcal{W}(\bar{\beta})$ we have $\varphi\left(\mathbf{s}_{2} \cap W \cap W_{u, \eta \theta}\right) \in \operatorname{pr}_{\mathbf{s} \cap W \cap W_{u, \eta \theta}} R_{C_{2}, u, \eta \theta}^{\prime} / \bar{\mu}^{Y_{1}}$, that is, $\varphi\left(W \cap W_{2}\right) \in \mathcal{S}_{W \cap W_{2}}^{\prime \mathcal{R}^{\prime}} / \bar{\mu}^{Y_{1}}=\mathcal{S}_{W \cap W_{2}}^{\prime \mathcal{R}^{\prime}}$.

Suppose now that $(v, \alpha, \beta),\left(w_{2}, \gamma_{2}, \delta_{2}\right) \in \mathcal{W}^{\prime}$. If $\left(w_{1}, \gamma_{1}, \delta_{1}\right) \notin \mathcal{W}^{\prime}$ and $Y=$ $\operatorname{MAX}(\mathcal{P})-W_{1}$, then we apply the argument above to the problem $\mathcal{P}_{W} / \bar{\mu}^{\operatorname{MAX}(\mathcal{P})-W_{1}}$. If $\left(w_{1}, \gamma_{1}, \delta_{1}\right) \in \mathcal{W}^{\prime}$ and $Y=\operatorname{MAX}(\mathcal{P})-\mathbf{s}$, then we consider the problem $\mathcal{P}_{W} / \bar{\mu}^{\operatorname{MAX}(\mathcal{P})-\mathrm{s}}$.

For any possible choice of $Y$ the sets $\mathcal{S}_{W_{2}}^{\prime}, \mathcal{S}_{W_{2}}^{\prime \mathcal{R}}, \mathcal{S}_{W_{2}}^{\prime \mathcal{R}^{\prime}}$ satisfy the following conditions. For the rest of the proof we only need these conditions, and therfore the argument is valid for all choices of $Y$. First of all if $Y \neq \varnothing$ the set $W$ may need to be redefined. By the definition of $W$ for every $w \in W$ there are $\gamma_{w}, \delta_{w} \in \operatorname{Con}\left(\mathbb{A}_{w}\right)$ such that $\gamma_{w} \prec \delta_{w} \leq \beta_{w}$ and $\left(\alpha, \beta_{v}\right),\left(\gamma_{w}, \delta_{w}\right)$ cannot be separated in $\mathcal{P}$. If $w \in Y$ and $\gamma_{w}=\underline{0}_{w}, \delta_{w}=\mu_{w}$ is the only choice for $\gamma_{w}, \delta_{w}$, variable $w$ is removed from $W$. Set $W_{2}$ does not have to be modified. The conditions needed are:

(X1) $\mathcal{P}_{W_{2}, Y}$ is minimal by block-minimality.

(X2) For every $(u, \eta, \theta) \in \mathcal{W}(\bar{\beta})$ and $C^{\prime}=\left\langle\mathbf{s}^{\prime}, R^{\prime}\right\rangle$ any tuple from umax $\left(\operatorname{pr}_{Z} R_{C^{\prime}, u, \eta \theta}\right) / \bar{\mu}^{Y}$, where $Z=W_{u, \eta \theta} \cap W_{2} \cap \mathbf{s}^{\prime}$, can be extended to a solution from $\mathcal{S}_{W_{2}}^{\prime \mathcal{R}}$; by (S2). 
(X3) For every $(u, \eta, \theta) \in \mathcal{W}(\bar{\beta})$ and $C^{\prime}=\left\langle\mathbf{s}^{\prime}, R^{\prime}\right\rangle$ let $W^{\prime}=W_{u, \eta \theta} \cap W \cap W_{2} \cap \mathbf{s}^{\prime}$ and $\tau_{C^{\prime}}^{\prime}(u, \eta \theta)$ denote the restriction of $\tau_{C^{\prime}}$ on $R_{C^{\prime}, u, \eta \theta}^{*}=\operatorname{pr}_{W^{\prime}} R_{C^{\prime}, u, \eta \theta} / \bar{\mu}^{Y}$, that is, $(\mathbf{b}, \mathbf{c}) \in \tau_{C^{\prime}}^{\prime}(u, \eta \theta)$ if there are $\mathbf{b}^{\prime}, \mathbf{c}^{\prime} \in R_{C^{\prime}, v, \alpha \beta_{v}} / \bar{\mu}^{Y}$ such that $\operatorname{pr}_{W^{\prime}} \mathbf{b}^{\prime}=\mathbf{b}, \operatorname{pr}_{W^{\prime}} \mathbf{c}^{\prime}=\mathbf{c}$, and $\left(\mathbf{b}^{\prime}, \mathbf{c}^{\prime}\right) \in \tau_{C^{\prime}}$. By Lemma $73 \tau_{C^{\prime}}^{\prime}(u, \eta \theta)$ is either the full congruence on $R_{C^{\prime}, u, \eta \theta}^{*}$, or a maximal one. In the latter case $\operatorname{typ}\left(\tau_{C^{\prime}}^{\prime}(u, \eta \theta), \bar{\beta}_{W^{\prime}}\right)=\mathbf{2}$.

(X4) For every $w \in W \cap W_{2}$ there are $\gamma_{w}, \delta_{w} \in \operatorname{Con}\left(\mathbb{A}_{w} / \mu_{w}^{Y}\right)$ such that $\mu_{w}^{Y} \leq$ $\gamma_{w} \prec \delta_{w} \leq \beta_{w}$ and $\left(\alpha, \beta_{v}\right),\left(\gamma_{w}, \delta_{w}\right)$ cannot be separated in $\mathcal{P}$.

(X5) For every $(u, \eta, \theta) \in \mathcal{W}(\bar{\beta}), C^{\prime}=\left\langle\mathbf{s}^{\prime}, R^{\prime}\right\rangle$, and any $w \in W^{\prime}=W_{u, \eta \theta} \cap$ $W \cap W_{2} \cap \mathbf{s}^{\prime}$, if $\tau_{C^{\prime}}^{\prime}(u, \eta \theta)$ is maximal, then for every polynomial $f$ of $\mathcal{S}_{W_{2}}^{\prime}$ we have $f\left(\delta_{w}\right) \subseteq \gamma_{w}$ if and only if $f\left(\bar{\beta}_{W^{\prime}}\right) \subseteq \tau_{C^{\prime}}^{\prime}(u, \eta \theta)$; by Lemma74

We are now in a position to prove Lemma 80 .

Proof: (of Lemma 80) Recall that we need to prove that any $\mathbf{a} \in \operatorname{umax}\left(\operatorname{pr}_{U} R_{C, w_{1}, \gamma_{1} \delta_{1}}^{\prime}\right)$ can be extended to a solution from $\mathcal{S}_{W_{2}}^{\prime \mathcal{R}^{\prime}}$. By (S2) a can be extended to a tuple $\mathbf{b} \in \mathcal{S}_{W_{2}}^{\prime \mathcal{R}}$. We will prove that a required extesion $\mathbf{c} \in \mathcal{S}_{W_{2}}^{\prime \mathcal{R}^{\prime}}$ can be found such that $\operatorname{pr}_{W_{2}-W} \mathbf{c}=\operatorname{pr}_{W_{2}-W} \mathbf{b}$ (if $\mathbf{b}$ is chosen well). Therefore, we will need to study the relationship of tuples from $\operatorname{pr}_{W_{2} \cap W} \mathcal{S}_{W_{2}}^{\prime \mathcal{R}}$ extending a and the tuples from $\operatorname{pr}_{W_{2}-W} \mathcal{S}_{W_{2}}^{\prime \mathcal{R}}$. This is done in Claim 1. Since tuples from $\operatorname{pr}_{W_{2} \cap W} \mathcal{S}_{W_{2}}^{\prime \mathcal{R}}$ are only considered modulo congruences of the form $\tau_{C^{\prime}}$, while a is considered without factoring, the main technical hurdle is to 'disentangle' a from the quotient of $\operatorname{pr}_{W_{2} \cap W} \mathcal{S}_{W_{2}}^{\prime \mathcal{R}}$. To this end we consider a congruence $\tau$ on $\mathcal{S}_{W_{2} \cap W}^{\prime \mathcal{R}}$ that isolates $\mathcal{R}^{\prime}$ compatible tuples from there, and then introduce two auxiliary relations $Q$ and $S$.

We would like to define a congruence similar to $\tau_{C}$ on $\mathcal{S}_{W \cap W_{2}}^{\prime}$. It cannot be done in the same straightforward way, since $\mathcal{S}_{W \cap W_{2}}^{\prime} \neq \operatorname{pr}_{W \cap W_{2}} \mathcal{S}_{W}^{\prime}$, so we define it as follows. For $C^{\prime}=\left\langle\mathbf{s}^{\prime}, R^{\prime}\right\rangle$ and $(u, \eta, \theta) \in \mathcal{W}(\bar{\beta})$ we extend the congruences $\tau_{C^{\prime}}^{\prime}(u, \eta \theta)$ to congruences of $\mathcal{S}_{W \cap W_{2}}^{\prime}$ using $\tau_{C^{\prime}}^{\prime}(u, \eta \theta) \times \prod_{x \in\left(W \cap W_{2}\right)-W^{\prime}} \underline{1}_{x}$, where $W^{\prime}=\mathbf{s}^{\prime} \cap W_{u, \eta \theta} \cap W \cap W_{2}$. Then the set $\mathcal{S}_{W \cap W_{2}}^{\prime \mathcal{R}^{\prime}}$ of $\mathcal{R}^{\prime}$-compatible tuples from $\mathcal{S}_{W \cap W_{2}}^{\prime \mathcal{R}}$ is a block of

$$
\tau=\bigwedge_{C^{\prime} \in \mathcal{C},(u, \eta, \theta) \in \mathcal{W}(\bar{\beta})} \tau_{C^{\prime}}^{\prime}(u, \eta \theta)
$$

let it be denoted by $\mathcal{S}^{*}$. Note that $\tau$ is a congruence of $\mathcal{S}_{W \cap W_{2}}^{\prime}$, but the interval $\left(\tau, \bar{\beta}_{W \cap W_{2}}\right)$ is not necessarily prime. By the observation above and Lemma 29 $\mathcal{S}_{W \cap W_{2}}^{\prime \mathcal{R}} /{ }_{\tau}$ is term equivalent to a module. We need to prove that there is c $\in \mathcal{S}_{W_{2}}^{\prime \mathcal{R}}$ such that $\varphi(U)=\mathbf{a}$ and $\varphi\left(W \cap W_{2}\right) \in \mathcal{S}^{*}$. In fact we prove a stronger statement, namely, that for any u-maximal tuple $\mathbf{b}$ from $\operatorname{pr}_{W_{2}-W} \mathcal{S}_{W_{2}}^{\prime \mathcal{R}}$ there is a solution $\varphi$ 
such that $\varphi(U)=\mathbf{a}, \varphi\left(W \cap W_{2}\right) \in \mathcal{S}^{*}$ and also $\varphi\left(W_{2}-W\right)=\mathbf{b}$. However, to formulate it precisely we need two additional constructions.

We need to describe the $\tau$-blocks that contain tuples extending a. In order to do that we separate the variables of a from the rest of $W_{2} \cap W$ by making an extra copy of them, as follows. Let $W \cap W_{2}=\left\{x_{1}, \ldots, x_{k}\right\}$ and $X=W \cap U=\left\{x_{1}, \ldots, x_{\ell}\right\}$, and $X^{\prime}=\left\{y_{1}, \ldots, y_{\ell}\right\}$. Let

$Q\left(x_{1}, \ldots, x_{k}, y_{1}, \ldots, y_{\ell}\right)=\mathcal{S}_{W \cap W_{2}}^{\prime \mathcal{R}}\left(x_{1}, \ldots, x_{k}\right) \wedge \operatorname{pr}_{X} \mathcal{S}_{W \cap W_{2}}^{\prime \mathcal{R}}\left(y_{1}, \ldots, y_{\ell}\right) \wedge \bigwedge_{i=1}^{\ell}\left(x_{i}=y_{i}\right)$,

and its factor $Q^{\prime}=Q / \tau^{\prime}$, where $\tau^{\prime}=\tau \times \underline{0}_{\operatorname{pr}_{X^{\prime}} \mathcal{S}_{W \cap W_{2}}^{\prime R}}$. Let $\eta_{1}, \eta_{2}$ denote the link congruences of $\mathcal{S}_{W \cap W_{2}}^{\prime \mathcal{R}} / \tau$ and $\operatorname{pr}_{X^{\prime}} \mathcal{S}_{W \cap W_{2}}^{\prime \mathcal{R}}$ with respect to $Q^{\prime}$, and let $\eta^{\prime}$ denote the congruence of $\mathcal{S}_{W \cap W_{2}}^{\prime \mathcal{R}}$ such that $\mathbf{c} \stackrel{\eta^{\prime}}{=} \mathbf{d}$ if and only if $\mathbf{c}^{\tau} \stackrel{\eta_{1}}{=} \mathbf{d}^{\tau}$. Then, as is easily seen, since $\operatorname{pr}_{W \cap W_{2}} Q / \tau$ is a module, $(\mathbf{b}, \mathbf{c}) \in \eta^{\prime}$ if and only if there are $\mathbf{b}^{\prime}, \mathbf{c}^{\prime} \in \mathcal{S}_{W \cap W_{2}}^{\mathcal{R}}$ such that $\left(\mathbf{b}, \mathbf{b}^{\prime}\right),\left(\mathbf{c}, \mathbf{c}^{\prime}\right) \in \tau$ and $\operatorname{pr}_{X} \mathbf{b}^{\prime}=\operatorname{pr}_{X} \mathbf{c}^{\prime}$.

Now, we introduce a similar construction for $\mathcal{S}_{W_{2}}^{\prime}$. The goal is to compare the two. Let $W_{2}=\left\{x_{1}, \ldots, x_{m}\right\}$ (recall that $W \cap W_{2}=\left\{x_{1}, \ldots, x_{k}\right\}$ ) and define a relation $S\left(x_{1}, \ldots, x_{m}, y_{1}, \ldots, y_{\ell}\right)$ as follows:

$S\left(x_{1}, \ldots, x_{m}, y_{1}, \ldots, y_{\ell}\right)=\mathcal{S}_{W_{2}}^{\prime \mathcal{R}}\left(x_{1}, \ldots, x_{m}\right) \wedge \operatorname{pr}_{X} \mathcal{S}_{W_{2}}^{\prime \mathcal{R}}\left(y_{1}, \ldots, y_{\ell}\right) \wedge \bigwedge_{i=1}^{\ell}\left(x_{i}=y_{i}\right)$,

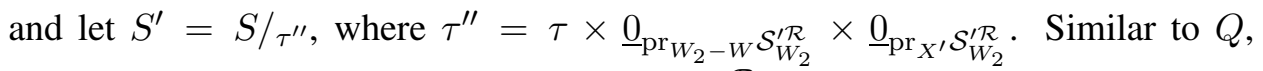
let $\theta_{1}, \theta_{2}$ be the link congruences of $\operatorname{pr}_{W \cap W_{2}} \mathcal{S}_{W_{2}}^{\prime \mathcal{R}} / \tau$ and $\operatorname{pr}_{H} S$, where $H=X^{\prime} \cup$ $\left\{x_{k+1}, \ldots, x_{m}\right\}$, with respect to $S^{\prime}$, and let $\theta^{\prime}$ denote the congruence of $\operatorname{pr}_{W \cap W_{2}} \mathcal{S}_{W_{2}}^{\mathcal{R}}$ given by $\mathbf{c} \stackrel{\theta^{\prime}}{\equiv} \mathbf{d}$ if and only if $\mathbf{c}^{\tau} \stackrel{\theta_{1}}{\equiv} \mathbf{d}^{\tau}$. Then immediately by the definition $(\mathbf{b}, \mathbf{c}) \in \theta^{\prime}$ if and only if there are $\mathbf{b}^{\prime}, \mathbf{c}^{\prime} \in \operatorname{pr}_{W \cap W_{2}} \mathcal{S}_{W_{2}}^{\prime \mathcal{R}}$ and $\mathbf{d} \in \operatorname{pr}_{H} S$ such that $\left(\mathbf{b}, \mathbf{b}^{\prime}\right),\left(\mathbf{c}, \mathbf{c}^{\prime}\right) \in \tau, \operatorname{pr}_{X} \mathbf{b}^{\prime}=\operatorname{pr}_{X} \mathbf{c}^{\prime}=\operatorname{pr}_{X} \mathbf{d}$, and $\left(\operatorname{pr}_{\left(W \cap W_{2}\right)-X} \mathbf{b}^{\prime}, \mathbf{d}\right)$, $\left(\operatorname{pr}_{\left(W \cap W_{2}\right)-X} \mathbf{c}^{\prime}, \mathbf{d}\right) \in \mathcal{S}_{W_{2}}^{\prime \mathcal{R}}$.

We are interested in congruences $\eta_{1}$ and $\theta_{1}$. The first of them indicates which $\tau$-blocks extensions of $\operatorname{pr}_{X}$ a can belong to. The second congruence also indicates to which $\tau$-blocks extensions of a to a solution from $\mathcal{S}_{W_{2}}^{\prime \mathcal{R}}$ can belong to. Clearly, $\theta_{1} \subseteq \eta_{1}$. We prove however, that in both cases the set of attainable $\tau$-blocks is the same. This essentially means that if a $\tau$-block can be extended to a solution from $\mathcal{S}_{W_{2}}^{\mathcal{R}}$, it can be extended in an almost arbitrary way.

CLAIM 1. (1) $S^{\prime \prime}=\left(\mathrm{pr}_{W \cap W_{2}} S\right) / \tau$ is a union of $\eta_{1}$-blocks;

(2) $\theta_{1}=\left.\eta_{1}\right|_{S^{\prime \prime}}$

(3) let $D$ be a $\theta_{1}$-block and $E$ the corresponding $\theta_{2}$-block, then $D \times \operatorname{umax}(E) \subseteq S^{\prime}$; 
(4) for any $\mathbf{b} \in \mathcal{S}_{W_{2}}^{\prime \mathcal{R}}$ such that $\operatorname{pr}_{X \cup\left(W_{2}-W\right)} \mathbf{b}$ is u-maximal in a $\theta_{2}$-block and any $\mathbf{b}^{\prime} \in \mathcal{S}_{W \cap W_{2}}^{\prime \mathcal{R}}$ such that $\mathbf{b}^{\prime} \stackrel{\theta^{\prime}}{\equiv} \operatorname{pr}_{W \cap W_{2}} \mathbf{b}$ there is $\mathbf{b}^{\prime \prime} \in S$ such that $\operatorname{pr}_{W \cap W_{2}} \mathbf{b}^{\prime \prime} \stackrel{\tau}{\equiv} \mathbf{b}^{\prime}$ and $\operatorname{pr}_{X \cup\left(W_{2}-W\right)} \mathbf{b}^{\prime \prime}=\operatorname{pr}_{X \cup\left(W_{2}-W\right)} \mathbf{b}$.

(1) It follows by Proposition 35 for any $\theta_{1}$-block $D^{\prime}$ and a $\theta_{2}$-block $E^{\prime}$ with $S^{\prime} \cap\left(D^{\prime} \times E^{\prime}\right) \neq \varnothing$, that $D^{\prime} \times \operatorname{umax}\left(E^{\prime}\right) \subseteq S^{\prime}$.

Let $\mathbf{b} \in S, D^{\prime}$ be the $\theta_{1}$-block containing $\left(\operatorname{pr}_{W \cap W_{2}} \mathbf{b}\right)^{\tau}$ and $E^{\prime}$ the corresponding $\theta_{2}$-block. Then by the Maximality Lemma 27(5) there is $\mathbf{b}^{\prime} \in S$ such that $\operatorname{pr}_{W \cap W_{2}} \mathbf{b}^{\prime} \stackrel{\tau}{\equiv} \operatorname{pr}_{W \cap W_{2}} \mathbf{b}$ and $\operatorname{pr}_{H} \mathbf{b}^{\prime}$ is u-maximal in $E^{\prime}$. We assume that $\mathbf{b}$ satisfies this condition. Let also $D$ be the $\eta_{1}$-block containing $\left(\operatorname{pr}_{W \cap W_{2}} \mathbf{b}\right)^{\tau}$. Note that $D^{\prime} \subseteq D$.

Suppose there is $\mathbf{c} \in D$ such that $\left(\mathbf{c}^{\prime}, \operatorname{pr}_{H} \mathbf{b}\right) \in S$ for no $\mathbf{c}^{\prime} \in \mathbf{c}$. We will derive a contradiction. Take some u-maximal tuple $\mathbf{c}^{\prime}$ from $\mathbf{c}$. Since $\operatorname{pr}_{W \cap W_{2}} \mathbf{b} \not \equiv \mathbf{c}^{\prime}$, there is $C^{\prime}=\left\langle\mathbf{s}^{\prime}, R^{\prime}\right\rangle \in \mathcal{C}$ and $(u, \chi, \xi) \in \mathcal{W}(\bar{\beta})$ such that $\left(\operatorname{pr}_{Z} \mathbf{b}, \operatorname{pr}_{Z} \mathbf{c}^{\prime}\right) \notin \tau^{*}$, where $\tau^{*}=\tau_{C^{\prime}}^{\prime}(u, \chi \xi)$ and $Z=W_{u, \chi \xi} \cap W \cap W_{2} \cap \mathbf{s}^{\prime}$. Choose a pair b, c in such a way that the number of such constraints and triples is minimal. We will find a polynomial $f$ of $\mathcal{S}_{W_{2}}^{\prime}$ such that (roughly speaking) $f\left(\operatorname{pr}_{W \cap W_{2}} \mathbf{b}\right), f\left(\mathbf{c}^{\prime}\right) \in \operatorname{pr}_{W \cap W_{2}} \mathcal{S}_{W_{2}}^{\prime \mathcal{R}}$, $f\left(\operatorname{pr}_{H} \mathbf{b}\right)=\operatorname{pr}_{H} \mathbf{b}$, and $f\left(\operatorname{pr}_{W \cap W_{2}} \mathbf{b}\right), f\left(\mathbf{c}^{\prime}\right)$ differ on fewer constraints and triples. Let $\mathbf{b}^{\prime \prime}=\left(\operatorname{pr}_{Z} \mathbf{b}\right) \tau^{\tau^{*}}, \mathbf{c}^{\prime \prime}=\left(\operatorname{pr}_{Z} \mathbf{c}^{\prime}\right)^{\tau^{*}}$. Since the interval $\left(\tau^{*}, \bar{\beta}_{Z}\right)$ has type 2, $\left\{\mathbf{b}^{\prime \prime}, \mathbf{c}^{\prime \prime}\right\}$ is a $\left(\tau^{*}, \bar{\beta}_{Z}\right)$-subtrace. Let $\left(x, \gamma_{x}, \delta_{x}\right) \in \mathcal{W}(\bar{\beta}), x \in Z$, be such that $\left(\alpha, \beta_{v}\right)$ and $\left(\gamma_{x}, \delta_{x}\right)$ cannot be separated in $R^{\{v, x\}}$.

By (S7) for $\mathcal{R}$ relation $\mathcal{S}_{W_{2}}^{\prime}$ is strongly chained. Since $\tau^{*} \prec \bar{\beta}^{\prime \prime}$ on $\operatorname{pr}_{Z} \mathcal{S}_{W_{2}}^{\prime \mathcal{R}}$ if we consider $\mathcal{S}_{W_{2}}^{\prime}$ as a subdirect product of $\operatorname{pr}_{Z} \mathcal{S}_{W_{2}}^{\prime}$ and $\mathbb{A}_{y}, y \in W_{2}-Z$, by Lemma 48 there is a $\left(\tau^{*}, \bar{\beta}_{Z}\right)$-collapsing polynomial $f$ of $\mathcal{S}_{W_{2}}^{\prime}$ for $\bar{\beta}, \bar{B}$. By Lemma $74\left(\tau^{*}, \bar{\beta}_{Z}\right)$ cannot be separated from $\left(\gamma_{x}, \delta_{x}\right)$ for $x \in W \cap W_{2}$. For any $(z, \eta, \theta) \in \mathcal{W}(\bar{\beta}), z \in W_{2}$, consider $\operatorname{pr}_{Z \cup\{z\}} \mathcal{S}_{W_{2}}^{\prime}$. If $(\eta, \theta)$ can be separated from $\left(\alpha, \beta_{v}\right)$ (or $\left(\alpha, \beta_{v}\right)$ can be separated from $\left.(\eta, \theta)\right)$ then $\left(\tau^{*}, \bar{\beta}_{Z}\right)$ can be separated from $(\eta, \theta)$ (or the other way round). In particular, by Lemma $48 f$ can be chosen to satisfy the following conditions

(a) $f\left(\operatorname{pr}_{Z}\left(\mathcal{S}_{W_{2}}^{\prime}\right)\right)$ is a $\left(\tau^{*}, \bar{\beta}_{Z}\right)$-minimal set and $f\left(\mathbb{A}_{y}\right)$ is a $\left(\gamma_{y}, \delta_{y}\right)$-minimal set for $y \in W \cap W_{2}$;

(b) for every $z \in W_{2}-W,\left|f\left(B_{z}\right)\right|=1$; and

(c) $f$ is idempotent.

Since $\left\{\mathbf{b}^{\prime \prime}, \mathbf{c}^{\prime \prime}\right\}$ is a $\left(\tau^{*}, \bar{\beta}_{Z}\right)$-subtrace of $\left(\mathrm{pr}_{Z} R_{C^{\prime}, u, \chi \xi}\right) / \tau^{*}$, by condition (Q2s) of being strongly chained (S7) for $\mathcal{R}$ polynomial $f$ can be chosen such that

(d) $\mathbf{b}^{\prime \prime}=f\left(\mathbf{b}^{\prime \prime}\right), \mathbf{c}^{\prime \prime}=f\left(\mathbf{c}^{\prime \prime}\right)$.

Moreover, let $\mathbf{b}^{*} \in \operatorname{pr}_{W \cap W_{2}} \mathcal{S}_{W_{2}}^{\prime \mathcal{R}}$ be such that there is a tuple $\mathbf{d} \in \mathcal{S}_{W_{2}}^{\prime \mathcal{R}}$ such that 
$\operatorname{pr}_{W_{2}-W} \mathbf{d}=\operatorname{pr}_{W_{2}-W} \mathbf{b}, \operatorname{pr}_{W \cap W_{2}} \mathbf{d}=\mathbf{b}^{*}, \mathrm{pr}_{Z} \mathbf{b}^{*} \in \mathbf{b}^{\prime \prime}$, and $\mathbf{d}$ is u-maximal in $\mathcal{S}_{W_{2}}^{\prime} \cap \bar{B}$. Such a tuple exists. Indeed, for any $\left(z, \gamma^{\prime}, \delta^{\prime}\right) \in \mathcal{W}(\bar{\beta}), z \in W_{2}-$ $W$, and any $x \in W \cap W_{2}$, the interval $\left(\gamma_{x}, \delta_{x}\right)$ and therefore $\left(\tau^{*}, \bar{\beta}^{\prime \prime}\right)$ can be separated from $\left(\gamma^{\prime}, \delta^{\prime}\right)$, or the other way round. Lemma 48 also implies that for any $\gamma^{\prime}, \delta^{\prime} \in \operatorname{Con}\left(\mathcal{S}_{W_{2}-W}^{\prime}\right), \gamma^{\prime} \prec \delta^{\prime} \leq \bar{\beta}_{W_{2}-W}$, the interval $\left(\tau^{*}, \bar{\beta}^{\prime \prime}\right)$ can be separated from $\left(\gamma^{\prime}, \delta^{\prime}\right)$, or the other way round. Therefore, by the Congruence Lemma 64 $\operatorname{pr}_{W \cap W_{2}} \mathcal{S}_{W_{2}}^{\prime \mathcal{R}} / \tau \times \operatorname{umax}\left(\operatorname{pr}_{W_{2}-W} \mathcal{S}_{W_{2}}^{\prime \mathcal{R}}\right) \subseteq \mathcal{S}_{W_{2}}^{\prime \mathcal{R}}$. By Lemma 57 polynomial $f$ can be chosen such that

(e) $f(\mathbf{d})=\mathbf{d}$.

Let $\mathbf{c}^{\prime}$ be a u-maximal tuple from the $\tau$-block $\mathbf{c} \subseteq \mathcal{S}_{W \cap W_{2}}^{\prime \mathcal{R}}$, and let $\mathbf{c}^{*}=$ $f\left(\mathbf{c}^{\prime}\right)$, and $\mathbf{c}^{\dagger}$ a tuple from $\operatorname{Sg}\left(\mathbf{b}^{*}, \mathbf{c}^{*}\right)$ such that $\mathbf{b}^{*} \sqsubseteq$ as $\mathbf{c}^{\dagger}$ and $\mathbf{c}^{*} \stackrel{\tau}{\equiv} \mathbf{c}^{\dagger}$; such a tuple $\mathbf{c}^{\dagger}$ exists, because $\mathcal{S}_{W \cap W_{2}}^{\mathcal{R}} / \tau$ is a module. Note that it suffices to prove that $\varrho=\left(\mathbf{c}^{\dagger}, \operatorname{pr}_{W_{2}-W} \mathbf{b}\right) \in \mathcal{S}_{W_{2}}^{\prime \mathcal{R}}$. Indeed, since $\operatorname{pr}_{Z} \mathbf{c}^{*} \stackrel{\tau^{*}}{\equiv} \operatorname{pr}_{Z} \mathbf{c}^{\prime}$ and $\mathbf{c}^{\prime}$ agrees with $\mathbf{c}^{\dagger}$ modulo $\tau_{C^{\prime \prime}}^{\prime}\left(u, \chi^{\prime} \xi^{\prime}\right)$ for every $C^{\prime \prime} \in \mathcal{C}$ and $\left(u^{\prime}, \chi^{\prime} \xi^{\prime}\right) \in \mathcal{W}(\bar{\beta})$, for which $\mathbf{b}$ and $\mathbf{c}^{\prime}$ agree, we obtain a contradiction with the choice of $\mathbf{b}, \mathbf{c}$.

To show that $\varrho$ is an $\mathcal{R}$-compatibile solution take any $C^{\prime \prime}=\left\langle\mathbf{s}^{\prime \prime}, R^{\prime \prime}\right\rangle \in \mathcal{C}$ and $\left(x, \chi^{\prime}, \xi^{\prime}\right) \in \mathcal{W}(\bar{\beta})$ and let $U^{\prime \prime}=\mathbf{s}^{\prime \prime} \cap W_{2} \cap W_{x, \chi^{\prime} \xi^{\prime}}$. We show that $\varrho\left(U^{\prime \prime}\right) \in$ $\operatorname{pr}_{U^{\prime \prime}} R_{C^{\prime \prime}, x, \chi^{\prime} \xi^{\prime}}$; note that this proves not only $\mathcal{R}$-compatibility, but also that $\varrho$ is a solution: $\left(x, \chi^{\prime}, \xi^{\prime}\right)$ can be chosen to be $\left(w_{2}, \gamma_{2}, \delta_{2}\right)$. Let $U^{\prime}=W^{\prime} \cap W$. Since $\mathbf{c}^{\prime} \in$ $\operatorname{umax}\left(\mathcal{S}_{W \cap W_{2}}^{\prime \mathcal{R}}\right)$, we have $\operatorname{pr}_{U^{\prime}} \mathbf{c}^{\prime} \in \operatorname{umax}\left(\operatorname{pr}_{U^{\prime}} R_{C^{\prime \prime}, x, \chi^{\prime} \xi^{\prime}}\right)$. By (S2) for $\mathcal{R}$ tuple $\operatorname{pr}_{U^{\prime}} \mathbf{c}^{\prime}$ can be extended to an $\mathcal{R}$-compatible solution $\sigma$ from $\mathcal{S}_{W_{2}}^{\prime}$. By the choice of $f$, property (b), $f\left(\operatorname{pr}_{U^{\prime \prime}-U^{\prime}} \sigma\right)=\operatorname{pr}_{U^{\prime \prime}-U^{\prime}} \mathbf{d}=\operatorname{pr}_{U^{\prime \prime}-U^{\prime}} \mathbf{b}$, and $f\left(\operatorname{pr}_{U^{\prime}} \sigma\right)=\operatorname{pr}_{U^{\prime}} \mathbf{c}^{*}$ by definition of $\mathbf{c}^{*}$. Since $\operatorname{pr}_{U^{\prime}} \mathbf{b}^{*} \sqsubseteq$ as $\operatorname{pr}_{U^{\prime}} \mathbf{c}^{\dagger}$ in $\operatorname{Sg}\left(\operatorname{pr}_{U^{\prime}} \mathbf{b}^{*}, \operatorname{pr}_{U^{\prime}} \mathbf{c}^{*}\right)$, we have $\operatorname{pr}_{U^{\prime \prime}} \mathbf{b}^{*} \sqsubseteq$ as $\operatorname{pr}_{U^{\prime \prime}} \varrho$ in $\operatorname{Sg}\left(\mathrm{pr}_{U^{\prime \prime}} \mathbf{b}^{*}, \mathrm{pr}_{U^{\prime \prime}} \mathbf{c}^{*}\right)$, implying by (S6) for $\mathcal{R}$ that $\operatorname{pr}_{U^{\prime \prime}} \varrho \in$ $R_{C^{\prime \prime}, x, \chi^{\prime} \xi^{\prime}}$. As this is true for every constraint $C^{\prime \prime}, \varrho$ is an $\mathcal{R}$-compatible solution.

(2) The proof of item (1) shows in particular that for any $\mathbf{b} \in S$ and $\mathbf{c} \in$ $\left(\operatorname{pr}_{W \cap W_{2}} S\right) / \tau$ such that $\mathbf{c} \stackrel{\eta_{1}}{=}\left(\operatorname{pr}_{W \cap W_{2}} \mathbf{b}\right)^{\tau}$, we also have $\left(\operatorname{pr}_{W \cap W_{2}} \mathbf{b}\right)^{\tau} \stackrel{\theta_{1}}{\equiv} \mathbf{c}$, as both $\mathbf{b}^{\tau}$ and $\left(\mathbf{c}, \mathrm{pr}_{H} \mathbf{b}\right)$ belong to $S^{\prime}$.

(3) follows from Proposition 35 .

(4) follows from (1) and (3).

We now can complete the proof of Lemma 80. If we show that a can be extended to $\mathbf{b} \in S$ such that $\operatorname{pr}_{U} \mathbf{b}=\mathbf{a}$ and such that $\operatorname{pr}_{H} \mathbf{b}$ is u-maximal in a $\theta_{2}$-block, Claim 2 implies (S2) for $\mathcal{R}^{\prime}$. Indeed, suppose there is $\mathbf{b} \in S$ satisfying the above conditions. Since $\operatorname{pr}_{X} \mathbf{a} \in \operatorname{pr}_{X} \mathcal{S}_{W}^{\prime \mathcal{R}^{\prime}} \subseteq \operatorname{pr}_{X} \mathcal{S}_{W \cap W_{2}}^{\prime \mathcal{R}^{\prime}}$, there is $\mathbf{c}^{\prime} \in \mathcal{S}_{W \cap W_{2}}^{\prime \mathcal{R}^{\prime}}=\operatorname{pr}_{W \cap W_{2}} S$ such that $\operatorname{pr}_{X} \mathbf{a}=\operatorname{pr}_{X} \mathbf{c}^{\prime}$. In particular, this means that $\mathbf{c}=\mathbf{c}^{\prime \tau} \in D$, where $D$ is the $\eta_{1^{-}}$, and therefore $\theta_{1}$-block containing $\left(\operatorname{pr}_{W \cap W_{2}} \mathbf{b}\right)^{\tau}$, because $\operatorname{pr}_{X} \mathbf{c}^{\prime}=\operatorname{pr}_{X} \mathbf{b}=\operatorname{pr}_{X} \mathbf{a}$. Then as $\left(\mathbf{c}, \operatorname{pr}_{H} \mathbf{b}\right) \in S^{\prime}$ by Claim 2(4), this 
means that there is $\mathbf{d} \in \operatorname{umax}\left(\mathcal{S}_{W_{2}}^{\prime \mathcal{R}}\right)$ such that $\operatorname{pr}_{U} \mathbf{d}=\mathbf{a}$ and $\operatorname{pr}_{W \cap W_{2}} \mathbf{d} \stackrel{\tau}{\equiv} \mathbf{c}^{\prime}$; that is, $\mathbf{d} \in \mathcal{S}_{W_{2}}^{\prime \mathcal{R}^{\prime}}$.

Next we show such $\mathbf{b}$ exists. Recall that $S^{*}$ denotes the $\tau$-block of $\operatorname{pr}_{W \cap W_{2}} Q=$ $\mathcal{S}_{W \cap W_{2}}^{\prime \mathcal{R}}$ that contains $\mathcal{S}_{W \cap W_{2}}^{\prime \mathcal{R}^{\prime}}$, and in particular, $\operatorname{pr}_{W \cap W_{2}} \mathcal{S}_{W}^{\prime \mathcal{R}^{\prime}}$. First, observe that

$$
S^{*} \cap \operatorname{pr}_{W \cap W_{2}} S=S^{*} \cap \operatorname{pr}_{W \cap W_{2}} \mathcal{S}_{W_{2}}^{\mathcal{R}} \neq \varnothing .
$$

Indeed, let $\mathbf{d} \in S$ be such that $\operatorname{pr}_{U} \mathbf{d}=\mathbf{a}$. Then $\operatorname{pr}_{W \cap W_{2}} \mathbf{d} \in \mathcal{S}_{W \cap W_{2}}^{\prime \mathcal{R}}$ and $\mathbf{d}^{\tau}$ belongs to the same $\eta_{1}$-block as $S^{*}$, because $\left(S^{*} / \tau, \operatorname{pr}_{X} \mathbf{a}\right) \in Q^{\prime}$. By Claim 2(1) $S^{*} /_{\tau} \in\left(\operatorname{pr}_{W \cap W_{2}} S\right) /_{\tau}$, and as $\operatorname{pr}_{W \cap W_{2}} S \subseteq \mathcal{S}_{W \cap W_{2}}^{\prime \mathcal{R}}$, this proves the observation.

Let $D$ be the $\theta_{1}$-block containing $S^{*} / \tau$ and $E$ the corresponding $\theta_{2}$-block. By what is proved above $\operatorname{pr}_{H} \mathbf{d} \in E$. We now only need to show that $\mathbf{d}$ can be chosen such that $\operatorname{pr}_{H} \mathbf{d} \in \operatorname{umax}(E)$. Let $\pi$ be the congruence on $\operatorname{pr}_{H} S$ given by $\mathbf{e}_{1} \stackrel{\pi}{=} \mathbf{e}_{2}$ if and only if $\operatorname{pr}_{X^{\prime}} \mathbf{e}_{1} \stackrel{\tau_{X}}{=} \operatorname{pr}_{X^{\prime}} \mathbf{e}_{2}$. Then $\operatorname{pr}_{H} S / \pi$ is isomorphic to $\operatorname{pr}_{X}\left(R_{C, w_{1}, \gamma_{1} \delta_{1}}\right) / \tau_{X}$, in particular, it is a module if $\tau_{X}$ is maximal, and 1-element otherwise. Let $\tau_{X}^{\prime}$ denote the congruence of $\operatorname{pr}_{U} R_{C, w_{1}, \gamma_{1} \delta_{1}}$ given by $\mathbf{c} \stackrel{\tau_{X}^{\prime}}{=} \mathbf{d}$ if and only if $\operatorname{pr}_{X} \mathbf{c} \stackrel{\tau_{X}}{=} \operatorname{pr}_{X} \mathbf{d}$. As is easily seen, if $G$ is a $\pi$-block of $\operatorname{pr}_{H} S$ then $\operatorname{pr}_{U} G$ is a $\tau_{X}^{\prime}$-block of $\operatorname{pr}_{U} R_{C, w_{1}, \gamma_{1} \delta_{1}}$. Therefore, as a is u-maximal in $\operatorname{pr}_{U} R_{C, w_{1}, \gamma_{1} \delta_{1}}^{\prime}$, by the Maximality Lemma27 (5) it can be extended to a u-maximal tuple $\mathbf{a}^{\prime}$ in a $\pi$-block $G$. Since $E / \pi$ is a module, by Lemma $26 \mathbf{a}^{\prime}$ is also u-maximal in $E$. The result follows.

Condition (S3) can be proved in a similar way, so we just indicate where the proof of Lemma 80 has to be changed.

Lemma $82 \mathcal{R}^{\prime}$ satisfies condition (S3). That is, for every $C=\langle\mathbf{s}, R\rangle \in \mathcal{C}, R \cap$ $\bar{B}_{\mathrm{s}}^{\prime} \neq \varnothing$ and for any $I \subseteq \mathrm{s}$ any $\mathcal{R}^{\prime}$-compatible tuple $\mathbf{a} \in \operatorname{umax}\left(\operatorname{pr}_{I} R\right)$ extends to an $\mathcal{R}^{\prime}$-compatible tuple $\mathbf{b} \in R$.

Proof: If $\mathbf{s} \cap W=\varnothing$ then Corollary 75 implies that every u-maximal $\mathcal{R}$ compatible tuple is also $\mathcal{R}^{\prime}$-compatible, and the result follows from (S3) for $\mathcal{R}$. Suppose that $W^{\prime}=\mathbf{s} \cap W \neq \varnothing$. Then, as there is a $\mathcal{R}^{\prime}$-compatible tuple in $\operatorname{umax}\left(\operatorname{pr}_{w} R\right)$ for $w \in W^{\prime}$, the first part of (S3) follows from the second part.

To prove the second part we show that $R, R^{\mathcal{R}}$ satisfy conditions (X1)-(X5), and therefore the argument from the proof of Lemma 80 applies in this case as well. We take s for $W_{2}$ and set $Y=\varnothing$. Condition (X1) is trivial; condition (X2) follows from (S3) for $\mathcal{R}$; and congruences $\tau_{C^{\prime}}^{\prime}(u, \eta \theta)$ can be defined in the same way replacing $W_{2}$ with $\mathbf{s}$, and they possess the same properties required in (X3). Conditions (X4),(X5) follow from the definitions. Now we use the same argument except that the proof of Claim 1 in this case is simpler, because we do not need to show something is a solution; just that the required tuple belongs to a relation. 


\subsubsection{The remaining conditions}

We can now complete the proof of Theorem 76

Proof: (of Theorem 76). Conditions (S1)-(S3) and (S6) are proved in the preceding lemmas.

For (S4) observe that every $R_{C, w, \gamma \delta}^{\prime}$ is obtained as the intersection of $R_{C, w, \gamma \delta}$ with a block of $\tau_{C, w, \gamma \delta}$, and therefore is a subalgebra. Also, since $R_{C, w, \gamma \delta} / \tau_{C, w, \gamma \delta}$ is a module, by Lemma $26 \operatorname{umax}\left(R_{C, w, \gamma \delta}^{\prime}\right) \subseteq \operatorname{umax}\left(R_{C, w, \gamma \delta}\right)$ proving the first part of (S4).

To prove the rest of (S4) let $(w, \gamma, \delta) \in \mathcal{W}(\bar{\beta}), U=W_{w, \gamma \delta}$ and $\mathcal{S}_{U}^{\prime}$ be one of the sets $\mathcal{S}_{U}$ if $(w, \gamma, \delta) \notin \mathcal{W}^{\prime}$, or $\mathcal{S}_{U, Y}$ for $Y=\operatorname{MAX}(\mathcal{P})-\mathbf{s}$ for some $C=$ $\langle\mathbf{s}, R\rangle$ or $Y=\operatorname{MAX}(\mathcal{P})-W_{u, \eta \theta}$ for some $(u, \eta, \theta) \notin \mathcal{W}^{\prime}$, if $(w, \gamma, \delta) \in \mathcal{W}^{\prime}$. As in the proof of condition (S2) we consider the congruence $\tau$ constructed as in (1) with $U$ in place of $W_{2}$. Let $Q$ be the $\tau$-block of $\operatorname{pr}_{W \cap U} \mathcal{S}_{U}^{\prime}$ containing $\mathcal{R}^{\prime}$ compatible tuples. By (S4) for $\mathcal{R}$ there is a tuple $\mathbf{a} \in \mathcal{S}_{U}^{\prime \mathcal{R}}$ that is in $\operatorname{umax}\left(\mathcal{S}_{U}^{\prime}\right)$. Since $\operatorname{pr}_{W \cap U} \mathcal{S}_{U}^{\prime} / \tau$ is a module, by the Maximality Lemma 27(4) and Lemma 22 there is an as-path $\mathbf{a}=\mathbf{a}_{1}, \ldots, \mathbf{a}_{k}$ in $\mathcal{S}_{U}^{\prime \mathcal{R}}$ such that $\operatorname{pr}_{W \cap U} \mathbf{a}_{k} \in Q$. The tuple $\mathbf{a}_{k}$ belongs to $\mathcal{S}_{U}^{\prime \mathcal{R}^{\prime}}$ and to umax $\left(\mathcal{S}_{U}^{\prime}\right)$.

For (S5), the existence of $A_{\mathcal{R}^{\prime}, w}$ follows from (S3). Also, as in the proof of (S4) $\operatorname{umax}\left(A_{\mathcal{R}^{\prime}, w}\right) \subseteq \operatorname{umax}\left(A_{\mathcal{R}, w}\right)$. The result now follows from (S5) for $\mathcal{R}$.

Finally, (S7) follows from Lemma 65.

\subsection{Tightening non-affine factors}

Let $\mathcal{P}=(V, \mathcal{C})$ be a block-minimal instance, let $\mathcal{R}$ be a $\bar{\beta}$-strategy with respect to $\bar{B}$. Take $v \in V$ and $\alpha \in \operatorname{Con}\left(\mathbb{A}_{v}\right)$ with $\alpha \prec \beta_{v}$ such that $\operatorname{typ}\left(\alpha, \beta_{v}\right) \neq \mathbf{2}$. We tighten $\mathcal{R}$ in two steps. In the first step we restrict $B_{v}$ to the subalgebra generated by an as-component of $A_{\mathcal{R}, v}$ obtaining a collection of relations that satisfies all the properties of a strategy except (S5) and (S6). In the second step we restrict the same domain to one $\alpha$-block and restore (S5) and (S6). Let $D$ be an as-component of $A_{\mathcal{R}, v} / \alpha$, by (S5) $D$ is also an as-component of $B_{v} / \alpha$. Note that if $\operatorname{typ}\left(\alpha, \beta_{v}\right) \in$ $\{\boldsymbol{4}, \boldsymbol{5}\}$ then by Lemma 40 any as-component of $B_{v} / \alpha$ is a singleton and Step 2 is not needed. Conditions (S5),(S6) in this case are proved as in Step 2.

\subsubsection{Step 1.}

Let $D^{\prime}=\left\{a \in A_{\mathcal{R}, v} \mid a^{\alpha} \in D\right\}$ and let $\widehat{D}=\operatorname{Sg}\left(D^{\prime}\right)$. We consider the problem $\mathcal{P}^{\prime}$ obtained from $\mathcal{P}$ by restricting the domain of $v$ to $\widehat{D}$ and the domain of $w \in V-\{v\}$ to $A_{\mathcal{R}, w}$. We first show that $\mathcal{P}^{\prime}$ can be converted to a nonempty $(2,3)$-minimal instance that also satisfies some additional conditions. 
In order to do that we introduce a family of binary relations, and then prove that this family is a $(2,3)$-strategy of $\mathcal{P}^{\prime}$. For $x, y \in V$, let

$$
\begin{aligned}
& Q^{x}=\left\{a \in \operatorname{amax}\left(A_{\mathcal{R}, x}\right) \mid \text { there is } d \in D \text { such that }(d, a) \in R^{\{v, x\}, \mathcal{R}} / \alpha\right\}, \\
& \text { and } \\
& \begin{array}{c}
Q^{x y}= \\
\quad(d, b) \in \operatorname{amax}\left(R^{\{x, y\}, \mathcal{R}}\right) \mid \text { there is } d \in D \text { such that } \\
\left.\quad(d, a) \in R^{\{v, x\}, \mathcal{R}} / \alpha,(d, b) \in R^{\{v, y\}, \mathcal{R}} / \alpha\right\} .
\end{array}
\end{aligned}
$$

In particular $Q^{v}=\operatorname{amax}\left(D^{\prime}\right)$. We say that a tuple a on a set $U \subseteq V$ (where $U$ can be, e.g. a subset of $\mathbf{s}$ for a constraint $C=\langle\mathbf{s}, R\rangle$, or a subset of $W_{w, \gamma \delta}$ for some $(w, \gamma, \delta) \in \mathcal{W}(\bar{\beta}))$ is $Q$-compatible if $(\mathbf{a}[x], \mathbf{a}[y]) \in Q^{x y}$ for any $x, y \in U$.

Proposition 83 (1) For any $x, y, z \in V$ and any $(a, b) \in Q^{x y}$ there is $c \in$ $\operatorname{amax}\left(A_{\mathcal{R}, z}\right)$ such that $(a, c) \in Q^{x z}$ and $(b, c) \in Q^{y z}$.

(2) For any $C=\langle\mathbf{s}, R\rangle$ let $R^{\mathcal{R}}$ denote the set of $\mathcal{R}$-compatible tuples from $R$. For any $I \subseteq \mathbf{s}$ and any $Q$-compatible $\mathbf{a} \in \operatorname{amax}\left(\operatorname{pr}_{I} R^{\mathcal{R}}\right)$, there is $\mathbf{a}^{\prime} \in \operatorname{amax}\left(R^{\mathcal{R}}\right)$ that is $Q$-compatible, and $\operatorname{pr}_{I} \mathbf{a}^{\prime}=\mathbf{a}$.

(3) For any $(w, \gamma, \delta) \in \mathcal{W}(\bar{\beta})$, any $U \subseteq W_{w, \gamma \delta}$, and any $\mathbf{a} \in \operatorname{amax}\left(\operatorname{pr}_{U} \mathcal{S}_{W_{w, \gamma \delta}}^{\prime \mathcal{R}}\right)$, where $\mathcal{S}_{W_{w, \gamma \delta}}^{\prime \mathcal{R}}$ is the set of solutions of $\left(\mathcal{P}_{W_{w, \gamma \delta}}\right) / \bar{\mu}^{Y}$ for some set $Y$ from the definition of block-minimality, there is $\mathbf{a}^{\prime} \in \operatorname{amax}\left(\mathcal{S}_{W_{w, \gamma \delta}^{\prime}}^{\mathcal{R}}\right)$ that is $Q$-compatible and $\operatorname{pr}_{U} \mathbf{a}^{\prime}=\mathbf{a}$.

Proof: For $x, y \in V$ let $Q^{v x y}$ denote the set of tuples $(d, a, b)$ such that $(d, a) \in$ $R^{\{v, x\}, \mathcal{R}} / \alpha,(d, b) \in R^{\{v, y\}, \mathcal{R}} / \alpha,(a, b) \in R^{\{x, y\}, \mathcal{R}}$.

Claim 1. The set $Q^{x}$ is as-closed in $A_{R, x}$, and $Q^{x y}$ is as-closed if $R^{\{x, y\}, \mathcal{R}}$.

Let $(a, b) \in Q^{x y}$. By the Maximality Lemma 27(3) either $Q^{v x y}$ contains a subdirect product of $D$ and as $(a, b)$, or $(D \times$ as $(a, b)) \cap Q^{v x y}=\varnothing$. Since $(a, b) \in Q^{x y}$ the former option holds. For the first part of the claim observe that $\operatorname{pr}_{x}$ as $(a, b)=\operatorname{as}(a)$.

Claim 2. For any $x, y \in V, Q^{x y}$ is a subdirect product of $Q^{x} \times Q^{y}$.

Let $a \in Q^{x}$, then there is $d \in D$ with $(d, a) \in R^{\{v, x\}, \mathcal{R}}$. By (S1) for $\mathcal{R}$, $\left\{\operatorname{umax}\left(R^{X, \mathcal{R}}\right)\right\}_{X \subseteq V,|X|=2}$ is (2,3)-strategy, and there is $b \in \mathbb{A}_{y}$ with $(d, a, b) \in$ $Q^{v x y}$; then $(a, b) \in Q^{x y}$.

We prove (2), the proof of (3) is basically identical, and we explain how to modify this proof to prove (1).

By induction on $i$ we prove that a Q-compatible tuple $\mathbf{a} \in \operatorname{amax}\left(\operatorname{pr}_{I} R^{\mathcal{R}}\right)$ can be found for any $I \subseteq \mathbf{s},|I|=i$. Moreover, for any Q-compatible $\mathbf{a}^{\prime} \in$ $\operatorname{amax}\left(\operatorname{pr}_{I-\{u\}} R^{\mathcal{R}}\right)$, a Q-compatible $\mathbf{b} \in \operatorname{amax}\left(\operatorname{pr}_{I} R^{\mathcal{R}}\right)$ can be found such that $\operatorname{pr}_{I-\{u\}} \mathbf{b}=\mathbf{a}^{\prime}$. 
First we consider the case $u \neq v$. For $i=2$ the existence of a follows from Claim 2. So, suppose such a tuple exists for any $I \subseteq \mathrm{s}$ with $|I| \leq i$. Let $I \subseteq \mathrm{s}$, $|I|=i+1, y \in I, I^{\prime}=I-\{y\}$, and $\mathbf{a} \in \operatorname{amax}\left(\operatorname{pr}_{I^{\prime}} R^{\mathcal{R}}\right)$ is Q-compatible. Let also $t \in I^{\prime}, I^{\prime \prime}=I^{\prime}-\{t\}$. Without loss of generality assume $I=\left\{x_{1}, \ldots, x_{i+1}\right\}$, $y=x_{i+1}, t=x_{i}$. Consider the relation given by

$$
\begin{aligned}
& Q\left(x_{1}, \ldots, x_{i}, z_{1}, \ldots, z_{i}\right)=\exists y \operatorname{pr}_{I^{\prime \prime}} R^{\mathcal{R}}\left(x_{1}, \ldots, x_{i-1}\right) \\
& \quad \wedge \bigwedge_{j=1}^{i}\left(R^{\left\{x_{j}, v\right\}, \mathcal{R}} / \alpha\left(x_{j}, z_{j}\right) \wedge R^{\left\{v, x_{i+1}\right\}, \mathcal{R}} / \alpha\left(z_{j}, y\right) \wedge R^{\left\{x_{j}, x_{i+1}\right\}, \mathcal{R}}\left(x_{j}, y\right)\right) .
\end{aligned}
$$

It suffices to prove that $\mathbf{a}^{\prime \prime}=(\mathbf{a}, \mathbf{e}) \in Q$, where $\mathbf{e}[j] \in D$ for each $j \in[i]$, since this would mean that there is a $c \in \mathbb{A}_{\mathcal{R}, x_{i+1}}$ with the required properties.

Observe first that $\operatorname{umax}\left(\operatorname{pr}_{I^{\prime}} R^{\mathcal{R}}\right) \subseteq \operatorname{pr}_{I^{\prime}} Q$. Indeed, any $\mathbf{b} \in \operatorname{pr}_{I^{\prime}} R^{\mathcal{R}}$ by (S3) can be extended to $\mathbf{b}^{\prime} \in \operatorname{pr}_{I} R^{\mathcal{R}}$; then the values of the variables $z_{j}$ can be chosen by $(2,3)$-consistency and (S1). This also implies that umax $\left(\operatorname{pr}_{I^{\prime \prime}} Q\right)=$ umax $\left(\operatorname{pr}_{I^{\prime \prime}} R^{\mathcal{R}}\right)$. Since $\alpha \prec \beta_{v}$, by (S7) for $\mathcal{R}$ and the Congruence Lemma 64 for any $w \in V$ the relation $R^{\{v, w\}, \mathcal{R}} / \alpha$ either contains $D \times \operatorname{umax}\left(Q^{w}\right)$, or $R^{\{v, w\}, \mathcal{R}} /{ }_{\alpha} \cap$ $\left(D \times Q^{w}\right)$ is the graph of a mapping $\kappa_{w}: Q^{w} \rightarrow D$. Let the set of variables for which the latter option holds be denoted by $Z$. By construction, for any $w_{1}, w_{2} \in Z$, and any $(c, d) \in Q^{w_{1} w_{2}}$, we have $\kappa_{w_{1}}(c)=\kappa_{w_{2}}(d)$. For any $w \in V-Z$, the set $Q^{w}$ is as-closed, and $D \times \operatorname{umax}\left(Q^{w}\right) \subseteq R^{\{v, w\}, \mathcal{R}} / \alpha$.

Let $J=I^{\prime} \cap Z$ and, if $J \neq \varnothing$, let $d=\kappa_{w}(\mathbf{a}[w])$ for any $w \in J$. If $J=\varnothing$, but $x_{i+1} \in Z$, then let $\left(\operatorname{pr}_{I^{\prime \prime}} \mathbf{a}, c\right)$ be an extension of $\operatorname{pr}_{I^{\prime \prime}}$ a to a $Q$-compatible tuple from $\operatorname{pr}_{I \cup\left\{x_{i+1}\right\}} R$ and set $d=\kappa_{x_{i+1}}(c)$. If $x_{i+1} \notin Z$, then let $d$ be any element of $D$. Then $d$ is such that $(\mathbf{a}[w], d) \in R^{\{w, v\}, \mathcal{R}} / \alpha$ for any $w \in I^{\prime}$.

Consider the tuple $\mathbf{b}=(\mathbf{a}, d, \ldots, d)$; we show that it satisfies the conditions of the 2-Decomposition Theorem 30 with $X=I^{\prime \prime}$. Note that we cannot replace $I^{\prime \prime}$ with $I^{\prime}$ here, because in order to apply the 2-Decomposition Theorem 30 a has to be as-maximal $\operatorname{pr}_{I^{\prime}} Q$, which may not be true. By what is observed before, $\operatorname{pr}_{I^{\prime \prime}} \mathbf{a} \in \operatorname{pr}_{I^{\prime \prime}} Q$, and for any $s_{1}, s_{2} \in I^{\prime}$ we have $\left(\mathbf{a}\left[s_{1}\right], \mathbf{a}\left[s_{2}\right]\right) \in \operatorname{pr}_{s_{1} s_{2}} Q$. We now show that for any of the remaining pairs of variables $x, z \in\left\{x_{1}, \ldots, x_{i}, z_{1}, \ldots, z_{i}\right\}$ $(\mathbf{b}[x], \mathbf{b}[z]) \in \operatorname{pr}_{x z} Q$. Let $x \in\left\{x_{1}, \ldots, x_{i}\right\}$ and $z \in\left\{z_{1}, \ldots, z_{i}\right\}$. If $x \neq x_{i}$, then by the inductive hypothesis $\operatorname{pr}_{I^{\prime \prime}}$ a can be extended to some value $c$ of $y$ such that $(\mathbf{a}[s], c) \in Q^{s y}$ for any $s \in I^{\prime \prime}$. Then there is $c^{\prime} \in \mathbb{A}_{x_{i}}$ such that $\left(c^{\prime}, c\right) \in Q^{x_{i} y}$. Also, $(c, d) \in Q^{y v}$ and $c^{\prime}$ can be chosen such that $\left(c^{\prime}, d\right) \in Q^{x_{i} v}$. If $x=x_{i}$, then find a value $c$ for $y$ such that $\left(\mathbf{a}\left[x_{i}\right], c\right) \in Q^{x_{i}, x_{i+1}}$, and then extend $c$ to a tuple on $I^{\prime \prime} \cup\{y\}$ by induction hypothesis. The values of $z_{j}$ can be set using $(2,3)$ consistency. Finally, if $x, z \in\left\{z_{1}, \ldots, z_{i}\right\}$, we proceed as in one of the previous cases. 
By the 2-Decomposition Theorem 30 there is $\mathbf{b}^{\prime} \in Q$ such that $\operatorname{pr}_{I^{\prime \prime}} \mathbf{a} \sqsubseteq$ as $\operatorname{pr}_{I^{\prime \prime}} \mathbf{b}^{\prime}$ in $Q^{\prime}=\operatorname{pr}_{I^{\prime \prime}} Q,(\mathbf{a}[x], \mathbf{a}[z]) \sqsubseteq a s\left(\mathbf{b}^{\prime}[x], \mathbf{b}^{\prime}[z]\right)$ in $R^{\{x, z\}, \mathcal{R}}$ for any $x, z \in I^{\prime}$, and $d \sqsubseteq_{a s} \mathbf{b}^{\prime}\left[z_{j}\right]$ in $A_{\mathcal{R}, v} / \alpha$ for any $j \in[i]$.

Let $\mathrm{Ik}_{1}, \mathrm{lk}_{2}$ be the link congruences of $Q^{\prime}, \mathbb{A}_{x_{i}}$ with respect to $\mathrm{pr}_{I^{\prime}} Q$. Since $\operatorname{umax}\left(\operatorname{pr}_{I^{\prime}} R^{\mathcal{R}}\right) \subseteq \operatorname{pr}_{I^{\prime}} Q$, the link congruences of umax $\left(\operatorname{pr}_{I^{\prime \prime}} R^{\mathcal{R}}\right), \mathbb{A}_{x_{i}}$ with respect to $\operatorname{pr}_{I^{\prime}} R^{\mathcal{R}}$ are smaller than $\mathrm{lk}_{1}, \mathrm{lk}_{2}$. Therefore the $\mathrm{Ik}_{1}$ - and $\mathrm{Ik}_{2}$-blocks $A, B$ containing $\operatorname{pr}_{I^{\prime \prime}} \mathbf{a}$ and $\mathbf{a}\left[x_{i}\right]$, respectively, are such that $Q^{\prime \prime}=\operatorname{pr}_{I^{\prime}} Q \cap(A \times B) \neq \varnothing$. Choose $\mathbf{a}^{\prime} \in \operatorname{pr}_{I^{\prime}} R^{\mathcal{R}} \cap(A \times B)$ such that $\mathbf{a} \sqsubseteq a s$ a $\mathbf{a}^{\prime}$ in $\operatorname{pr}_{I^{\prime}} R^{\mathcal{R}}$ and $\mathbf{a}^{\prime}$ is as-maximal in $Q^{\prime \prime}$. As is easily seen, such a tuple exists by the Rectangularity Corollary 34 because, since $Q^{\prime \prime}$ is linked, any $\mathbf{a}^{\prime} \in Q^{\prime \prime}$ such that $\operatorname{pr}_{I^{\prime \prime}} \mathbf{a}^{\prime}$ is as-maximal in $A$ and $\mathbf{a}^{\prime}\left[x_{i}\right]$ is as-maximal in $B$ is as-maximal in $Q^{\prime \prime}$. Now, consider

$$
\begin{aligned}
& S\left(x_{1}, \ldots, x_{i}, z_{1}, \ldots, z_{i}\right)=\exists y A\left(x_{1}, \ldots, x_{i-1}\right) \wedge B\left(x_{i}\right) \\
& \wedge \bigwedge_{j=1}^{i} R^{\mathcal{R},\left\{x_{j}, v\right\}} / \alpha\left(x_{j}, z_{j}\right) \wedge R^{\mathcal{R},\left\{v, x_{i+1}\right\}} / \alpha\left(z_{j}, y\right) \wedge R^{\mathcal{R},\left\{x_{j}, x_{i+1}\right\}}\left(x_{j}, y\right) .
\end{aligned}
$$

By the same argument as before, there is $\mathbf{c} \in S$ such that $\mathbf{a}^{\prime} \sqsubseteq a s \operatorname{pr}_{I^{\prime}} \mathbf{c}$ in $\operatorname{pr}_{I^{\prime}} S$ and $\mathbf{c}\left[z_{j}\right] \in D$ for $j \in[i]$. Since $\mathbf{a}^{\prime}$ is as-maximal in $\operatorname{pr}_{I^{\prime}} S$, we also have $\operatorname{pr}_{I^{\prime}} \mathbf{c} \sqsubseteq$ as $\mathbf{a}^{\prime}$ in $\operatorname{pr}_{I^{\prime}} S$. Therefore there is $c \in A_{\mathcal{R}, x_{i+1}}$ such that $\left(\mathbf{a}^{\prime}[x], c\right) \in Q^{x x_{i+1}}$ for every $x \in I^{\prime}$. By the 2-Decomposition Theorem 30 applied to $R^{\mathcal{R}}$, there is $\mathbf{a}^{\prime \prime} \in \operatorname{pr}_{I} R$ such that $\operatorname{pr}_{I^{\prime}} \mathbf{a}^{\prime \prime}=\mathbf{a}^{\prime}$ and $c \sqsubseteq$ as $\mathbf{a}^{\prime \prime}\left[x_{i+1}\right]$. Finally, since $\mathbf{a}$ is as-maximal in $\operatorname{pr}_{I^{\prime}} R^{\mathcal{R}}$, there is an as-path from $\mathbf{a}^{\prime}$ to $\mathbf{a}$ in $\operatorname{pr}_{I^{\prime}} R^{\mathcal{R}}$ and we complete the proof by the Maximality Lemma27(4).

Next, we consider the case $u=v$. Let $I=\left\{x_{1}, \ldots, x_{i}, v\right\}, I^{\prime}=\left\{x_{1}, \ldots, x_{i}\right\}$ and $I^{\prime \prime}=\left\{x_{1}, \ldots, x_{i-1}\right\}$. Note that by reordering the variables we may assume that if $x_{i} \in Z$ then $Z \cap\left\{x_{1}, \ldots, x_{i-1}\right\} \neq \varnothing$. By the induction hypothesis there is $c \in A_{\mathcal{R}, v}$ such that $\left(\operatorname{pr}_{I^{\prime \prime}} \mathbf{a}, c\right)$ belongs to amax $\left(\operatorname{pr}_{I^{\prime \prime} \cup\{v\}} R^{\mathcal{R}}\right)$ and is Q-compatible, in particular, $c^{\alpha} \in D$. We consider the tuple $\left(\mathbf{a}, c^{\alpha}\right)$ and relation $R^{\prime}=\operatorname{pr}_{I} R^{\mathcal{R}} / \alpha$. We have $\mathbf{a} \in \operatorname{pr}_{I^{\prime}} R^{\prime}$. For any $j \in[i-1]$ we have $(\mathbf{a}[j], c) \in Q^{x_{j} v}$ by the choice of $c$. If $x_{i} \notin Z$ then there is $c^{\prime} \stackrel{\underline{\underline{\alpha}}}{=} c$ such that $\left(\mathbf{a}\left[x_{i}\right], c^{\prime}\right) \in R^{\left\{x_{i}, v\right\}, \mathcal{R}}$. By (S3) for $\mathcal{R},\left(\mathbf{a}\left[x_{i}\right], c^{\prime}\right)$ extends to a tuple from $R^{\mathcal{R}}$, therefore $\left(\mathbf{a}\left[x_{i}\right], c\right) \in \operatorname{pr}_{x_{i} v} R^{\prime}$. If $x_{i} \in Z$, then there is also some $j \in I^{\prime \prime} \cap Z$. Then since $(\mathbf{a}[j], \mathbf{a}[i]) \in Q^{x_{j} x_{i}}$, we have $\kappa_{x_{j}}\left(\mathbf{a}\left[x_{j}\right]\right)=\kappa_{x_{i}}\left(\mathbf{a}\left[x_{i}\right]\right)=c$. Therefore there is $c^{\prime} \stackrel{\alpha}{\equiv} c$ such that $\left(\mathbf{a}\left[x_{i}\right], c^{\prime}\right) \in$ $R^{\left\{x_{i}, v\right\}, \mathcal{R}}$ and we continue as before. By the 2-Decomposition Theorem 30 there is $\mathbf{b} \in R^{\prime}$ such that $\operatorname{pr}_{I^{\prime}} \mathbf{b}=\mathbf{a}$ and $c \sqsubseteq_{a s} \mathbf{b}[v]$ in $A_{\mathcal{R}, v} / \alpha$, that is, $\mathbf{b}[v] \in D$. Therefore there is $\mathbf{c} \in \operatorname{pr}_{I} R$ such that $\operatorname{pr}_{I^{\prime}} \mathbf{c}=\mathbf{a}$ and $\mathbf{c}[v]^{\alpha} \in D$, as required.

Item (3) can be proved in the same way, as $\mathcal{P}$ is block-minimal. For (1) we need to make two changes. First, we apply the argument above for $i=2$ and stop before the last application of the 2-Decomposition Theorem 30. Second, we 
need to consider the case when extending a pair from $Q^{x y}$ by a value of $v$. More precisely, let $(a, b) \in Q^{x y}, x, y \in V$, we need to find $c \in A_{\mathcal{R}, v}$ such that $c^{\alpha} \in D$ and $(a, c) \in Q^{x v},(b, c) \in Q^{y v}$. Let

$$
Q(x, y, v)=R^{\{x, y\}, \mathcal{R}}(x, y) \wedge R^{\{x, v\}, \mathcal{R}}(x, v) \wedge R^{\{y, v\}, \mathcal{R}}(y, v) .
$$

By (2,3)-consistency and construction $(a, b) \in \operatorname{pr}_{x y} Q,\left(a, d_{1}\right) \in \operatorname{pr}_{x v} Q,\left(b, d_{2}\right) \in$ $\operatorname{pr}_{y v} Q$, where $d_{1}^{\alpha}=d_{2}^{\alpha} \in D$. The relation $Q^{\prime}=Q /{ }_{\alpha}$ then satisfies the conditions: $(a, b) \in \operatorname{pr}_{x y} Q^{\prime},(a, d) \in \operatorname{pr}_{x v} Q^{\prime},(b, d) \in \operatorname{pr}_{y v} Q^{\prime}$ where $d=d_{1}^{\alpha}=d_{2}^{\alpha}$. By the 2-Decomposition Theorem 30 there is a tuple $\left(a, b, d^{\prime}\right) \in Q^{\prime}$ such that $d \sqsubseteq$ as $d^{\prime}$, that is, $d^{\prime} \in D$. Therefore $Q$ contains a tuple $(a, b, c)$ for some $c$ with $c^{\alpha}=d^{\prime}$. This $c$ is as required.

Let $\mathcal{P}^{\prime \prime}$ be the problem obtained from $\mathcal{P}^{\prime}$ as follows: establish (2,3)-minimality and, for any $(w, \gamma, \delta) \in \mathcal{W}(\bar{\beta})$, establish minimality of $\mathcal{P}_{W_{w, \gamma \delta}}^{\prime} / \bar{\mu}^{Y}$, where $Y$ is one of the sets specified in the definition of block-minimality, until the instance does not change any longer. Let $A_{\mathcal{R}, w}^{\prime \prime}$ be the domains of $w \in V$ for $\mathcal{P}^{\prime \prime}$, for $C=\langle\mathbf{s}, R\rangle \in \mathcal{C}$ let $R^{\prime \prime}$ denote the corresponding constraint relation of $\mathcal{P}^{\prime \prime}$; for $(w, \gamma, \delta) \in \mathcal{W}(\bar{\beta})$ let $\mathcal{S}_{W_{w, \gamma \delta}, Y}^{\prime \prime}$ denote the set of solutions of $\mathcal{P}_{W_{w, \gamma \delta}}^{\prime \prime} / \bar{\mu}^{Y}$; finally $R_{C, w, \gamma \delta}^{\prime \prime}$ denote the set of tuples from $R_{C, w, \gamma \delta}$ extending to a solution of $\mathcal{P}_{W_{w, \gamma \delta}}^{\prime \prime} / \bar{\mu}^{Y}$.

The next corollary follows straightforwardly from Proposition 83 , because establishing (2,3)-minimality or minimality never eliminates an as-maximal Q-compatible tuples.

Corollary 84 The sets $A_{\mathcal{R}, w}^{\prime \prime}, R^{\prime \prime}, \mathcal{S}_{W_{w, \gamma \delta}, Y}^{\prime \prime}$, and $R_{C, w, \gamma \delta}^{\prime \prime}$ contain all the as-maximal $Q$-compatible tuples from $A_{\mathcal{R}, w}, R, \mathcal{S}_{W_{w, \gamma \delta}, Y}$, and $R_{C, w, \gamma \delta}$, respectively.

Now we can show that the collection of relations

$$
\mathcal{R}^{\prime \prime}=\left\{R_{C, w, \gamma \delta}^{\prime \prime} \mid C \in \mathcal{C},(w, \gamma, \delta) \in \mathcal{W}(\bar{\beta})\right\}
$$

is almost a $\bar{\beta}$-strategy.

Theorem 85 The collection of relations $\mathcal{R}^{\prime \prime}$ constructed as above satisfies all the conditions of a $\bar{\beta}$-strategy with respect to $\bar{B}$, except (S5) and (S6).

Proof: Condition (S1) follows from Corollary 84. Conditions (S2),(S3) follow from Corollary 84 and construction (establishing block-minimality). For (S4) every relation $R_{C, w, \gamma \delta}^{\prime \prime}$ is a subalgebra in $R_{C, w, \gamma \delta}$ and therefore in $\operatorname{pr}_{\mathbf{s} \cap W_{w, \gamma \delta}} R$ by construction. Moreover, as by Proposition $83 R_{C, w, \gamma \delta}^{\prime \prime}$ contains an element asmaximal in $R_{C, w, \gamma \delta}$, we have $\operatorname{umax}\left(R_{C, w, \gamma \delta}^{\prime \prime}\right) \subseteq \operatorname{umax}\left(R_{C, w, \gamma \delta}\right)$. Finally, condition (S7) follows from (S7) for $\mathcal{R}$. 
We will need another property of $\mathcal{P}^{\prime \prime}$. Unfortunately, Q-compatible tuples are not very helpful in establishing properties (S5),(S6), since those are properties of $\mathrm{u}$-maximal fragments of relations. Therefore we need to extend Q-compatibility to u-maximal elements. Similar to Q-compatibility we make the following definition. For $x, y \in V$, let

$$
\begin{aligned}
& P^{x}=\left\{a \in \operatorname{umax}\left(A_{\mathcal{R}, x}\right) \mid \text { there is } d \in D \text { such that }(d, a) \in R^{\{v, x\}, \mathcal{R}} / \alpha\right\}, \\
& \text { and } \\
& P^{x y}=\left\{(a, b) \in \operatorname{umax}\left(R^{\{x, y\}, \mathcal{R}}\right) \mid \text { there is } d \in D\right. \text { such that } \\
& \left.(d, a) \in R^{\{v, x\}, \mathcal{R}} / \alpha,(d, b) \in R^{\{v, y\}, \mathcal{R}} / \alpha\right\} .
\end{aligned}
$$

In particular $P^{v}=\operatorname{umax}(\widehat{D})$. Note that these relations are different from $Q^{x}, Q^{x y}$ in that they consist of u-maximal elements and pairs, rather than as-maximal as $Q^{x}, Q^{x y}$. We say that a tuple a on a set $U \subseteq V$ (where $U$ can be, e.g. a subset of $\mathbf{s}$ for a constraint $C=\langle\mathbf{s}, R\rangle$, or a subset of $W_{w, \gamma \delta}$ for some $\left.(w, \gamma, \delta) \in \mathcal{W}(\bar{\beta})\right)$ is $P$-compatible if $(\mathbf{a}[x], \mathbf{a}[y]) \in P^{x y}$ for any $x, y \in U$.

Let $\mathcal{P}^{\prime \prime \prime}$ be the instance obtained as follows: First, restrict the domains and relations of $\mathcal{P}$ to the sets of $\mathrm{P}$-compatible tuples they contain; let the new relation for $C=\langle\mathbf{s}, R\rangle \in \mathcal{C}$ be denoted by $R^{\dagger}$. Second, establish (2,3)-minimality and block-minimality of the resulting instance. Let $R^{\prime \prime \prime}, R_{C, w, \gamma \delta}^{\prime \prime \prime}, \mathcal{S}_{W_{w, \gamma \delta}, Y}^{\prime \prime \prime}$ denote the relations induced by $\mathcal{P}^{\prime \prime \prime}$. Note that the domains and relations of $\mathcal{P}^{\prime \prime \prime}$ are not necessarily subalgebras.

Lemma 86 Let $C=\langle\mathbf{s}, R\rangle \in \mathcal{C},(w, \gamma, \delta) \in \mathcal{W}(\bar{\beta})$.

(1) Relations $R^{\prime \prime \prime}, R_{C, w, \gamma \delta}^{\prime \prime \prime}, \mathcal{S}_{W_{w, \gamma \delta}, Y}^{\prime \prime}$ are nonempty, and $R^{\prime \prime \prime} \subseteq R^{\prime \prime}, R_{C, w, \gamma \delta}^{\prime \prime \prime} \subseteq$ $R_{C, w, \gamma \delta}^{\prime \prime}, \mathcal{S}_{W_{w, \gamma \delta}, Y}^{\prime \prime \prime} \subseteq \mathcal{S}_{W_{w, \gamma \delta}, Y}^{\prime \prime}$

(2) Relations $R_{C, w, \gamma \delta}^{\prime \prime \prime}$ and $\mathcal{S}_{W_{w, \gamma, \delta, \bar{\beta}}^{\prime \prime},}^{\prime \prime}$ are as-closed in $R_{C, w, \gamma \delta}$, and in $\mathcal{S}_{W_{w, \gamma, \delta}^{\prime}}^{\prime \mathcal{R}}$, respectively (recall that $\mathcal{S}_{W_{w, \gamma, \delta, \bar{\beta}}^{\prime} \mathcal{R}}$ is the set of $\mathcal{R}$-compatible tuples from $\mathcal{S}_{W_{w, \gamma, \delta, \bar{\beta}}, Y}$ ).

Proof: (1) The inclusions $R^{\prime \prime \prime} \subseteq R^{\prime \prime}, R_{C, w, \gamma \delta}^{\prime \prime \prime} \subseteq R_{C, w, \gamma \delta}^{\prime \prime}, \mathcal{S}_{W_{w, \gamma \delta, \bar{\beta}}^{\prime \prime \prime}} \subseteq$ $\mathcal{S}_{W_{w, \gamma \delta, \bar{\beta}}, Y}^{\prime \prime}$ follow from the construction, as $R^{\dagger} \subseteq R^{\prime}$. On the other hand, as every Q-compatible tuple from $R$ belongs to $R^{\dagger}$, the nonemptyness follows by Corollary 84

(2) First we observe that for any $x, y \in V$ the relation $P^{x y}$, and therefore $P^{x}$, is as-closed in $\operatorname{umax}\left(R^{\{x, y\}, \mathcal{R}}\right)$. This can be done in the same way as in the proof of Proposition 83 , Let $(a, b) \in P^{x y}$ and $\left(a^{\prime}, b^{\prime}\right) \in \operatorname{umax}\left(R^{\{x, y\}, \mathcal{R}}\right)$ be such that $(a, b) \sqsubseteq a s\left(a^{\prime}, b^{\prime}\right)$. We need to find $d \in D$ such that $\left(a^{\prime}, d\right) \in R^{\{x, v\}, \mathcal{R} / \alpha}$, 


$$
\begin{aligned}
& \left(b^{\prime}, d\right) \in R^{\{y, v\}, \mathcal{R}} / \alpha \text {. Let } \\
& \quad Q(x, y, v)=R^{\{x, y\}, \mathcal{R}}(x, y) \wedge R^{\{x, v\}, \mathcal{R}} / \alpha(x, v) \wedge R^{\{y, v\}, \mathcal{R}} / \alpha(y, v),
\end{aligned}
$$

which is a subalgebra of $\mathbb{A}_{x} \times \mathbb{A}_{y} \times \mathbb{A}_{v} / \alpha$. Since $(a, b) \in P^{x y}$ there is $c \in D$ with $(a, b, c) \in Q$. By (S1) for $\mathcal{R}$ we have $\left(a^{\prime}, b^{\prime}\right) \in \operatorname{pr}_{x y} Q$, moreover, $(a, b) \sqsubseteq a s$ $\left(a^{\prime}, b^{\prime}\right)$ in $\operatorname{pr}_{x y} Q$. By the Maximality Lemma 27(4) there is $d \in A_{\mathcal{R}, v}$ such that $\left(a^{\prime}, b^{\prime}, d^{\alpha}\right) \in Q$ and $(a, b, c) \sqsubseteq_{a s}\left(a^{\prime}, b^{\prime}, d^{\alpha}\right)$ in $Q$. Therefore $d^{\alpha} \in D$.

It suffices to prove the statement for relations of the form $R_{C, w, \gamma \delta}^{\prime \prime \prime}$, because relations $\mathcal{S}_{W_{w, \gamma, \delta}, Y}^{\prime \prime \prime}$ are pp-definable through $R_{C, w, \gamma \delta}^{\prime \prime \prime}$, and we can use Lemma 61 (2). Since $P^{x y}$ are as-closed, every relation $R_{C, w, \gamma \delta}^{\dagger}$ is also as-closed in $\operatorname{umax}\left(R_{C, w, \gamma \delta}\right)$. We prove by induction that this property is preserved as (2,3)-minimality and block-minimality is being established. The observation about the relations $R_{C, w, \gamma \delta}^{\dagger}$ establishes the base case. For the induction step, let $R_{C, w, \gamma \delta}^{\ddagger}$ and $\mathcal{S}_{W_{w, \gamma \delta}, Y}^{\ddagger}$ denote the current state of the corresponding relations, and they are as-closed. There are two cases for the induction step.

In the first case we make a step to enforce $(2,3)$-minimality, that is, for some $x, y, z \in V$ we check whether or not some $(a, b) \in R^{\ddagger\{x, y\}}$ can be extended by $c \in B_{z}$ such that $(a, c) \in R^{\ddagger\{x, z\}}$ and $(b, c) \in R^{\ddagger\{y, z\}}$. Let $(a, b) \in R^{\ddagger\{x, y\}}$ be such that there are $c \in B_{z}$ with $(a, c) \in R^{\ddagger\{x, z\}},(b, c) \in R^{\ddagger\{y, z\}}$, and let $\left(a^{\prime}, b^{\prime}\right) \in R^{\ddagger\{x, y\}}$ such that $(a, b) \sqsubseteq a s\left(a^{\prime}, b^{\prime}\right)$ in $R^{\ddagger\{x, y\}}$. Then there is $c^{\prime} \in B_{z}$ such that $\left(a^{\prime}, c^{\prime}\right) \in R^{\{x, z\}},\left(b^{\prime}, c^{\prime}\right) \in R^{\{y, z\}}$. Similar to part (1) let

$$
Q(x, y, z)=\operatorname{Sg}\left(R^{\ddagger\{x, y\}}\right)(x, y) \wedge R^{\{x, v\}, \mathcal{R}}(x, v) \wedge R^{\{y, v\}, \mathcal{R}}(y, v),
$$

We have $\operatorname{Sg}\left(R^{\ddagger\{x, y\}}\right)=\operatorname{pr}_{x y} Q$ by $(\mathrm{S} 1)$ for $\mathcal{R}$, and $(a, b, c),\left(a^{\prime}, b^{\prime}, c^{\prime}\right) \in Q$. By the Maximality Lemma 27(4) we may assume that $(a, b, c) \sqsubseteq a s\left(a^{\prime}, b^{\prime}, c^{\prime}\right)$ in $Q$. Since $R^{\ddagger\{x, z\}}, R^{\ddagger\{x, z\}}$ are as-closed, we have $\left(a^{\prime}, c^{\prime}\right) \in R^{\ddagger\{x, z\}},\left(b^{\prime}, c^{\prime}\right) \in R^{\ddagger\{x, z\}}$

In the second case we solve a subproblem of the form $\mathcal{P}_{W_{w, \gamma \delta}}^{\ddagger} / \bar{\mu}^{Y}$. Let $U=$ $W_{w, \gamma \delta}$, let $\mathcal{S}_{W_{w, \gamma \delta}, Y}^{\ddagger}$ be the corresponding set of solutions. Take $C=\langle\mathbf{s}, R\rangle \in \mathcal{C}$ and $(u, \eta, \theta) \in \mathcal{W}(\bar{\beta})$, let $U^{\prime}=\mathbf{s} \cap U \cap W_{u, \eta \theta}$. Suppose a $\in R_{C, u, \eta \theta}^{\ddagger}$ be such that there is a solution $\varphi \in \mathcal{S}_{W_{w, \gamma \delta, \bar{\beta}}, Y}^{\ddagger}$ extending $\operatorname{pr}_{U^{\prime}} \mathbf{a}$, and let $\mathbf{b} \in R_{C, u, \eta \theta}^{\ddagger}$ is such that $\mathbf{a} \sqsubseteq a s$ b in $R_{C, u, \eta \theta}^{\ddagger}$. We need to show that $\mathrm{pr}_{U^{\prime}} \mathbf{b}$ is extendible to a solution from $\mathcal{S}_{W_{w, \gamma \delta}, Y}^{\ddagger}$. Let $\psi \in \mathcal{S}_{W_{w, \gamma \delta}, Y}^{\prime \mathcal{R}}$ be a solution extending $\operatorname{pr}_{U^{\prime}} \mathbf{b}$. Since $\operatorname{pr}_{U^{\prime}} \mathbf{a} \sqsubseteq a s \operatorname{pr}_{U^{\prime}} \mathbf{b}$ by the Maximality Lemma 27(4) we may assume that $\varphi \sqsubseteq_{a s} \psi$ in $\mathcal{S}_{W_{w, \gamma \delta, \bar{\beta}}, Y}^{\prime \mathcal{R}}$. Since by the induction hypothesis $\mathcal{S}_{W_{w, \gamma \delta, \bar{\beta}}, Y}^{\ddagger}$ is as-closed, the result follows. 


\subsubsection{Step 2.}

In this step we tighten the 'near-strategy' $\mathcal{R}$ " in a way similar to that from Section 10.1. We start with showing that the domains of all variables in $W_{v, \alpha \beta}$ have to be tightened.

Lemma 87 For every $w \in W=W_{v, \alpha \beta_{v}}$ there is a congruence $\alpha_{w} \in \operatorname{Con}\left(\mathbb{A}_{w}\right)$ with $\alpha_{w} \prec \beta_{w}$, and such that $\mathcal{S}_{v w} \cap\left(B_{v} \times B_{w}\right)$ is aligned with respect to $\left(\alpha, \alpha_{w}\right)$, that is, for any $\left(a_{1}, a_{2}\right),\left(b_{1}, b_{2}\right) \in \mathcal{S}_{v w} \cap\left(B_{v} \times B_{w}\right), a_{1} \stackrel{\alpha}{\equiv} b_{1}$ if and only if $a_{2} \stackrel{\alpha_{w}}{=} b_{2}$.

Proof: It suffices to show that the link congruences $\mathrm{lk}_{1}, \mathrm{Ik}_{2}$ of $Q=\mathcal{S}_{v w}$ viewed as a subdirect product of $\mathbb{A}_{v} \times \mathbb{A}_{w}$ are such that $\beta_{v} \wedge \mathrm{Ik}_{1} \leq \alpha$ and $\beta_{w} \wedge \mathrm{Ik}_{2}<\beta_{w}$. Since $w \in W$ there are $\gamma, \delta \in \operatorname{Con}\left(\mathbb{A}_{w}\right)$ such that $\gamma \prec \delta \leq \beta_{w}$ and $\left(\alpha, \beta_{v}\right)$ and $(\gamma, \delta)$ cannot be separated. By Lemma 49 it follows that $\beta_{v} \wedge \mathrm{Ik}_{1} \leq \alpha$ and $\mathrm{Ik}_{2} \wedge \delta \leq \gamma$. We set $\alpha_{w}=\beta_{w} \wedge \mathrm{Ik}_{2}<\beta_{w}$. Since $B_{w} / \alpha_{w}$ is isomorphic to $B_{v} / \alpha$, $\alpha_{w} \prec \beta_{w}$.

Let $\beta_{v}^{\prime}=\alpha, \beta_{w}^{\prime}=\alpha_{w}$ for $w \in W=W_{v, \alpha \beta_{v}}$, and $\beta_{w}^{\prime}=\beta_{w}$ for $w \in$ $V-W$. Lemma 87 implies that there is an isomorphism $\nu_{w}: B_{v} / \beta_{v}^{\prime} \rightarrow B_{w} / \beta_{w}^{\prime}$. Choose an as-maximal $\beta_{v}^{\prime}$-block $B$, an element of $D$ from Step 1 and set $B_{v}^{\prime}=B$, $B_{w}^{\prime}=\nu_{w}(B)$ for $w \in V-W$, and $B_{w}^{\prime}=B_{w}$ for $w \in V-W$. Let $\mathcal{P}^{*}$ be the problem instance obtained from $\mathcal{P}^{\prime \prime}$ as follows: first restrict the domain of $w \in W$ in $\mathcal{P}^{\prime \prime}$ to $B_{w}^{\prime}$, then establish the (2,3)-minimality of the resulting problem, and finally, establish the minimality of all problems of the form $\mathcal{P}_{W_{w, \gamma \delta}}^{\prime \prime} / \bar{\mu}^{Y}$ for $(w, \gamma, \delta) \in \mathcal{W}(\bar{\beta})$, where $Y$ is a set specified in the definition of block-minimality for $\mathcal{P}$.

Let $\mathcal{R}^{*}$ be the following collection of relations;

(T1) $\mathcal{R}^{*}=\left\{R_{C, w, \gamma \delta}^{*} \mid C=\langle\mathbf{s}, R\rangle \in \mathcal{C},(w, \gamma, \delta) \in \mathcal{W}\left(\bar{\beta}^{\prime}\right)\right\}$;

(T2) for every $C=\langle\mathbf{s}, R\rangle \in \mathcal{C},(u, \gamma, \delta) \in \mathcal{W}\left(\bar{\beta}^{\prime}\right), R_{C, u, \gamma \delta}^{*}=\operatorname{pr}_{\mathbf{s} \cap W_{u, \gamma \delta}} R^{*}$, where $R^{*}$ is the constraint relation of $\mathcal{P}^{*}$ obtained from $R$.

Lemma 88 (1) For every constraint $C=\langle\mathbf{s}, R\rangle \in \mathcal{C}$, and every a $\in \operatorname{umax}\left(R^{\prime \prime}\right)$ such that $\mathbf{a}[u] \in \nu_{u}(D)$ for $u \in \mathbf{s} \cap W$ there is a tuple $\mathbf{b} \in \operatorname{umax}\left(R^{\prime \prime}\right)$ such that $\operatorname{pr}_{\mathbf{s}-W} \mathbf{b}=\operatorname{pr}_{\mathbf{s}-W} \mathbf{a}$ and $\mathbf{b}[u] \in B_{u}^{\prime}$ for $u \in \mathbf{s} \cap W$.

(2) Let $C=\langle\mathbf{s}, R\rangle \in \mathcal{C},(w, \gamma, \delta) \in \mathcal{W}\left(\bar{\beta}^{\prime}\right), W^{\prime}=W_{w, \gamma \delta}$, and $\mathbf{a} \in \operatorname{umax}\left(R_{C, w, \gamma \delta}^{\prime \prime}\right)$ such that $\mathbf{a}[u] \in \nu_{u}(D)$ for $u \in \mathbf{s} \cap W^{\prime} \cap W$. Then there is $\left.\mathbf{b} \in \operatorname{umax}\left(R_{C, w, \gamma \delta}^{\prime \prime}\right)\right)$ such that $\operatorname{pr}_{\left(\mathbf{s} \cap W^{\prime}\right)-W} \mathbf{b}=\operatorname{pr}_{\left(\mathbf{s} \cap W^{\prime}\right)-W} \mathbf{a}$ and $\mathbf{b}[u] \in B_{u}^{\prime}$ for $u \in \mathbf{s} \cap W^{\prime} \cap W$.

(3) Let $(w, \gamma, \delta) \in \mathcal{W}\left(\bar{\beta}^{\prime}\right)$ and $W^{\prime}=W_{w, \gamma \delta}$. Let $\mathcal{S}_{W^{\prime}}^{\prime \prime}$ be the set of solutions of $\mathcal{P}_{W^{\prime}}^{\prime \prime} / \bar{\mu}^{Y}$, where $Y=\varnothing$ if $(w, \gamma, \delta) \notin \mathcal{W}^{\prime}$, and is one of the sets specified in the 
definition of block-minimality otherwise. For every solution $\varphi \in \operatorname{umax}\left(\mathcal{S}_{W^{\prime}}^{\prime \prime}\right)$ such that $\varphi[u] \in \nu_{u}(D)$ for $u \in W^{\prime} \cap W$ there is a solution $\psi \in \operatorname{umax}\left(\mathcal{S}_{W^{\prime}}^{\prime \prime}\right)$ such that $\psi(u)=\varphi(u)$ for $u \in W^{\prime}-W$ and $\psi(u) \in B^{\prime}(u)$ for $u \in W^{\prime} \cap W$.

Proof: (1) Let $U_{1}=\mathbf{s} \cap W$ and $U_{2}=\mathbf{s}-W$. If $U_{1}=\varnothing$ there is nothing to prove; assume $U_{1} \neq \varnothing$. It suffices to consider $Q=R^{\prime \prime} / \bar{\alpha}^{\prime}$ where $\alpha_{u}^{\prime}=\beta_{u}^{\prime}$ if $u \in U_{1}$ and $\alpha_{u}^{\prime}=\underline{0}_{u}$ otherwise. So, we assume $\beta_{u}^{\prime}=\underline{0}_{u}$ for all $u \in U_{1}$. Then for any $u_{1}, u_{2} \in U_{1}$ and any $\mathbf{d} \in R$ such that $\mathbf{d}\left[u_{1}\right] \in B_{u_{1}}, \mathbf{d}\left[u_{2}\right] \in B_{u_{2}}$ we have $\mathbf{d}\left[u_{2}\right]=\nu_{u_{2}} \circ \nu_{u_{1}}^{-1}\left(\mathbf{d}\left[u_{1}\right]\right)$. Therefore we may assume that $\left|U_{1}\right|=1$, say, $U_{1}=\{u\}$.

Considering $R^{\prime \prime}$ as a subalgebra of $\mathbb{A}_{u} \times \operatorname{pr}_{\mathbf{s}-\{u\}} R$, the result follows by the Congruence Lemma64. Indeed, since there is a $\alpha_{u} \beta_{u}$-collapsing polynomial $f$ of $R$, that is, $f\left(\bar{\beta}_{\mathbf{s}-\{u\}}\right) \subseteq \underline{0}_{\mathbf{s}-\{u\}}$, there are no $\eta, \theta \in \operatorname{Con}\left(\operatorname{pr}_{\mathbf{s}-\{u\}} R\right)$ with $\eta \prec \theta \leq$ $\bar{\beta}_{\mathbf{s}-\{u\}}$ such that $\left(\alpha_{u}, \beta_{u}\right)$ cannot be separated from $(\eta, \theta)$.

(2) and (3) are proved in essentially the same way.

To show that $\mathcal{P}^{*}$ has the desirable properties, in particular, it is nonempty, we consider a collection of unary and binary relations similar to $Q^{x}, Q^{x y}$ from Step 1 . For $x, y \in V$ let $T^{x}, T^{x y}$ denote the following sets:

$$
\begin{aligned}
T^{x}= & \left\{a \in \operatorname{amax}\left(A_{\mathcal{R}, x}^{\prime \prime}\right) \mid(a, c) \in \operatorname{amax}\left(R^{\prime \prime\{x, v\}, \mathcal{R}}\right) \text { for some } c \in B\right\} ; \\
T^{x y}= & \left\{(a, b) \in \operatorname{amax}\left(R^{\prime \prime\{x, y\}, \mathcal{R}}\right) \mid(a, c) \in \operatorname{amax}\left(R^{\prime \prime\{x, v\}, \mathcal{R}}\right),\right. \\
(b, c) \in \operatorname{amax}\left(R^{\prime \prime}\{y, v\}, \mathcal{R}\right. & \text { for some } c \in B\} .
\end{aligned}
$$

A tuple a over a set of variables $U \subseteq V$ is said to be T-compatible if for any $x, y \in U,(\mathbf{a}[x], \mathbf{a}[y]) \in T^{x y}$. The following lemma provides the main structural result necessary for proving that $\mathcal{R}^{*}$ is a $\bar{\beta}^{*}$-strategy.

Lemma 89 Let $S$ be one of the relations $R, R_{C, w, \gamma \delta}, \mathcal{S}_{W_{w, \gamma, \delta}, Y}$, and $S^{\prime \prime}, S^{*}$ the corresponding relations $R^{\prime \prime}, R_{C, w, \gamma \delta}^{\prime \prime}$, or $\mathcal{S}_{W_{w, \gamma, \delta}, Y}^{\prime \prime}$, and $R^{*}, R_{C, w, \gamma \delta}^{*}$, or $\mathcal{S}_{W_{w, \gamma, \delta}, Y}^{*}$, respectively, for some $C=\langle\mathbf{s}, R\rangle \in \mathcal{C}$ and $(w, \gamma, \delta) \in \mathcal{W}(\bar{\beta})$, where $Y$ is a set from the definition of block-minimality; and let $U$ be its set of coordinate positions.

(1) Every $Q$-compatible $\mathbf{a} \in \operatorname{amax}(S)$ such that $\mathbf{a}[u] \in B_{u}^{\prime}$ for $u \in U \cap W$ is also T-compatible.

(2) $S^{*}$ contains all the as-maximal T-compatible tuples from $S^{\prime \prime}$.

(3) If $S^{*}=R_{C, w, \gamma \delta}^{*}$, then $\operatorname{umax}\left(S^{*}\right)$ is as-closed in $\operatorname{umax}\left(R_{C, w, \gamma \delta} \cap \bar{B}^{\prime}\right)$.

Proof: (1) By Corollary 84, if, say $x \in W$ and $(a, b) \in Q^{x y}$ are such that $a \in$ $B_{x}^{\prime}$, then $(a, b) \in T^{x y}$, which can be proved as in the proof of Proposition 83(1). Therefore, it suffices to prove that if $x, y \notin W$, then $T^{x y}=Q^{x y}$. This can be done 
in the same way as in the proof of Proposition 83, Let $(a, b) \in Q^{x y}$, we need to find $c \in B_{v}^{\prime}$ such that $(a, c) \in Q^{x v},(b, c) \in Q^{y v}$. Let

$$
Q(x, y, v)=R^{\{x, y\}, \mathcal{R}}(x, y) \wedge R^{\{x, v\}, \mathcal{R}}(x, v) \wedge R^{\{y, v\}, \mathcal{R}}(y, v),
$$

which is a subalgebra of $\mathbb{A}_{x} \times \mathbb{A}_{y} \times \mathbb{A}_{v}$. By $(2,3)$-consistency of $\mathcal{P}^{\prime \prime} Q^{x y} \subseteq$ $\operatorname{pr}_{x y} Q, Q^{x v} \subseteq \operatorname{pr}_{x v} Q, Q^{y v} \subseteq \operatorname{pr}_{x v} Q$. Let $Q^{\prime}=Q / \alpha$. Since each of $R^{\{x, y\}, \mathcal{R}}$, $R^{\{x, v\}, \mathcal{R}}, R^{\{y, v\}, \mathcal{R}}$ is polynomially closed in the corresponding constraint relation $R^{\{x, y\}}, R^{\{x, v\}}$, or $R^{\{y, v\}}$ of $\mathcal{P}, Q$ is polynomially closed in

$$
R^{\{x, y\}}(x, y) \wedge R^{\{x, v\}}(x, v) \wedge R^{\{y, v\}}(y, v),
$$

as well, and so is $Q^{\prime}$ in

$$
R^{\{x, y\}}(x, y) \wedge R^{\{x, v\}} / \alpha(x, v) \wedge R^{\{y, v\}} / \alpha(y, v) .
$$

Let $Q^{\dagger}=\operatorname{pr}_{x y}\left(Q^{\prime} \cap\left(B_{x} \times B_{y} \times D\right)\right)$, By the Congruence Lemma 64 either umax $\left(Q^{\dagger}\right) \times D \subseteq Q^{\prime}$ or there is $\eta \in \operatorname{Con}\left(R^{\{x, y\}}\right)$ such that umax $\left(Q^{\prime} \cap\left(B_{x} \times\right.\right.$ $\left.B_{y} \times D\right)$ ) is the graph of a mapping $\tau: \operatorname{umax}\left(Q^{\dagger}\right) \rightarrow D$. In the former case we are done, because then $\left(a, b, B_{v}^{\prime}\right) \in Q^{\prime}$, and therefore $(a, b, c) \in Q$ for some $c \in B_{v}^{\prime}$. The latter case is not possible, because by the Congruence Lemma 64 there is $\theta \in \operatorname{Con}\left(R^{\{x, y\}}\right)$ such that $\eta \prec \theta \leq \beta_{x} \times \beta_{y}$, and $\left(\alpha, \beta_{v}\right)$ and $(\eta, \theta)$ cannot be separated. This however is not the case, since $x, y \notin W$.

(2) Similar to Proposition 83 and Corollary 84 it suffices to prove that for any $X \subseteq U$, any T-compatible a $\in \operatorname{amax}\left(\operatorname{pr}_{X} S^{\prime \prime}\right)$ can be extended to a T-compatible $\mathbf{b} \in \operatorname{amax}\left(S^{\prime \prime}\right)$. In fact, since $S^{\prime \prime}$ contains all the Q-compatible tuples, and therefore all the T-compatible tuples from $S$, it suffices to prove the statement for $S$, rather than for $S^{\prime \prime}$. We show that for any $w \in U-X$ tuple a can be extended to a T-compatible tuple $\mathbf{c} \in \operatorname{amax}\left(\operatorname{pr}_{X \cup\{w\}} S\right)$. By Proposition 83 there is a Qcompatible $\mathbf{b}^{\prime} \in S$ with $\mathbf{a}=\operatorname{pr}_{X} \mathbf{b}^{\prime}$. If $X \cap W \neq \varnothing$ or $(X \cup\{w\}) \cap W=\varnothing$, we can set $\mathbf{c}=\operatorname{pr}_{X \cup\{w\}} \mathbf{b}^{\prime}$.

Suppose that $X \cap W=\varnothing$ and $w \in W$. Then we proceed similar to part (1). Let $X=\left\{x_{1}, \ldots, x_{k}\right\}$, let

$$
Q\left(x_{1}, \ldots, x_{k}, w\right)=\operatorname{pr}_{X} S\left(x_{1}, \ldots, x_{k}\right) \wedge \bigwedge_{i=1}^{k} R^{\left\{x_{i}, w\right\}, \mathcal{R}}\left(x_{i}, w\right),
$$

By (2,3)-consistency of $\mathcal{P}^{\prime \prime}$ and Proposition $83 Q^{x_{i} w} \subseteq \operatorname{pr}_{x_{i} w} Q$, and by Corollary $84 S^{\prime} \subseteq \operatorname{pr}_{X} Q$, where $S^{\prime}$ is the set of all T-compatible (equivalently, Qcompatible) tuples from $\operatorname{pr}_{X} S$. Let $Q^{\prime}=Q / \alpha$. Since each of $R^{\left\{x_{i}, w\right\}, \mathcal{R}}$ is polynomially closed in $R^{\left\{x_{i}, w\right\}}$ and $\operatorname{pr}_{X} S\left(x_{1}, \ldots, x_{k}\right)$ is polynomially closed in itself, 
by Lemma 61(2) $Q$ is polynomially closed in

$$
\operatorname{pr}_{X} S\left(x_{1}, \ldots, x_{k}\right) \wedge \bigwedge_{i=1}^{k} R^{\left\{x_{i}, w\right\}}\left(x_{i}, w\right),
$$

as well, and so is $Q^{\prime}$ in

$$
\operatorname{pr}_{X} S\left(x_{1}, \ldots, x_{k}\right) \wedge \bigwedge_{i=1}^{k} R^{\left\{x_{i}, w\right\}} / \alpha_{w}\left(x_{i}, w\right) .
$$

Let $Q^{\dagger}=\operatorname{pr}_{X}\left(Q^{\prime} \cap\left(\prod_{i=1}^{k} B_{x_{i}} \times D\right)\right)$. Now we can finish the proof in the same way as in part (1).

(3) Let $U=\mathbf{s} \cap W_{w, \delta \gamma}$. Observe first, that every tuple from $\operatorname{umax}\left(R_{C, w, \gamma \delta}^{*}\right)$ is P-compatible (see Section 10.2.1). If we prove that $R_{C, w, \gamma \delta}^{*}$ contains every Pcompatible tuple a from $R_{C, w, \gamma \delta}^{\prime \prime}$ such that $\mathbf{a}[u] \in B_{u}^{\prime}$ for every $u \in U \cap W$, by Lemma 86 (2) the result follows. As in the proof of Lemma 86 we proceed by induction on the restriction of the problem $\mathcal{P}^{\prime \prime}$ being converted to a $(2,3)$-minimal and block-minimal instance.

Let $R^{\prime \prime}$ and $R_{C, w, \gamma \delta}^{\prime \prime}$ denote the relations associated with the instance $\mathcal{P}^{\prime \prime}$. Let $R^{\dagger}$ and $R_{C, w, \gamma \delta}^{\dagger}$ denote the relations obtained from $R^{\prime \prime}$ and $R_{C, w, \gamma \delta}^{\prime \prime}$ in the first step of converting $\mathcal{P}^{\prime \prime}$ to $\mathcal{P}^{*}$, that is, restricting the domains. By Lemma 88 relations $R^{\dagger}, R_{C, w, \gamma \delta}^{\dagger}$ contain all the necessary P-compatible relations. This provides the base case. For the induction step we again consider two cases. We denote the current constraint relations by $R_{C, w, \gamma \delta}^{\ddagger}$ and the ones from the (2,3)-strategy by $R^{\ddagger X}$.

In the first case we enforce (2,3)-minimality for $x, y, z \in V$. Let $(a, b) \in$ $R^{\ddagger\{x, y\}}$ be a P-compatible tuple. Then there is $c_{1}, c_{2} \in B_{z}$ such that $\left(a, c_{1}\right) \in$ $R^{\prime \prime\{x, z\}},\left(b, c_{2}\right) \in R^{\prime \prime\{y, z\}}$ are P-compatible. As in the proof of item (1) of this lemma, we can argue that $c_{1}=c_{2}$ can be assumed. If $z \notin W$, the pairs $\left(a, c_{1}\right),\left(b, c_{2}\right)$ are as required. Otherwise, $c_{1}, c_{2}$ can be chosen from $B_{z}^{\prime}$ by Lemma 88 .

In the second case let $(u, \eta, \theta) \in \mathcal{W}(\bar{\beta})$ and $X=W_{u, \eta \theta}$; we solve a problem of the form $\mathcal{P}_{X}^{\prime \prime} / \bar{\mu}^{Y}$, let $\mathcal{S}_{X}^{\ddagger}$ be the set of solutions of this problem. Let also $U^{\prime}=$ $\mathbf{s} \cap X$. We need to show that for any P-compatible $\mathbf{a} \in \operatorname{umax}\left(R_{C, w, \gamma \delta}^{\ddagger}\right)$ with $\mathbf{a}[u] \in B_{u}^{\prime}$ for $u \in U$ the tuple $\operatorname{pr}_{U^{\prime}} \mathbf{a}$ can be extended to a P-compatible solution $\varphi \in \mathcal{S}_{X}^{\ddagger}$. Since $\mathbf{a} \in \operatorname{umax}\left(R_{C, w, \gamma \delta}^{\prime \prime}\right)$, the tuple $\operatorname{pr}_{U^{\prime}} \mathbf{a}$ can be extended to a umaximal solution $\varphi \in \mathcal{S}_{X}^{\prime \prime}$. If $U^{\prime} \cap W \neq \varnothing$ or $X \cap W=\varnothing$, solution $\varphi$ is as required. Otherwise by Lemma $88 \varphi$ can be chosen P-compatible and such that $\varphi(x) \in B_{x}^{\prime}$ for $x \in X \cap W$; that is $\varphi \in \mathcal{S}_{X}^{\ddagger}$ by the induction hypothesis.

Now we are ready to prove that $\mathcal{R}^{*}$ is a $\bar{\beta}^{\prime}$-strategy. 
Theorem $90 \mathcal{R}^{*}$ is a $\bar{\beta}^{\prime}$-strategy with respect to $\bar{B}^{\prime}$.

Proof: (S1) follows directly from the construction, since the relations $R^{X, \mathcal{R}^{*}}$ result from establishing (2,3)-minimality of $\mathcal{P}^{*}$, and they are nonempty by Lemma 89 (1). Conditions (S2) and (S3) are also by construction. Condition (S4) also holds by construction, as all the relations of the form $R_{C, w, \gamma \delta}^{*}$ are subalgebras. Also, each of them contains a Q-compatible element, which is as-maximal in $R_{C, w, \gamma \delta}$, implying that $\operatorname{umax}\left(R_{C, w, \gamma \delta}^{*}\right) \subseteq \operatorname{umax}\left(R_{C, w, \gamma \delta}\right)$.

For (S5) the existence of $A_{\mathcal{R}^{*}, w}$ for $w \in V$ follows from the construction, and the as-closeness of umax $\left(A_{\mathcal{R}^{*}, w}\right)$ follows from Lemma 89. (3). Condition (S6) follows from Lemma 89 (3) and (S6) for $\mathcal{R}$ as well. Finally, condition (S7) holds by Lemma 65 ,

\section{References}

[1] Eric Allender, Michael Bauland, Neil Immerman, Henning Schnoor, and Heribert Vollmer. The complexity of satisfiability problems: Refining Schaefer's theorem. In MFCS, pages 71-82, 2005.

[2] Libor Barto. The dichotomy for conservative constraint satisfaction problems revisited. In LICS, pages 301-310, 2011.

[3] Libor Barto. The constraint satisfaction problem and universal algebra. The Bulletin of Symbolic Logic, 21(3):319-337, 2015.

[4] Libor Barto. The collapse of the bounded width hierarchy. J. Log. Comput., 26(3):923-943, 2016.

[5] Libor Barto and Marcin Kozik. Absorbing subalgebras, cyclic terms, and the constraint satisfaction problem. Logical Methods in Computer Science, 8(1), 2012.

[6] Libor Barto and Marcin Kozik. Constraint satisfaction problems solvable by local consistency methods. J. ACM, 61(1):3:1-3:19, 2014.

[7] Libor Barto, Marcin Kozik, and Todd Niven. The CSP dichotomy holds for digraphs with no sources and no sinks (A positive answer to a conjecture of Bang-Jensen and Hell). SIAM J. Comput., 38(5):1782-1802, 2009.

[8] Libor Barto, Marcin Kozik, and Ross Willard. Near unanimity constraints have bounded pathwidth duality. In LICS, pages 125-134, 2012. 
[9] Libor Barto, Andrei A. Krokhin., and Ross Willard. Polymorphisms, and How to Use Them. In The Constraint Satisfaction Problem: Complexity and Approximability [Result of a Dagstuhl Seminar], pages 1-44, 2017.

[10] Libor Barto, and Marcin Kozik. Polymorphisms, and How to Use Them. In The Constraint Satisfaction Problem: Complexity and Approximability [Result of a Dagstuhl Seminar], pages 45-77, 2017.

[11] Joel Berman, Pawel Idziak, Petar Marković, Ralph McKenzie, Matthew Valeriote, and Ross Willard. Varieties with few subalgebras of powers. Trans. Amer. Math. Soc., 362(3):1445-1473, 2010.

[12] Jonah Brown-Cohen and Prasad Raghavendra. Correlation Decay and Tractability of CSPs. In ICALP, pages 79:1-79:13, 2016.

[13] Andrei A. Bulatov. Three-element Mal'tsev algebras. Acta Sci. Math (Szeged), 71(3-4):469-500, 2002.

[14] Andrei A. Bulatov. A dichotomy theorem for constraints on a three-element set. In FOCS, pages 649-658, 2002.

[15] Andrei A. Bulatov. Tractable conservative constraint satisfaction problems. In LICS, pages 321-330, 2003.

[16] Andrei A. Bulatov. A graph of a relational structure and constraint satisfaction problems. In LICS, pages 448-457, 2004.

[17] Andrei A. Bulatov. A dichotomy theorem for constraint satisfaction problems on a 3-element set. J. ACM, 53(1):66-120, 2006.

[18] Andrei A. Bulatov. Complexity of conservative constraint satisfaction problems. ACM Trans. Comput. Log., 12(4):24, 2011.

[19] Andrei A. Bulatov. Conservative constraint satisfaction re-revisited. Journal of Computer and System Sciences, 82(2):347-356, 2016.

[20] Andrei A. Bulatov. Graphs of finite algebras, edges, and connectivity. CoRR abs/1601.07403 (2016).

[21] Andrei A. Bulatov. Graphs of relational structures: restricted types. In LICS, pages 642-651, 2016.

[22] Andrei A. Bulatov. Constraint satisfaction problems over semilattice block Mal'tsev algebras. CoRR, abs/1701.02623, 2017 (accepted to LICS 2017). 
[23] Andrei A. Bulatov. A dichotomy theorem for nonuniform CSPs. CoRR, abs/1703.03021, 2017.

[24] Andrei A. Bulatov and Víctor Dalmau. A simple algorithm for Mal'tsev constraints. SIAM J. Comput., 36(1):16-27, 2006.

[25] Andrei A. Bulatov and Peter Jeavons. Algebraic structures in combinatorial problems. Technische universität Dresden, MATH-AL-4-2001, 2001

[26] Andrei A. Bulatov, Peter Jeavons, and Andrei A. Krokhin. Classifying the complexity of constraints using finite algebras. SIAM J. Comput., 34(3):720$742,2005$.

[27] Andrei A. Bulatov, Andrei A. Krokhin, and Benoit Larose. Dualities for constraint satisfaction problems. In Complexity of Constraints - An Overview of Current Research Themes [Result of a Dagstuhl Seminar], pages 93-124, 2008.

[28] Andrei A. Bulatov and Matthew Valeriote. Recent results on the algebraic approach to the CSP. In Complexity of Constraints - An Overview of Current Research Themes [Result of a Dagstuhl Seminar]., pages 68-92, 2008.

[29] S. Burris and H.P. Sankappanavar. A course in universal algebra, volume 78 of Graduate Texts in Mathematics. Springer-Verlag, New York-Berlin, 1981.

[30] Hubie Chen and Benoit Larose. Asking the metaquestions in constraint tractability. CoRR, abs/1604.00932, 2016.

[31] Víctor Dalmau and Andrei A. Krokhin. Majority constraints have bounded pathwidth duality. Eur. J. Comb., 29(4):821-837, 2008.

[32] Rina Dechter. Constraint processing. Morgan Kaufmann Publishers, 2003.

[33] Tomas Feder and Moshe Y. Vardi. Monotone monadic SNP and constraint satisfaction. In STOC, pages 612-622, 1993.

[34] Tomas Feder and Moshe Y. Vardi. The computational structure of monotone monadic SNP and constraint satisfaction: A study through datalog and group theory. SIAM Journal of Computing, 28:57-104, 1998.

[35] Ralph Freese and Matthew Valeriote. On the Complexity of Some Maltsev Conditions. IJAC, 19(1):41-77, 2009. 
[36] Georg Gottlob, Gianluigi Greco, and Francesco Scarcello. Treewidth and Hypertree Width. In Tractability: Practical Approaches to Hard Problems, Cambridge University Press, pages 3-38, 2014.

[37] Pavol Hell and Jaroslav Nešetřil. On the complexity of $H$-coloring. Journal of Combinatorial Theory, Ser.B, 48:92-110, 1990.

[38] Pavol Hell and Jaroslav Nešetřil. Graphs and homomorphisms, volume 28 of Oxford Lecture Series in Mathematics and its Applications. Oxford University Press, 2004.

[39] David Hobby and Ralph N. McKenzie. The Structure of Finite Algebras, volume 76 of Contemporary Mathematics. American Mathematical Society, Providence, R.I., 1988.

[40] Pawel M. Idziak, Petar Markovic, Ralph McKenzie, Matthew Valeriote, and Ross Willard. Tractability and learnability arising from algebras with few subpowers. SIAM J. Comput., 39(7):3023-3037, 2010.

[41] Peter G. Jeavons, David A. Cohen, and Marc Gyssens. Closure properties of constraints. J. ACM, 44(4):527-548, 1997.

[42] Peter G. Jeavons. On the algebraic structure of combinatorial problems. Theoretical Computer Science, 200:185-204, 1998.

[43] Peter G. Jeavons, David A. Cohen, and Martin C. Cooper. Constraints, consistency and closure. Artificial Intelligence, 101(1-2):251-265, 1998.

[44] Keith Kearnes. Idempotent simple algebras. In Logic and algebra (Pontignano, 1994), Lecture Notes in Pure and Appl. Math. 180, pages 529-572, 1996.

[45] Phokion Kolaitis and Moshe Vardi. A game-theoretic approach to constraint satisfaction. In $A A A I$, pages 175-181, 2000.

[46] Phokion Kolaitis. Constraint satisfaction, databases, and logic. In IJCAI, pages 1587-1595, 2003.

[47] Marcin Kozik. Weak consistency notions for all the CSPs of bounded width. In LICS, pages 633-641, 2016.

[48] Andrei A. Krokhin and Stanislav Zivny (eds.). The Constraint Satisfaction Problem: Complexity and Approximability. Dagstuhl Follow-Ups, vol. 7, Schloss Dagstuhl - Leibniz-Zentrum fuer Informatik, 2017. 
[49] Gábor Kun and Mario Szegedy. A new line of attack on the dichotomy conjecture. Eur. J. Comb., 52:338-367, 2016.

[50] Benoit Larose, Cynthia Loten, and Claude Tardif. A characterisation of firstorder constraint satisfaction problems. Logical Methods in Computer Science, 3(4), 2007.

[51] Petar Marković. The complexity of CSPs on a 4-element set. Personal communication, 2011.

[52] Miklós Maróti. Malcev on top. Manuscript, available at http://www.math.u-szeged.hu/ nmaroti/pdf / 200x\%20Malt sev\% $200 n \div 20$ top.pdf, 2011.

[53] Miklós Maróti. Tree on top of Malcev. Manuscript, available at http://www.math.u-szeged.hu/ /nmaroti/pdf/200x\%20Tree\% $200 n \div 20$ top $\% 200$ f 20 Maltsev.pdf, 2011.

[54] Miklós Maróti and Ralph McKenzie. Existence theorems for weakly symmetric operations. Algebra universalis, 59(3), pages 463-489, 2008.

[55] Ralph N. McKenzie, George McNulty, and Walter Taylor. Algebras, Lattices, Varieties, I. Wadsworth-Brooks/Cole, Monterey, California, 1987.

[56] Ian Payne. A CSP algorithm for some subvarieties of Maltsev products. Oral communication, 2016.

[57] Thomas J. Schaefer. The complexity of satisfiability problems. In STOC, pages 216-226, 1978.

[58] Dmitriy Zhuk. On key relations preserved by a weak near-unanimity function. In ISMVL, pages 61-66, 2014.

[59] Dmitriy Zhuk. On CSP dichotomy conjecture. In Arbeitstagung Allgemeine Algebra AAA'92, page 32, 2016.

[60] Dmitriy Zhuk. The proof of CSP dichotomy conjecture for 5-element domain. In Arbeitstagung Allgemeine Algebra AAA'91, 2016. 


\section{Index}

$(\gamma, \delta, \bar{B})$-good polynomial, 37

$\alpha$-maximal element, 34

$\alpha \beta$-minimal element, 49

$\alpha_{i} \alpha_{j}$-aligned coordinates, 12,50

$\mathcal{R}$-compatible tuple, 9, 21, 67

$\mathcal{U}$-chained congruences, 36

$\bar{B}$-preserving, 37

$\bar{\alpha}$-aligned instance, 51

$\bar{\beta}$-strategy, 67

(2,3)-consistent instance, 9, 21

(2,3)-minimal instance, 9, 21

(2,3)-strategy, 9, 21

1-minimal instance, 9, 21

as-closed set, 51

as-components, 27

as-maximal, 27

as-path, 27

asm-path, 27

block-minimal instance, 14, 62

body, 22

bounded width, 9

cannot be separated, 43

chained, 37

collapsing polynomial, 44

consistent mappings, 65

constraint relation, 6, 20

Constraint Satisfaction Problem, 5, 20

constraint scope, 6, 20

constraints, 5,20

domain, 8,20

edge, 23

extension of a polynomial, 36

full congruence, 70 globally minimal instance, 9, 20

idempotent algebra, 7

instance, 5, 20

invariant relation, 6

link congruences, 31

link tolerances, 31

linked relation, 31

majority type, 23

maximal components, 25

maximal congruence, 70

maximal element, 25

minimal instance, 9, 20

minimal set, 21

module, 23

monolith, 13

nonuniform Constraint Satisfaction Problem, 5, 20

operation preserving a relation, 6

P-compatible, 88

perspective intervals, 22

polynomially isomorphic sets, 21

prime interval, 11

projections, 8,19

pseudo-meet operation, 23

Q-compatible, 84

quasi-2-decomposability, 30

quasi-centralizer, 47

quasi-majority function, 31

reduct, 24

relation, 8,19

restriction of CSP, 8, 20 
retraction, 66

s-path, 25

semilattice free algebra, 14, 62

semilattice path, 25

semilattice type, 23

semilattice-connected component, 25

separated intervals, 11, 35

separating polynomial, 11,35

sm-smooth algebra, 24

strongly chained relation, 57

strongly semilattice-connected component, 25

subdirect product, 8,19

subdirectly irreducible algebra, 13

subdirectly irreducible instance, 61

subtrace, 22, 34

subtrace connected, 36

T-compatible, 91

tail, 22

term operation, 7

thin affine edge, 25

thin majority edge, 25

thin semilattice edge, 25

tightening, 9

tightening of a CSP, 20

trace, 22

type function, 20

u-maximal, 27

universal algebra, 7

universe, 7

witness of a type, 23 
Notation

$[n], 19$

$\leq, 25$

$\sqsubseteq, 25$

巨as, 27

$\sqsubseteq$ asm, 27

$\langle\mathbf{s}, R\rangle, 20$

$\underline{0}_{\mathbb{A}}, \underline{1}_{\mathbb{A}}, 19$

$\bar{\alpha}, 19$

$\bar{\alpha}_{I}, 19$

$\zeta(\alpha, \beta), 46$

$\tau_{U}, 70$

$\tau_{C}, 71$

$\tau_{C^{\prime}}^{\prime}(u, \eta \theta), 78$

$\mathbf{a}[i], 19$

$\bar{A}, 19$

$\bar{A}_{I}, 19$

$\operatorname{amax}(\mathbb{A}), 27$

as $(a), 27$

Center $(\mathcal{P}), 63$

$\mathrm{Cg}(\cdot), 19$

$f^{\mathbf{a}}(x), 46$

$\mathrm{Ft}_{\mathbb{A}}(a), 28$

$\mathrm{Ft}_{\mathbb{A}}^{a s}(a), 28$

$\mathrm{Ft}_{\mathrm{A}}^{a s m}(a), 28$

$\mathcal{G}(\mathbb{A}), 23$

$h_{a b}, 26$

$\mathrm{Ik}_{1}, \mathrm{Ik}_{2}, 31$

maj, 30

$\max (\mathbb{A}), 25$

$\operatorname{MAX}(\mathcal{P}), 62$

$p(\mathcal{P}), 66$

$\mathcal{P}=(V, \mathcal{C}), 20$

$\mathcal{S}_{\mathcal{P}}, 20$

$\mathcal{P}_{W}, 20$

$\mathcal{P}_{W^{\prime}}^{\prime}, 76$

$\mathcal{P}_{(v, \mathbb{B})}, 20$

$\mathcal{P}_{C \rightarrow C^{\prime}}, 20$

$\mathcal{P} / \bar{\alpha}, 20$
$\mathcal{P}^{\mathcal{R}}, 67$

$P^{x}, P^{x y}, 88$

$\operatorname{Pol}(\mathbb{A}), \operatorname{Pol}_{1}(\mathbb{A}), 19$

$\operatorname{pr}_{I} R, 19$

$Q^{x}, Q^{x y}, 84$

$Q_{a b}^{\mathbb{A}}, 33$

$R^{X}, 21$

$\mathcal{R}, 21$

$R[c], R^{-1}\left[c^{\prime}\right], 31$

$\mathcal{S}_{\mathcal{P}}, 20$

$\mathcal{S}_{W}, 20$

$\mathrm{Sg}(B), 19$

$\mathcal{S}^{\mathcal{R}}, \mathcal{S}^{\mathcal{R}^{\prime}}, 67$

$\mathcal{S}_{W^{\prime}}^{\prime}, 76$

$\operatorname{size}(\mathcal{P}), 62$

$t_{a b}, 26$

tol $_{1}$, tol $_{2}, 31$

$T^{x}, T^{x y}$,91

$\operatorname{umax}(\mathbb{A}), 27$

$\mathcal{W}^{\mathcal{P}}(\bar{\beta}), 51$

$W_{v, \alpha \beta, \bar{\beta}}, 51$

$\mathcal{W}^{\prime}(\bar{\beta}), 62$

$\mathrm{Z}_{\mathbb{A}}$,49 